Published in "Advances in atomic, molecular, and optical physics

49: 85-216, 2003"

which should be cited to refer to this work.

\title{
RESONANCE AND THRESHOLD PHENOMENA IN LOW-ENERGY ELECTRON COLLISIONS WITH MOLECULES AND CLUSTERS
}

\author{
H. HOTOP ${ }^{1}$, M.-W. RUF ${ }^{1}$, M. ALLAN $N^{2}$ and I. I. FABRIKANT \\ ${ }^{1}$ Fachbereich Physik, Universität Kaiserslautern, 67653 Kaiserslautern, Germany \\ ${ }^{2}$ Département de Chimie, Université de Fribourg, 1700 Fribourg, Switzerland \\ ${ }^{3}$ Department of Physics and Astronomy, University of Nebraska, Lincoln, NE, USA
}

\section{Introduction}

\section{A. Setting the Scene}

Low-energy collisions of electrons with atoms and molecules are among the most important elementary processes in gaseous environments such as

*E-mail: hotop@physik.uni-kl.de 
discharges, arcs, gas lasers, gaseous dielectrics and the earth's atmosphere. Correspondingly these processes have been studied for a long time, most notably over the last forty years following the improvement of instrumental technology and the detection of prominent resonance structure in electron scattering cross-sections due to the formation of temporary negative ions (TNI) (Schulz, 1973a, b). A wealth of information has been gained on the dynamics of these collisions, as documented by many reviews and books (e.g. Schulz, 1973a, b; Lane, 1980; Trajmar et al., 1983; Christophorou, 1984; Shimamura and Takayanagi, 1984; Allan, 1989; Domcke, 1991; Buckman and Clark, 1994; Crompton, 1994; Filippelli et al., 1994; Trajmar et al., 1994; Huo and Gianturco, 1995; Andersen and Bartschat, 1996; Chutjian et al., 1996; Zecca et al., 1996; Becker et al., 2000; Illenberger, 2000; Winstead and McKoy, 2000; Christophorou and Olthoff, 2001a, b; Karwacz et al., 2001a, b; Brunger and Buckman, 2002). In spite of this progress, however, the exploration of threshold phenomena in electronmolecule collisions at low energies - both in the limit of zero electron energy and in the neighborhood of onsets for vibrational excitation - has remained a major challenge for experiment and theory. Experimentally, it is difficult to achieve the desired resolution (energy width in the $\mathrm{meV}$ range) and to handle electron beams at energies below about $0.1 \mathrm{eV}$. Theoretically, it is demanding to incorporate the nuclear dynamics, using descriptions which go beyond local complex potential models (Burke, 1979; Lane, 1980; Kazansky and Fabrikant, 1984; Morrison, 1988; Domcke, 1991; Huo and Gianturco, 1995; Winstead and McKoy, 2000). In this article, we shall survey some of the insight gained over the past ten years through experimental investigations and theoretical descriptions of resonance and threshold phenomena occurring in low-energy electron collisions with molecules and molecular clusters. We concentrate on work carried out with very high resolution (energy width $1-10 \mathrm{meV}$ ) and electron energies typically below $1 \mathrm{eV}$. Much of that work has been devoted to anion formation through electron attachment, but we shall also present examples for total, elastic and vibrationally inelastic electron scattering. Electron impact induced neutral dissociation, electronic excitation, and ionization processes are not considered in this article. We mention, however, recent intriguing observations on vibrational resonances in positron-annihilation collisions with molecules. In the remaining part of Section I, we present a brief qualitative introduction into the field of low-energy electron-molecule collisions. Recent complementary surveys on electron collisions with molecules and clusters include several articles in journals (Hashemi et al., 1990; Domcke, 1991; Märk, 1991; Illenberger, 1992; Smith and Španel, 1994; Dunning, 1995; Chutjian et al., 1996; Ingolfsson et al., 1996; Zecca et al., 1996; Burrow et al., 1997; Field et al., 2001a; Karwacz et al., 
2001a, b; Brunger and Buckman, 2002) and book chapters (Trajmar et al., 1994; Huo and Gianturco, 1995; Becker et al., 2000; Illenberger, 2000; Winstead and McKoy, 2000; Christophorou and Olthoff, 2001a,b; Hotop, 2001; Tanaka and Sueoka, 2001).

\section{B. Dynamics of Low-Energy Electron-Molecule Collisions}

The dynamical behaviour of slow electrons traversing gases is to a large extent determined by two effects: the energy dependent evolution of the scattering phases for the relevant partial waves and the influence of temporary negative ion states (resonances). For quite a few atoms and molecules, special behaviour of the s-wave $(l=0)$ phase shift leads to a deep Ramsauer-Townsend minimum in the scattering cross-section between 0 and $1 \mathrm{eV}$ which strongly affects the electron mobility in these gases. Even more importantly, resonances (compound states of the electron-molecule system with lifetimes ranging typically from $10^{-15}$ to $10^{-11} \mathrm{~s}$ ) are often found to dominate the dynamics of electron-molecule collisions over the energy range $0-10 \mathrm{eV}$. The extended time interval (compared with the direct transit time which is below $1 \mathrm{fs}$ ), spent by the incoming electron close to the target while in the resonance state (lifetime $\tau=\eta / \Gamma, \Gamma=$ resonance width), has profound effects especially on collision channels which involve a reaction of the nuclear framework, i.e. on vibrational excitation VE and on dissociative attachment DA (forming negative ions). Apart from wellknown shape resonances such as $\mathrm{H}_{2}^{-}\left({ }^{2} \Sigma_{\mathrm{u}}\right), \mathrm{N}_{2}^{-}\left({ }^{2} \Pi_{\mathrm{g}}\right), \mathrm{CO}^{-}\left({ }^{2} \Pi\right), \mathrm{O}_{2}^{-}\left({ }^{2} \Pi_{\mathrm{g}}, v \geq 4\right)$ (Schulz 1973b; Shimamura and Takayanagi, 1984; Allan, 1989; Domcke, 1991; Brunger and Buckman, 2002) which are located below the lowest limit for DA and owe their lifetime to the centrifugal barrier of the electron, repulsive anion states above the DA limit are important for VE as well as DA. The importance of resonances for vibrational excitation (VE) as well as negative ion formation via dissociative attachment (DA) is illustrated in Fig. 1.

A resonance is formed when the incoming electron, possessing an energy $E$ close to the resonance energy, is captured into a low-lying unoccupied molecular orbital (LUMO) which typically has anti-bonding character. During the lifetime of the resonance the nuclei start to move to larger distances under the influence of the destabilizing force brought into the system by the captured electron. When the electron leaves the negative ion complex by autodetachment after a time comparable to $\tau$, the nuclei find themselves at a distance substantially larger than the equilibrium distance of the neutral molecule, i.e. in a vibrationally excited state. If the lifetime is sufficiently long to allow propagation of the nuclei 


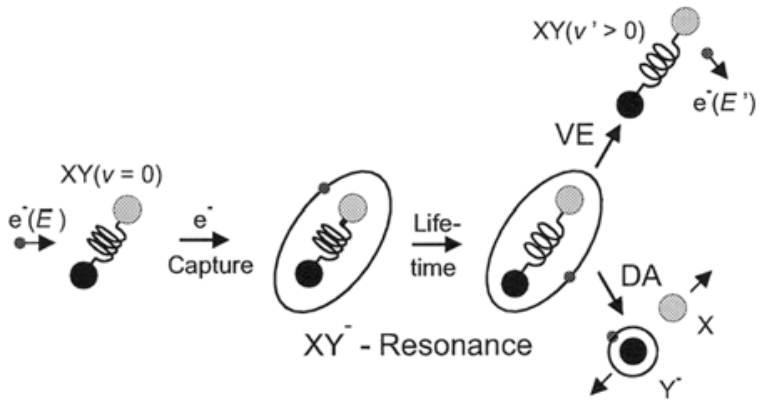

FIG. 1. Dynamics of vibrational excitation (VE) and dissociative attachment (DA) in electron-molecule scattering through resonances (from Hotop, 2001).

to large distances, dissociative attachment (DA, i.e. formation of $X+Y^{-}$ or $X^{-}+Y$ ) occurs. The occurrence of VE and DA is thus mediated by electron scattering through resonances $X Y^{-*}$, as summarized in the reaction scheme (1):

$$
\begin{aligned}
e^{-}(E)+\mathrm{XY}(n, v, J) & \rightarrow X Y^{-*}(\text { Resonance) } \\
& \rightarrow X Y\left(n^{\prime}, v^{\prime}, J^{\prime}\right)+\mathrm{e}^{-}\left(E^{\prime}\right) \quad \text { (Scattering) } \\
& \rightarrow X+Y^{-} \quad \text { (Dissociative Attachment) } \\
& \rightarrow X Y^{-} \quad \text { (Non-dissociative Attachment) }
\end{aligned}
$$

Process (1a) describes elastic scattering $\left(E=E^{\prime}\right)$ when the electronic $(n)$, vibrational $(v)$ and rotational $(J)$ quantum numbers all remain the same. A reaction with $v^{\prime}>v$ and $n=n^{\prime}$ corresponds to VE within the initial electronic state. Process (1c) describes nondissociative attachment (NDA), i.e. formation of negative ions $X Y^{-}$with lifetimes sufficiently long to allow mass spectrometric detection. Figure 2 illustrates the dynamics of vibrational excitation (VE), dissociative attachment (DA) and nondissociative attachment (NDA) in a potential curve diagram responsible for the nuclear motion along the normal coordinate $R$.

For the situation described by the potential curves in Fig. 2, VE and DA proceed through electron capture from the neutral ground state potential $V_{0}(R)$ into the repulsive TNI state $X Y^{-*}$ (potential $V_{-}{ }^{*}(R)$ ) which possesses a resonance width $\Gamma(R)$ accounting for autodetachment of the TNI at internuclear separations smaller than the crossing radius $R_{C}$; the width normally rises with decreasing $R$, but may saturate towards smaller $R$. The shaded area represents the Franck-Condon region for the primary electron capture process involving a molecule $X Y$ in its vibrational ground 


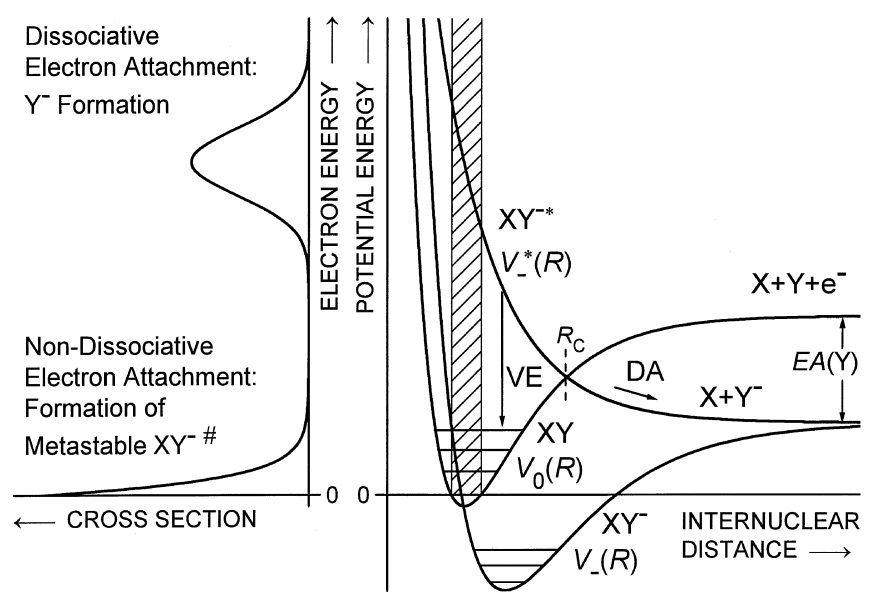

FIG. 2. Potential curve diagram for low-energy electron-molecule collisions.

state $v=0$. The energy dependences of the cross-section $\sigma_{v v^{\prime}}(E)$ for $\mathrm{VE}$ and $\sigma_{\mathrm{DA}}(E)$ for DA (which are similar, but not identical in shape (O'Malley, 1966, 1967; Chu and Burrow, 1994) reflect the Franck-Condon overlap of the initial $v=0$ level with the nuclear wave function in the $X Y^{-*}$ resonance state in combination with the effects of autodetachment. For vibrationally excited states in $V_{0}(R)$, the effective threshold energy for DA moves to lower energies, and a substantial effect of initial vibrational excitation is thus expected on the shape of the attachment spectrum (O’Malley, 1967; Massey, 1976).

At energies close to $0 \mathrm{eV}$, electron capture occurs into the low-lying anion state $X Y^{-}$(potential $V_{-}(R)$ ) with favorable Franck-Condon factors. In the depicted case, dissociation out of this anion state is not accessible at low electron energies. For small molecules (e.g. $\left.\mathrm{O}_{2}^{-}\left({ }^{2} \Pi_{\mathrm{g}}, v \geq 4\right)\right)$, autodetachment occurs within the characteristic lifetime of the resonance, unless the TNI is stabilized by vibrational deexcitation in collisions with other particles (as possible in high density media or in clusters). For molecules with sufficient complexity (such as $\mathrm{SF}_{6}, \mathrm{C}_{6} \mathrm{~F}_{6}$ or $\mathrm{C}_{60}$ ), however, the total energy of the $X Y^{-}$ system may be distributed efficiently over the rich vibrational manifold by intramolecular vibrational redistribution (IVR) in such a way that it can take a long time before the system finds itself again in a situation favourable for autodetachment, i.e. nondissociative attachment with formation of a metastable anion occurs. The effective lifetime of the $X Y^{-}$anion in general depends on the initial vibrational energy. The energy dependence of the cross-section for NDA typically peaks at zero energy and decreases rapidly with rising energy (Christophorou, 1978; Illenberger, 2000). 
There are quite a number of important cases (e.g. $\mathrm{F}_{2}, \mathrm{Cl}_{2}, \mathrm{O}_{3}, \mathrm{CCl}_{4}$, $\mathrm{CFCl}_{3}$ ) for which the repulsive branch of the lowest potential surface for the $X Y^{-}$anion cuts the neutral ground state potential near the minimum of the latter (as in Fig. 2), but has its lowest asymptotic limit $X+Y^{-}$lying energetically below the vibrational ground state of $X Y$. In this case, DA is exothermic at zero electron energy, and one expects to observe a zero energy peak in the DA cross-section due to s-wave attachment unless the $l=0$ partial wave is forbidden by symmetry considerations. Like neutron capture by nuclei at low energies, the cross-section for s-wave electron attachment to molecules follows the $1 / v$ law (Bethe, 1935; Wigner, 1948) when the electron velocity $v$ goes to zero. While beautiful confirmations of this law have been made in neutron absorption experiments some time ago (e.g. Blatt and Weisskopf, 1952), clear demonstrations for electron capture collisions had to wait until sub-meV resolution was achieved in laser photoelectron attachment experiments (Klar et al., 1992a, b; Schramm et al., 1998).

Interesting resonance and threshold phenomena may occur close to onsets for vibrational excitation: the channels of the electron-molecule scattering system $(1 \mathrm{a}, \mathrm{b}, \mathrm{c})$ are coupled and thus 'feel' each other. This channel coupling leads to special structure (Wigner cusps of different shapes, vibrational Feshbach resonances) in open channels when the collision energy $E$ passes through the onset for a new inelastic channel. High energy resolution is needed to reveal these features. For a variety of molecules, threshold peaks, i.e. large enhancements in VE cross-sections within a narrow region above the VE onset, are observed, as first discovered for $\mathrm{HF}$ and $\mathrm{HCl}$ by Rohr and Linder (1975, 1976). Towards higher energies, VE cross-sections may exhibit oscillatory 'boomerang' structures (Birtwistle and Herzenberg, 1971; Herzenberg, 1984), found more recently even in molecules like $\mathrm{HCl}$ where they would initially not be expected because of a large resonance width (Cvejanovic, 1993; Allan et al., 2000; Ćížek et al., 2001). Very narrow 'outer well resonances', superimposed on the broader boomerang structures, have been identified, initially theoretically, in molecules where the potential curve of the anion has a secondary minimum at large internuclear separation (Allan et al., 2000; Čizek et al., 2001). Remarkable progress towards a deeper understanding of these phenomena has been recently achieved in a systematic joint experimental and theoretical investigation of the hydrogen halides (see Section IV.).

For molecules with sufficiently strong long-range electron-molecule interactions (e.g. due to the molecular polarizability or dipole moment) the existence of a quasi-discrete low-energy anion state in the continuum $\left(V_{\mathrm{d}}(R)\right.$, electron attached to the LUMO) leads to two potential curves $V_{\text {res, } 1}(R)$ and $V_{\text {res,2 }}(R)$ (Domcke and Cederbaum, 1981; Gauyacq and Herzenberg, 1982), as sketched in Fig. 3b. Note that without the long-range 



FIG. 3. On the origin of vibrational Feshbach resonances: sketch of potentials without (a) and with (b) long-range electron-molecule interaction.

attraction, only one resonance $V_{\text {res }}(R)$ exists (somewhat shifted below $V_{\mathrm{d}}(R)$ by the interaction of $V_{\mathrm{d}}$ with the continuum), as shown in Fig. 3a.

For internuclear distances around the equilibrium separation $R_{\mathrm{e}}$ of the neutral curve $V_{0}(R)$, the potential curve $V_{\text {res, } 2}(R)$ lies close to, but below $V_{0}(R)$; in this range, the anion state $V_{\text {res, } 2}(R)$ may be viewed as a composite of the neutral molecule with a diffuse electron bound by long-range forces (with no need to invoke a centrifugal barrier for the electron). As evident from Fig. 3b, the low-lying vibrational levels $v_{2}$ in $V_{\text {res, } 2}(R)$ are located just below the corresponding vibrational levels $v_{0}=v_{2}$ in the neutral molecule. The level $v_{2}=0$ may be - when sufficiently bound with respect to the influence of rotational effects - a stable, detectable anion state (such as a dipole-bound state, Desfrançois et al., 1996) which is not accessible in collisions of the molecule with free continuum electrons. The quasi-bound vibrational levels in $V_{\text {res,2 }}(R)$ with $v_{2} \geq 1$ lie in the electron-molecule continuum and correspond to vibrational Feshbach resonances (VFR, Schramm et al., 1999); they were previously addressed as nuclear-excited Feshbach resonances (Bardsley and Mandl, 1968; Gauyacq and Herzenberg, 1982; Knoth et al., 1989a; Thümmel et al., 1993). In the depicted case, the VFRs can only decay by autodetachment through indirect coupling to the continuum (via kinetic coupling of the two resonance states). The VFRs are thus expected to live quite long and to appear as narrow features below vibrational thresholds in elastic or vibrationally inelastic electron scattering cross-sections (Knoth et al., 1989a; Thümmel et al., 1993; Schramm et al., 
1999; Sergenton et al., 2000); their observation requires high energy resolution. If the asympotic energy of the discrete anion state $V_{\mathrm{d}}(R)$ lies below a given VFR, then this and higher-lying VFRs may also decay by dissociation and thus be observed in DA as well (Schramm et al., 1999, 2002). Interestingly, vibrational resonances have been recently also observed in the energy dependent cross-sections for positron annihilation involving polyatomic molecules in the gas phase (Gilbert et al., 2002), see Section IV.D.

The following article is organized as follows. In Section II, we survey the relevant aspects of the underlying theory. In Section III, we describe experimental aspects with emphasis on recent developments. In Section IV, selected case studies highlight some of the recent progress in the field. In Section V, we conclude with a brief summary and address some unsolved problems.

\section{Theory}

Theoretical developments in the field of electron-molecule collisions during the past twenty-five years were covered in several reviews (Burke, 1979; Lane, 1980; Herzenberg, 1984; Kazansky and Fabrikant, 1984; Morrison, 1988; Domcke, 1991; Huo and Gianturco, 1995). Some aspects of resonance and threshold phenomena were discussed in these reviews, as well as by Sadeghpour et al. (2000). The theoretical description of electron-molecule collisions generally requires an adequate description of electronic, vibrational and rotational degrees of freedom. However, if the typical collision time is short compared to the rotational period, the molecule can be treated as having a fixed orientation during the collision process, and the result for the cross-section can be averaged over orientations. Treatment of vibrational dynamics is usually more important and more challenging to the theory. In the electron energy region important for applications, many inelastic processes such as vibrational excitation and dissociative electron attachment are driven by negative-ion resonances, as already addressed in the introduction. The lifetime of these resonance states is quite often comparable to the vibrational period (e.g., for $\mathrm{N}_{2}, \mathrm{CO}$ and $\mathrm{CO}_{2}$ molecules) and sometimes even exceeds it substantially (e.g., for the $\mathrm{O}_{2}$ molecule). The theoretical description of vibrational dynamics in these cases is usually based on the nonlocal complex potential describing the nuclear motion in the intermediate negative-ion state (O'Malley, 1966; Bardsley, 1968; Domcke, 1991). An alternative method is based on the $R$-matrix approach (Schneider et al., 1979; Fabrikant, 1990). For studies of resonance and threshold phenomena the latter one is especially attractive, particularly in the case 
of a strong long-range interaction between the incident electron and the molecular target. The $R$ matrix allows a convenient parametrization, and all strong energy dependences of transition amplitudes and cross-sections can be accounted for by inclusion of the long-range interaction in electronic channels. In what follows we will briefly describe this approach formulated by Wigner (1948) and its application to electron-molecule collisions.

\section{A. Multichannel $R$-Matrix Theory}

The fundamental paper of Wigner (1948) gives a unified method for the description of inelastic processes in the near-threshold region. The method is based on the $R$-matrix theory (Wigner and Eisenbud, 1947) which was initially developed for nuclear reactions, but later on applied to electron-atom and electron-molecule collisions. The $R$-matrix theory is a very powerful tool for analytical studies of near-threshold and resonance phenomena, as well as for ab initio numerical calculations.

The basic concept of Wigner's theory is the reaction sphere outside which only long-range interactions between reactants are important. Initially it was assumed that this interaction is diagonal, that is interchannel transitions are impossible outside the sphere. This assumption is not always valid in the theory of electron-molecule collisions. In particular, the dipolar interaction can cause transitions between different rotational states. The $R$-matrix theory can be generalized for a nondiagonal dipolar interaction outside the sphere (Gailitis and Damburg, 1963). However, most of the analytical results (with the exception of dipolar interaction between degenerate channels) were obtained assuming a diagonal long-range interaction, and we will use this approximation at the first stage.

\section{A.1. Analytical Theory of Threshold Behavior, Resonances, and Cusps: Short-Range Interaction}

We consider an $N$-channel system and introduce the multichannel wave function in the form of an $N \times N$ matrix $\psi$ which has the following form outside the reaction sphere, $r>r_{0}$,

$$
\boldsymbol{\psi}=\boldsymbol{h}^{-1}-\boldsymbol{h}^{(+)} \boldsymbol{S}
$$

where $\boldsymbol{S}$ is the scattering matrix and $\boldsymbol{h}^{( \pm)}$are channel wavefunctions with the following asymptotic behavior

$$
h_{i j}^{ \pm} \sim \delta_{i j} k_{i}^{-1 / 2} \exp \left[i\left(k_{i} r-l_{i} \pi / 2\right)\right],
$$


where $k_{i}$ and $l_{i}$ are linear and angular momenta in channel $i$. The asymptotic form (3) assumes that all $N$ channels are open. However, the whole treatment can be easily generalized if there are $N_{\mathrm{c}}$ additional closed channels. The corresponding diagonal matrix elements of $\boldsymbol{h}^{(+)}$behave like $\exp -\mathrm{i}\left|\mathrm{k}_{\mathrm{i}}\right| \mathrm{r}: \exp \left(-\mathrm{i}\left|\mathrm{k}_{\mathrm{i}}\right| \mathrm{r}\right)$, and the matrice $\boldsymbol{h}^{(-)}$and $\boldsymbol{S}$ become rectangular with $N+N_{\mathrm{c}}$ rows and $N$ columns. For simplicity we will not dwell on further details related to closed channels.

The partial (for a given set of angular momenta) cross-section for transitions from an initial state $i$ to a final state $f$ is proportional to $\left|T_{f i}\right|^{2}$ where $\boldsymbol{T}$ is the transition matrix related to the scattering matrix by

$$
\boldsymbol{T}=\mathbf{1} \text { (= unity matrix) }-\boldsymbol{S}
$$

The function (2) is matched with the internal wavefunction in the form

$$
\psi=R \frac{\mathrm{d} \psi}{\mathrm{d} r}
$$

where $\boldsymbol{R}$ is the Wigner $R$ matrix which is a meromorphic function of energy having poles only on the real axis (Lane and Thomas, 1958). For the purpose of derivation of the threshold laws we assume that the $R$ matrix can be considered as an analytical function of energy and expanded in powers of energy $E$. This is the essence of the effective range theory (ERT). A special treatment is necessary if there is a bound, a virtual, or a resonance state near the threshold.

Using analytical properties of $\boldsymbol{h}^{(+)}$, one can obtain the equation of Ross and Shaw (1961)

$$
\boldsymbol{T}=-2 i k^{l+1 / 2}\left(\boldsymbol{M}-i k^{2 l+l}\right)^{-1} k^{l+1 / 2},
$$

where $\boldsymbol{M}$ is a meromorphic function of energy. This equation allows us to obtain threshold laws for elastic and inelastic processes. In particular the threshold law for an inelastic process is given by

$$
T_{f i} \sim k_{f}^{l_{f}+1 / 2} .
$$

This is the Wigner threshold law. Usually we are interested in the total (that is, summed over all orbital angular momenta) cross-section. Then $l_{f}$ is the lowest angular momentum allowed by the symmetry of the problem. For example, photodetachment of a bound s electron gives a $k_{f}^{3}$ or $E_{f}^{3 / 2}$ behavior of the cross-section ( $\mathrm{p}$-wave emission), whereas photodetachment 
of a bound $\mathrm{p}$ electron leads to the $k_{f}$ behavior (s-wave emission) near threshold because of the dipole selection rules (the d-wave is suppressed strongly by the $k_{f}^{5}$ dependence).

In nonresonant collision processes, when there is no selection rule in $l$, the lowest allowed angular momentum is $l_{f}=0$, therefore we obtain $\sigma_{f i} \propto k_{f}$ for the cross-section of an endothermic process. This law can be usually applied to the process of electron impact excitation of atoms and molecules, if there is no dipolar interaction in the final channel. If heavy particles are formed in the final state (for example in DA and electron impact dissociation processes) the range of validity of the Wigner law is very narrow (Fabrikant et al., 1991), therefore, if there is no activation barrier for the process, the cross-section as a function of energy in the near-threshold region exhibits a vertical onset (O'Malley, 1966).

Another example is the Wigner-Baz' cusp (Baz', 1958). Just for illustration we will consider now a two-channel case and investigate the behavior of $\left|T_{11}\right|^{2}$ near the threshold for excitation of the channel 2. Note that $k_{2}$ is real above the threshold, and purely imaginary below the threshold. This allows us to write $\left|T_{11}\right|^{2}$ in the following form

$$
\begin{gathered}
\left|T_{11}\right|^{2}=\frac{a}{b+c k_{2}^{2 l_{2}+1}}, \quad k_{2}^{2}>0 \\
\left|T_{11}\right|^{2}=\frac{a+d\left|k_{2}\right|^{2 l_{2}+1}}{b+f\left|k_{2}\right|^{2 l_{2}+1}}, \quad k_{2}^{2}<0
\end{gathered}
$$

where the constants $a, b, c, d$, and $f$ are expressed through the elements of the $M$-matrix. Equation (9) explicitly demonstrates the discontinuity of $\mathrm{d}\left|T_{11}\right|^{2} / \mathrm{d} E$ at threshold (the threshold cusp) for $l_{2}=0$. If $l_{2}>0$, we still have formally a nonanalytical behavior appearing as a discontinuity of higher derivatives. However, this behavior is very hard to detect experimentally. The paper of Baz' (1958) shows that this discontinuity results directly from the conservation of probability or the unitarity of the $S$ matrix.

Note that the explicit expressions for the coefficients allow us to prove (Fabrikant, unpubl.) that $a, b$, and $c$ are positive meaning that the elastic cross-section is always decreasing above the threshold. At the same time there is no certain relation between $d$ and $f$, therefore the sign of the energy derivative below the threshold might be both positive and negative. This result can be generalized to some multichannel cases. For example, if there are two open channels and we look at the transition $1 \rightarrow 2$, it can be shown that in the vicinity of the threshold for channel 3 the cross-section $\sigma_{12}$ exhibits the same type of behavior: the sign of $\mathrm{d} \sigma_{12} / \mathrm{d} E$ might be both 


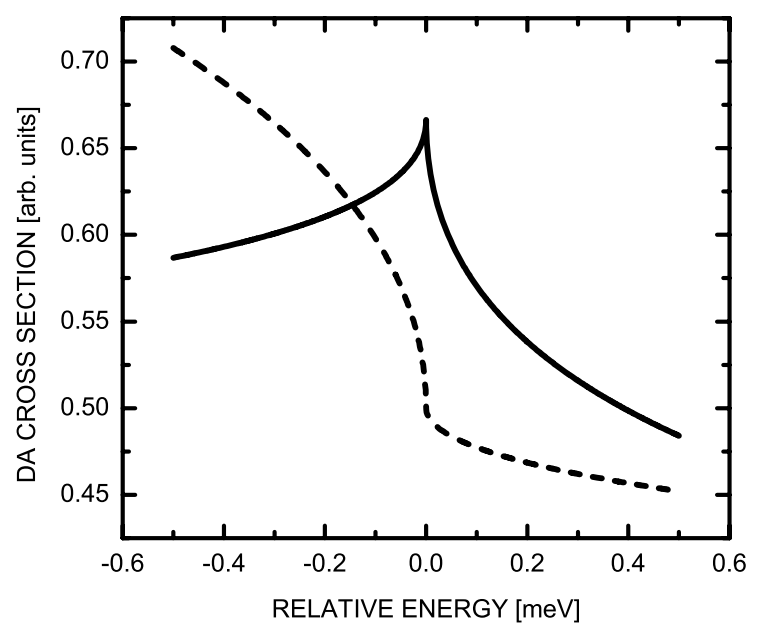

FIG. 4. Illustration of cusp structure in partial cross-sections due to interchannel coupling (see text).

positive and negative below threshold, but is always negative above threshold. This behaviour is illustrated in Fig. 4 and can be found, e.g., in cross-sections for dissociative attachment around a vibrational excitation threshold. Indeed, in all observed and calculated cases which involve long-range interactions decaying faster than $r^{-2}$ the DA cusp goes downward above the VE threshold.

Although these results seem to be natural (a cross-section in the 'old' channel is decreasing because of the growing cross-section for a transition into a 'new' channel), they are not generally true. In particular, they do not apply when there is a long-range (dipolar) interaction outside the $R$-matrix sphere. The case of the Coulomb interaction gives a completely different behavior, but we do not discuss it here since this review is concerned with electron scattering by neutral targets.

Equation (6) contains also information about near-threshold resonances and virtual states. Let us again consider for simplicity a two-channel case with $l_{2}=0$. Assume first that interchannel coupling is negligible, that is $M_{12}=0$. Then the $T$-matrix has a pole in the complex $k_{2}$ plane whose position is $k_{2}=-i M_{22}$. This corresponds to a bound state when $M_{22}<0$ and a virtual state when $M_{22}>0$. If $M_{12} \neq 0, k_{2}$ acquires a nonzero real part. When $M_{22}<0$ it corresponds to a Feshbach resonance whereas the case $M_{22}>0$ corresponds to a virtual state coupled to channel 1 . In the first case there is a time delay in scattering, and the cross-section exhibits a Lorentzian or a Fano profile, whereas in the second case the cross-section 
exhibits an enhancement at the threshold. This phenomenon is closely connected to the threshold cusps discussed above. In the case of a virtual state whose corresponding pole is close to $k_{2}=0$ the cross-section exhibits a very sharp cusp with a positive derivative at $k_{2}^{2}<0$ and a negative derivative at $k_{2}^{2}>0$. If the interaction in the channel 2 becomes more attractive, the cusp turns into a Feshbach resonance below the threshold. Examples illustrating this behavior will be given in Section II.A.4.

\section{A.2. Dipolar Interaction: Stationary Dipole}

A similar approach can be applied in the case of a long-range interaction outside the reaction sphere. In case of electron interaction with a nonrotating dipolar molecule the problem is reduced to diagonalization of the operator (Mittleman and von Holdt, 1965)

$$
\boldsymbol{L}=\boldsymbol{l}(\boldsymbol{l}+1)-2 \boldsymbol{D},
$$

where $\boldsymbol{D}$ is the dipole moment matrix obtained by calculating the matrix element of the dipolar interaction between the angular momentum eigenstates.

Diagonalization of the matrix $\boldsymbol{L}$ allows us to express the solution of the Schrödinger equation outside the reaction sphere as a linear combination of the Bessel functions with indices $\lambda_{i}+1 / 2$ where $\lambda_{i}$ are related to the eigenvalues $\Lambda_{i}$ of the matrix $\boldsymbol{L}$ by

$$
\Lambda_{i}=\lambda_{i}\left(\lambda_{i}+1\right), \quad i=0,1, \ldots
$$

The form of the threshold law critically depends on the lowest eigenvalue $\Lambda_{0}$ (Gailitis and Damburg, 1963). If $\Lambda_{0}>-1 / 4$, all $\lambda_{i}$ are real, the $T$-matrix element for inelastic processes is proportional to $k^{\lambda_{0}+1 / 2}$, and the crosssection to $k^{2 \lambda_{0}+1}$. This happens if the dipole moment of the molecule is lower than the critical dipole moment $\mu_{\mathrm{cr}}=0.6395$ a.u. $=1.625 \mathrm{D}$. If $\mu>\mu_{\mathrm{cr}}$, or $\Lambda_{0}<-1 / 4, \lambda_{0}+1 / 2$ is purely imaginary, and the cross-section is finite at the threshold. The cross-section for an inelastic process can be written as (Fabrikant, 1977,1978) $\left(k \equiv k_{f}\right)$.

$$
\sigma_{f i}=\text { const }\left|\mathrm{e}^{i \beta}+\mathrm{e}^{\pi \tau} k^{2 i \tau}\right|^{-2},
$$

where $\tau=\operatorname{Im} \lambda$ and the parameter $\beta$ depends on elements of the $M$ matrix as well as on the dipole moment. Although $\beta$ is generally complex, its imaginary part is small if the interaction with other channels (other than dipole-coupled near-threshold channels) is weak. In particular $\beta$ is real for 
pure elastic scattering. In this case analytical continuation of the $T$ matrix below the threshold into the region of negative $k_{f}^{2}$ allows us to find the poles whose positions are given by the equation

$$
k^{2}=-\exp \left\{-[\pi(2 n+1)+\beta] \tau^{-1}\right\}
$$

These are the well-known dipole-supported states, discussed originally by Fermi and Teller (1947, see also Turner, 1977) and observed in a series of experiments on charge transfer from Rydberg atoms to polar molecules and clusters (Desfrançois et al., 1994a, b, c; Desfrançois et al., 1996; Compton and Hammer, 2001). Note that these states are very rapidly (exponentially) converging to the threshold, and this is what makes them very different from the Coulomb Rydberg states. The rotational splitting reduces the number of these states from infinity to very few, sometimes even to zero. For example, the HF molecule and water molecule have supercritical dipole moments, however they do not have stable anion states. Crawford and Garrett (1977), by performing model calculations for various molecules, concluded that a dipole-supported state remains bound after inclusion of rotation, if its fixed-nuclei binding energy exceeds approximately ten percent of the rotational constant.

If there are open channels below the threshold, the discussed bound states become dipole-supported Feshbach resonances. If the vibrational motion of the molecule is included, each dipole-supported state can generate a series of vibrational Feshbach resonances, originally called 'nuclear-excited' Feshbach resonances (Bardsley and Mandl, 1968; Domcke and Cederbaum, 1981; Gauyacq and Herzenberg, 1982).

Above the threshold the analytical structure of Eq. (12) leads to oscillations of the cross-section as a function of energy. However, these oscillations cannot be observed in practice (Fabrikant, 1977, 1978). If the dipole moment is just above the critical, the period of oscillations exceeds the rotational spacing whereas for higher dipole moments the amplitude of oscillations becomes exponentially small.

\section{A.3. Rotating Dipole}

Turning to the more complicated case of a rotating dipole, we have to distinguish between two cases. In the first, rotation removes all degeneracies of the dipole-coupled channels. In the second, some degenerate channels coupled by the dipolar interaction still remain. The second case is typical for symmetric-top molecules and molecules with nonzero projection of the electronic angular momentum on the internuclear axis (e.g., molecules in a $\Pi$ state). 
If the electron energy in the final state is small compared to the rotational spacing, the dipole coupling becomes equivalent to the action of a diagonal potential which behaves at large distances as a polarization potential $\alpha_{\text {eff }} /\left(2 r^{4}\right)$ (note that the effective polarizability $\alpha_{\text {eff }}$ can be both positive and negative). In the case of scattering by polar molecules the long-range behavior of the effective diagonal potential depends on the total angular momentum $J$. If $J=0$ the effective polarizability is given by (Clark, 1979; Fabrikant, 1983) $\alpha_{\mathrm{eff}}=\mu^{2} /(3 B)$ where $\mu$ is the permanent dipole moment and $B$ the rotational constant. For $J>0$ and s-wave electrons the effective (static) polarizability turns to zero, and the long-range behavior is determined by the dynamical polarization interaction decaying as $r^{-6}$. In all cases the Wigner threshold law is restored, and it is also possible to find an analytical correction to the Wigner law of the order of $\alpha_{\text {eff }} k_{f}^{2} \ln k_{f}$ (O'Malley, 1965; Damburg, 1968; Gailitis, 1970). The region of the transition between the Wigner law and the dipole threshold law is much more complicated. Even in the simplest two-channel case the solution has a very complicated analytical structure (Gailitis, 1970). Therefore most of the studies in this region were performed by numerical integration of the coupled equations (Fabrikant, 1978, 1983).

If the dipole moment of the molecule is supercritical, there is an infinite number of dipole-supported states in the fixed-nuclei approximation. When the rotational splitting is included, all or most of them disappear because of the effective cut-off of the dipole potential. At large distances the effective electron-dipole interaction decays as $r^{-4}$ or even faster. At shorter distances, where the rotational spacing is smaller than the electron-dipole interaction, the adiabatic body-frame representation (Clark, 1979; Fabrikant, 1983) is more appropriate for description of the physics. In this region the dipole potential leads to binding and anisotropy of the electron wavefunction. The size of the inner (adiabatic) region may be as large as a few hundred a.u. It means that the dipolar interaction may be strong enough to create a diffuse bound or a virtual state (Frey et al., 1994). In particular very diffuse virtual states were found in scattering of Rydberg electrons by HF molecules (Hill et al., 1996) and $\mathrm{CH}_{3} \mathrm{Cl}$ molecules (Frey et al., 1995; Fabrikant and Wilde, 1999).

In the presence of a bound or a virtual state near the threshold the analysis based on the multichannel formula of Ross and Shaw (1961), Eq. (6), leads to the following result for the transition cross-section $\left(k \equiv k_{f}\right)$

$$
\sigma_{f i}=\frac{a k}{k^{2}+2 k \operatorname{Im} \kappa+|\kappa|^{2}} .
$$


The parameters $\kappa$ and $a$, as well as the positions of the $S$-matrix poles depend on $J$. This dependence was calculated for HF (Fabrikant, 1996) molecules. For higher $J$ the pole is moving farther away from the origin, and its influence on the threshold behavior becomes weaker.

In symmetric-top molecules and diatomic molecules with nonzero $\Lambda$, rotation reduces the dipole moment, but does not average it to zero, as for diatomic molecules in a $\Sigma$ state and nonsymmetric polyatomic molecules. In diatomic molecules with $\Lambda \neq 0$ the channels with the projection of the electronic angular momentum $M= \pm \Lambda$ remain degenerate if $\Lambda$-doubling is neglected. For symmetric tops there is a degeneracy with respect to the sign of projection of the rotation angular momentum on the symmetry axis. The reduced dipole moment $\mu_{a v}$ can be defined as $\mu_{a v}=K /[J(J+1)]^{1 / 2}$, where $J$ is the total rotational angular momentum and $K$ its component about the symmetry axis. $K$-doubling and inversion splitting are neglected in this approximation. Detailed analyses of the threshold exponents for these cases was done by Engelking (1982) and Engelking and Herrick (1984). Application to the near-threshold photodetachment of $\mathrm{OH}^{-}$was presented by Smith et al. (1997). The position of the virtual-state poles as a function of $J$ and $K$ was calculated for the $\mathrm{CH}_{3} \mathrm{Cl}$ molecule by Fabrikant and Wilde (1999).

\section{A.4. Vibrational Dynamics}

At ultralow electron energies when the collision time is much longer than the vibrational period, the projectile electron "sees" the potential averaged over vibrations, therefore the theoretical description of vibrational motion is rather simple in this case. At electron energies which are substantially higher than the vibrational spacing, one can use the adiabatic approximation (Chase, 1956) whereby the transition amplitude is calculated by taking the matrix element of the fixed-nuclei amplitude between the initial and final vibrational states. The intermediate region, where the electron energy becomes comparable to the vibrational spacing, is the most challenging for theoretical calculations. On the other hand, due to the very large difference in masses of the projectile and the target, vibrational excitation of molecules by electrons and DA processes occur with substantial rates only when a resonance mechanism is involved whereby at first stage the electron is captured by the molecule forming a temporary negative-ion state. Inclusion of the resonance mechanism into the theory makes it simpler and more physically transparent, although alternative descriptions without the explicit use of the resonance states are possible (e.g. the zero-rangepotential (ZRP) description applied by Gauyacq, 1982). 
There are two methods for inclusion of the resonance mechanism into the theory of electron-molecule collisions: the Feshbach projection operator technique and the $R$-matrix approach. The first is more convenient for $a b$ initio calculations of VE and DA. However, because of substantial computational challenges, this approach has been applied so far only to diatomic molecules. Even a relatively simple triatomic molecule, $\mathrm{CO}_{2}$, has been treated (Kazansky, 1995; Rescigno et al., 2002) only in the so-called local approximation. The idea of the local approximation (O'Malley, 1966; Bardsley, 1968; Herzenberg, 1968), also called the boomerang model (Birtwistle and Herzenberg, 1971; Dubé and Herzenberg, 1979), is to describe the motion of the negative-ion state by the Schrödinger equation with a local complex potential whereas the actual potential describing this motion is a nonlocal energy-dependent operator (Domcke, 1991). The nonlocal effects become particularly important near vibrational excitation thresholds where the local theory fails to describe vibrational Feshbach resonances and threshold cusps.

The nonlocal effects can be successfully described within the framework of the projection-operator approach (Domcke, 1991; Meyer et al., 1991; Ćížek et al., 1999) or the resonance $R$-matrix theory. The ab initio $R$-matrix method (Schneider et al., 1979) requires several terms in the $R$-matrix expansion to describe a single resonance. This is not physically transparent and causes difficulties in the calculation of DA processes. In contrast, the effective $R$-matrix model (Wong and Light, 1984, 1986) and the resonance $R$-matrix model (Fabrikant, 1986) use only one $R$-matrix state corresponding to the physical resonance. This allows us to find the direct connection between the parameters of the $R$-matrix theory and parameters of the Feshbach projection operator approach, particularly the position and the width of the negative-ion resonance.

For model calculations we present the fixed-nuclei $R$ matrix in the form

$$
R=\frac{\gamma^{2}(\rho)}{W(\rho)-E_{\mathrm{e}}}+R_{\mathrm{b}},
$$

where the surface amplitude $\gamma(\rho)$ and the $R$-matrix pole $W(\rho)$ are standard parameters of the $R$-matrix theory, and $R_{\mathrm{b}}$ is a background term which is assumed to be weakly dependent on electron energy $E_{\mathrm{e}}$ and internuclear distance $\rho$ (here, $\rho$ is understood to represent its deviation from the equilibrium distance in the neutral potential energy curve). For simplicity we assume that only one angular mode dominates the resonance scattering, therefore the fixed-nuclei $R$ matrix includes only one channel. For example, the resonance scattering by the $\mathrm{N}_{2}$ molecule is dominated by the d-wave, 
whereas the resonant scattering by polar molecules is typically dominated by the lowest angular mode resulting from the diagonalization of the operator (10).

The nuclear motion is included by replacing function (15) by the operator (Schneider et al., 1979).

$$
R=\gamma(\rho)(T+U(\rho)-E)^{-1} \gamma(\rho)+R_{\mathrm{b}}
$$

where $T$ is the kinetic energy operator for the nuclear motion, $E$ is the total energy of the system (including the vibrational energy), and $U(\rho)=W(\rho)+V(\rho)$ is the potential energy of the negative-ion state (whereas $V(\rho)$ is the potential energy of the neutral molecule). Function $U(\rho)$ is equivalent to the diabatic negative-ion state of the projection-operator theory, although these two are not identical. In particular $U(\rho)$ depends on the $R$-matrix radius $r_{0}$. In model calculations we try to choose $r_{0}$ and other $R$-matrix parameters in such a way that one curve $U(\rho)$ represents the resonance which we want to describe.

The basic equation of the $R$-matrix theory (5) can then be formally solved. We introduce a diagonal matrix $\boldsymbol{u}^{+}$of radial electron wavefunctions in different vibrational channels and the surface amplitude matrix $\gamma$ for transitions between vibrational states of the neutral molecule and negativeion states. Then the $S$ matrix for DA can be written in the following form

$$
\boldsymbol{S}_{\mathrm{DA}}=2 \pi\left(\tilde{\boldsymbol{u}}^{+}\right)^{-1}\left(1+\gamma \mathbf{G}^{(+)} \gamma \mathbf{L}^{+}\right)^{-1} \boldsymbol{y},
$$

where $\tilde{\boldsymbol{u}}^{+}=\boldsymbol{u}^{+}-R_{\mathrm{b}}\left(\boldsymbol{u}^{+}\right)^{\prime}, \mathbf{L}^{+}=\left(\boldsymbol{u}^{+}\right)^{\prime}\left(\tilde{\boldsymbol{u}}^{+}\right)^{-1}, \mathbf{G}^{(+)}$is the Green operator for the nuclear motion in the negative-ion state, and $\mathbf{y}$ is the column of the firstorder DA amplitudes

$$
y_{v}=\left\langle v|\gamma| \psi^{(+)}\right\rangle
$$

where $|v\rangle$ is the eigenstate of the vibrational Hamiltonian for the neutral molecule, and $\psi^{(+)}$is the nuclear wavefunction describing the motion in the negative-ion state corresponding to the outgoing-wave boundary condition. Because of the importance of the vibrational continuum for the calculation of the DA processes, Eq. (17) is actually an integral equation for $\boldsymbol{S}_{D A}$. It is solved by the quasiclassical technique (Kalin and Kazansky, 1990) based on the quasiseparable representation of the Green operator.

The matrix $\mathbf{L}^{+}$in Eq. (17) is responsible for near-threshold resonances and cusps in partial cross-sections (including dissociative attachment) at 
each VE threshold. For example, in the absence of long-range electronmolecule interactions we obtain for the s-wave case

$$
L_{v}^{+}=\frac{i k_{v}}{1-i R_{b} k_{v}} .
$$

This behavior gives a cusp in $S_{\mathrm{DA}}$ at the threshold for vibrational excitation of the level $v$. However, the cusps are not very pronounced in the absence of long-range interactions. Much more pronounced cusps appear when we include polarization and/or dipolar interactions outside the $R$-matrix sphere. If this interaction is attractive enough, it leads to a vibrational Feshbach resonance below the threshold. Mathematically it corresponds to a pole of $S_{\mathrm{DA}}$, Eq. (17), in the complex plane of the energy $E_{v}=k_{v}^{2} / 2$ whose imaginary part is negative and gives the resonance halfwidth. If the imaginary part of the pole is positive, the pole corresponds to a virtual state shifted into the complex plane of $E_{v}$ because of the interchannel interaction. If the virtual state pole is close to $E_{v}=0$, we obtain a very sharp cusp at threshold. If we decrease the interaction in the channel $v$, the pole moves away from $E_{v}=0$, and the cusp becomes weaker. This situation is schematically represented in Fig. 5.

An alternative, and perhaps physically more transparent description of vibrational Feshbach resonances and threshold effects starts with the neutral curve $V(\rho)$ and the "diabatic" negative-ion curve $U(\rho)$. The adiabatic negative-ion curve can be obtained from the basic equation of the $R$-matrix theory, Eq. (5), but using now the fixed-nuclei approximation.

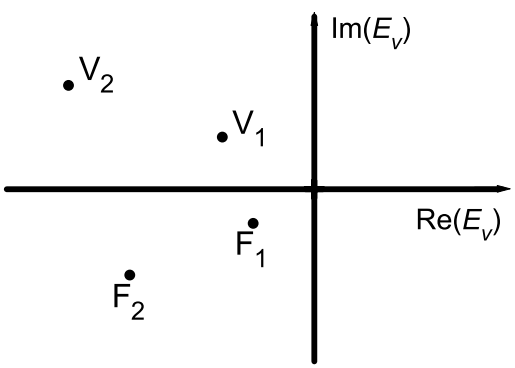

FIG. 5. Poles of the dynamical $S$ matrix describing vibrational excitation and dissociative attachment near the threshold $E_{v}=0$. The pole $F_{1}$ represents a sharp vibrational Feshbach resonance just below the threshold; the pole $\mathrm{F}_{2}$ a broader VFR farther away from the threshold. The pole $V_{1}$ represents a sharp virtual-state cusp; the pole $V_{2}$ a weaker virtual state cusp. Note that the poles $F_{1}$ and $F_{2}$ lie on the physical sheet, whereas $V_{1}$ and $V_{2}$ on the nonphysical sheet of the Riemann surface. 


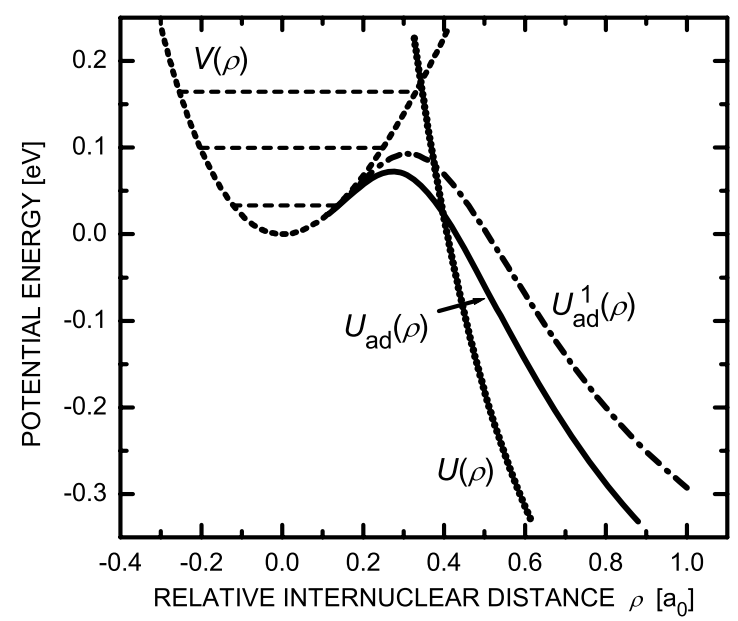

FIG. 6. Potential energy curves for a model electron scattering problem. $V(\rho)$, a curve for the neutral molecule; $U(\rho)$, the $R$-matrix pole; $U_{\mathrm{ad}}(\rho)$, the adiabatic anion curve obtained with the polarizability $\alpha=54$ a.u. and the dipole moment $\mu=0.638$ a.u. The chain curve $U_{\mathrm{ad}}{ }^{1}(\rho)$ illustrates the change of the adiabatic curve when $U(\rho)$ is shifted upwards by $\Delta E_{S}=0.1 \mathrm{eV}$. The vibrational levels of the neutral molecule are indicated by horizontal solid lines.

In the presence of a strong long-range interaction between the electron and the molecule, the adiabatic curve turns down near the crossing point and follows the neutral curve down to the internuclear distances close to equilibrium. This behavior is illustrated in Fig. 6 by choosing model potential energy curves $V(\rho)$ and $U(\rho)$ and calculating the adiabatic energy curve $U_{\mathrm{ad}}(\rho)$ for a given polarizability of the molecule $\alpha$ and its dipole moment $\mu$. The exact position of the crossing between the neutral and the adiabatic negative-ion curves depends on the details of the long-range interaction. In particular, if the dipole moment is supercritical and remains supercritical down to small internuclear distances $\rho$, the curves do not cross, although in practice the dipole-supported state eventually disappears because of rotational effects, as discussed above. But generally, such a behavior of the adiabatic anion curve leads to vibrational states which lie below, but very close to the vibrational states of the neutral molecule. Typically, lower vibrational states correspond to sharper resonances whereas for higher vibrational states we observe cusps which become weaker with increasing $v$.

We illustrate these results in Figs. 7 and 8 by presenting DA cross-sections calculated with the potential curves presented in Fig. 6. In Fig. 7 we increase polarizability that allows us to go from virtual-state cusps to sharp VFRs, 


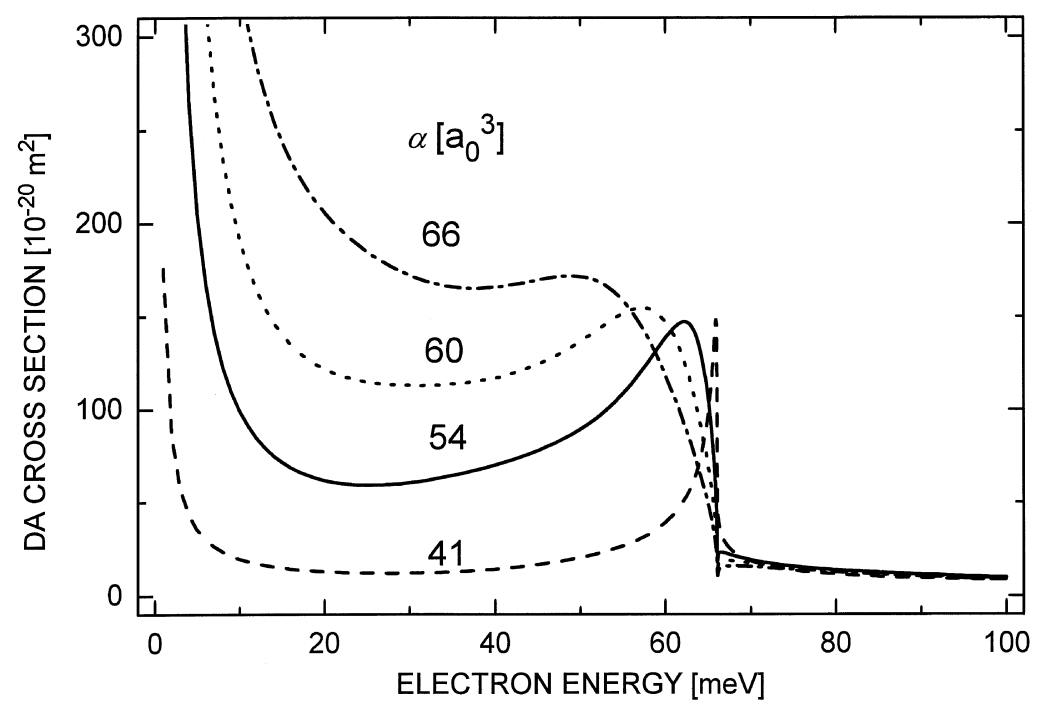

FIG. 7. Dissociative attachment cross-section obtained with potential energy curves $V(\rho)$ and $U(\rho)$ from Fig. 6 and different values of the polarizability $\alpha$ (in a.u.).

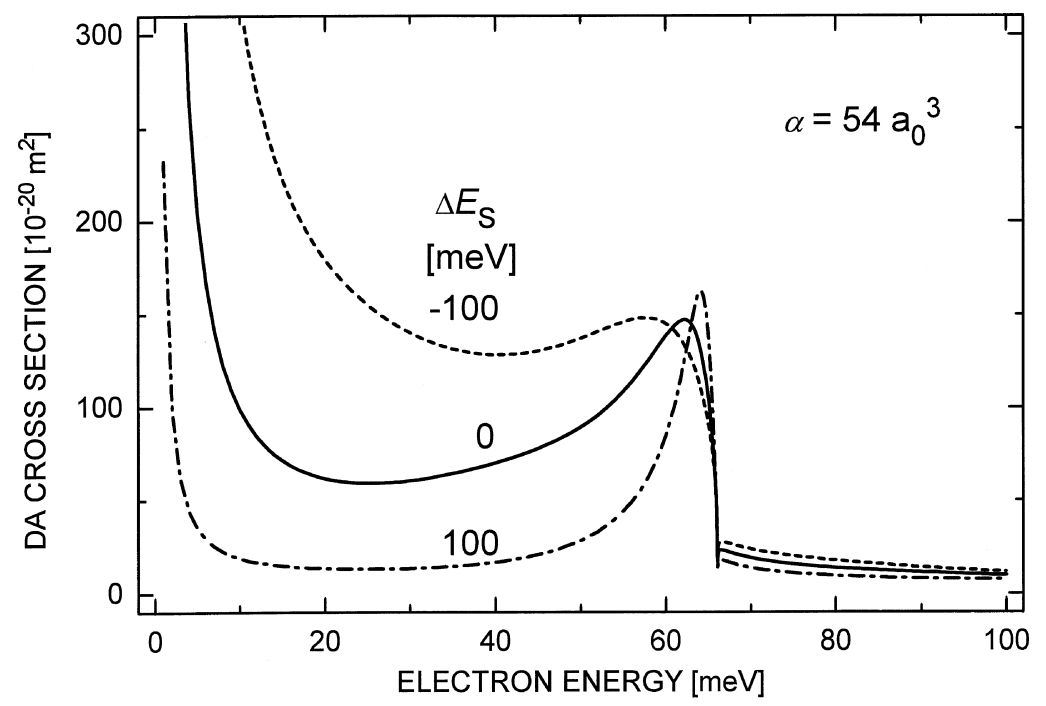

FIG. 8. Dissociative attachment cross-sections obtained from the model of Fig. 6 modified by shifting the curve $U(\rho)$ by different amounts $\Delta E_{S}$. 
and then to broader VFRs well below the threshold. Changing the dipole moment $\mu$ from 0.4 to 0.9 a.u. (while keeping the polarizability fixed at $\alpha=54$ a.u.) produces similar effects (Leber et al., 2000a). A variation of the crossing point (e.g. through shifting $U(\rho)$ by simply adding or subtracting a $\rho$-independent energy $\Delta E_{\mathrm{S}}$ ) also produces strong changes in the DA line shape. Introduction of a negative shift (lowering $U(\rho)$ ) corresponds to the solvation energy effect when the molecule is placed in a cluster or a condensed-matter environment. In Fig. 8 we show how the shape of the cusp and VFR is changing when $U(\rho)$ is shifted.

In conclusion we should stress that VFRs and cusps appear only in the dynamical $R$-matrix theory, or in the nonlocal complex potential theory which are basically equivalent. The local theory is not capable to describe the threshold effects. The effective-range-potential approximation can be considered as a limiting case of the $R$-matrix method and therefore is able to describe threshold effects, too (Gauyacq, 1982; Gauyacq and Herzenberg, 1984).

\section{B. Vogt-Wannier and Extended Vogt-Wannier Models}

A completely different approach to the description of inelastic collisions with zero-energy threshold is used in the Vogt-Wannier (VW) model for the capture into a polarization well (Vogt and Wannier, 1954). It is assumed there that the reactive process occurs with $100 \%$ probability if the electron falls into the singularity created by the polarization potential $V_{\text {pol }}=$ $-\alpha /\left(2 r^{4}\right)$. The cross-section depends only on energy and the molecular polarizability $\alpha$, and in the s-wave regime at very low energies, it is given by the simple formula

$$
\sigma_{\mathrm{VW}}(E \rightarrow 0)=4 \pi[\alpha /(2 E)]^{1 / 2} .
$$

The original VW result, Eq. (37) of Vogt and Wannier (1954), was derived from the theory of Mathieu functions. For the s-wave contribution Klots (1976) proposed a simple expression

$$
\sigma_{K}(E)=[\pi /(2 E)]\left\{1-\exp \left[-4(2 \alpha E)^{1 / 2}\right]\right\}
$$

which fits the exact Vogt-Wannier result for $l=0$ to within $8 \%$ (see Fig. 9) and describes the transition from the low-energy behavior (20) to the unitarity limit $\pi /(2 E)=\pi \lambda^{2}$ at higher energies $(\lambda=$ reduced de Broglie wavelength). 


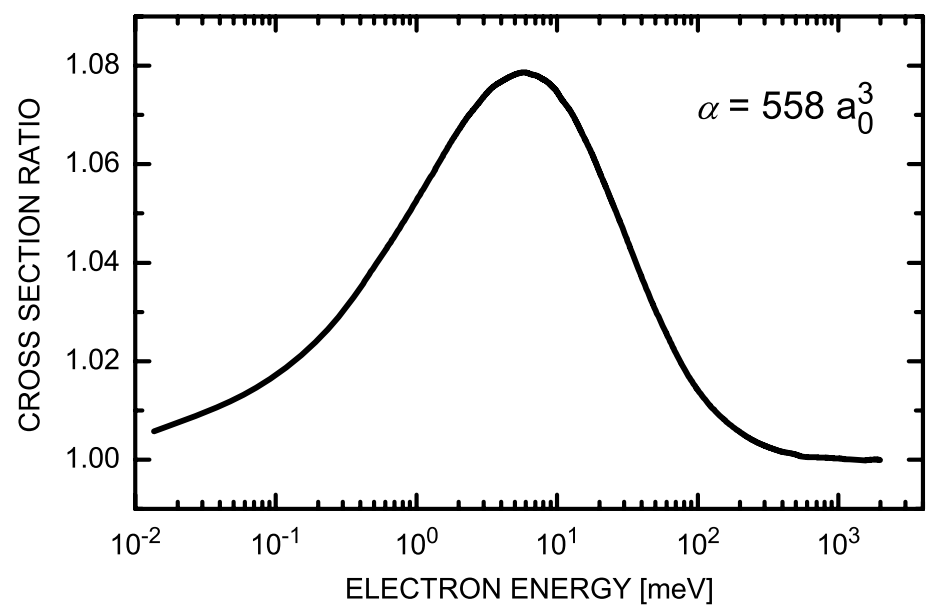

FIG. 9. Ratio $\sigma_{\mathrm{K}}(E) / \sigma_{\mathrm{VW}}(E ; l=0)$ of the approximate s-wave capture cross-section due to Klots to the s-wave capture cross-section of Vogt and Wannier for a spherically symmetric target with a polarizability of $\alpha=558$ a.u.

For molecules with high polarizabilities (such as $\mathrm{C}_{60}, \alpha\left(\mathrm{C}_{60}\right) \approx 558$ a.u., Bonin and Kresin, 1997) higher partial waves may become important even at rather low electron energies. Near zero energy the cross-section for capture of an $l \geq 1$ partial wave $\left(\sigma_{\mathrm{VWw}}(E ; l \geq 1) \propto E^{l-1 / 2}\right)$ is suppressed by the centrifugal barrier which the electron has to penetrate by tunneling. Inspection of the numerically computed partial capture cross-sections $\sigma_{\mathrm{Vw}}(E ; l \geq 1)$ shows that they exhibit a maximum at an electron energy $E_{\mathrm{max}, l}$ which is close to the maximum value $V_{\text {eff }}\left(r_{\max } ; l\right)=[l(l+1)]^{2} /(8 \alpha)$ (a.u.) of the effective potential $V_{\text {eff }}(r ; l)=-\alpha /\left(2 r^{4}\right)+l(l+1) /\left(2 r^{2}\right)$ where $r_{\text {max }, l}=[2 \alpha /(l(l+1))]^{1 / 2}$. For $\alpha=558$ a.u., the maximum values of $V_{\text {eff }}$ for $l=1,2$ and 3 are given by $24.4,219$, and $878 \mathrm{meV}$; the maxima are located at 23.6, 13.6, and $9.6 \mathrm{a}_{0}$, respectively. In Fig. 10 we show the $l=0-4$ VogtWannier capture cross-sections for electron capture by the $\mathrm{C}_{60}$ molecule. At very low energies the Wigner threshold behaviour $\left(\propto E^{l-1 / 2}\right)$ is observed while towards high energies the respective unitary limit $\sigma_{l}=\pi(2 l+1) / k^{2}$ is reached. It is interesting to note (see also Vogt and Wannier, 1954; Klots and Compton, 1996) that the total VW cross-section $\sigma_{\mathrm{VW}, \text { tot }}(E)$ is found to agree with the classical Langevin cross-section $\sigma_{\text {Lang }}(E)=2 \pi[\alpha /(2 E)]^{1 / 2}$ (Langevin, 1905) to within $5 \%$ for energies above about $4 \mathrm{meV}$ (more generally for $\alpha E>0.08$ a.u.). Towards lower energies the ratio $\sigma_{\mathrm{Vw} \text {,tot }}(E) /$ $\sigma_{\text {Lang }}(E)$ continuously rises towards the value 2 which is reached in the limit of zero energy, as illustrated in Fig. 11. 


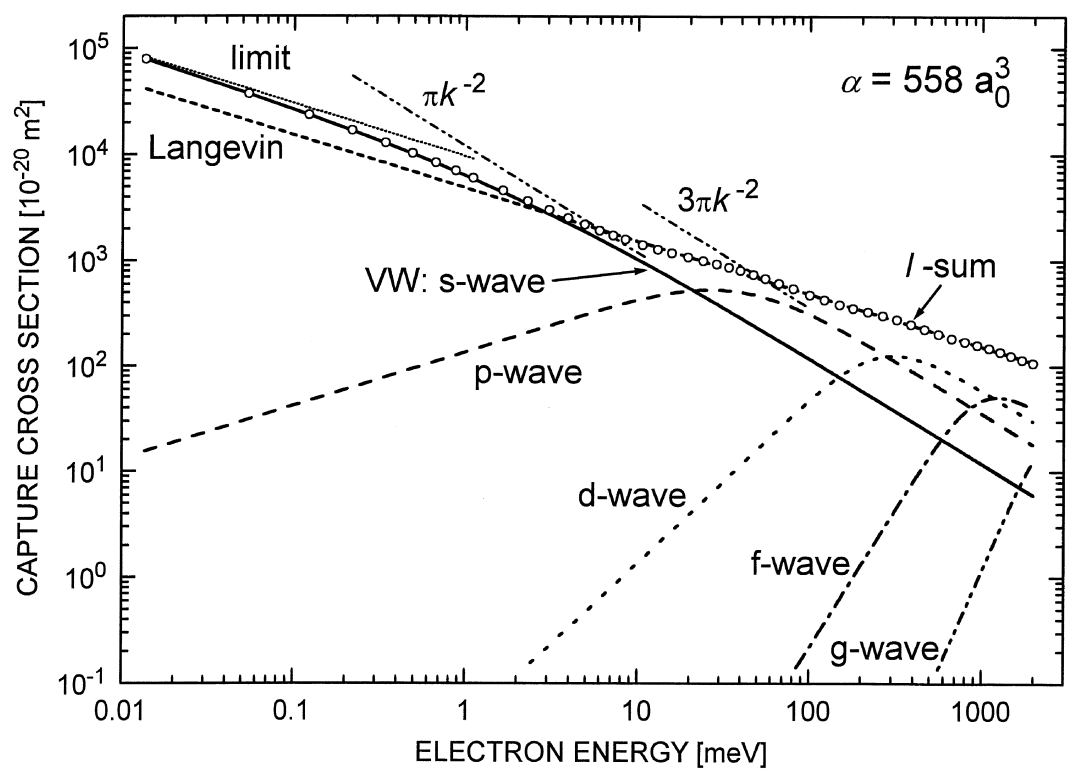

FIG. 10. Partial $l=0-4 \mathrm{VW}$ cross-sections and the resulting total VW cross-section (open circles) for electron capture by a spherically symmetric target with a polarizability of $\alpha=558$ a.u. In addition the figure presents the limiting s-wave Vogt-Wannier capture cross-section Eq. (20) (dotted line), the classical Langevin cross-section (short dashes) as well as the unitary limits $\pi k^{-2}$ and $3 \pi k^{-2}$ for reactive s-wave and p-wave scattering, respectively.

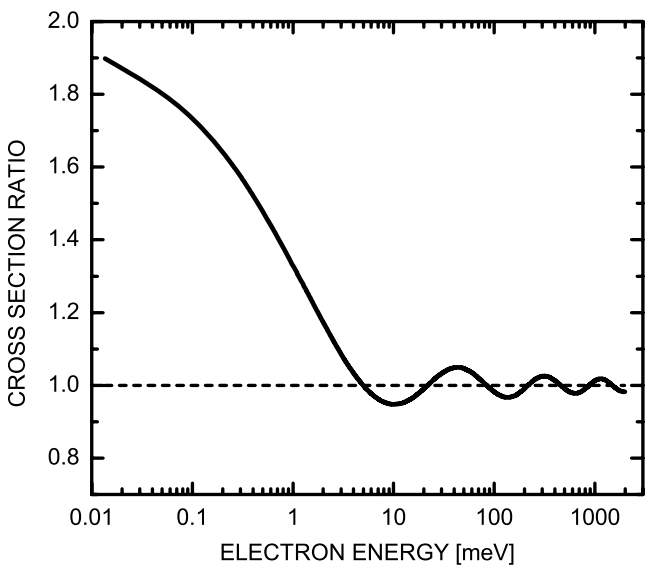

FIG. 11. Energy dependence of the ratio of the total Vogt-Wannier capture cross-section to the classical Langevin capture cross-section for a spherically symmetric target with a polarizability of $\alpha=558$ a.u. 
The Vogt-Wannier (VW) model seems to be unphysical in the sense that the actual long-range potential does not have a $r^{-4}$ singularity. Although both Eq. (20) and the resonance theory give the same energy dependence for s-wave electrons, there is no relation between them otherwise. The VW model does not incorporate the resonance mechanism, therefore there is no resonance characteristics like a width in its equation.

The situation turns out to be even more complicated for dipolar molecules. If the electron energy is large compared to the rotational spacing (an assumption which holds down to sub-meV energies for relatively heavy molecules), the Bethe-Wigner threshold law should be modified (Fabrikant, 1977). For subcritical dipole moments, $\mu<\mu_{c r}=0.6395$ a.u., the crosssection becomes proportional to $E^{\lambda-1 / 2}$ where $\lambda$ is a threshold exponent whose value varies between 0 for $\mu=0$ and $-1 / 2$ (for $\mu=\mu_{c r}$ ). The resonance theory is consistent with this modification.

An extension of the VW theory for polar targets was given by Fabrikant and Hotop (2001). We will outline briefly the approach which they used. It follows closely the original derivation of Vogt and Wannier. The VW theory assumes the absorption boundary condition at the origin due to capture into the polarization well. The reaction cross-section $\sigma_{\mathrm{r}}$ in this case is given by (Landau and Lifshitz, 1977)

$$
\sigma_{r}=\frac{\pi}{k^{2}} \sum_{l l^{\prime}}\left(\delta_{l l^{\prime}}-\left|S_{l l^{\prime}}\right|^{2}\right),
$$

where $S_{l l^{\prime}}$ are the matrix elements of the scattering operator in the angular momentum representation.

The Schrödinger equation for a superposition of the dipolar and polarization potentials allows separation of the variables. The wavefunction can be expanded in the dipolar angular harmonics (Mittleman and von Holdt, 1965) and the radial equation has the form

$$
\left(\frac{d^{2}}{d r^{2}}+k^{2}-\frac{\lambda(\lambda+1)}{r^{2}}+\frac{\alpha}{r^{4}}\right) u(r)=0,
$$

where $k^{2}=2 E$. For subcritical dipole moments considered here $\lambda(\lambda+1)>$ $-1 / 4$ and $\lambda$ is real.

The scattering matrix can also be transformed into the dipolar angular harmonics representation where it becomes diagonal. In the low-energy region only the lowest eigenvalue $\lambda$ makes a contribution to the inelastic 
cross-section which can now be written in the form

$$
\sigma_{r}=\frac{\pi}{k^{2}}\left(1-\left|S_{0}\right|^{2}\right)
$$

where $S_{0}$ is the matrix element of the scattering operator corresponding to the lowest $\lambda$.

The required solution of the radial equation with the ingoing-wave boundary conditions at the origin has a simple analytical form in the low-energy region (Fabrikant, 1979). For $S_{0}$ we obtain

$$
S_{0}=\frac{1 / b-b}{1 / b+b \exp (-2 \pi i \lambda)},
$$

and

$$
1-\left|S_{0}\right|^{2}=\frac{4 \cos ^{2} \pi \lambda}{b^{2}+1 / b^{2}+2 \cos 2 \pi \lambda}
$$

where $b^{2}=\left[\left(\Gamma(1-\lambda-1 / 2) /\left(2^{2 \lambda+1}(\Gamma(1+\lambda+1 / 2))\right]^{2}\left(\alpha^{1 / 2} k\right)^{2 \lambda+1}\right.\right.$, and $\lambda(\lambda+1)$ is an eigenvalue of the operator $L^{2}-2 \mu \cos \theta$ (see above). Note that although $b$ is asymptotically small, the threshold exponent $\tau$ might be close to 0 , as, for example, in the case of $\mathrm{CH}_{3} \mathrm{I}$, therefore Eqs. (25), (26) should not be simplified further. In particular, using $1-\left|S_{0}\right|^{2}=4 b^{2} \cos ^{2} \pi \lambda$ for the $\mathrm{CH}_{3} \mathrm{I}$ molecule violates the unitarity limit even at the electron energy $E=0.01 \mathrm{meV}$.

For a target with a given polarizability $\alpha$, a sub-critical dipole moment $\left(\mu<\mu_{\mathrm{c}}, \mu_{\mathrm{c}}=0.6395\right.$ a.u. $\left.=1.625 \mathrm{D}\right)$ and at electron energies sufficiently high to view the molecular rotation as frozen, we thus obtain the s-wave capture cross-section (labelled 'Extended Vogt-Wannier', EVW) in the form

$$
\sigma_{\mathrm{EVW}}(E)=\left(\pi k^{-2}\right)\left[4 \cos ^{2} \pi \lambda\left(b^{2}+1 / b^{2}+2 \cos 2 \pi \lambda\right)^{-1}\right]
$$

For dipole moments in the range $0 \leq \mu<\mu_{c}$ and $l=0, \lambda$ takes values in the range $0 \geq \lambda>-1 / 2$; the relation between $\lambda$ and $\mu$ is to a good approximation described by the equation ( $\mu$ in atomic units) (Klar et al., 2001b)

$$
\lambda(\mu)=-(1 / 2)+(1 / 2)\left[1-4 \mu^{2}\left(0.66655-0.15646 \mu^{2}+0.050418 \mu^{4}\right)\right]^{1 / 2} .
$$


Correspondingly, the cross-section (27) exhibits an energy dependence $\sigma_{\mathrm{EVW}}(E) \propto E^{-X}$ with $0.5<X<1$. At fixed polarizability $\alpha$, the absolute values of the EVW cross-section (27) for nonzero dipole moment exceeds the Vogt-Wannier cross-section. The EVW formula (27) is a reasonable approximation to the exact cross-section for electron capture by combined polarization and dipolar forces for electron energies lower than $0.25 / \alpha$ (a.u.). For zero dipole moment, formula (27) represents a good approximation to the exact s-wave VW cross-section for energies such that $\alpha E<2$ a.u. (deviations $<11 \%$ ); towards higher energies formula (27) attains too low values and does not join the unitary limit while the Klots formula (21) remains a good approximation from low to high energies.

It should be noted, however, that the EVW expression (27) as well as the original VW and the Klots formula can be considered only as an estimate of the capture cross-section in the s-wave regime since the resonance mechanism and the actual nuclear motion (survival probability in the anion state) are not included. Typically, the VW and EVW formulae work well if the negative-ion curve crosses the neutral curve in the vicinity of the minimum of the latter, i.e. in the case of a favourable Franck-Condon factor (which, however, does not enter the EVW formula), as for the $\mathrm{CCl}_{4}$ (Klar et al., 2001a) and the $\mathrm{CFCl}_{3}$ molecule (Klar et al., 2001b). Another limitation of EVW/VW theory is related to the coupling of the attachment process to vibrationally inelastic channels. As a result, the EVW/VW crosssection is expected to be valid only up to the first threshold for excitation of vibrational levels whose symmetry allows strong coupling to the s-wave attachment process.

In Fig. 12 we present the DA cross-section for methyl iodide molecules in the vibrational ground state $\mathrm{CH}_{3} \mathrm{I}\left(v_{3}=0\right)$, calculated using different theories: the Klots fit of the VW result, the EVW (including the dipole moment) result, the complete $R$-matrix calculation and the results of the local complex potential (LCP) approximation. The equation for the DA cross-section in the LCP approximation was derived from the nonlocal theory by O'Malley (1966) and Bardsley (1968), and can be summarized (for a nondegenerate doublet resonance) as:

$$
\sigma_{\mathrm{DA}}(E)=\left(2 \pi^{2} / k^{2}\right) \Gamma|\mathrm{FC}|^{2} S
$$

where $\Gamma$ is the resonance width, $S$ is the survival factor, and FC denotes the Franck-Condon overlap between the initial vibrational level of the neutral molecule and the continuum nuclear wave function in the dissociative resonance anion state, normalized to the delta function of energy. Note that with this normalization $|\mathrm{FC}|^{2}$ has the dimension energy ${ }^{-1}$, and we do not 


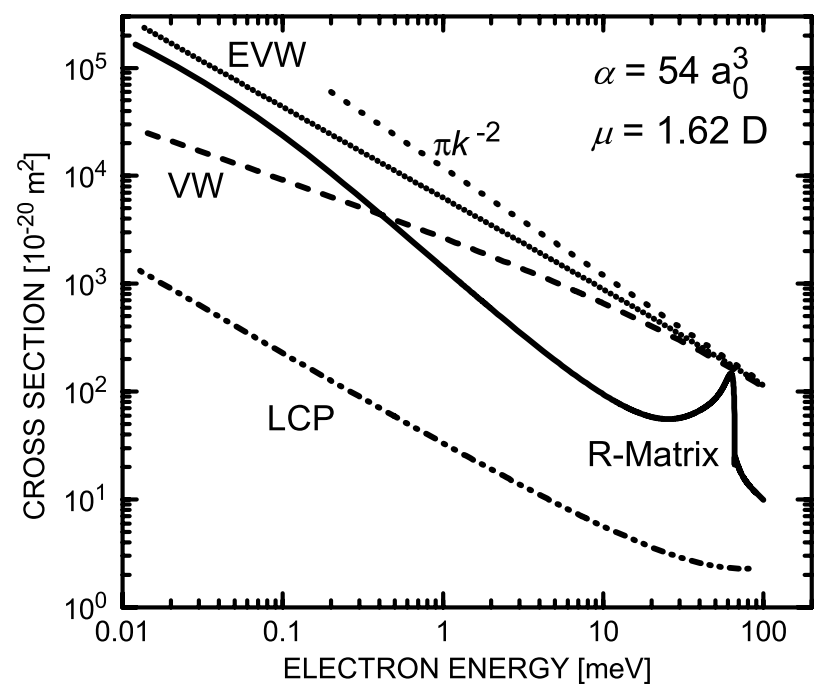

FIG. 12. Illustration of the EVW result compared with the VW result for s-wave attachment to $\mathrm{CH}_{3} \mathrm{I}\left(\alpha=54 \mathrm{a}_{0}{ }^{3}, \mu=1.62 \mathrm{D}\right)$ and comparison with the $R$-matrix result and LCP theory (see Fig. 1 in Fabrikant and Hotop, 2001).

use O'Malley's delta function approximation for the dissociating state. The calculation of FC was done, however, assuming that the energy of the dissociating state is real.

Large deviations between the different theories are observed in Fig. 12, reaching two orders of magnitude at a fixed energy and with the lowest cross sections obtained with the local theory and the highest with the EVW. The energy dependence of the cross-section at ultralow energies is determined by the threshold exponent $(\lambda-1 / 2)$ which is -0.965 for $\mathrm{CH}_{3} \mathrm{I}$. Therefore both the extended Vogt-Wannier model and the local theory predict a fast growth of the cross-section towards zero energy, approaching $E^{-0.965}$. However, the nonlocal $R$-matrix results above $0.1 \mathrm{meV}$ exhibit an even faster variation. In addition, the nonlocal cross-sections are much greater (typically almost two orders of magnitude) than those of the local calculations, in agreement with the experiment. The $R$-matrix cross-section near the threshold for vibrational excitation of the symmetric $\mathrm{C}-\mathrm{I}$ stretch is dominated by the vibrational Feshbach resonance which was discussed above in Section II.A.4. All other calculations do not exhibit this resonance. The strong enhancement (as compared to the local approximation) of the DA cross-section close to zero energy is caused by the same weakly bound state which supports the VFR near the $v_{3}=1$ threshold (Fabrikant and 
Hotop, 2001). The analytical expression, describing this enhancement for a molecule with a subcritical dipole moment, can be written as (Fabrikant, 1977, 1978)

$$
\sigma=c k^{2 \lambda-1} /\left|\eta-i k^{2 \lambda+1} \exp (-i \pi \lambda)\right|^{2},
$$

where $\eta$ is a complex parameter. This is similar to Eq. (12) written for a supercritical dipole moment. This expression has a pole in the complex $k$ plane corresponding to a bound or a virtual state, if $\eta$ is real. Because of the coupling with the DA channel this state, even if it is bound, can decay. However, the decay width is small because of the potential barrier towards dissociation. The width of the VFR below the $v_{3}=1$ threshold is substantially larger because of the lower potential barrier for the nuclear motion in the $v_{3}=1$ state. The resonance disappears at $v_{3}=2$. In summary, the big value of the DA cross-section for methyl iodide in the ultra-low energy region can be explained by the influence of the dipole-supported state which is not incorporated into the local version of the resonance theory.

\section{Experimental Aspects}

In this section we discuss three different experimental setups for studies of electron-molecule collisions at low energies with high resolution $(<10 \mathrm{meV})$. We note that most low-energy electron collision studies in the gas phase have been and are being carried out at broader energy widths (typically in the range $30-150 \mathrm{meV}$ ), e.g. using trochoidal electron monochromators (TEM) which - by virtue of the magnetic guiding field - rather easily allow studies down to zero energy and - in conjunction with a TEM for energy analysis - also provide access to investigations of inelastic scattering with high sensitivity (Allan, 1989).

\section{A. Setur Involving Angle- and Energy-Resolved Detection of Scattered Electrons}

A typical apparatus for studies of angle-dependent elastic and inelastic scattering consists of a hot filament electron source followed by an electrostatic monochromator, a target beam (of either effusive or supersonic character) and an angle-variable electrostatic energy analyzer (Allan, 1989; Brunger and Buckman, 2002). Using such an optimized instrument, as shown in Fig. 13, Allan has recently achieved energy widths down to $7 \mathrm{meV}$ at high signal to background ratio in energy loss spectra and excitation 


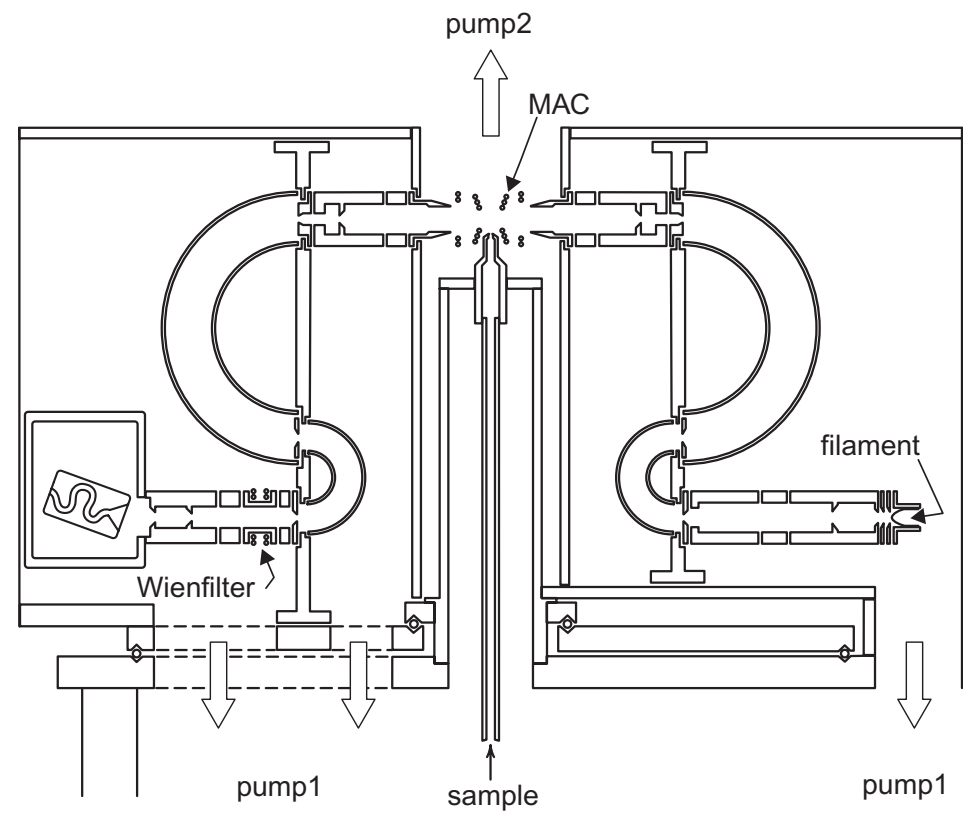

FIG. 13. The Fribourg apparatus for angle-differential low-energy electron scattering from a gaseous target beam. A small Wien-type mass filter is used to separate scattered electrons and fragment anions from dissociative attachment. The analyzers and the electron optics are differentially pumped as indicated by the arrows. The dots around the collision region (labelled MAC) represent the 'magnetic angle-changing device' (seen in cross-section).

functions involving elastic and vibrational inelastic scattering (Allan, 2001a; Allan and Moreira, 2002; Skalický et al., 2002). Through careful calibration procedures absolute differential cross-sections are obtained.

Using optimized conventional electron sources, Ibach's group (1991, 1993) have achieved energy widths down to $1 \mathrm{meV}$ in electron scattering from molecules adsorbed at surfaces under ultrahigh vacuum and low current conditions.

Photoelectron sources have been used for angle differential gas phase scattering experiments by Gallagher and coworkers (van Brunt and Gallagher 1978; Kennerly et al., 1981), Field et al. (1988, 1991a,b) and, more recently, by Gopalan et al. (2003). In order to avoid the Doppler effect, differentially-pumped supersonic beam targets were used (see, e.g., Götte et al., 2000). In principle an energy width down to $1 \mathrm{meV}$ should be achievable at electron currents around $50 \mathrm{pA}$ (Bömmels et al., 2001; Gopalan et al., 2003), but the full potential of this approach has yet to be demonstrated and exploited for angle-differential scattering experiments. 
A long-standing problem, namely the detection of electrons scattered into angles around $180^{\circ}$, was recently solved by the introduction of 'magnetic angle-changing devices' by Read and coworkers (Read and Channing 1996; Zubek et al., 1996). They typically consist of two Helmholtz-type pairs of current coils (coaxial solenoids around a common axis) and are designed such that the resultant magnetic field is zero in the outer region (allowing to keep electrostatic energy selectors) and nonzero in the region around the beam target. So far, the method has been applied mainly to electron scattering from rare gas atoms (e.g. Cubric et al., 1999; Zubek et al., 1999, 2000; Allan, 2000). An elegant version which is well suited for electron scattering studies involving collimated supersonic beams has been implemented by Allan (2000).

\section{B. Measurement of Total Scattering Cross-Sections}

Experimental setups for the determination of total electron scattering crosssections (comprising elastic as well as all inelastic collisions including attachment processes) involve a well-collimated electron beam of variable energy which is transmitted through a collision cell containing the static target gas. Non-scattered electrons are detected within a narrow angular range in the forward direction. Some energy analysing device is included to prevent inelastically forward scattered electrons from being detected; normally, however, the resolution is not sufficiently high to exclude electrons which have undergone rotational energy losses in the forward scattering. This aspect is critical for molecules with dipole moments. The energy resolution in these experiments is limited by the energy width of the electron source $\left(\Delta E_{\mathrm{S}}\right)$, by the potential variations in the target region $\left(\Delta E_{\mathrm{T}}\right)$, and ultimately by the Doppler effect $\left(\Delta E_{\mathrm{D}}\right)$ due to the random motion of the target molecules (mass $m_{\mathrm{T}}$, average velocity $\mathbf{v}_{\mathrm{T}}$, kinetic energy $E_{\mathrm{T}}$ ) with respect to the directed electron beam (mass $m_{\mathrm{e}}$, velocity $\mathbf{v}_{\mathrm{e}}$, kinetic energy $E_{\mathrm{e}}$ ). The collision energy $E$ of the electron-molecule system in the center-of-mass frame is given by

$$
E \approx E_{\mathrm{e}}-\left(m_{\mathrm{e}} / m_{\mathrm{T}}\right) E_{\mathrm{e}}-2\left(m_{\mathrm{e}} E_{\mathrm{e}} E_{\mathrm{T}} / m_{\mathrm{T}}\right)^{1 / 2} \cos \theta
$$

where $\theta$ is the angle between $v_{\mathrm{e}}$ and $v_{\mathrm{T}}$. In (31) the second term is the recoil energy which can be neglected at low collision energies. The third term is the energy shift $E_{\mathrm{D}}$ due to the first order Doppler effect. Here we simply estimate the Doppler energy width $\Delta E_{\mathrm{D}}$ by

$$
\Delta E_{\mathrm{D}}=(1 / 2)\left[E_{\mathrm{D}}\left(180^{\circ}\right)-E_{\mathrm{D}}\left(0^{\circ}\right)\right]=2\left(m_{\mathrm{e}} E_{\mathrm{e}} E_{\mathrm{T}} / m_{\mathrm{T}}\right)^{1 / 2}
$$




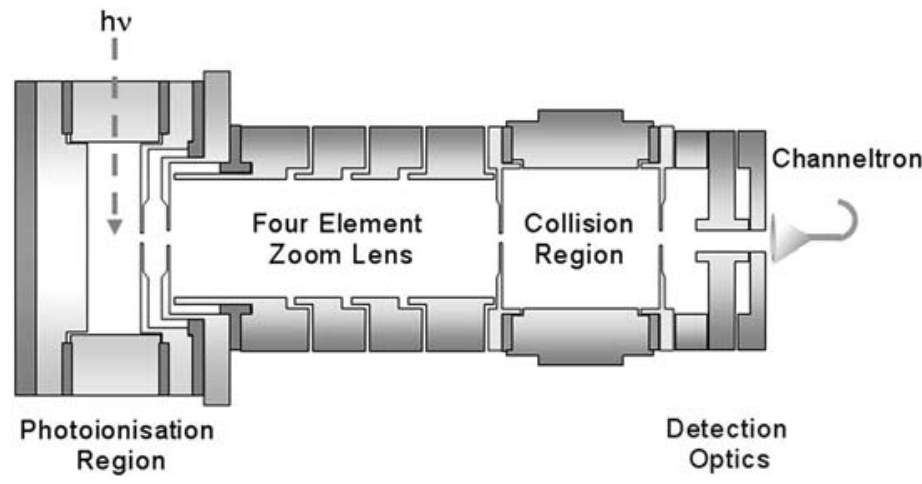

FIG. 14. Schematic diagram of the apparatus for studies of total electron scattering cross sections involving a VUV photoelectron source (from Hoffmann et al., 2002).

For electron energies below $1 \mathrm{eV}$ and thermal targets $\left(T_{\mathrm{T}}=300 \mathrm{~K}\right)$ with a mass above $28 \mathrm{u}$ the Doppler width stays below $2 \mathrm{meV}$, but is has to be taken into account when (sub) $\mathrm{meV}$ resolution is to be achieved.

In Fig. 14 we show an apparatus (Hoffmann et al., 2002) which has been used in this or similar form by Field and Ziesel with coworkers (Field et al., 1991a; Ziesel et al., 1993; Gulley et al., 1998a, b; Field et al., 2000; Lunt et al., 2001; Field et al., 2001a,b,c; Jones et al., 2002; Ziesel et al., 2003) to investigate total electron scattering cross-sections for a large number of molecules from low (about $20 \mathrm{meV}$ ) to medium electron energies (around $10 \mathrm{eV}$ ). Electrons are created in the source region by photoionization of ground state argon atoms (at a pressure of a few tens of $\mathrm{mPa}$ ) through the narrow $\operatorname{Ar}\left(11 \mathrm{~s}^{\prime}, J=1\right)$ resonance at $78.65 \mathrm{~nm}$ (about $4 \mathrm{meV}$ above the photoionization threshold), using focussed $(10-20 \mu \mathrm{m})$ monochromatized synchrotron radiation. The photoelectrons (current up to $1 \mathrm{pA}$ ) are extracted by a weak electric field $\left(20-40 \mathrm{~V} \mathrm{~m}^{-1}\right)$ and formed into a focussed beam with a four-element electrostatic lens. The energy width of this beam is determined mainly by the ionizing photon bandwidth; in the earlier experiments at SuperACO (LURE, Orsay, FR) and SRS (Daresbury, UK) the photon energy width amounted typically to $5-6 \mathrm{meV}$ while in the recent experiments at ASTRID (Aarhus, DK) widths around $1 \mathrm{meV}$ were achieved (Field et al., 2000, 2001a, b; Hoffmann et al., 2002).

The electron beam passes through the collision chamber which contains the target gas at a known number density $\rho_{\mathrm{T}}$. The beam attenuation as a function of electron energy is measured by recording the electron current on a channel electron multiplier situated beyond further optical elements. The electron energy is scanned by varying the potential in the photoionization 
source. The whole apparatus can be immersed in an axial magnetic field (typically $2 \mathrm{mT}$ ), thus allowing the determination of cross-sections for backward scattering to be compared with the total cross-sections, measured in the absence of magnetic fields. Absolute cross-sections $\sigma$ are evaluated using Beer's law

$$
I_{\mathrm{t}}=I_{0} \exp \left(-\sigma(E) \rho_{\mathrm{T}} z\right)
$$

where $I_{\mathrm{t}}$ and $I_{0}$ are the attenuated and the unattenuated electron currents, respectively, $\sigma(E)$ is the enery dependent scattering cross-section, and $z$ is effective electron path length in the target gas. Uncertainties for the absolute values of the cross-sections are around $10 \%$. The absolute energy scale is calibrated to within $\pm 5 \mathrm{meV}$. Under optimum conditions cross-sections are determined at energies down to $10 \mathrm{meV}$. Measurements of backward cross-sections are limited to incident energies below $650 \mathrm{meV}$; this limit is imposed by the size of the exit hole $(3 \mathrm{~mm})$ of the scattering chamber in conjunction with the value of the guiding axial magnetic field $(2 \mathrm{mT})$ : above $650 \mathrm{meV}$, forward scattering will contribute to the measured cross-section.

\section{Measurement of Cross-Sections for Electron Attachment}

The use of VUV photoelectron sources for high resolution studies of electron attachment to molecules has been initiated by Chutjian and coworkers (Ajello and Chutjian, 1979; Chutjian and Alajajian, 1985) under the acronym TPSA (Threshold Photoelectron Spectroscopy for Attachment). In these experiments, rare gas atoms $\mathrm{Rg}(\mathrm{Rg}=\mathrm{Kr}$ or $\mathrm{Xe})$ were photoionized above the second ionization threshold (formation of $\operatorname{Rg}^{+}\left({ }^{2} \mathrm{P}_{1 / 2}\right)+$ $\left.\mathrm{e}^{-}\left(E_{\mathrm{e}}\right)\right)$ to create electrons with variable energy by tuning the wavelength of monochromatized VUV radiation from a Hopfield continuum light source. By choosing this ionization path, higher energy electrons are simultaneously created due to formation of $\operatorname{Rg}^{+}\left({ }^{2} \mathrm{P}_{3 / 2}\right)$ ions. The contribution of these electrons to the attachment signal is only negligible as long as the attachment cross-section drops sufficiently rapidly with increasing electron energy. The source volume contained both the rare gas atoms and the target molecules as static gases at rather high densities (about $0.01 \mathrm{~Pa}$ and $0.45 \mathrm{~Pa}$, resp.). Negative ions, resulting from electron attachment to the molecules, were extracted with a weak electric field and detected with a quadrupole mass spectrometer. Attachment spectra for a large number of molecules were thus obtained over the energy range $0-160 \mathrm{meV}$ (Chutjian, 1992; Chutjian et al., 1996). The energy resolution in the TPSA experiments was limited by the photon bandwidth (typically $6-8 \mathrm{meV}$ ) and by the 
extraction field. When the wavelength is set close to the $\operatorname{Rg}^{+}\left({ }^{2} P_{1 / 2}\right)$ threshold the interpretation of the attachment signals may get complicated by the fact that autoionizing Rydberg resonances $\operatorname{Rg}^{* * *}\left({ }^{2} \mathrm{P}_{1 / 2} n \mathrm{~s}^{\prime}, n \mathrm{~d}^{\prime}\right)$ are created apart from free electrons. At principal quantum numbers $n$ above about 150 , the lifetime of these resonances may become sufficiently long by field- and collision-induced mixing with high angular momentum states that contributions to the anion signal due to Rydberg electron transfer cannot be ruled out (Klar et al., 1994a). Recently, Chutjian and coworkers have used an improved TPSA setup in which coherent VUV radiation, produced by frequency mixing techniques involving pulsed narrow-band (sub-meV) lasers, is used to photoionize $\mathrm{Xe}$ atoms above the $\mathrm{Xe}^{+}\left({ }^{2} \mathrm{P}_{1 / 2}\right)$ threshold (Howe et al., 2001). The Xe atoms are provided in conjunction with the target molecules as a collimated pulsed seeded supersonic beam. The experiment has the drawback of rather low pulse repetition rate (about $10 \mathrm{~s}^{-1}$ ) which makes it difficult to achieve good statistical quality of the data and to follow attachment cross-sections over several orders of magnitude.

A powerful variant of the TPSA method has been developed by Klar et al. (1992a, b, 1994a) which produces monoenergetic electrons by photoionization of laser-excited ${ }^{40} \mathrm{Ar}^{*}\left(4 \mathrm{p}^{3} \mathrm{D}_{3}\right)$ atoms (Klar et al., 1994a; Schohl et al., 1997) or laser excited ${ }^{39} \mathrm{~K}^{*}\left(4 \mathrm{p}_{3 / 2}\right)$ atoms (Weber et al., 1999a, b; Petrov et al., 2000 ) with a tunable blue dye laser of narrow bandwidth (typically $0.15 \mathrm{meV}$ or $0.05 \mathrm{meV}$ ). These laser photoelectron sources have been used in a series of laser photoelectron attachment (LPA) studies involving molecules and molecular clusters. A recent version of the experimental setup is shown schematically in Fig. 15 (Weber et al., 1999b; Barsotti et al., 2002a).

For the first time electron collision experiments in the gas phase have been thus carried out at sub-meV resolution (Klar et al., 1992a, b) and at incident energies down to $20 \mu \mathrm{eV}$ (Schramm et al., 1998). While the excited atoms are provided at low density (about $2 \times 10^{6} \mathrm{~cm}^{-3}$ for $\operatorname{Ar}^{*}\left(4 \mathrm{p}^{3} \mathrm{D}_{3}\right), 10^{8} \mathrm{~cm}^{-3}$ for $\mathrm{K}^{*}\left(4 \mathrm{p}_{3 / 2}\right)$ ) in a collimated atomic beam, the continuous ionizing intracavity laser is sufficiently intense (power $1-5 \mathrm{~W}$ ) to achieve typical currents around $1 \mathrm{pA}\left(\mathrm{Ar}^{*}\right)$ or $50 \mathrm{pA}\left(\mathrm{K}^{*}\right)$. The two-step ionization path, involving efficient primary laser excitation of metastable ${ }^{40} \operatorname{Ar}\left(4 \mathrm{~s}^{3} \mathrm{P}_{2}\right)$ and ground state.

${ }^{39} \mathrm{~K}\left(4 \mathrm{~s}_{1 / 2}, \mathrm{~F}=1,2\right)$ atoms to the intermediate levels ${ }^{40} \mathrm{Ar}^{*}\left(4 \mathrm{p}^{3} \mathrm{D}_{3}\right)$ or ${ }^{39} \mathrm{~K}^{*}\left(4 \mathrm{p}_{3 / 2}, \mathrm{~F}^{\prime}=2,3\right)$ (quasi-stationary excited state population nearly $50 \%$ ), is optimized in the sense that the cross-section for ionization of $\operatorname{Ar}^{*}\left(4 \mathrm{p}^{3} \mathrm{D}_{3}\right)$ (Schohl et al., 1997) as well as for $\mathrm{K}^{*}\left(4 \mathrm{p}_{3 / 2}\right)$ atoms (Petrov et al., 2000) is substantial (around $10^{-21} \mathrm{~m}^{2}$ ) and three to four orders of magnitude higher than that for ionization of metastable $\operatorname{Ar}^{*}\left(4 \mathrm{~s}^{3} \mathrm{P}_{2}\right)$ atoms (Kau et al., 1998; Petrov et al., 1999) or ground state K(4s) atoms (Sandner et al., 1981), respectively. We note that an analogous two-step photoionization scheme 


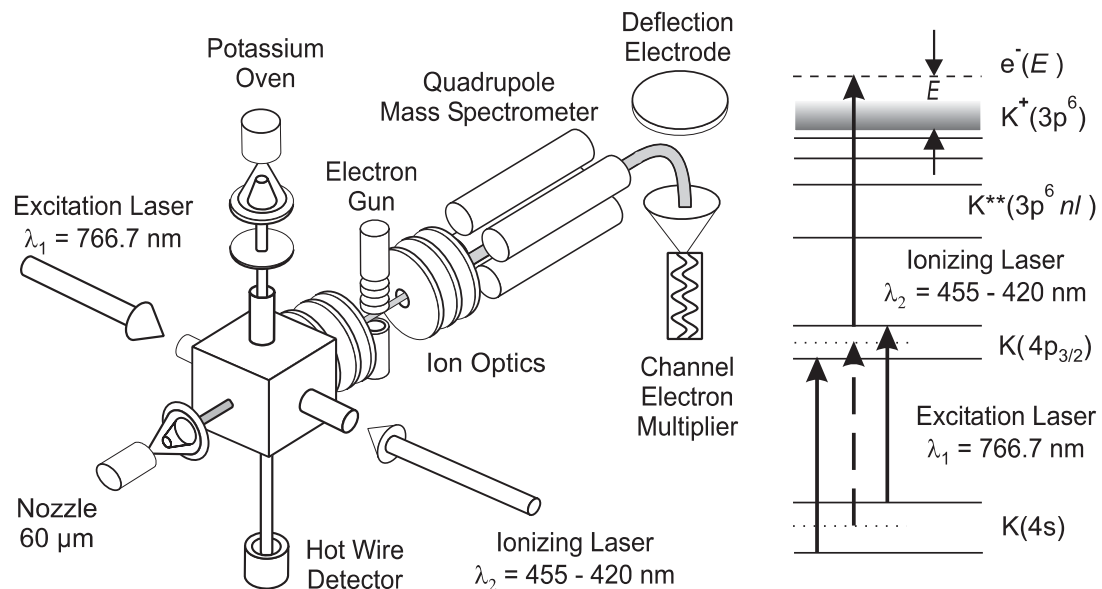

FIG. 15. Laser photoelectron attachment experiment involving two-step photoionization of potassium atoms, a supersonic target beam (differentially pumped nozzle) and mass spectrometric detection of the anions. The auxiliary electron gun is used for diagnostics of the target beam and of the residual gas by means of electron impact ionization. The laser photoelectron production scheme is shown on the right side (after Weber et al., 2000).

involving ground state $\mathrm{Na}\left(3 \mathrm{~s}_{1 / 2}\right)$ atoms has been recently applied by Keil et al. (1999) to study laser photoelectron attachment to vibrationally excited $\mathrm{Na}_{2}$ molecules (see Section IV.B). The use of an ionizing laser at rather long visible wavelengths in all these two-step ionization schemes is advantageous for several reasons: (i) the high continuous laser intensity, available with an intracavity Stilbene 3 dye laser, allows substantial photoelectron currents to be produced from a thin atomic target; (ii) the bandwidth of the laser can be made sufficiently narrow (e.g. by using a single mode laser) to allow in situ diagnostics of residual dc electric fields in the photoionization/attachment volume by studies of the Stark effect (Frey et al., 1993; Osterwalder and Merkt, 1999) or of the shift of the ionization threshold (Klar et al., 1994a; Schramm et al., 1998); (iii) the production of electrons emitted from surfaces by scattered laser light is negligible in the LPA experiment (wavelengths around $450 \mathrm{~nm}$ ) while it is difficult to avoid in the TPSA experiment at VUV wavelengths (Howe et al., 2001).

Using laser photoionization of $\operatorname{Ar}^{*}\left(4 \mathrm{p}^{3} \mathrm{D}_{3}\right)$ atoms over the range $462-433 \mathrm{~nm}$, Klar et al. (1992a, b, 1994a, b, 2001a, b) and Schramm et al. $(1998,1999,2002)$ have carried out the first series of LPA experiments at (sub) $\mathrm{meV}$ resolution over the typical energy range $0.2-173 \mathrm{meV}$ for selected molecules $\left(\mathrm{SF}_{6}, \mathrm{CCl}_{4}, \mathrm{CFCl}_{3}, \mathrm{HI}, \mathrm{CH}_{3} \mathrm{I}, \mathrm{CH}_{2} \mathrm{Br}_{2}, \mathrm{CCl}_{3} \mathrm{Br}, 1,1,1-\mathrm{C}_{2} \mathrm{Cl}_{3} \mathrm{~F}_{3}\right)$, 
present in the photoionization region as a static gas. In order to achieve a sufficient anion detection efficiency without the loss of energy resolution, the experiment was pulsed at high repetition rates (up to $140 \mathrm{kHz}$ ), alternating between the electron production/attachment period and the anion extraction interval, as described in detail in Klar et al. (1992b, 1994a, 2001a, b) and Schramm et al. (1999, 2002). Mass analysis was performed with a quadrupole filter (Klar et al., 1992a, b, 1994a, 2001a, b) or with a time-of-flight spectrometer (Schramm et al., 1999, 2002).

A more recent version of the LPA experiment (Fig. 15) is based on two-step photoionization of potassium atoms in a collimated differentially pumped beam; by tuning the ionizing wavelength from 455.2 to $424 \mathrm{~nm}$, the electron energy is continuously varied from zero to $200 \mathrm{meV}$. Typically, electron currents in the range $20-50 \mathrm{pA}$ are used to limit the energy broadening effects associated with the photoion space charge (FWHM about $30 \mu \mathrm{eV} / \mathrm{pA}$ ) (Bömmels et al., 2001). A differentially pumped supersonic beam serves as a well collimated target, allowing - in conjunction with the increased current - for the first time electron attachment studies of molecular clusters at meV energy width (Weber et al., 1999a). Negative ions which are created by electron attachment and drift out of the essentially field free reaction chamber, are imaged into a quadrupole mass spectrometer $(m / q \leq 2000 \mathrm{u} / \mathrm{e})$ with a combination of two electrostatic lenses. The transmitted ions are accelerated to an energy of $1 \mathrm{keV}$ and detected by a differentially pumped off-axis channel electron multiplier (Sjuts) with low background $\left(<0.02 \mathrm{~s}^{-1}\right)$. For diagnostics of the target beam (especially with respect to possible cluster formation), positive ion mass spectra can be generated by electron impact ionization with an auxiliary electron gun (current around $0.1-1 \mu \mathrm{A}$, energy $75-85 \mathrm{eV}$ ).

The reaction volume is surrounded by a cubic chamber, made of six insulated copper plates. To improve the homogeneity of the surface potentials, the inner walls are coated with colloidal graphite. By applying bias potentials to each plate, residual dc electric fields are reduced to values $F_{\mathrm{S}}<0.2 \mathrm{~V} \mathrm{~m}^{-1}$; the reduction procedure involves an iterative optimization of the shape of the attachment spectrum due to $\mathrm{SF}_{6}^{-}$formation around zero electron energy (Klar et al., 1994a; Schramm et al., 1998). Magnetic fields are reduced to values below $2 \mu \mathrm{T}$ by compensation coils located outside the vacuum apparatus (Klar et al., 1994a; Weber et al., 1999b) or by the use of reaction chambers fabricated of mu metal (Schramm et al., 1998, 1999, 2002). The electron energy resolution is limited by the bandwidth of the ionizing laser (normally $\Delta E_{\mathrm{L}} \approx 0.15 \mathrm{meV}$ ), residual electric fields $\left(\Delta E_{\mathrm{F}} \leq 0.3 \mathrm{meV}\right)$, the Doppler effect (present in both the photoionization and in the attachment process, $\Delta E_{\mathrm{D}} \approx 0.06 E^{1 / 2}$ for target velocities similar to the potassium atom velocity $\left(600 \mathrm{~ms}^{-1}\right)$ with $\Delta E_{\mathrm{D}}$ and $E$ in $\left.\mathrm{meV}\right)$, and 
space charge broadening $\Delta E_{\mathrm{SC}}$ due to $\mathrm{K}^{+}$photoions generated in the reaction volume $\left(\Delta E_{\mathrm{SC}} \approx 0.9 \mathrm{meV}\right.$ at $30 \mathrm{pA}$ photocurrent, see Fig. 7 in Bömmels et al., 2001). For the sake of normalization, in situ resolution testing, and compensation of electric stray fields, measurements of $\mathrm{SF}_{6}^{-}$ formation are carried out, using a seeded supersonic beam of about $0.05 \%$ $\mathrm{SF}_{6}$ in $\mathrm{He}\left(p_{0}=1 \mathrm{bar}, T_{0}=300 \mathrm{~K}\right)$. By comparison of the measured anion yield with the known cross-section for $\mathrm{SF}_{6}^{-}$formation (Klar et al., 1992a, b, 1994a; Schramm et al., 1998) near $0 \mathrm{eV}$ (convoluted with adjustable resolution functions), the effective electron energy spread at low energies can be inferred.

The use of a supersonic beam target has the substantial advantage of a spatially confined reaction volume. When the molecules of interest are seeded in light carrier gas (such as helium) the kinetic energy of the molecules is raised substantially above its thermal value which - under conditions of weak extraction fields - results in a higher detection efficiency of the product ions due to dissociative attachment (Barsotti et al., 2002b). In a mixed supersonic beam, containing the seeded minority component with molecular mass $m_{S}$ at a fraction $x$ and the atomic carrier gas with mass $m_{C}$ (fraction $1-x$ ), the flow velocity $u_{S}$ of the seeded component can be estimated (in the absence of velocity slip) by (Miller, 1988)

$$
u_{S} \approx\left\{5 \mathrm{k}_{\mathrm{B}} T_{0} /\left[x m_{S}+(1-x) m_{C}\right]\right\}^{1 / 2}
$$

with $\mathrm{k}_{\mathrm{B}}=$ Boltzmann constant.

A nontrivial aspect of DA experiments is a possible influence associated with the angular distribution of dissociating anions with respect to the momentum vector of the electron (Massey, 1976), as shown, e.g., for DA to $\mathrm{Cl}_{2}$ by Azria et al. (1982). This problem is relevant in DA experiments with a well-defined direction of the electron beam when resonances with different symmetries are involved, leading to different anion detection efficiencies, as long as the anions are detected in an angle-sensitive manner. In the TPSA and LPA experiments angular distribution effects are expected to be negligible (or small) because the photoelectrons, created in the center of the reaction region, are emitted in all directions (albeit not fully isotropically).

The LPA experiment provides highly resolved attachment yield spectra $Y_{\mathrm{e}}(E)$ for anion formation over the typical energy range $0.1-200 \mathrm{meV}$. The yield is proportional to the absolute cross-section $\sigma_{\mathrm{e}}(E)=N Y_{\mathrm{e}}(E)$ for anion formation due to free electron attachment. The normalization constant $N$ is conveniently determined with reference to known thermal energy attachment rate coefficients $k_{\mathrm{e}}(T)$ (Smith and Španel, 1994; 
Christophorou, 1996) using the expression (Chutjian and Alajajian, 1985; Klar et al., 1992b, 2001a, b; Chutjian et al., 1996; Schramm et al., 1999, 2002)

$$
k_{\mathrm{e}}(T)=N\left(2 / m_{\mathrm{e}}\right)^{1 / 2} \int_{0}^{\infty} Y_{\mathrm{e}}(E) E^{1 / 2} f(E ; T) \mathrm{d} E
$$

with the Maxwellian distribution function $f(E ; T)=(4 / \pi)^{1 / 2}\left(\mathrm{k}_{\mathrm{B}} T\right)^{-3 / 2} E^{1 / 2}$ $\exp \left[-E /\left(\mathrm{k}_{\mathrm{B}} T\right)\right]$ where $\mathrm{k}_{\mathrm{B}} T=25.85 \mathrm{meV}$ for $T=300 \mathrm{~K}$ and $\int_{0}^{\infty} f(E ; T) \mathrm{d} E=1$. In the rate coefficient $k_{\mathrm{e}}(T)$ the temperature $T$ addresses both the electron temperature $T_{\mathrm{e}}$ and the gas temperature $T_{\mathrm{G}}$ which are assumed to be identical. In the TPSA and the LPA experiments the gas temperature was typically $T_{\mathrm{G}}=300 \mathrm{~K}$; thus, rate coefficients $k_{\mathrm{e}}(T)$ obtained at $T=T_{\mathrm{e}}=$ $T_{\mathrm{G}}=300 \mathrm{~K}$ were used for normalization. As shown by Klar et al. $(1992 \mathrm{~b}$, 2001a) for the cases of $\mathrm{SF}_{6}$ and $\mathrm{CCl}_{4}$ (s-wave attachment, peaking at zero energy) an integration interval $(0,170) \mathrm{meV}$ in $(35)$ is sufficient to guarantee errors below $1 \%$ in the normalization. In the evaluation the near-zero energy range requires some care. Klar et al. (1992b, 2001a, b) and Schramm et al. $(1999,2002)$ used an analytical cross-section similar to the Klots crosssection (21) to extrapolate to zero energy (see Section IV.B). It has to be stressed that the normalization procedure (35) can only be carried out in a reliable way if the anion yield function is obtained at sufficiently narrow electron energy width and down to sufficiently low energies. We also emphasize that the gas temperature is an important parameter since attachment cross-sections may depend very strongly on the rovibrational distribution of the molecules (O'Malley, 1967; Chantry, 1969; Massey, 1976; Christophorou, 1987; Smith and Španel, 1994; Hahndorf and Illenberger, 1997).

We conclude this section by briefly mentioning another approach to study electron-molecule collisions at very low energies. Going back to ideas of Fermi (1934), electrons in Rydberg orbits can be used as a source of very slow electrons (Stebbings and Dunning, 1983; Klar et al., 1994b; Dunning, 1995). The concept of the quasi-free electron model (Fermi, 1934; Matsuzawa, 1972) allows us to express the rate coefficient $k_{n l}$ for a particular process to occur with Rydberg electrons in specified orbits $n l$ through the cross-section $\sigma_{\mathrm{e}}(\mathrm{v})$ for the same process involving free electrons (Matsuzawa, 1972):

$$
k_{n l}=\int_{0}^{\infty} \sigma_{\mathrm{e}}(\mathrm{v}) \mathrm{v} f_{n l}(\mathrm{v}) \mathrm{dv}
$$

where $f_{n l}(\mathrm{v})$ represents the velocity distribution function of the highly excited $n l$ electron in the Rydberg atom $\mathrm{A}^{* *}(n l)$. This equation assumes that the interaction of the electron with the ion core $\mathrm{A}^{+}$and that of the ion core $\mathrm{A}^{+}$ 
with the target system is negligible throughout the collision process. At sufficiently low $n$ this assumption is no longer valid (Dunning, 1995; Desfrançois et al., 1996; Compton and Hammer, 2001). Free electron crosssections $\sigma_{\mathrm{e}}(\mathrm{v})$ at very low velocities may be tested by comparing measured rate coefficients $k_{n l}$ with those calculated through Eq. (36) on the basis of the quasi-free electron model. The inverse procedure of deriving free electron cross-sections from a measured $n$ dependence for a RET process is not unique and so far has not been demonstrated in a convincing way.

\section{Case Studies}

In the following section we shall discuss in some detail recent results which highlight resonance and threshold effects in low-energy electron collisions with selected molecules and molecular clusters, as obtained at very high resolution (i.e. energy widths in the few $\mathrm{meV}$ range). The emphasis will be on angle-differential elastic and vibrationally inelastic electron scattering as well as on electron attachment studies, but examples for total scattering cross-sections will also be included. Moreover, we shall briefly discuss important recent observations made for annihilation of positrons traversing molecular gases at energies below $1 \mathrm{eV}$ at sufficiently low energy widths to resolve vibrational structure. Since the long-range electron-molecule interaction plays a decisive role at the considered low collision energies the molecules are grouped accordingly.

\section{A. Electron Collisions with Polar Molecules}

Most molecules in nature are polar species. The presence of a permanent dipole moment has strong effects on electron-molecule scattering at low energies since the electron-dipole interaction and the centrifugal potential have to be treated on an equal footing as pointed out in the theory section. In Section IV.A we first discuss two groups of such molecular species (the hydrogen and methyl halides) which exhibit a rich spectrum of resonance and threshold effects and which have played an important role in the development of the theory for electron-molecule collisions. In addition we discuss as a special case of interest the molecule $1,2-\mathrm{C}_{2} \mathrm{H}_{2} \mathrm{~F}_{2}$ : a comparative investigation of elastic and inelastic electron scattering has been carried for both the cis-form (dipole moment $\mu=2.42 \mathrm{D})$ and the trans-form $(\mu=0)$.

\section{A.1. Hydrogen Halides}

Studies of threshold behavior in electron collisions with hydrogen halides $\mathrm{H} X$ (where $X$ stands for $\mathrm{F}, \mathrm{Cl}, \mathrm{Br}$ and I atoms) started with the pioneering 
work of Rohr and Linder $(1975,1976)$ and $\operatorname{Rohr}(1977,1978)$ who discovered threshold peaks in $\mathrm{VE}$ of $\mathrm{HF}, \mathrm{HCl}$, and $\mathrm{HBr}$ molecules. Threshold structures were also found in the elastic cross-section (Burrow 1974) and in transmission spectra (Ziesel et al., 1975a).

It was realized later that these peaks are related to the stepwise structure in the DA cross-section at VE thresholds, observed first by Ziesel et al. (1975a) for $\mathrm{HCl}$ and later by Abouaf and Teillet-Billy $(1977,1980)$ for $\mathrm{HCl}$, $\mathrm{DCl}, \mathrm{HBr}$ and $\mathrm{HF}$ molecules and explained by nonlocal effects as early as in 1974 (Fiquet-Fayard, 1974). Another distinctive property of DA crosssections to hydrogen halides is a vertical threshold onset which is a general feature of an endothermic DA process without a reaction barrier (O'Malley, 1966). More detailed experimental data on VE were obtained in the late 1980s in Ehrhardt's group (Knoth et al., 1989a,b; Rädle et al., 1989). In the $0 \rightarrow 3 \mathrm{VE}$ cross-section for HF, they found a dip-like resonance structure below the $v=4$ threshold (Knoth et al., 1989a), thus providing the first experimental evidence for a vibrational Feshbach resonance (VFR, then addressed as nuclear-excited resonance). Recently, the Fribourg group reported improved results for VE of all the hydrogen halide molecules (Allan et al., 2000, Sergenton et al., 2000, Sergenton and Allan, 2000; Čížek et al., 2001a; Allan, 2001b), as will be in part discussed below.

Initial attempts to explain threshold peaks in VE were based on model calculations involving enhancement by a virtual state (Dubé and Herzenberg, 1977; Kazansky, 1978, 1982), s-wave bound states (Gauyacq, 1983; Teillet-Billy and Gauyacq, 1984), or the long-range dipolar interaction between the electron and the target (Fabrikant, 1977, 1983). (see Morrison, 1988 for a more complete review of the work done before 1988). An important paper of Domcke and Mündel (1985), based on the nonlocal complex potential approach (Domcke, 1991), showed that the situation is much more complex. In the nonlocal treatment the effects of resonances, virtual states and bound states are all included and distinction between them is not easily possible. The long range dipolar interaction between the electron and the molecule leads to a very strong energy dependence of the resonance capture amplitudes and an unusual behavior of an s-wave resonance in an adiabatic fixed-nuclei approximation.

In the early 1990s a series of model and semiempirical calculations of DA and VE for hydrogen halides were performed which led to an understanding of the basic mechanisms. Some of these calculations were based on the quasiclassical version of the nonlocal complex potential theory (Kalin and Kazansky, 1990), and some on the quasiclassical version of the resonance $R$-matrix theory (Fabrikant, 1991a) which was shown (Fabrikant, 1990) to be equivalent to the nonlocal approach. For model or semiempirical calculations the $R$-matrix version is more convenient since it allows for 
a simpler parameterization of the input parameters. Quasiclassical $R$-matrix calculations for $\mathrm{HCl}$ and $\mathrm{HF}$ (Fabrikant, 1991a; Fabrikant et al., 1991, 1992) reproduced the major features in VE and DA cross-sections, showing essential agreement with earlier calculations (Domcke and Mündel, 1985) for $\mathrm{HCl}$. Variational $R$-matrix calculations (Thümmel et al., 1992, 1993) obtained individual rotational contributions to vibrational excitation of HF, but did not produce results for DA. The effective range approximation was applied by Gauyacq (1987).

Further development of theoretical methods allowed obtaining accurate ab initio cross-sections for HF (Gallup et al., 1998), HCl (Horáček et al., 1998; Čížek et al., 1999; Allan et al., 2000), HBr and DBr (Horáček and Domcke, 1996, Ćížek et al., 2001), and HI (Horáček et al., 1997; Kolorenc et al., 2002). Near-threshold DA to vibrationally and rotationally excited molecules has been studied by Xu et al. (2000) for HF and Kolorenc et al. (2002) for HI.

Improved experimental techniques led to the discovery of unexpected oscillatory structures in $\mathrm{VE}$ of $\mathrm{HCl}$ below the DA threshold (Schafer and Allan, 1991; Cvejanović 1993). Figure 16 shows the more detailed
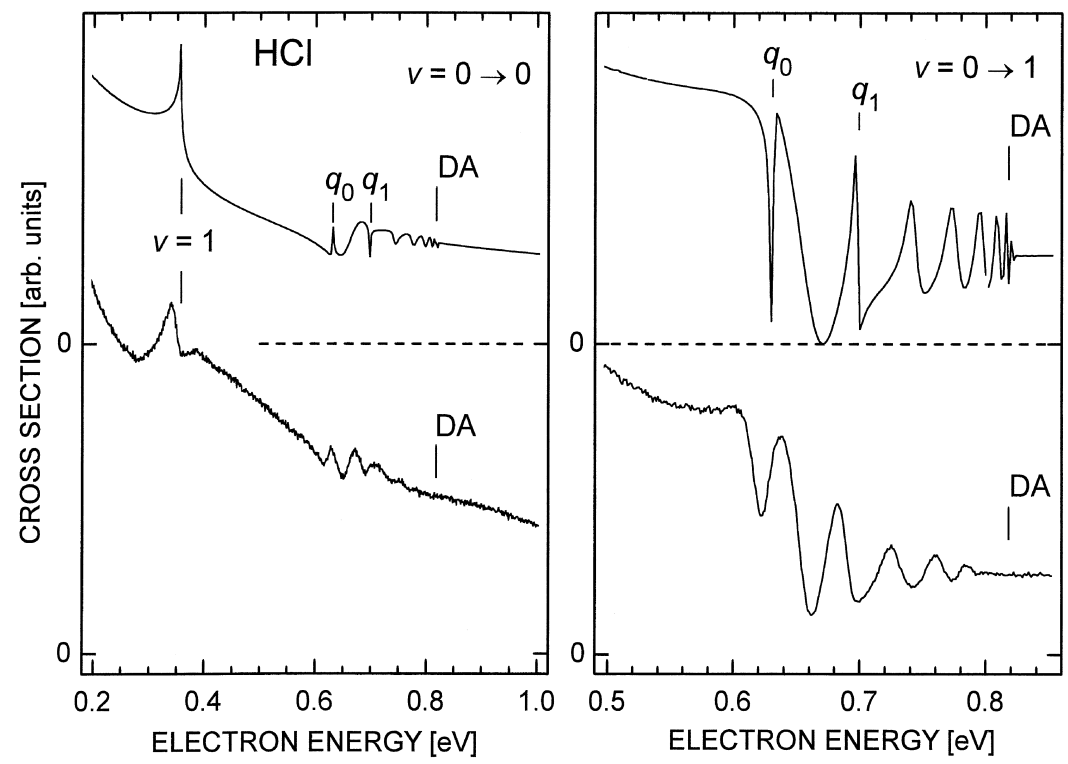

Fig. 16. Elastic (left) and $v=0 \rightarrow 1 \mathrm{VE}$ cross-sections measured at $90^{\circ}$ in $\mathrm{HCl}$. Results of nonlocal resonance theory are shown on the top, experiment on the bottom. The sharp structures due to outer-well resonances $\left(q_{0}\right.$ and $\left.q_{1}\right)$ are superimposed on broader boomerang type oscillations (Allan et al., 2000). 


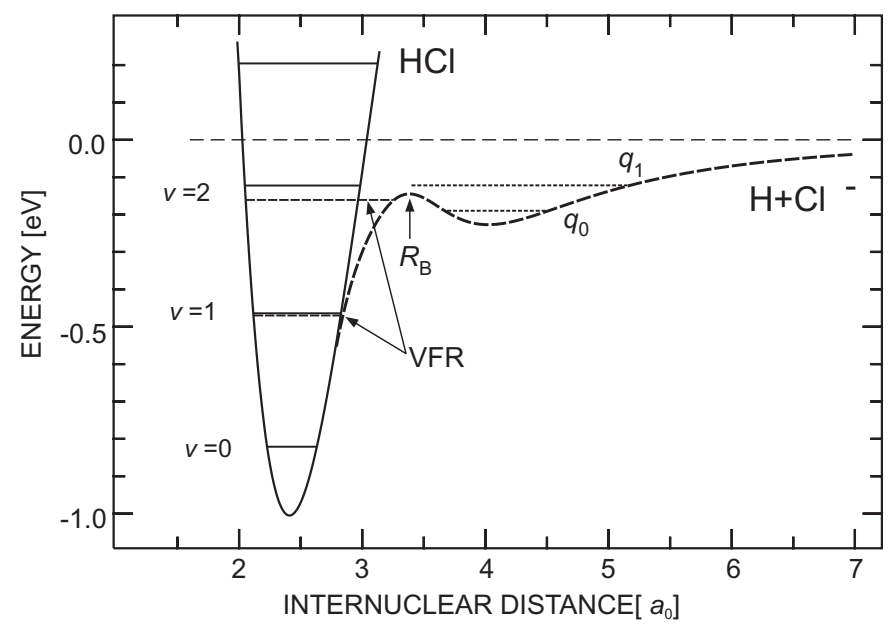

FIG. 17. Illustration of the outer- and inner-well resonances in $\mathrm{HCl}$. The adiabatic potential curves of Č́žek et al. $(1999,2001 \mathrm{~b})$ are shown as solid $(\mathrm{HCl})$ and dashed $\left(\mathrm{HCl}^{-}\right)$curves (see also text).

measurements and results of advanced nonlocal resonance theory calculations of Allan et al. (2000). The theory (Č́žzek et al., 1999; Allan et al., 2000) permitted an interpretation of these structures as a combination of boomerang oscillations, reflecting short-lived wave-packet motion of the $\mathrm{HX}^{-}$anion, and so-called outer-well resonances, arising from quasibound energy levels in the outer well of the anion potential curve, as illustrated in Fig. 17. The $\mathrm{HCl}^{-}$curve follows the general model discussed in Sections I and II, Figs. 3 and 6 . It has an inner and an outer well, separated by a potential barrier at $R_{B}$. The adiabatic potential curve of $\mathrm{HCl}^{-}$disappears below $R \approx 2.8 a_{0}$, but vibrational motion of short duration is still possible on the inner well through nonadiabatic effects (partial re-capture of the electronic cloud as the nuclei swing back to large $R$ ), giving rise to the VFRs. Quasistationary vibrational levels whose wave function is localized predominantly in the outer well give rise to the outer-well resonances. They are coupled to the inner well by tunneling $\left(q_{0}\right)$ or passage over the potential barrier $\left(q_{1}\right)$. The outer well resonances appear as sharp dips at 0.632 and $0.699 \mathrm{eV}$, the VFR associated with the $v=2$ level as a dip at $0.66 \mathrm{eV}$ in the spectra of Fig. 16. Note the striking similarity with the situation encountered in $\mathrm{CO}_{2}$ (Fig. 34). The outer-well resonances may be viewed as vibrational Feshbach resonances, albeit somewhat different in a quantitative sense from the 'inner well vibrational Feshbach resonances' discussed in the introduction. Similar structures as for $\mathrm{HCl}$ were observed in the elastic 
and VE cross-sections of $\mathrm{HBr}$ and $\mathrm{DBr}$ by Č́ižek et al. (2001a), who also presented improved calculations and measurements of the dissociative attachment channel.

For HF, oscillatory structure below the DA threshold starts to be observed in the $v=0 \rightarrow 3 \mathrm{VE}$ channel and becomes very clear in $v=0 \rightarrow 4$ VE (Sergenton et al., 2000). Recent high-resolution (15 meV) angledifferential VE results (Allan, 2001b; Ćížek et al., 2003) for $v=0 \rightarrow 1,2,3$, 4 exhibit a plethora of threshold and resonance features which are in full harmony with a nonlocal resonance model calculation of Ćížek et al. (2003): in the $v=2$ channel, a sharp, dip-like VFR just below the $v=3$ onset is found (as predicted by Thümmel et al., 1993); in the $v=3$ channel, a broader, deep dip below the $v=4$ threshold is observed (confirming and improving the results of Knoth et al., 1989a), followed by oscillatory structure; in the $v=4$ channel a weak threshold peak is followed by impressive oscillations with decreasing energy spacings which cease at the DA threshold. These results are presented in Fig. 18a and compared with results of a nonlocal resonance model calculation (Čížek et al., 2003).

Impressive agreement between theory and experiment is observed. The structures can be understood as VFR, which are sharp at lower energies
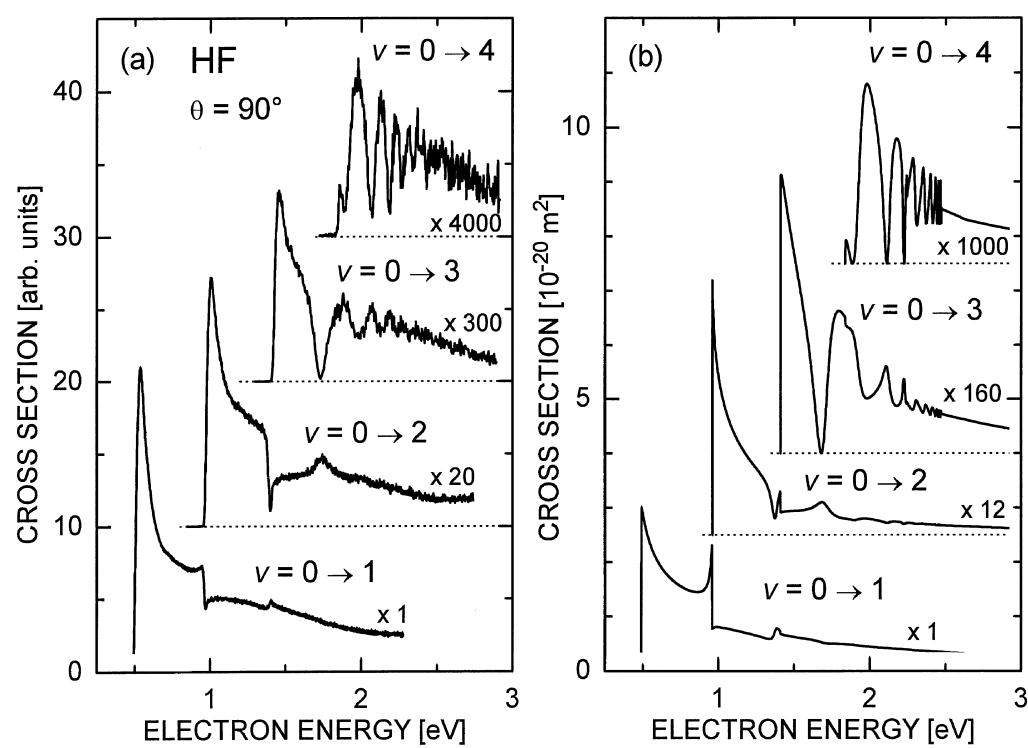

Fig. 18. VE cross-sections for $\operatorname{HF}(v=0)$. (a): experiment $\left(\theta=90^{\circ}\right.$, Allan, 2001b; Ćížek et al., 2003) (b) theory (Čížek et al., 2003). 
and gradually become boomerang oscillations at higher energies, where the anion lifetime is just enough for nuclei to perform one classical oscillation. This interpretation might at first seem to cause a problem because $\mathrm{H} X$ molecules do not support bound states at relatively small internuclear distances, close to the equilibrium separation and below (the same situation occurs for methyl halides discussed in Section IV.A.2). Appropriate theories predict VFRs even in this case, however (Section II). The qualitative picture (Gauyacq, private communication, see also Sergenton et al., 2000) is that the electron escapes at small internuclear separations so slowly that it is recaptured with a large probability when the nuclei swing back to large $R$ where it is bound. Another interesting feature of boomerang oscillations is that they always occur below the DA threshold. Strictly speaking, boomerang structure could also occur above the DA threshold in the presence of potential barriers towards dissociation from which the nuclear wave packet would be reflected. No such case of boomerang structure above DA threshold has yet been reported, however. The same is true for threshold peaks in VE which are generally found only below the DA threshold (Domcke, 1991, Ćižek et al., 2001a), but may appear above it in the presence of a potential barrier towards dissociation. A specific example of the latter case was recently found, first theoretically (Schramm et al., 1999) and then experimentally (Allan and Fabrikant, 2002), in methyl iodide. The fact that the DA channel is open in the VFR region simply means that there is an additional channel for the VFR decay: predissociation into the valence ionic state. Indeed, as will be discussed in Section IV.A.2, VFRs also appear in the DA channel in electron collisions with methyl halides. This mechanism is similar to what is called "indirect recombination" (O'Malley, 1981) in the theory of dissociative recombination and was discussed a lot in the relevant literature (Mitchell, 1990).

\section{A.2. Methyl Halides and Related Molecules}

Dissociative attachment (DA) in low-energy electron collisions with methyl halide molecules $\mathrm{CH}_{3} X(X=\mathrm{F}, \mathrm{Cl}, \mathrm{Br}, \mathrm{I})$, yielding $X^{-}$anions, as well as vibrational excitation (VE) of the $\mathrm{C}-X$ stretch mode $\nu_{3}$ may be described by a one-dimensional model involving just the $v_{3}$ mode. Although DA at zero electron energy is an exothermic process for the heavier methyl halides $(X=\mathrm{Cl}, \mathrm{Br}, \mathrm{I})$, the DA rate coefficients differ by orders of magnitudes at room temperature, rising from an extremely small value for $\mathrm{CH}_{3} \mathrm{Cl}$ (of order $10^{-15} \mathrm{~cm}^{3} \mathrm{~s}^{-1}$ ) via about $10^{-11} \mathrm{~cm}^{3} \mathrm{~s}^{-1}$ for $\mathrm{CH}_{3} \mathrm{Br}$ to about $10^{-7} \mathrm{~cm}^{3} \mathrm{~s}^{-1}$ for $\mathrm{CH}_{3} \mathrm{I}$ (Smith and Španel, 1994; Christophorou, 1996). A comparative study of DA to these molecules can help understand the basic physics governing the magnitudes of the DA cross-sections for different 
molecules and the dependence of the DA cross-sections on vibrational temperature and electron energy.

DA to $\mathrm{CH}_{3} \mathrm{Cl}$ was investigated by swarm and beam techniques (for more recent studies and references to earlier work see Petrović et al., 1989; Chu and Burrow, 1990; Datskos et al., 1990; Pearl and Burrow, 1993). Widely different results for the DA cross-sections were reported that disagree by many orders of magnitude. Careful experimental investigations (Pearl and Burrow; 1993, Pearl et al., 1995), supported by semiempirical calculations (Fabrikant, 1991b, 1994; Pearl et al., 1995), showed that most of the earlier measurements were affected by contaminants, and the actual value of the low-energy DA cross section for $\mathrm{CH}_{3} \mathrm{Cl}$ gas at room temperature is so small that the process can hardly be detected. However, an increase in molecular temperature leads to a very rapid exponential rise of the cross-section. Theory and experiment agree well for temperatures above about $500 \mathrm{~K}$ (Pearl et al., 1995). DA cross-sections for $\mathrm{CH}_{3} \mathrm{Br}$ are substantially larger than for $\mathrm{CH}_{3} \mathrm{Cl}$, but still rather small at room temperature. At $T=300 \mathrm{~K}$ the swarm unfolded DA cross section of Datskos et al. (1992) shows a peak at $E \approx 0.38 \mathrm{eV}$ with a size of about $1.8 \times 10^{-22} \mathrm{~cm}^{2}$. Both the energy integrated cross section and the rate coefficient for electron attachment to $\mathrm{CH}_{3} \mathrm{Br}$ exhibit a strong increase with rising molecular temperature (Spence and Schulz, 1973; Alge et al., 1984; Petrović and Crompton, 1987; Datskos et al., 1992). So far high-resolution electron attachment spectra for $\mathrm{CH}_{3} \mathrm{Cl}$ and $\mathrm{CH}_{3} \mathrm{Br}$ have not been reported. DA to $\mathrm{CH}_{3} \mathrm{I}$ was studied experimentally by electron beam (Spence and Schulz, 1973) and swarm (Christophorou, 1976; Alge et al., 1984; Shimamori and Nakatani, 1988; Shimamori et al., 1992a; Speck et al., 2000) methods and by the threshold photoelectron attachment technique (Alajajian et al., 1988). In recent LPA studies of DA to the $\mathrm{CH}_{3} \mathrm{I}$ molecule (Hotop et al., 1995; Schramm et al., 1999), performed with $\mathrm{meV}$ resolution, a sharp variation of the cross section within a narrow energy interval below the first threshold for vibrational excitation of the symmetric stretch $\nu_{3}=1$ was observed. This was interpreted in terms of a vibrational Feshbach resonance (Schramm et al., 1999). Higher vibrational thresholds do not exhibit resonance behaviour, but show pronounced cusps of the Baz'-Wigner type (Baz' 1958).

Using the model outlined in II.A.4, $R$-matrix calculations were performed (Schramm et al., 1999; Wilde et al., 2000) with the aim to theoretically characterize vibrational structure in the DA cross-sections for the methyl halides and to understand the strong size variation and temperature dependences of the DA rates for $\mathrm{CH}_{3} \mathrm{Cl}$ and $\mathrm{CH}_{3} \mathrm{Br}$. The parameters of the neutral potential curve $V(\rho)$, described in Morse form, were chosen to reproduce the experimental values for the dissociation energy $D_{0}$ as well as the vibrational spacing $\Delta G_{0-1}\left(v_{3}\right)$. For the negative ion curve $U(\rho)$, 
described by an exponentially decaying function plus a constant, the vertical attachment energy (i.e. the value at $\rho=0$, the equilibrium distance of the neutral potential) was chosen to agree with estimates of Modelli et al. (1992) (obtained from electron transmission spectra). The asymptotic value of $U(\rho)$ is fixed by the combined value of $D_{0}\left(\mathrm{CH}_{3}-X\right)$ and the well-known electron affinity of $X(X=\mathrm{Cl}, \mathrm{Br}, \mathrm{I})$ (Andersen et al., 1999). This leaves as unknown quantities in the semiempirical $R$-matrix model the parameter $\beta$ of the exponential repulsion in $U(\rho)$ and the surface amplitude (related to the width function), described by $\gamma(\rho)=\gamma_{0}+\gamma_{1} /[\exp (\zeta \rho)+\eta]$. Moreover, the important long-range interactions between the electron and the molecule were taken into account in an appropriate way.

For methyl chloride, the $R$-matrix parameters were fixed by using information from $a b$ initio calculations for the anion curve at distances $\rho>\rho_{\mathrm{c}}\left(\rho_{\mathrm{c}}\right.$ denotes the crossing point between $V(\rho)$ and $\left.U(\rho)\right)$ and from features observed in measurements of the differential VE cross section for $\mathrm{CH}_{3} \mathrm{Cl}$. For methyl iodide, the parameters were obtained by fitting to the highly resolved absolute DA cross-section, obtained by Schramm et al. (1999) with reference to swarm data at $300 \mathrm{~K}$. For methyl bromide, two approaches were used which gave quite different results; we here report the results from model 2 which incorporated information from ab initio calculations for the anion curve as well as use of the experimental DA rate coefficient of Petrović and Crompton (1987) at $T=440 \mathrm{~K}$.

In Fig. 19 we show the relevant potential curves for the three methyl halides of interest. The most important characteristic and difference between the three molecules is the location of the crossing point between the potential curve of the neutral molecule and that of the diabatic anion state which is close to the outer classical turning point of the $v_{3}=8,5$, and 2 vibrational level for $\mathrm{CH}_{3} \mathrm{Cl}, \mathrm{CH}_{3} \mathrm{Br}$, and $\mathrm{CH}_{3} \mathrm{I}$, respectively.

In Fig. 20 we present DA cross-sections for two different initial vibrational states of $\mathrm{CH}_{3} \mathrm{Cl}$, namely for $v_{3}=0$ and for $v_{3}=7$; note that the cross-section scales differ by more than eight orders of magnitude. Just below the onset for vibrational excitation of the $\nu_{3}=8$ level, a very sharp resonance is observed which is interpreted as a VFR (Wilde et al., 2000). When excited from the $v_{3}=0$ initial level, this VFR occurs at an energy of $0.68 \mathrm{eV}$ (peak cross section about $3 \times 10^{-27} \mathrm{~m}^{2}$ ) whereas in the attachment spectrum for $\mathrm{CH}_{3} \mathrm{Cl}\left(v_{3}=7\right)$ the same VFR shows up at $0.085 \mathrm{eV}$ with a peak cross section of about $1.1 \times 10^{-18} \mathrm{~m}^{2}$. The prominent VFR is present in all $\mathrm{CH}_{3} \mathrm{Cl}\left(v_{3}\right)$ attachment spectra for initial levels $v_{3}=0-7$. Sharp downward steps (cusps) are observed at the onsets for $v_{3}>8$ while a small peak (indicative of a virtual state) is present at the $v_{3}=7$ onset in the spectra for $\mathrm{CH}_{3} \mathrm{Cl}\left(v_{3}=0-6\right)$. The adiabatic potential curve for the $\mathrm{CH}_{3} \mathrm{Cl}^{-}$anion follows the neutral curve in the region close to equilibrium internuclear 


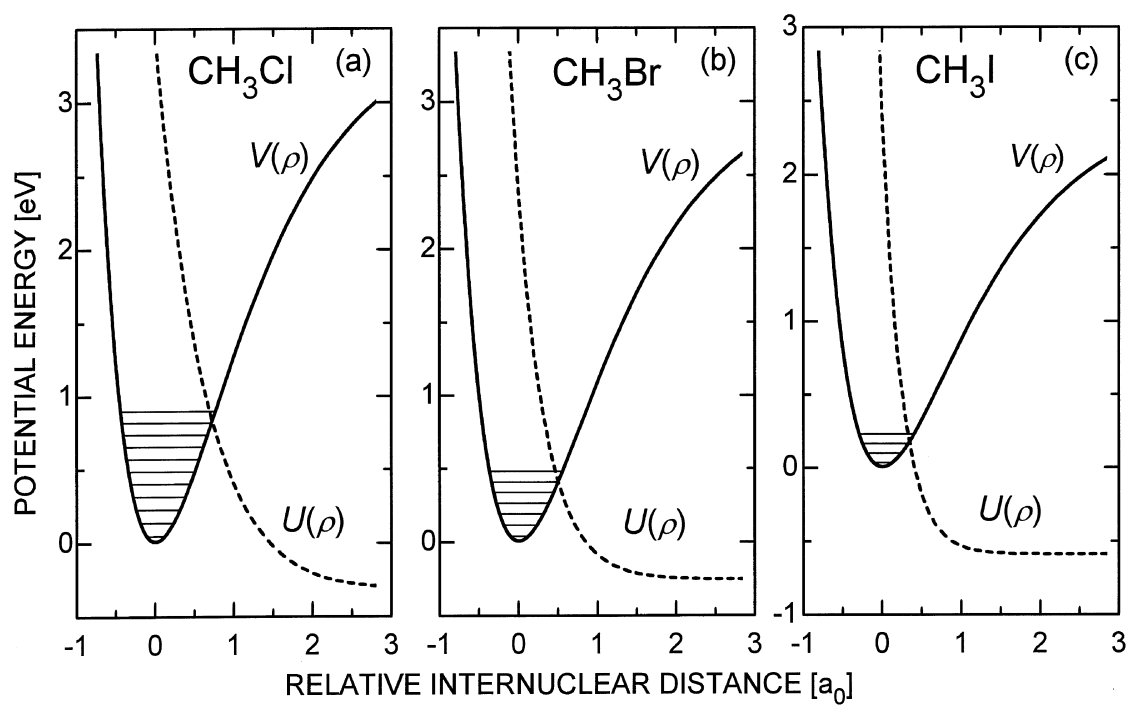

FIG. 19. Potential energy curves for $\mathrm{CH}_{3} \mathrm{Cl}$ (a) $\mathrm{CH}_{3} \mathrm{Br}$ (b) and $\mathrm{CH}_{3} \mathrm{I}$ (c) and their anions. The neutral curves (full) are denoted by $V(\rho)$, the diabatic anion curves (broken) by $U(\rho)$ where $\rho$ denotes the $\mathrm{C}-X$ distance relative to its equilibrium value $(\rho=0)$ (from Wilde et al., 2000).
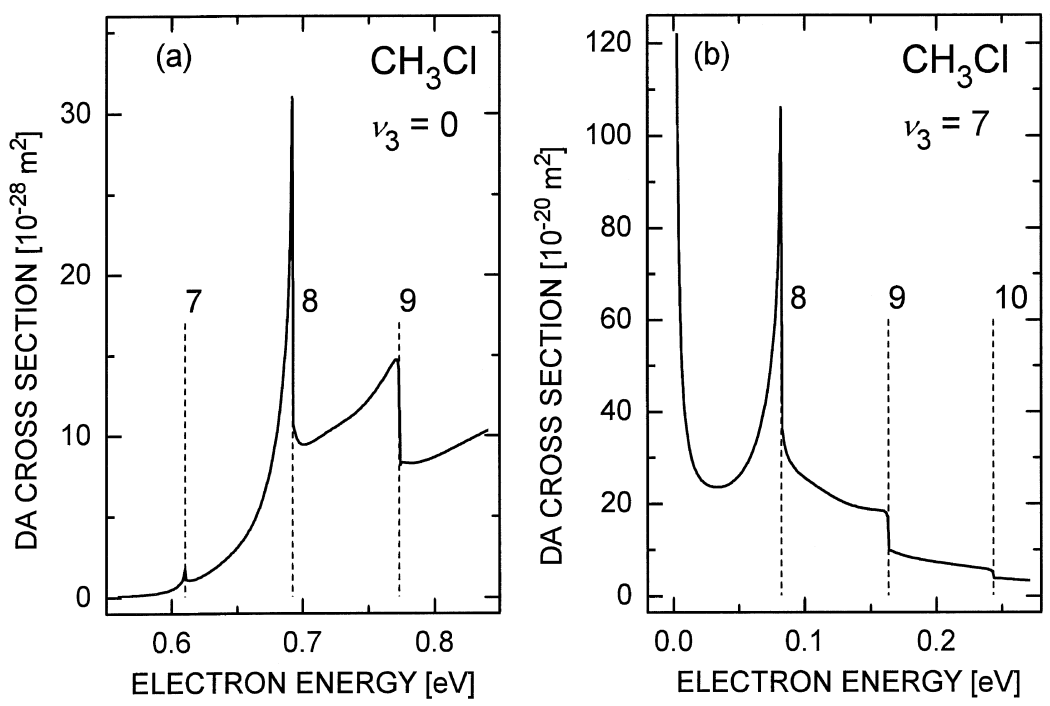

FIG. 20. Calculated DA cross-sections for $\mathrm{CH}_{3} \mathrm{Cl}$ ((a) $v_{3}=0$, (b) $v_{3}=7$ ). The quantum number $v_{3}$ labels the initially populated $\mathrm{C}-\mathrm{Cl}$ stretch vibrational mode. Vertical broken lines denote the indicated vibrational thresholds (from Wilde et al., 2000). 
separation (Wilde et al., 2000; see also the general case illustrated in Fig. 3b), producing about eight vibrationally excited states of $\mathrm{CH}_{3} \mathrm{Cl}^{-}$with a loosely bound electron, reflected as sharp peaks in the DA cross-sections (Fig. 20). In the case of $\mathrm{CH}_{3} \mathrm{I}$ we have only one vibrationally excited state of this type, therefore the resonance occurs only at the $v_{3}=1$ threshold. Although there are eight such states in $\mathrm{CH}_{3} \mathrm{Cl}^{-}$, the resonances at the thresholds with $\nu_{3}<7$ are masked by a rapid decrease of the DA cross-section towards lower energies due to the very fast drop of the Franck-Condon overlap between the initial vibrational state and the dissociating state.

A similar picture is observed for $\mathrm{CH}_{3} \mathrm{Br}$. Here the adiabatic negative-ion curve supports four excited vibrational states. Due to the lower a diabatic anion curve in the region of the crossing point, we observe two vibrational Feshbach resonances at the $v_{3}=3$ and $v_{3}=4$ thresholds (see Fig. 21; note that VFR at the $v_{3}=3$ threshold is barely seen on the scale of drawing). In the DA spectrum for $\mathrm{CH}_{3} \mathrm{Br}\left(v_{3}=0\right)$ the $v_{3}=4$ VFR has a peak cross-section of about $2 \times 10^{-22} \mathrm{~m}^{2}$ while in the spectrum for $\mathrm{CH}_{3} \operatorname{Br}\left(v_{3}=3\right)$ it reaches $1.2 \times 10^{-18} \mathrm{~m}^{2}$. The attachment spectrum for $\mathrm{CH}_{3} \mathrm{Br}$ molecules at room temperature is predicted to exhibit the $v_{3}=4 \mathrm{VFR}$ at four different energies in about equal strength (effective peak cross-sections around $3 \times 10^{-22} \mathrm{~m}^{2}$ ), corresponding to excitation from the thermally populated $v_{3}=0,1,2$, and 3
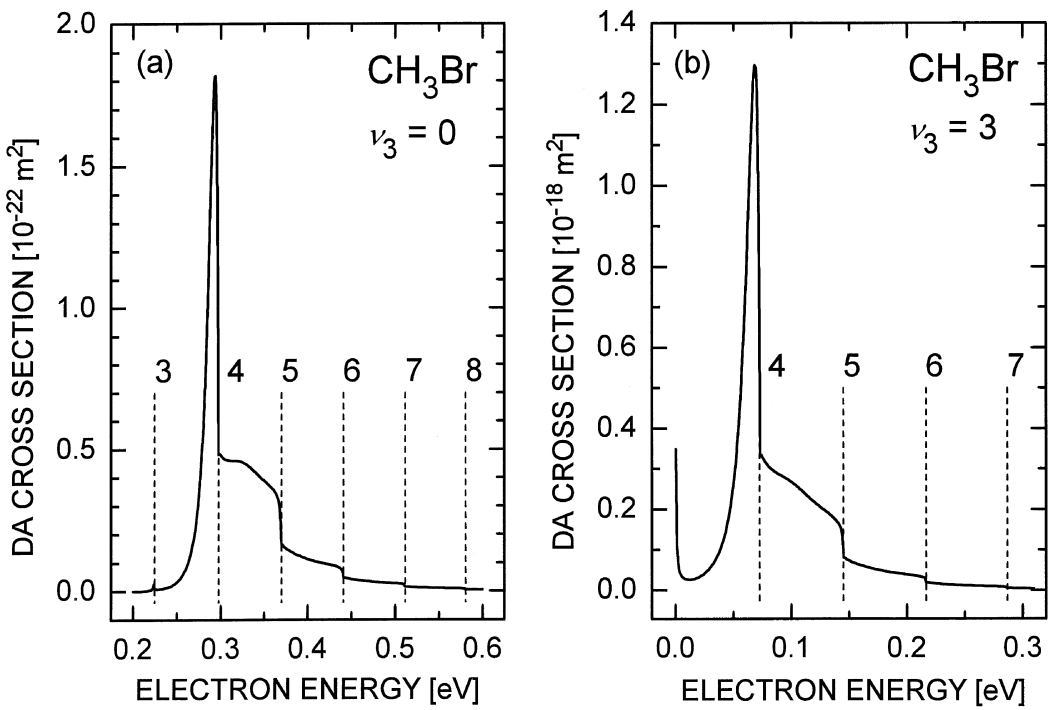

FIG. 21. Calculated DA cross-sections for $\mathrm{CH}_{3} \mathrm{Br}\left((\mathrm{a}) v_{3}=0\right.$, (b) $\left.v_{3}=3\right)$. Vertical broken lines denote the indicated vibrational thresholds (from Wilde et al., 2000). 
vibrational levels (Wilde et al., 2000). As mentioned above, two different models with more or less equal diabatic curves, but rather different surface amplitudes were used which yielded rather different cross-sections. The results shown here were obtained with the higher surface amplitude (model 2). High-resolution DA experiments for methyl bromide are needed to prove the presence of the prominent VFR associated with the $v_{3}=4$ level.

The first clear experimental observation of a VFR in the DA channel was made for $\mathrm{CH}_{3} \mathrm{I}$ by the Kaiserslautern group (Hotop et al., 1995; Schramm et al., 1999). Results were obtained with a thermal target $(300 \mathrm{~K}$, population of $v_{3}=1$ relative to $v_{3}=0$ about $7.8 \%$ ) as well as with vibrationally cooled molecules in a seeded supersonic beam. In Fig. 22 the supersonic beam data (open circles, adjusted in absolute size to theory) are compared with the $R$-matrix fit over the range $0-115 \mathrm{meV}$. Note that at the $v_{3}=2$ threshold VFR peak structure is absent in agreement with the experimental data, taken over a broader electron energy range at $T_{\mathrm{G}}=300 \mathrm{~K}$ (insert in Fig. 22; note that the experimental cross-section has been multiplied by 0.5 for this comparison). Weak structure is observed in the measured spectrum close

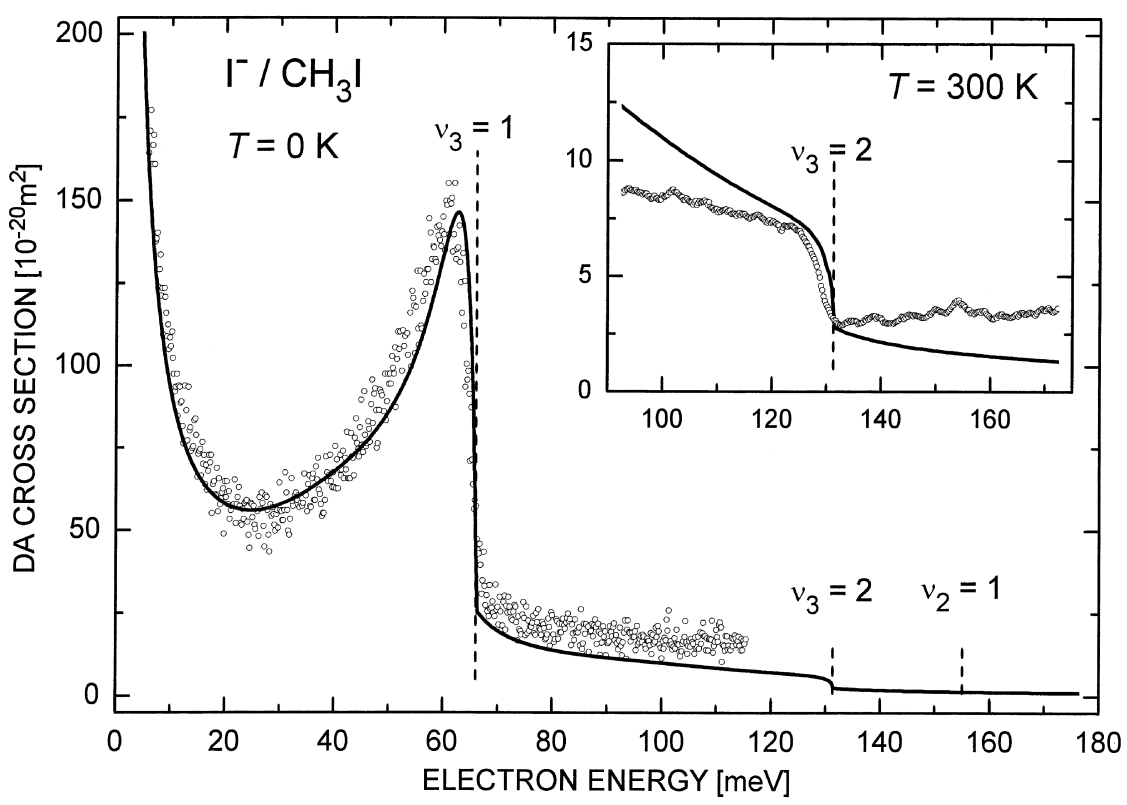

FIG. 22. Comparison of measured (open circles) and calculated (full curves) DA crosssections for $\mathrm{CH}_{3} \mathrm{I}$. The LPA data in the main frame were measured with a supersonic beam target, whereas for the LPA data in the inset (measured cross-section multiplied by 0.5 ) a diffuse gas target at room temperature was used (from Schramm et al., 1999). 
to the $v_{2}=1$ onset $(E=155 \mathrm{meV})$ which is not reflected by theory because only the $v_{3}$ mode is included in the model. The $R$-matrix results for the shape of the VFR were found to be very sensitive to the $R$-matrix parameters including the long-range electron-molecule interaction, associated with the permanent electric dipole moment and the polarizability of methyl iodide (Schramm et al., 1999; Leber et al., 2000a, see also Section II).

In view of the good quality in the description of both the shape and the absolute value of the DA cross-section one may hope that the $R$-matrix model be able to correctly predict electron scattering cross-sections, especially for VE involving the $v_{3}$ mode. In Fig. 23 we compare the calculated cross-sections for elastic and vibrationally inelastic $\left(v_{3}=0 \rightarrow 1\right)$ scattering with recent experimental results, with an energy width of $10 \mathrm{meV}$ in the incident beam (Allan and Fabrikant, 2002). In the elastic channel the VFR just below the $v_{3}=1$ onset shows up as a sharp dip whose depth is reduced in the experimental spectrum due to the energy spread. The $v_{3}=0 \rightarrow 1 \mathrm{VE}$ spectrum exhibits a prominent threshold peak, an upward step at the $v_{3}=2$ threshold, and an upward cusp at the $v_{3}=3$ onset in very good agreement between theory and experiment. Similarly good agreement is observed for $v_{3}=0 \rightarrow 2$ and higher channels VE. We conclude that semiempirical $R$-matrix calculations have substantial predictive power once the model parameters have been appropriately fixed by using information from either VE or DA experiments in combination with ab initio molecular structure theory.

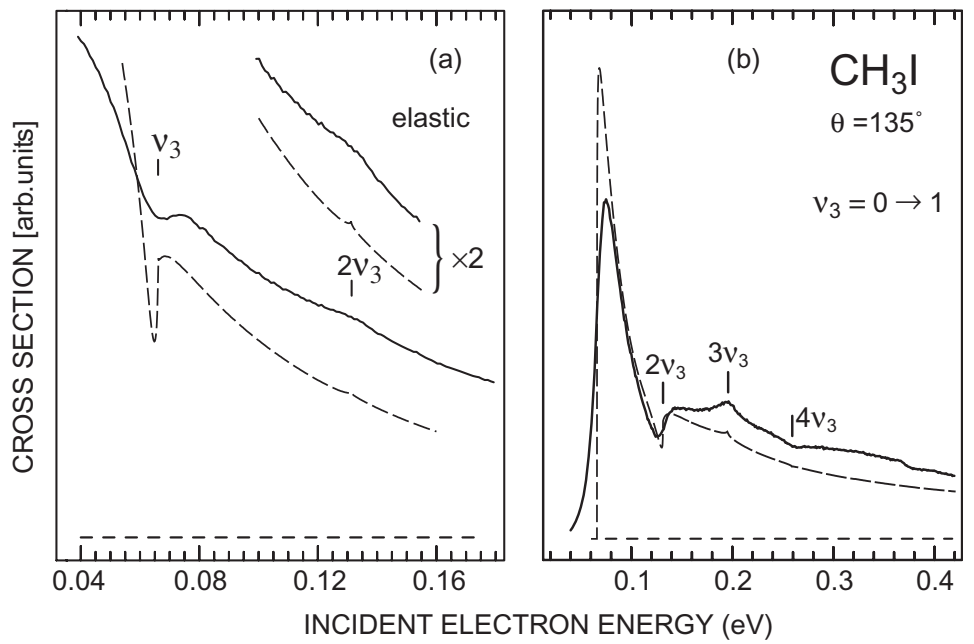

FIG. 23. Theoretical (dashed) and experimental elastic scattering (a) and $v_{3}=0 \rightarrow 1 \mathrm{VE}$ (b) in electron- $\mathrm{CH}_{3} \mathrm{I}$ collisions. Vibrational thresholds are marked (from Allan and Fabrikant, 2002). 


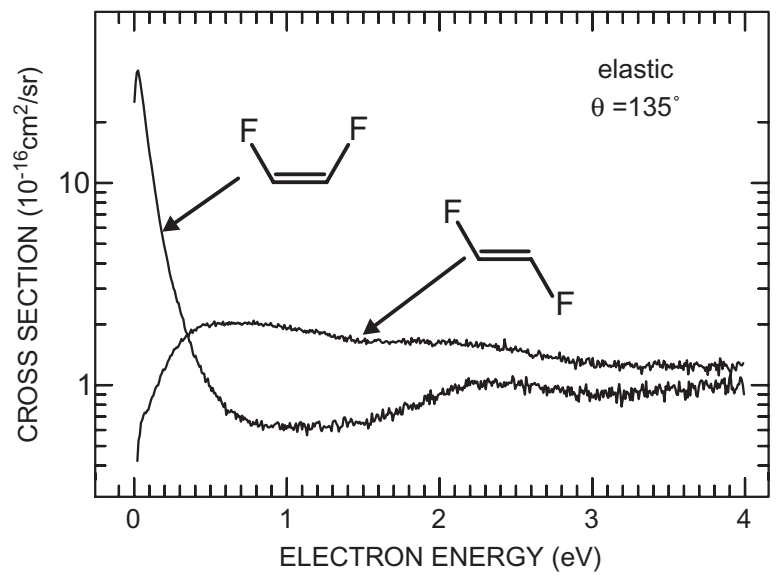

FIG. 24. Differential elastic cross-sections in cis- and trans-difluoroethenes (Allan et al., 2002).

A similar treatment can be applied to the perfluoromethyl chloride $\left(\mathrm{CF}_{3} \mathrm{Cl}\right)$ molecule. However, since its dipole moment is relatively small $(\mu=0.196$ a.u.), no VFRs were found there. A strong temperature effect in DA to $\mathrm{CF}_{3} \mathrm{Cl}$ was detected by Hahndorf et al. (1994) in the low-energy region (energy width around $0.1 \mathrm{eV}$ ). Theoretical calculations have been performed in the classical approximation (Lehr and Miller 1996; Lehr et al., 1997 ) and by the use of the resonance $R$-matrix method (Wilde et al., 1999). Recent ab initio calculations of Beyer et al. (2001) combine the $R$-matrix method with the projection operator technique to treat the vibrational dynamics. The semiempirical classical and the $R$-matrix calculations reproduce (albeit only qualitatively) the experimental zero-energy peak at $T=800 \mathrm{~K}$. For a more detailed comparison between theory and experiment, high resolution DA measurements as a function of gas temperature would be interesting.

The LPA method has been applied to investigate DA to the dipolar halogenated methanes $\mathrm{CH}_{3} \mathrm{I}$ (see above), $\mathrm{CFCl}_{3}, \mathrm{CBrCl}_{3}$, and $\mathrm{CH}_{2} \mathrm{Br}_{2}$ at meV energy width. In all cases s-wave attachment was confirmed at very low energies where the DA cross-sections showed an energy dependence between $E^{-1 / 2}$ and $E^{-1}$ (Klar et al., 2001b; Schramm et al., 2002). For $\mathrm{CH}_{2} \mathrm{Br}_{2}$ (which has similar dipole moment and polarizability as $\mathrm{CH}_{3} \mathrm{I}$ ) a clear VFR just below the onset for the $v_{3}=1$ symmetric $\mathrm{CBr}_{2}$ stretch vibration was observed (Schramm et al., 2002). While clear cusp structure was detected at several vibrational thresholds for $\mathrm{CFCl}_{3}$ (Klar et al., 2001b), such structure was nearly absent for $\mathrm{CBrCl}_{3}$ (Schramm et al., 2002). $R$-matrix calculations demonstrated that $\mathrm{Br}^{-}$formation from DA to $\mathrm{CBrCl}_{3}$ 
proceeds by s-wave attachment to the ground anion state at low energies while a broad peak, observed around $0.6 \mathrm{eV}$ and evolving predominantly into the statistically favoured $\mathrm{Cl}^{-}$channel, is due to an excited anion state.

\section{A.3. Electron Scattering from cis- and trans-Difluoroethenes}

Use of polyatomic organic compounds allows introducing a "chemical dimension" into the study of electron scattering by designing molecules with the desired physical and electronic properties. Cis- and transdifluoroethenes $\left(1,2-\mathrm{C}_{2} \mathrm{H}_{2} \mathrm{~F}_{2}\right)$ represent an example where use is made of this possibility. Both molecules are virtually identical in terms of size, the nature of chemical bonding and of electronic structure, but differ dramatically in the magnitudes of the permanent dipole moments $(2.42$ Debye in cis and zero by symmetry in trans). This pair of compounds thus allowed Allan et al. (2002) to study the effect of dipole moment on the threshold peaks, while keeping the effect of other factors like polarizability, presence or absence of double bonds, number of halogens, etc., unchanged.

Figure 24 illustrates the trivial effect on the elastic cross-section. The size of the elastic cross-section is strongly enhanced at low energies by the longrange force of the permanent dipole moment of cis-difluoroethene, as expected.

Figures 25 and 26 compare the cross-sections of the $\mathrm{C}=\mathrm{C}$ stretch and the $\mathrm{C}-\mathrm{F}$ stretch vibrations for the two isomers. A band due to the $\pi^{*}$ shape resonance is seen for both types of vibrations, yielding vertical electron attachment energies of $2.37 \mathrm{eV}$ for cis-difluoroethene and $2.05 \mathrm{eV}$ for transdifluoroethene. The resonances thus lie slightly higher than in the parent compound ethene, which has a resonance at $1.78 \mathrm{eV}$ (Jordan and Burrow, 1978). The effect of the fluorine substituents may be viewed as a stabilizing inductive effect due to the large electronegativity of fluorine and a destabilizing conjugative effect due to admixture of the occupied lone pair orbitals of $\pi$ symmetry on the fluorines. The higher energy of the resonance in the cis- compound can be visualized within this picture as due to destabilizing through-space interaction of the fluorine lone pairs of $\pi$ symmetry.

Both the $\mathrm{C}=\mathrm{C}$ stretch vibrations (Fig. 25) and the $\mathrm{C}-\mathrm{F}$ stretch vibrations (Fig. 26) are excited to about the same degree in the $\pi^{*}$ shape resonance region. This reflects the fact that the $\pi^{*}$ antibonding orbital, which is temporarily occupied in the shape resonance, is antibonding with respect to both the $\mathrm{C}=\mathrm{C}$ and the $\mathrm{C}-\mathrm{F}$ distances, and is little influenced by whether the fluorines are arranged cis or trans. 


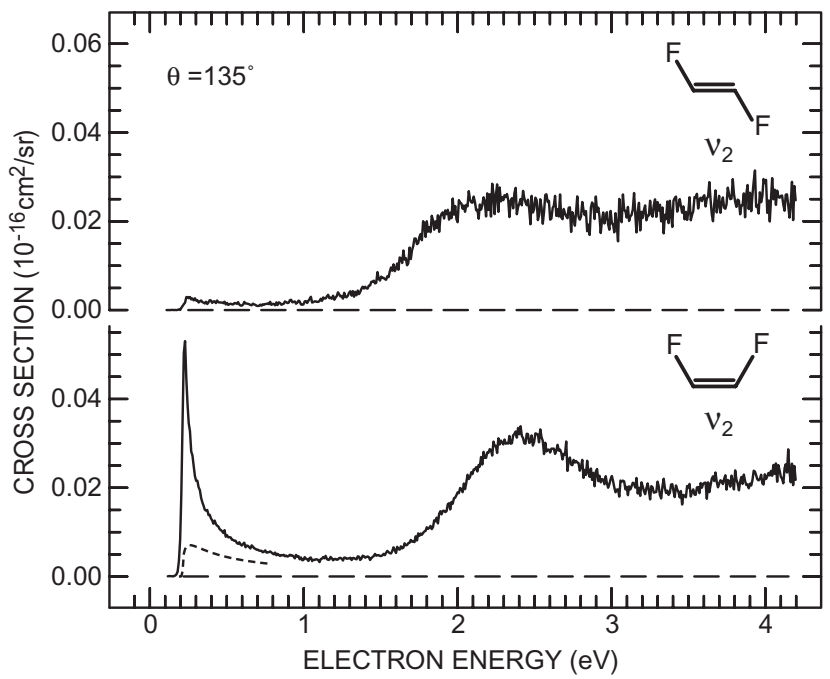

FIG. 25. Cross-sections for the excitation of the $\mathrm{C}=\mathrm{C}$ stretch vibrations $\nu_{2}$ in cis- and transdifluoroethenes. The dashed curve is a cross-section calculated from the infrared intensities using the Born approximation. $v_{2}$ is IR inactive in the trans compound and the Born crosssection is consequently zero. (from Allan et al., 2002).

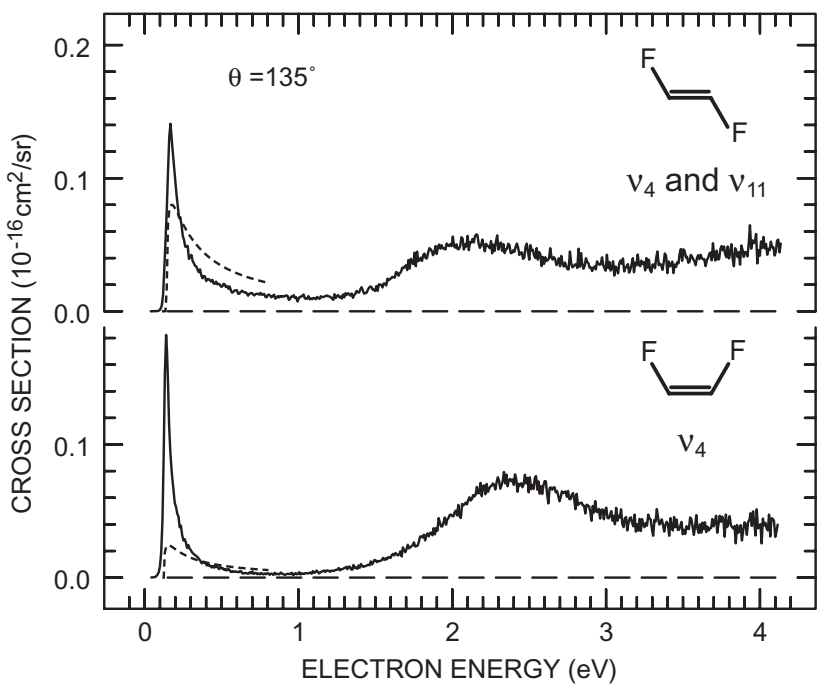

FIG. 26. Cross-sections for the excitation of the C-F stretch vibrations in cis- and transdifluoroethenes ( $v_{4}$ in cis-difluoroethene and overlaping $v_{4}$ and $v_{11}$ in trans-difluoroethene). Born cross sections are shown dashed (from Allan et al., 2002). 
Substantial differences appear in the threshold region, however. These differences were discussed in terms of two effects. The first is the trivial excitation of infrared active vibrations near threshold by the dipole mechanism. This excitation is forward-peaked and its extent in Figs. 25 and 26 has been reduced by the large scattering angle of $135^{\circ}$. The remaining contribution of the excitation by this mechanism was estimated by calculating the cross-sections within the Born approximation from the experimental infrared band intensities. This approximation is primarily intended for forward scattering, but can be assumed to be qualitatively useful even at the large scattering angle. Threshold cross-sections exceeding substantially the Born prediction were interpreted as 'true' threshold peaks, in the same sense as in the examples of HF and other halogen halides described above.

Two observations are made in the excitation of the $\mathrm{C}=\mathrm{C}$ stretch vibrations in Fig. 25. This vibration is excited at threshold only in the cis isomer where it is infrared active, and nearly absent in the trans isomer where it is infrared inactive. The cross-section in the former case is, in addition, substantially higher than the Born prediction.

Threshold peaks are found in the cross-sections for the $\mathrm{C}-\mathrm{F}$ stretch excitation for both the cis and the trans isomers in Fig. 26, but only in the cis isomer, with permanent dipole moment, does the observed cross-section substantially exceed the Born prediction. (Two $\mathrm{C}-\mathrm{F}$ stretch vibrations overlap in the trans compound and both the experimental cross-section and the Born prediction are the sums for both vibrations.). It was consequently concluded that two conditions strongly contribute to the presence of 'true' threshold peaks. One is a permanent dipole moment and the other a dipole moment being a function of nuclear coordinate for the normal mode in question (i.e., the mode must be IR active). This conclusion holds also for a number of other normal modes of cis and trans difluoroethenes measured by Allan et al. (2002) but not shown here.

These conclusions are compatible with the notion that the threshold peaks are closely related with a negative ion state where an electron is bound (in an electronic sense) by a dipole force in a spatially diffuse wave function, but only for a certain range of configurations of the nuclei. HF is a prototype of this mechanism and the present molecules extend it to a case with many normal modes and a variable permanent dipole moment. The permanent dipole moment is important by providing a sufficient 'dipole binding' in the first place. The binding energy is further enhanced and becomes a function of vibrational coordinate (for the normal mode in question) for IR active vibrations. The permanent dipole moment is not absolutely indispensable, however, as exemplified by $\mathrm{CO}_{2}$ and $\mathrm{CS}_{2}$. A sufficiently strong polarization force can replace its effect. 


\section{B. Electron Collisions with Nonpolar Molecules}

In this subsection we shall discuss molecules which do not possess a permanent electric dipole moment (i.e. $\mu=0$ ). Most of these molecules exhibit, however, a permanent electric quadrupole moment $Q \neq 0$, leading to an interaction $V_{\mathrm{Q}}(r) \propto-1 / r^{3}$ which has to be considered in addition to the polarization interaction $V_{\mathrm{pol}}(r) \propto-1 / r^{4}$. Here we consider the three cases $\mathrm{Na}_{2}, \mathrm{~F}_{2}$ and $\mathrm{Cl}_{2}$ for which we focus on the threshold behaviour of the electron attachment cross-section. Very few molecules, characterized by a high symmetry, neither possess a permanent electric dipole moment nor a permanent electric quadrupole moment, among them $\mathrm{SF}_{6}, \mathrm{CCl}_{4}$ and $\mathrm{C}_{60}$. These molecules are known as very efficient electron scavengers, and $\mathrm{SF}_{6}$ is being used as a gaseous dielectric in many applications (Christophorou and Olthoff, 2000). We further discuss the low-energy scattering behaviour of two linear, symmetric triatomic molecules which exhibit strong enhancements of elastic and inelastic scattering through a virtual state $\left(\mathrm{CO}_{2}\right)$ or a bound anion state $\left(\mathrm{CS}_{2}\right)$ and also include the weakly polar molecule $\mathrm{N}_{2} \mathrm{O}(\mu=0.16 \mathrm{D})$ which is isoelectronic with $\mathrm{CO}_{2}$ and has exhibited intriguing vibrational structure in a recent high resolution DA study. These findings are also relevant to the observations of vibrational resonance structure made for clusters composed of these and related molecules.

\section{B.1. Electron Attachment to Vibrationally Excited Sodium Molecules}

Among the nonpolar diatomic molecules $Y_{2}$ only a few cases (including the halogen molecules) exhibit anion formation by dissociative electron attachment at near-zero electron energies. The threshold behaviour of DA to the halogen molecules is still under debate (see next subsection). DA to the alkali dimers is endothermic by a few tenths of an $\mathrm{eV}$. Zero energy attachment, may, however, be investigated if a sufficient amount of vibrational energy is present in the molecule. Here we describe the results of a recent experiment in which high resolution near-zero energy electron attachment to sodium dimers in a controlled vibrational level $v$ has been carried out (Keil et al., 1999):

$$
\mathrm{e}^{-}(E)+\mathrm{Na}_{2}\left(\mathrm{X}^{1} \Sigma_{g}^{+}, v\right) \rightarrow \mathrm{Na}_{2}^{-}\left(\mathrm{A}^{2} \Sigma_{g}^{+}\right) \rightarrow \mathrm{Na}^{-}\left({ }^{1} S\right)+\mathrm{Na}\left(3^{2} S\right) .
$$

Figure 27 illustrates the relevant potential energy curves (Külz et al., 1996, Keil et al., 1999), as obtained in a high level electronic structure calculation. The electronic configuration of the $\mathrm{Na}_{2}^{-}\left({ }^{2} \Sigma_{\mathrm{u}}{ }^{+}\right)$ground state (chain curve) is $1 \sigma_{\mathrm{g}}^{2} 1 \sigma_{\mathrm{u}}$; it is stable for lower vibrational levels (adiabatic electron 


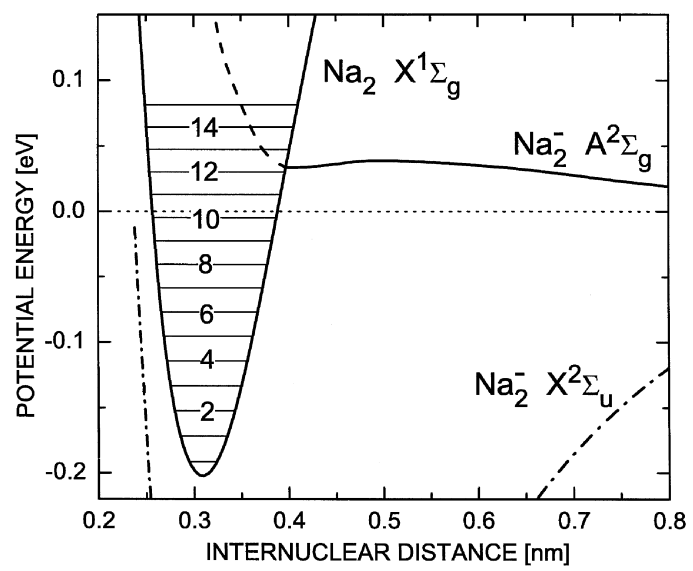

FIG. 27. Potential energy curves for $\mathrm{Na}_{2}$ and $\mathrm{Na}_{2}^{-}$, relevant for threshold electron attachment to $\mathrm{Na}_{2}(v>0)$ molecules (from Keil et al., 1999).

affinity $+0.44 \mathrm{eV}$ ) and is not involved in the processes discussed here. The $\mathrm{Na}_{2}^{-}\left(\mathrm{A}^{2} \Sigma_{\mathrm{g}}{ }^{+}\right)$state is stable for internuclear distances $R>R_{\mathrm{C}}=0.38 \mathrm{~nm}$ and turns into a resonance state for smaller $R$. At long range it is dominated by the $1 \sigma_{\mathrm{g}} 1 \sigma_{\mathrm{u}}^{2}$ configuration. It acquires significant admixture of a $1 \sigma_{\mathrm{g}}^{2} 2 \sigma_{\mathrm{g}}$ configuration at distances below $0.5 \mathrm{~nm}$. For $R<R_{\mathrm{C}}$ the $2 \sigma_{\mathrm{g}}$ orbital turns into a continuum wave function; anion formation involves a two-electron process in which the incoming s-wave electron and one of the molecular $1 \sigma_{\mathrm{g}}$ electrons form the $1 \sigma_{\mathrm{u}}^{2}$ part of the Feshbach-type $\mathrm{Na}_{2}^{-}\left(\mathrm{A}^{2} \Sigma_{\mathrm{g}}{ }^{+}\right)$resonance state. Reaction (37) becomes exothermic for $v=11$, but due to the barrier in the $\mathrm{Na}_{2}^{-}\left(\mathrm{A}^{2} \Sigma_{\mathrm{g}}^{+}\right)$potential energy curve near $0.47 \mathrm{~nm}$ the DA crosssections for both $v=11$ and 12 are predicted to be suppressed at very low energies.

Using a collimated supersonic beam, containing both $\mathrm{Na}(3 \mathrm{~s})$ atoms and $\mathrm{Na}_{2}(v=0)$ molecules, the experiment (Keil et al., 1999) combined the laser photoelectron attachment method (here two-step photoionization of $\mathrm{Na}(3 \mathrm{~s})$ through the excited $\mathrm{Na}\left(3 \mathrm{p}_{3 / 2}\right)$ level) with selective vibrational excitation by means of a two-photon Raman technique with acronym STIRAP (Stimulated Raman with Adiabatic Passage) (Vitanov et al., 2001). $\mathrm{Na}^{-}$ ions due to the DA process (37) are detected with a time-of-flight mass spectrometer. In Fig. 28 the attachment spectra for the four selected vibrational levels $v=12,13,14$, and 22 are shown; they were obtained at an electron current of about $0.2 \mathrm{nA}$. There is a clear change in the threshold behaviour when going from $v=12$ to higher vibrational levels; for $v \geq 13$, the calculations and the experimental results both exhibit a strong rise of the 


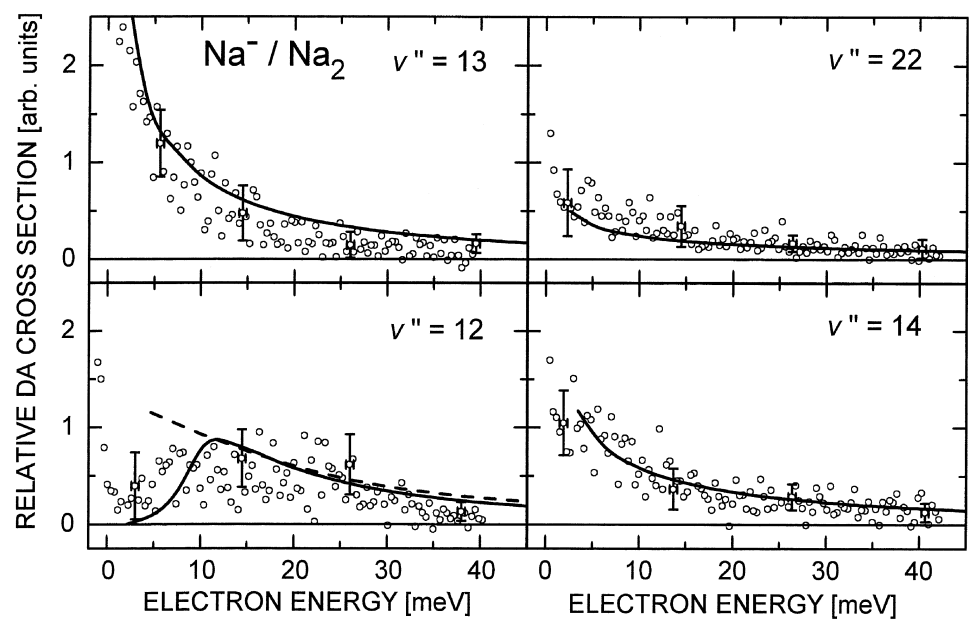

FIG. 28. Electron attachment cross-sections for selectively vibrationally excited $\mathrm{Na}_{2}(v)$ molecules (from Keil et al., 1999).

cross-section towards zero electron energy which is characteristic for s-wave capture. Note that the theoretical data for $v=12$ (which show a sharp rise above $8 \mathrm{meV}$ ) have been convoluted by a Gaussian with $6 \mathrm{meV}$ width to simulate the effects of experimental resolution. The overall agreement between the experimental and theoretical results is quite satisfactory although the experimental anion yields seem to decrease somewhat more rapidly towards higher energies than the theoretical cross-sections.

\section{B.2. Electron Attachment to Fluorine and Chlorine Molecules}

Dissociative electron attachment to the halogen molecules $Y_{2}(Y=\mathrm{F}, \mathrm{Cl}$, $\mathrm{Br}, \mathrm{I})$ at energies below $1 \mathrm{eV}$ is expected to occur through formation of the lowest negative-ion state with ${ }^{2} \Sigma_{\mathrm{u}}{ }^{+}$symmetry according to the scheme (see, e.g., Domcke, 1991; Christophorou and Olthoff, 1999)

$$
\mathrm{e}^{-}(E)+Y_{2}\left(\mathrm{X}^{1} \Sigma_{\mathrm{g}}^{+}\right) \rightarrow Y_{2}^{-}\left({ }^{2} \Sigma_{\mathrm{u}}^{+}\right) \rightarrow Y^{-}\left({ }^{1} S\right)+Y\left({ }^{2} P\right) .
$$

This process has been observed in several experimental studies (e.g. Kurepa and Belić, 1978, Tam and Wong, 1978, Chantry, 1982) as a peak at or close to zero energy with a width which was to a large extent limited by the experimental resolution (between 0.08 and $0.2 \mathrm{eV}$ ). For $F_{2}$ Chutjian and Alajajian (1987) reported s-wave behaviour of the DA cross-section at an energy width of $6-12 \mathrm{meV}$, using the TPSA method. From a theoretical 
point of view the process is expected to be dominated by the p-wave because of the ungerade parity of the resonance state which rules out s-wave attachment. According to the Wigner threshold law the cross-section should thus grow with the square root of the electron energy at sufficiently low energies, as predicted in several theoretical calculations for $\mathrm{F}_{2}$ (Hazi et al., 1981; Bardsley and Wadehra, 1983; Brems et al., 2002) and for $\mathrm{Cl}_{2}$ (Fabrikant et al., 2000).

Brems et al. (2002) carried out high level ab initio calculations of the lowest $\mathrm{F}_{2}^{-}$resonance state by the $R$-matrix method with the Feshbach-Fano partitioning technique to treat the nuclear dynamics. Their DA cross-section exhibits p-wave threshold behavior and reaches a peak around $0.2 \mathrm{eV}$ with a size of about $1.5 \times 10^{-20} \mathrm{~m}^{2}$. The experimental DA cross-section of Chantry (1982) is in satisfactory agreement with the theoretical results over the range $0.6-2 \mathrm{eV}$, but the increase towards lower energies (compatible with the TPSA data) remains unexplained. There is clearly a need for a new highresolution experiment with the aim to clarify the situation.

For the chlorine molecule, a similar discrepancy as for $\mathrm{F}_{2}$ appeared to exist. A critical analysis of the early and more recent low resolution data (Kurepa and Belic, 1978; Tam and Wong, 1978; Feketeova et al., 2003), based on their comparison with convoluted theoretical cross-section shapes (using appropriate energy distribution functions), reveals that they are in fact not contradictory to p-wave attachment (Ruf et al., 2003). In a recent LPA experiment (energy width near threshold $1 \mathrm{meV}$ ) Barsotti et al. (2002b) provided conclusive experimental evidence for $\mathrm{p}$-wave behaviour of the attachment cross-section for $\mathrm{Cl}_{2}$ by demonstrating the steep rise from threshold to a maximum located around $50 \mathrm{meV}$.

Recent accurate calculations (Leininger and Gadea, 2000) of potential curves for $\mathrm{Cl}_{2}$ and $\mathrm{Cl}_{2}^{-}$provide useful information regarding the dynamics of the process. The curve crossing between the $\mathrm{Cl}_{2}\left(\mathrm{X}^{1} \Sigma_{\mathrm{g}}^{+}\right)$and $\mathrm{Cl}_{2}^{-}\left({ }^{2} \Sigma_{\mathrm{u}}^{+}\right)$states occurs below the left classical turning point for the nuclear motion in $\mathrm{Cl}_{2}$. Typically the Franck-Condon factor changes relatively slowly in the nearthreshold region, and the energy dependence of the cross-section is mainly determined by the capture width $\Gamma$ which gives the $E^{-1 / 2}$ law in the case of s-wave attachment and the $E^{1 / 2}$ law in the case of the p-wave attachment. However, in the case of attachment to $\mathrm{Cl}_{2}$ the Franck-Condon factor drops very rapidly with rising energy, therefore the DA cross-section should exhibit a rather narrow peak near threshold with the $E^{1 / 2}$ behavior limited to energies below $10 \mathrm{meV}$.

To confirm this conjecture, the DA cross-section was calculated by Fabrikant et al. (2000), using the resonance $R$-matrix theory. The surface amplitude $\gamma$ was adjusted to reproduce the recommended value of the DA cross-section (Christophorou and Olthoff, 1999) at $0.1 \mathrm{eV}$ and the 


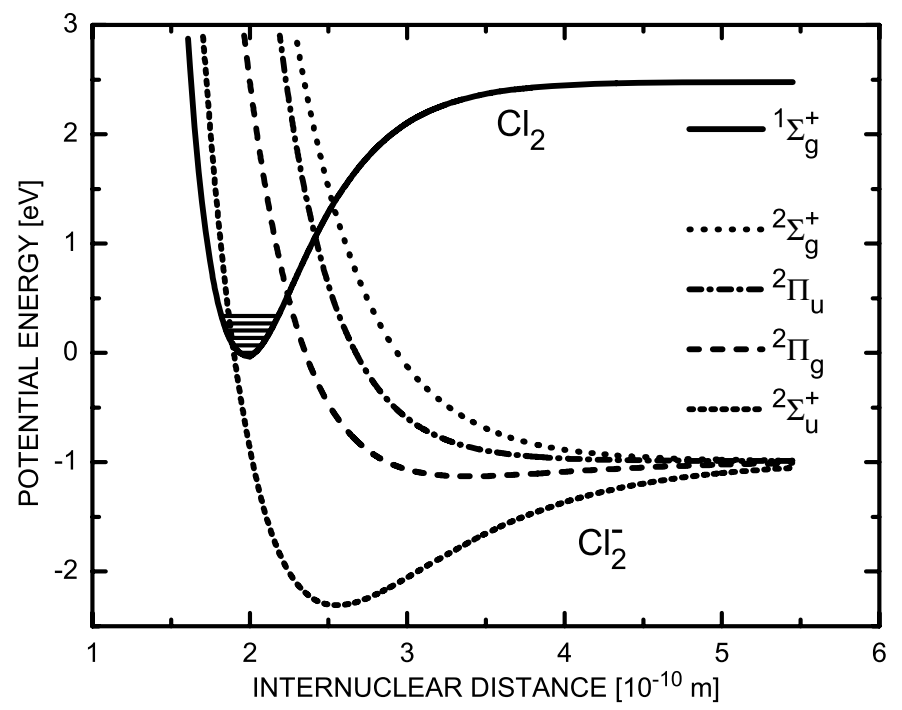

FIG. 29. Potential energy curves for $\mathrm{Cl}_{2}$ and $\mathrm{Cl}_{2}^{-}$, relevant for electron attachment to $\mathrm{Cl}_{2}\left(\mathrm{X}^{1} \Sigma_{\mathrm{g}}^{+}\right)$molecules (from Barsotti et al., 2002b).

magnitude of the swarm-derived DA rate coefficients (McCorkle et al., 1984). In Fig. 29 we present the fitted potential curves of the problem. The neutral curve $V(\rho)$ and the "diabatic" curve $U(\rho)$ were parameterized by Morse functions.

In Fig. 30 we show the calculated DA cross-section (full curve) in the low-energy region $(0-200 \mathrm{meV})$ and compare it with the measured LPA cross-section (open circles, normalized in absolute size to the calculated value at the maximum, assuming a vibrational temperature of $\mathrm{Cl}_{2}$ of $T_{\mathrm{i}}=500 \mathrm{~K}$ ). The shapes of the experimental and theoretical cross-sections are in good agreement. We emphasize that the shape of the DA cross-section for different initial vibrational levels $v_{\mathrm{i}}$ is almost independent of $v_{\mathrm{i}}$ (see broken line in Fig. 30, calculated with $T_{\mathrm{i}}=300 \mathrm{~K}$ ) while the absolute size rises strongly with increasing $v_{\mathrm{i}}$, reflecting mainly the changes of the Franck-Condon factors. The rather sharp decrease of the DA crosssection above $E=0.05 \mathrm{eV}$ is caused by two reasons: the fast drop of the Franck-Condon factor with rising energy mentioned above and the decrease of the survival probability of the intermediate negative ion against autodetachment. It is essential that this behavior is independent of our normalization procedure. If we vary $\gamma$, but keep the adiabatic anion curve fixed, the shape of the cross-section does not change.

The $R$-matrix calculations were extended to provide semiempirical predictions for vibrational excitation (VE) cross-sections for this molecule of 


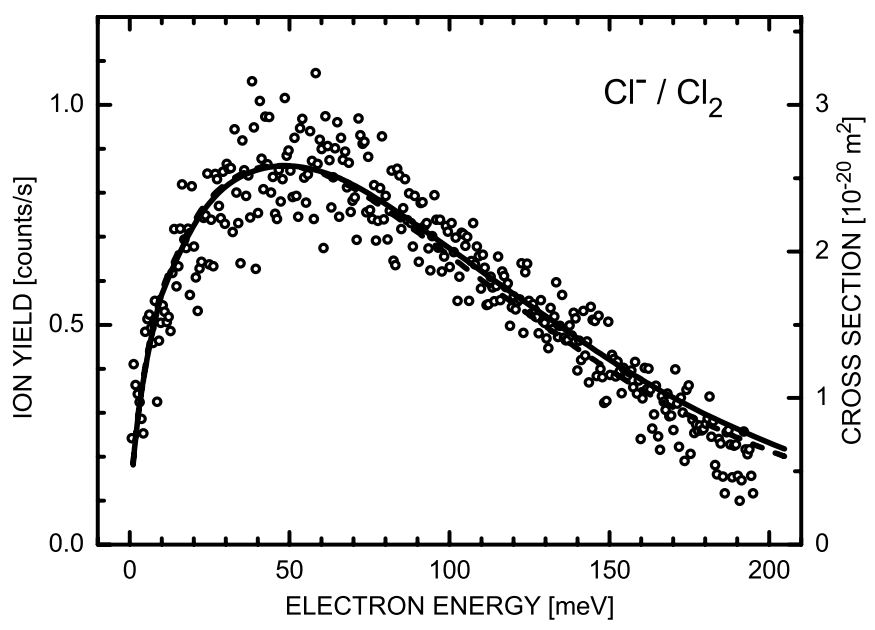

FIG. 30. Cross-section for dissociative electron attachment to $\mathrm{Cl}_{2}$. The absolute scale refers to the calculation which assumes a vibrational temperature of $T_{\mathrm{i}}=500 \mathrm{~K}$ (full curve). The LPA data (open circles) and the calculation with $T_{\mathrm{i}}=300 \mathrm{~K}$ (broken curve) have been normalized to the maximum of the full curve (from Barsotti et al., 2002b).

substantial practical interest, not only though the $\mathrm{Cl}_{2}^{-}\left({ }^{2} \Sigma_{\mathrm{u}}{ }^{+}\right)$resonance, but also through the higher lying ${ }^{2} \Pi_{\mathrm{g}}$ and ${ }^{2} \Pi_{\mathrm{u}}$ resonances (Ruf et al., 2003). So far no direct measurements for these VE cross-sections have been carried out.

\section{B.3. Electron Attachment to $\mathrm{SF}_{6}$ and $\mathrm{CCl}_{4}$}

Both $\mathrm{SF}_{6}$ and $\mathrm{CCl}_{4}$ belong to the few molecules for which the Vogt-Wannier capture model should be applicable in view of missing electric dipole and quadrupole moments, and it is thus of interest to compare the prediction of the VW theory with experimental results for the energy dependent attachment cross-section, obtained at very low energies and high resolution. To illustrate the importance of the reactive attachment channel, we also present the total electron scattering cross-sections.

Using energy-variable photoelectrons from VUV photoionization of rare gas atoms (energy range $0-160 \mathrm{meV}$, energy width $6-8 \mathrm{meV}$ ), Chutjian and Alajajian (1985) obtained clear evidence for s-wave behavior of the attachment cross-section for $\mathrm{SF}_{6}$ and $\mathrm{CCl}_{4}$ at low energies. They used the following analytical form for the attachment cross-section to describe the TPSA data over the range from 0 to $140 \mathrm{meV}$ (see also Chutjian, 1992; Chutjian et al., 1996):

$$
\sigma_{\mathrm{TPSA}}(E)=N_{\mathrm{TPSA}}\left[a E^{-1 / 2} \exp \left(-E^{2} / \lambda^{2}\right)+\exp (-E / \gamma)\right] .
$$


It contains three fit parameters $a, \lambda$, and $\gamma$ and a normalization constant $N_{\text {TPSA }}$; the latter is determined by use of Eq. (35). The analytical form of the cross-section (39) has the deficiency that the second exponential term (which serves to describe the fast decrease of the cross-section at higher energies) is not cut off towards very low energies where the first term (which describes the limiting s-wave behavior) should take over. As long as the first term is not very much larger than the second one, this leads to a more or less substantial perturbation of the s-wave term (Klar et al., 2001a).

Subsequently, Klar et al. (1992a,b, 2001a), Hotop et al. (1995) and Schramm et al. (1998) used the laser photoelectron attachment (LPA) method to investigate anion formation from these two molecules over a similar energy range, but with substantially reduced energy width (below $1 \mathrm{meV}$ ). As an important ingredient and improvement over previous work, they analyzed the effects of residual electric fields (reduced to values below $1 \mathrm{~V} / \mathrm{m}$ ) on the near-threshold attachment yield through model calculations of the attachment yield (Klar et al., 1992b; 1994a, b; 2001a, b; Schramm et al., 1998). In the spirit of the s-wave capture formula (21) due to Klots they used the analytical cross-section

$$
\sigma_{\mathrm{e}}(E)=\left(\sigma_{0} / E\right)\left[1-\exp \left(-\beta E^{1 / 2}\right)\right]
$$

which was found to provide a very good description of the experimental attachment yield from threshold up to the first vibrationally inelastic onset for both $\mathrm{SF}_{6}$ (Klar et al., 1992b; 1994a, b) and $\mathrm{CCl}_{4}$ (Hotop et al., 1995; Klar et al 2001a). In this way they were able to determine the parameter $\beta$ in Eq. (40) to within $10 \%$ and thereby quantify the deviations of the cross section from the limiting behavior $\sigma_{\mathrm{e}}(E \rightarrow 0)$ which - in terms of Eq. (40) - is given by $\sigma_{\mathrm{e}}(E \rightarrow 0)=\sigma_{0} \beta / E^{1 / 2}$. With $\beta$ expressed in units of $(\mathrm{meV})^{-1 / 2}$, Klar et al. obtained $\beta=0.405(40)$ for $\mathrm{SF}_{6}$ (Klar et al., 1992b) and $\beta=0.59$ (6) for $\mathrm{CCl}_{4}$ (Hotop et al., 1995; Klar et al., 2001a), in both cases distinctly larger than the prediction obtained from the Klots formula (21), namely $\beta_{\mathrm{K}}=0.228$ for $\mathrm{SF}_{6}$ and $\beta_{\mathrm{K}}=0.299$ for $\mathrm{CCl}_{4}$. For $\mathrm{SF}_{6}, \mathrm{Schramm}$ et al. (1998) measured the attachment yield at residual electric fields as low as $0.01 \mathrm{~V} / \mathrm{m}$ and negligible laser bandwidth for electron energies from $10 \mathrm{meV}$ down to $20 \mu \mathrm{eV}$; they confirmed the results of Klar et al. (1992b) for the parameter $\beta$. The limiting LPA rate coefficient $k_{\mathrm{e}}(E \rightarrow 0)=$ $\sigma_{\mathrm{e}}(E \rightarrow 0)\left(2 E / m_{\mathrm{e}}\right)^{1 / 2}=\sigma_{0} \beta\left(2 / m_{\mathrm{e}}\right)^{1 / 2}\left(5.4(8) \times 10^{-7} \mathrm{~cm}^{3} \mathrm{~s}^{-1}\right)$ is in good agreement with the Vogt-Wannier prediction $k_{\mathrm{c}}=5.15 \times 10^{-7} \mathrm{~cm}^{3} \mathrm{~s}^{-1}$ (Klar et al., 1992b) and with RET data for $\mathrm{SF}_{6}^{-}$formation from $\mathrm{SF}_{6}$ at high principal quantum numbers $n\left(k_{n l}=4.0(10) \times 10^{-7} \mathrm{~cm}^{3} \mathrm{~s}^{-1}\right.$, Ling et al., 1992; Klar et al., 1994b; Dunning, 1995). 


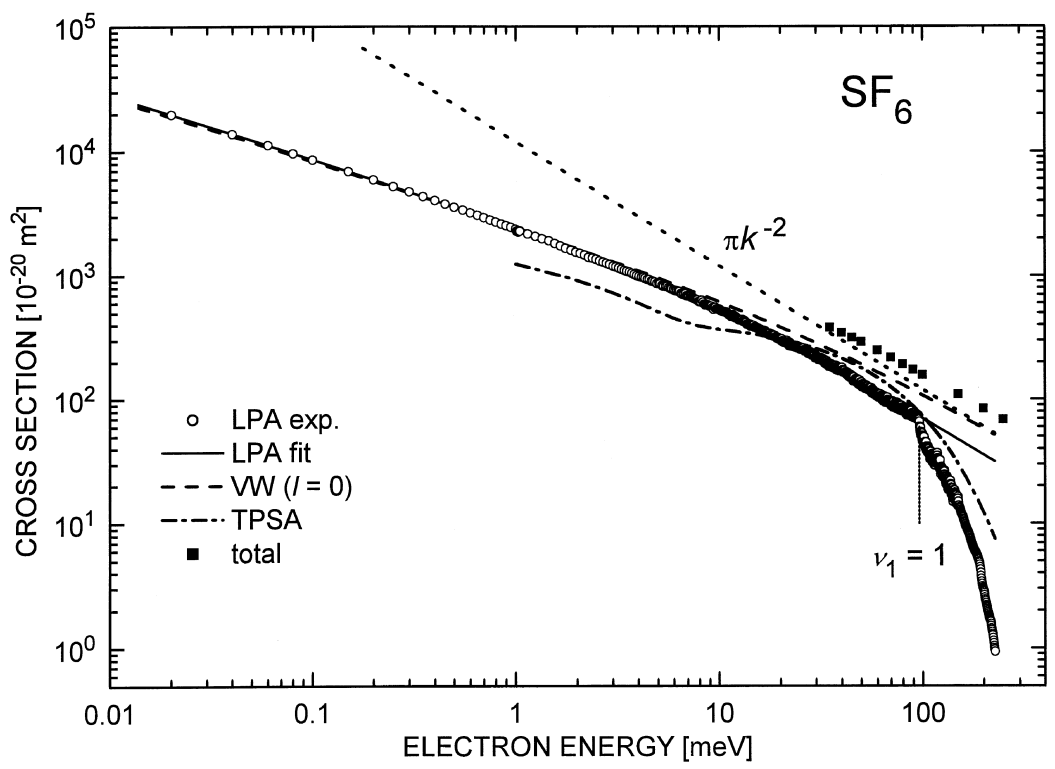

FIG. 31. Absolute cross-sections for electron collisions with $\mathrm{SF}_{6}$. LPA: Laser photoelectron attachment $\left(\mathrm{SF}_{6}^{-}\right.$formation, Klar et al., 1992b; Hotop et al., 1995), TPSA: Threshold photoelectron attachment ( $\left(\mathrm{SF}_{6}^{-}\right.$formation, Chutjian and Alajajian, 1985), see text. For comparison, we have included the total cross-sections due to Ferch et al. (1982) (full squares), the Vogt-Wannier s-wave capture cross-section $\mathrm{VW}(l=0)$, the limiting s-wave reaction cross section $\pi k^{-2}$ and the LPA fit according to Eq. (40).

In Fig. 31 we present a comparison of the LPA cross-section (normalized in absolute size to the thermal rate coefficient due to Petrovic and Crompton, 1985) with the VW result for s-wave attachment (p-wave capture would reach its peak at an electron energy of about $330 \mathrm{meV}$ and is neglected), with the fit (39) to the TPSA data (Chutjian and Alajajian, 1985) and with the empirical fit (40) of Klar et al. (1992b). The fit (40) gives a good description of the LPA data up to the threshold for $v_{1}=1$ vibrational excitation where the cross-section exhibits a sharp downward cusp, predicted theoretically by Gauyacq and Herzenberg (1984) and confirmed by Klar et al. (1992a, b). Systematic deviations between the LPA and TPSA results are observed (in spite of using the same thermal rate coefficient for normalization) here and also for other molecules, as discussed and explained by Klar et al. (1992b; 1994a; 2001a, b) and Schramm et al. (1999, 2002). In a more recent study Howe et al. (2001) confirmed the smooth Klots-type behavior of the $\mathrm{SF}_{6}^{-}$cross-section (see also below).

For comparison with the (nondissociative) attachment channel, we have included in Fig. 31 the total scattering cross-section, as presented in the 
survey of Christophorou and Olthoff (2000, Table 9) and due to Ferch et al. (1982) at low energies. Below the onset for vibrational excitation of the symmetric stretch mode $v_{1}=1$, the total cross-section is $2.23(90 \mathrm{meV})$ to 2.05 $(35 \mathrm{meV})$ times higher than the fitted LPA attachment cross-section. A decrease of the ratio between the total cross-section and the attachment cross-section towards very low energies is expected on theoretical grounds; the ratio should be a linear function of wave vector $k$ at very low energies and take the value unity for $E \rightarrow 0$. Assuming that VE cross-sections of the lower energy modes (e.g. $v_{6}=1$, onset at $44 \mathrm{meV}$ ) can be neglected, the elastic scattering cross-section $\sigma_{\mathrm{ES}}$ can be calculated from the difference between the total cross-section and the LPA attachment cross-section. The resulting values for $\sigma_{\mathrm{ES}}$ rise from $95 \times 10^{-20} \mathrm{~m}^{2}$ at $90 \mathrm{meV}$ to $195 \times 10^{-20} \mathrm{~m}^{2}$ at $35 \mathrm{meV}$ while the LPA cross-section rises from $78 \times 10^{-20} \mathrm{~m}^{2}$ to $185 \times 10^{-20} \mathrm{~m}^{2}$, respectively. A preliminary analysis of these data (Fabrikant, unpublished) within the framework of the modified effective range theory (O'Malley et al., 1962) yields two possible solutions for the electron scattering length of $\mathrm{SF}_{6}$, namely $A \approx 7.3 \mathrm{a}_{0}$ and $A \approx-10.0 \mathrm{a}_{0}$. In the first case a weakly bound state would exist for which no evidence exists so far (Christophorou and Olthoff, 2000). The second case would indicate a virtual state and would make the case of low-energy e- $\mathrm{SF}_{6}$ scattering similar to that of $\mathrm{CO}_{2}$ (see below). In order to clarify the situation, new measurements of the total cross-sections down to very low energies are needed. In recent theoretical calculations of electron scattering from $\mathrm{SF}_{6}$ over the broad energy range from a few $\mathrm{meV}$ to $100 \mathrm{eV}$, Gianturco and Lucchese (2001) obtained total cross-sections which are substantially lower than the experimental results at energies below $1 \mathrm{eV}$. They attributed the differences to inelastic channels (vibrational excitation), but did not consider electron attachment which is the major reaction channel at the lowest energies. Their elastic cross-section at $35 \mathrm{meV}$ is about 40 times lower than the estimate given above.

Since in the case of s-wave scattering there is no centrifugal barrier to support the resonance state, the process of low-energy attachment can be viewed as a direct nonadiabatic capture (Crawford and Koch, 1974; Gauyacq, 1982). Attachment to $\mathrm{SF}_{6}$ was discussed in terms of nonadiabatic coupling by Gauyacq and Herzenberg (1984): the low-energy electron can give up its energy to become bound if the crossing of the negative-ion curve with the neutral curve occurs close to the equilibrium internuclear separation. However, there should be a mechanism that is preventing the electron from escaping into the continuum. In the case of $\mathrm{SF}_{6}$ this occurs due to a fast intramolecular vibrational redistribution (IVR) of the available energy over many vibrational modes, before the nuclear framework can oscillate back to its initial configuration (Gauyacq and Herzenberg, 1984). 
The $\mathrm{SF}_{6}^{-}$anion becomes metastable, and this explains the nondissociative feature of low-energy attachment to $\mathrm{SF}_{6}$ (see also Thoss and Domcke, 1998). Since the capture in this case is nonresonant, the VW model becomes appropriate. Of course a $-\alpha /\left(2 r^{4}\right)$ singularity, which plays an essential role in the VW model, is unphysical. However, for sufficiently high $\alpha$ it describes quite well the probability to find the electron within the molecule where the direct energy exchange is likely to occur.

The metastability of the $\mathrm{SF}_{6}^{-}$anion has been the subject of many studies aiming to determine its lifetime (see Christophorou and Olthoff, 2000, Suess et al., 2002, and references therein). This lifetime is expected to depend on the internal energy (i.e. vibrational energy) of the anion and on the energy $E$ of the electron attached in the primary capture process. Using a permanent magnet Penning trap, Suess et al. (2002) measured the lifetime of $\mathrm{SF}_{6}^{-}$ions, produced by electron transfer from $\mathrm{K}^{* *}(30 \mathrm{p})$ and $\mathrm{K}^{* *}(60 \mathrm{p})$ Rydberg atoms to a beam of thermal $(300 \mathrm{~K}) \mathrm{SF}_{6}$ molecules. In both cases, they obtained a lifetime of about $10 \mathrm{~ms}$. Previously reported lifetimes for $\mathrm{SF}_{6}^{-}$ions formed by free thermal electron capture depend strongly on the experimental technique used. Experiments using time-of-flight methods typically yield values of a few tens of microseconds, whereas ioncyclotron-resonance methods suggest lifetimes of a millisecond or longer (Christophorou and Olthoff, 2000). In part, these differences might be explained by differences in the $\mathrm{SF}_{6}$ gas temperature and the energy of the attached electrons, leading to $\mathrm{SF}_{6}^{-}$ions in a variety of states that autodetach at different rates. A recent time-of-flight measurement, using a jetcooled $\mathrm{SF}_{6}$ target and a laser photoelectron source (LeGarrec et al., 2001), yielded a lifetime of about $19 \mu$ s that was independent of electron energy in the range $0.4-120 \mathrm{meV}$. This value is difficult to reconcile with the much longer lifetimes of Dunning's group (Suess et al., 2002) and with the results of our laser photoelectron attachment experiments in which the $\mathrm{SF}_{6}^{-}$ions, formed by electron capture in the energy range $0-200 \mathrm{meV}$, are detected $100-200 \mu$ s after their generation at intensities which indicate that losses due to autodetachment during this time interval must be small at $T=300 \mathrm{~K}$.

At elevated temperatures, autodetachment and especially dissociation of the primary $\mathrm{SF}_{6}^{-}$anion into the products $\mathrm{SF}_{5}^{-}+\mathrm{F}$ are known to occur (Chen and Chantry, 1979). High-resolution data on the temperature dependence of the cross-sections for $\mathrm{SF}_{6}^{-}$and $\mathrm{SF}_{5}^{-}$formation have recently become available (Barsotti et al., 2003a). With the anion detected about $100 \mu$ s after their production, it was found that the general shape for the $\mathrm{SF}_{6}^{-}$crosssection varied little with a tendency that the decrease towards higher energies became somewhat steeper with rising temperature. This effect can be attributed to both autodetachment and dissociation towards $\mathrm{SF}_{5}^{-}+\mathrm{F}$. For sub-thermal temperatures (due to cooling in a supersonic expansion at 
higher stagnation pressures) cusp structure at vibrational onsets was found to become clearer. The latter finding is in agreement with recent photoelectron attachment experiments of Chutjian's group (Howe et al., 2001) in which a pulsed, seeded supersonic beam of $10 \% \mathrm{SF}_{6}$ in Xenon was used; it is expected that a considerable amount of vibrational cooling occurs in such an expansion. Howe et al. (2001) found clear cusp structure around the vibrational onsets $v_{6}, v_{1}$, and $v_{3}$ and a decrease of the cross-section which was characterized by a parameter $\beta$ (see Eq. (40)) smaller than the one obtained by Klar et al. (1992b) at $T_{\mathrm{G}}=300 \mathrm{~K}$.

For the $\mathrm{CCl}_{4}$ molecule, attachment of electrons with very low energies leads to a $\mathrm{CCl}_{4}^{-*}$ complex with a lifetime of 7.5(25) ps (Popple et al., 1996) which dissociates to the observed anion products $\mathrm{Cl}^{-}$and $\mathrm{CCl}_{3}$; only a small fraction of the available excess energy (about $0.6 \mathrm{eV}$ ) appears as translational energy (Popple et al., 1996). In the LPA study of DA to $\mathrm{CCl}_{4}$ (Hotop et al., 1995; Klar et al., 2001a; normalization of LPA cross-section to thermal rate coefficient of Orient et al., 1989) the experimental energy resolution was about $0.8 \mathrm{meV}$, as limited by residual electric fields of about $0.5 \mathrm{~V} / \mathrm{m}$ (Klar et al., 2001a). Correspondingly, the extrapolation to the VW limit is somewhat less certain than for $\mathrm{SF}_{6}$, but model calculations including the residual field and the cross-section (40) yielded very good agreement between the modelled and the measured attachment yield for $\mathrm{CCl}_{4}$ in the threshold region (Klar et al., 2001a).

In Fig. 32 we compare the recommended experimental DA cross-section for $\mathrm{CCl}_{4}$ (consisting of the LPA cross-section at energies below $173 \mathrm{meV}$ and electron beam data due to Chu and Burrow (1990) at higher energies, see also Klar et al., 2001a) with the VW s-wave prediction and the LPA fit of Klar et al. (2001a). The slope of the experimental curve is steeper than that given by the VW model. This might be indicative - according to the theoretical discussion of the DA cross-section for methyl iodide (Fig. 12) of a weakly bound negative-ion state. Indeed, as was suggested by Burrow et al. (1982) and Gallup et al. (2003), the ground state ${ }^{2} \mathrm{~A}_{1}$ of the $\mathrm{CCl}_{4}^{-}$anion is bound with a very small binding energy, whereas the first repulsive excited state ${ }^{2} \mathrm{~T}_{2}$ (to which the DA peak at $0.8 \mathrm{eV}$ is attributed) has a vertical attachment energy of $0.94 \mathrm{eV}$. It is likely that the ${ }^{2} \mathrm{~T}_{2}$ state drives the resonant DA process whereas the ${ }^{2} \mathrm{~A}_{1}$ state enhances this process at low energies.

For comparison with the reactive attachment channel, we have included in Fig. 32 the total scattering cross-section, measured at high resolution by Ziesel et al. (2003) over the range $15-200 \mathrm{meV}$. Below the onset for vibrational excitation of the symmetric stretch mode $v_{1}=1$ (where the DA cross-section exhibits a clear downward cusp) the total cross-section is nearly parallel to the DA cross-section (which is close to the unitary limit $\pi / k^{2}$ ) at 


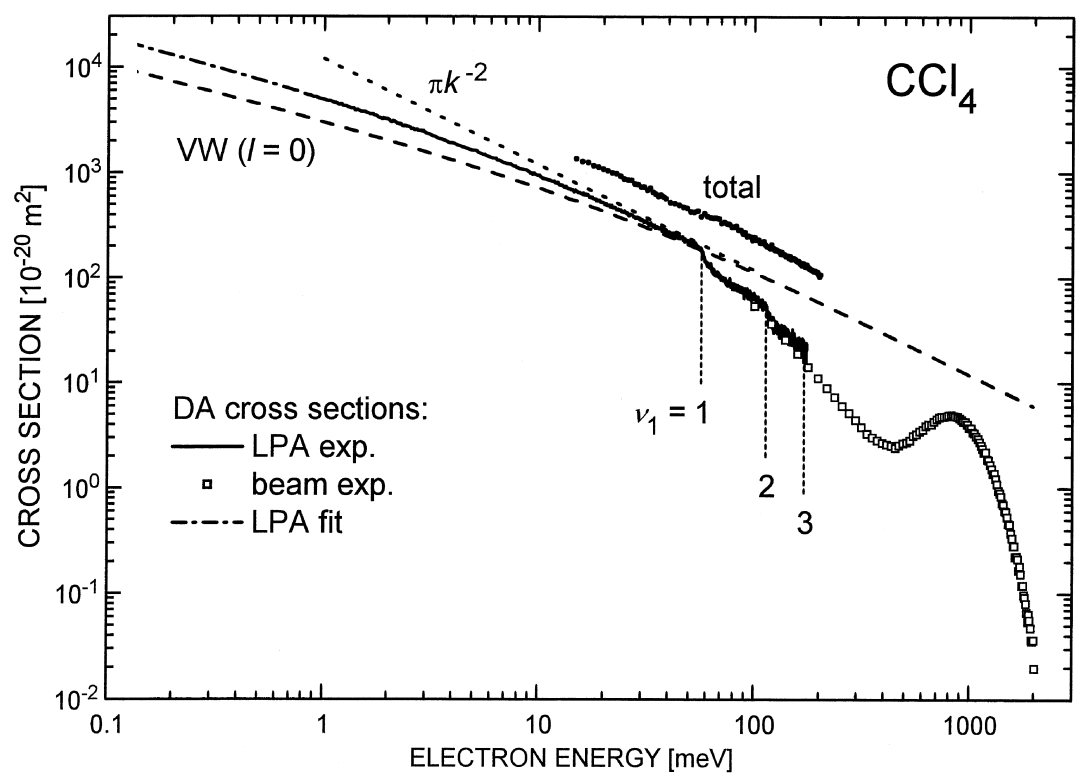

FIG. 32. Absolute cross-section for dissociative electron attachment to $\mathrm{CCl}_{4}$ molecules $\left(\mathrm{Cl}^{-}\right.$ formation, from Klar et al., 2001a), as compared to the Vogt-Wannier s-wave capture crosssection $\mathrm{VW}(l=0)$, the limiting s-wave reaction cross section $\pi / k^{2}$ and the LPA fit according to Eq. (40). For comparison the total scattering cross section (full circles, Ziesel et al., 2003) is included.

essentially twice the size. Apparently the very low energy region where the ratio between the total cross-section and the attachment cross-section becomes a linear function of wave vector $k$ has not been reached yet. Using the DA cross-section of Klar et al. (2001a) in conjunction with their total and backward scattering cross-sections (the latter measured down to $8 \mathrm{meV}$ ), Ziesel et al. (2003) derived phase shifts for s-wave and p-wave scattering over the energy range $8-55 \mathrm{meV}$. Below $25 \mathrm{meV}$, the s-wave phase shift varies rather little and takes values around $0.65 \mathrm{rad}$. In order to reach the expected limit of 0 or $\pi \mathrm{rad}$ (in the absence or presence of a bound state compatible with s-wave symmetry) the s-wave phase shift has to change dramatically from $8 \mathrm{meV}$ down to $0 \mathrm{meV}$; this is in sharp contrast to the weak variation in the range $8-25 \mathrm{meV}$.

In Fig. 33a we present the energy-dependent rate coefficient $k_{\mathrm{e}}(E)$ for free electron attachment to $\mathrm{CCl}_{4}$ over the range $(0.8-173) \mathrm{meV}$ (Hotop et al., 1995; Klar et al., 2001a). The limiting LPA value $12.3(19) \times 10^{-7} \mathrm{~cm}^{3} \mathrm{~s}^{-1}$ (full circle, Klar et al., 2001a) is in good agreement with the most recent 

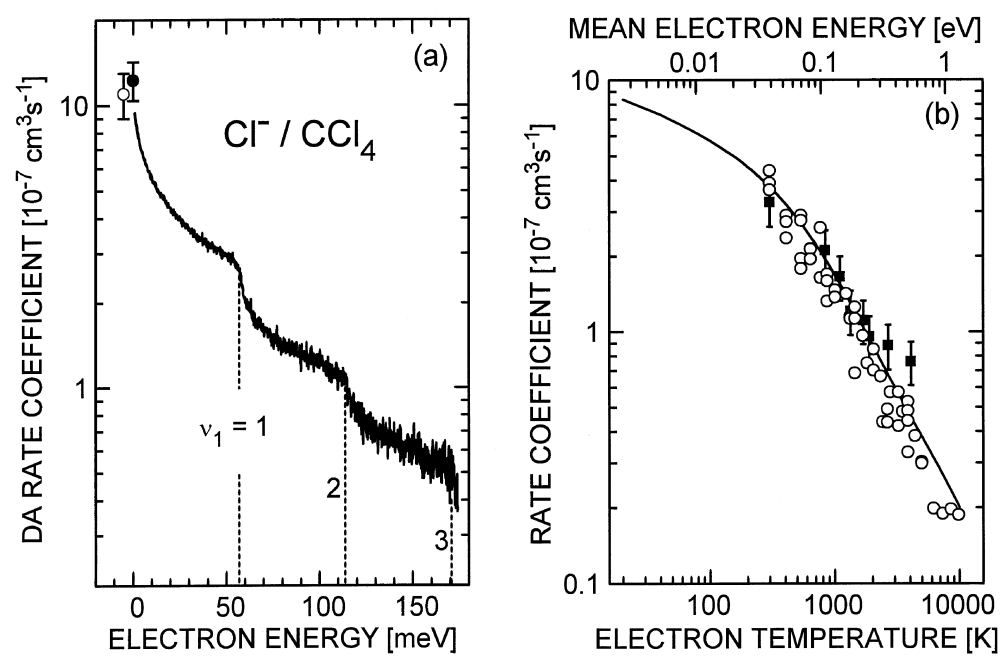

FIG. 33. Dissociative electron attachment to $\mathrm{CCl}_{4}$ molecules: (a) energy dependence of DA rate coefficient $k_{\mathrm{e}}(E)$; (b) thermal rate coefficient $k_{\mathrm{e}}\left(T_{\mathrm{e}}\right)$ for a Maxwellian electron energy distribution and fixed gas temperature $T_{\mathrm{G}}=300 \mathrm{~K}$ (from Klar et al., 2001a). For details see text.

rate coefficient $\mathrm{k}_{n l} \approx 11(2) \times 10^{-7} \mathrm{~cm}^{3} \mathrm{~s}^{-1}$ (open circle, Dunning, 1995; Frey et al., 1995) for RET in $\mathrm{K}^{* *}(n \mathrm{p})+\mathrm{CCl}_{4}$ collisions at high principal quantum numbers $(n \geq 70)$; they are both distinctly higher than the VW capture rate coefficient $k_{\mathrm{c}}(E \rightarrow 0)=6.74 \times 10^{-7} \mathrm{~cm}^{3} \mathrm{~s}^{-1}$, possibly due to the influence of the bound ${ }^{2} \mathrm{~A}_{1}$ anion state (see above). As compared to their appearance in the DA cross-section (Fig. 32), the downward steps at the onsets for excitation of one $\left(v_{1}=1\right)$, two $\left(v_{1}=2\right)$, and three quanta $\left(v_{1}=3\right)$ of the symmetric stretch vibration show up more clearly in the plot of the DA rate coefficient $k_{\mathrm{e}}(E)$. The vertical dashed lines at the three vibrational onsets label the respective vibrational energy positions determined by Raman spectroscopy. So far, no theoretical calculations for DA involving $\mathrm{CCl}_{4}$ are available to our knowledge.

Using the combined DA cross-section shown in Fig. 32, Klar et al. (2001a) calculated thermal DA rate coefficients $k_{\mathrm{e}}\left(T_{\mathrm{e}}\right)$ for fixed gas temperature $T_{\mathrm{G}}=300 \mathrm{~K}$ as a function of electron temperature $T_{\mathrm{e}}$. In Fig. 33b the obtained results (full line) are compared with two sets of swarm data, obtained by Shimamori et al. (1992b) with a microwave cavity pulse radiolysis - microwave heating (MWPR-MH) method (open circles) and by Španel et al. (1995) with a flowing afterglow/Langmuir probe (FALP) apparatus involving an electron swarm with a variable temperature (full squares). We note that the respective rate coefficients at 
$T=T_{\mathrm{e}}=T_{\mathrm{G}}=300 \mathrm{~K}$ agree within their mutual experimental uncertainties (see Klar et al., 2001a). Good overall agreement with regard to the dependence on electron temperature is observed between the calculated results and the swarm data although it appears - as discussed by Klar et al. (2001a) - that the drop in the rate coefficients towards higher temperatures is somewhat slower in the FALP data than in both the LPA derived and in the MWPR-MH results. We emphasize that it is important that the beam data include results obtained with very high resolution in order to avoid uncertainties associated with the true behavior of the attachment crosssection at the lowest energies. For $\mathrm{CCl}_{4}$, about $60 \%$ of the total thermal rate coefficient $k_{\mathrm{e}}\left(T_{\mathrm{e}}=T_{\mathrm{G}}=T=300 \mathrm{~K}\right)$ stem from electrons with energies $E \leq \mathrm{k}_{\mathrm{B}} T$ (Klar et al., 2001a).

\section{B.4. Electron Attachment to $C_{60}$}

The fullerene molecule $\mathrm{C}_{60}$ has astounding electron attachment properties; long-lived $\mathrm{C}_{60}^{-}$anions are formed from near zero to above $10 \mathrm{eV}$ electron energy (Lezius et al., 1993). Here we dwell on the threshold behavior of the cross-section which has been a subject of some controversy. Flowing afterglow/Langmuir probe (FALP) measurements (Smith et al., 1993; Smith and Španel, 1996) indicated that electron capture by $\mathrm{C}_{60}$ is characterized by an activation barrier of $0.26 \mathrm{eV}$. This was interpreted as a p-wave process by Tosatti and Manini (1994) who showed that an s state of the $\mathrm{C}_{60}^{-}$anion is prohibited by symmetry. Their calculations of the capture rates, based on a finite potential well model, are in good agreement with the FALP measurements (Smith et al., 1993; Smith and Španel, 1996), both in terms of the absolute magnitude and the slope of the rate dependence on the inverse electron temperature which gives the magnitude of the activation barrier.

However, as noted previously by Huang et al. (1995), the model used by Tosatti and Manini (1994) and later by Matejcik et al. (1995), does not seem to represent the physics of the process correctly. First, it ignores the polarizability of $\mathrm{C}_{60}$ which is very large (558 a.u.). Furthermore, it regards the capture cross-section as being identical to the elastic cross-section which is physically incorrect. The simplest way to see this is by looking at the threshold behavior: whereas the p-wave capture cross-section behaves as $E^{1 / 2}$ at low energies, the elastic p-wave scattering cross-section is proportional to $E^{2}$. The extra factor $E^{3 / 2}$ in the elastic cross-section appears because the electron has to tunnel through the centrifugal barrier a second time when leaving the interaction zone. Therefore the good agreement between the FALP experiments and the calculations of Tosatti and Manini (1994) seems to be fortuitous. 
Several beam measurements (Jaffke et al., 1994; Huang et al., 1995; Matejcik et al., 1995) also claim the existence of an activation barrier with a height of 0.24 (Jaffke et al., 1994) or $0.15 \mathrm{eV}$ (Huang et al., 1995). The measurements of Jaffke et al. (1994) were reinterpreted by Weber et al. (1996) who concluded that they can only be understood if an s-wave contribution or a resonance close to zero energy are present. Note that the deconvoluted results of Jaffke et al. (1994) were incorrectly shifted on the energy scale by $0.4 \mathrm{eV}$ (see appendix in Weber et al., 1996), thereby suggesting a barrier; this, however, was an artifact of the deconvolution procedure of Jaffke et al. (1994). The presence of the $0.15(5) \mathrm{eV}$ threshold in the experiment of Huang et al. (1995) is not yet understood; in Fig. 7 of Huang et al. (1995) the low-energy peak for $\mathrm{C}_{60}^{-}$formation is found to be shifted by about $+80 \mathrm{meV}$ relative to the peak for $\mathrm{SF}_{6}^{-}$formation.

The absence of an activation barrier is indicated by experiments on Rydberg electron attachment to $\mathrm{C}_{60}$ (Finch et al., 1995; Huang et al., 1995; Weber et al., 1996). They exhibit a flat dependence of the attachment rate on the principal quantum number $n$ of the Rydberg electron at high $n$, indicating an s-wave attachment process. In addition, more recent beam experiments (Elhamidi et al., 1997; Vasil'ev et al., 1997; Kasperovich et al., 2001) with free electrons have found evidence for a zero-energy attachment process (within about $0.03 \mathrm{eV}$ ). Several mechanisms for s-wave attachment involving formation of weakly bound (Weber et al., 1996) or virtual (Lucchese et al., 1999) states of $\mathrm{C}_{60}^{-}$, supported by the long-range polarization interaction, have been discussed.

Here we compare the obtained experimental information with the results of the application of the VW model. In Fig. 10 we presented the $l=0$ through $l=4$ contributions to the cross-sections for a capture by a target with the polarizability 558 a.u., as appropriate for $\mathrm{C}_{60}$ (Bonin and Kresin, 1997). We see that the $E^{1 / 2}$ behavior for the p wave occurs within a very narrow energy range: the p-wave cross-section peaks at $E=26 \mathrm{meV}$. This means that the near-zero energy peaks in the $\mathrm{C}_{60}^{-}$yield, observed in electron beam experiments by several groups, could - at least in part - reflect such a p-wave contribution.

Recent ab initio theoretical calculations (Gianturco et al., 1999; Lucchese et al., 1999) of elastic e- $\mathrm{C}_{60}$ scattering suggest that in the low-energy region this process is dominated by a virtual state in the $\mathrm{a}_{\mathrm{g}}$ symmetry, whose lowest partial wave component is $l=0$, and a resonance state in the $\mathrm{t}_{1 u}$ symmetry whose lowest partial-wave component is $l=1$. Therefore one may assume that the attachment process at low energies is controlled by a combination of direct capture mediated by a virtual state (similar to low-energy attachment to $\mathrm{SF}_{6}$ ) and resonance capture into the $\mathrm{t}_{1 u}$ state. In model calculations of the threshold behaviour for $\mathrm{C}_{60}^{-}$formation in RET and free 
electron attachment to $\mathrm{C}_{60}$, Fabrikant and Hotop (2001) correspondingly described the capture cross-section by combining the s-wave and the p-wave VW cross-sections, $\sigma_{0}$ and $\sigma_{1}$ :

$$
\sigma(E)=c\left(\varepsilon \sigma_{0}+\sigma_{1}\right)
$$

where $c$ and $\varepsilon$ are adjustable parameters: $\varepsilon$ characterizes the relative contribution of the s-wave and $c$ the absolute value of the cross-section which was estimated from experimental RET attachment rate coefficients (Finch et al., 1995; Huang et al., 1995; Weber et al., 1996). A combined fit to these $n$-dependent RET data and to the free electron data of Elhamidi et al. (1997) yielded $c=0.1$ and $\varepsilon=0.1-0.2$ (Fabrikant and Hotop, 2001). The s-wave zero energy peak dominates the cross-section only at very low energies (below $3 \mathrm{meV}$ ). High-resolution free electron attachment experiments are necessary to confirm this prediction.

Note that only $1-2 \%$ ( $c \varepsilon=0.01$ or 0.02$)$ of the s-wave VW cross-section appears to contribute to the capture process, in contrast with attachment to $\mathrm{SF}_{6}$. Apparently the stabilization mechanism discussed for $\mathrm{SF}_{6}$ is not as efficient for $\mathrm{C}_{60}$. The $1-2 \%$ fraction can be considered as an efficiency for conversion of the $\mathrm{C}_{60}^{-}$virtual state into a bound state, something similar to the survival probability in the resonance attachment. It has to be mentioned that the temperature dependence of the attachment rate coefficient observed in the FALP experiments (Smith et al., 1993; Smith and Španel, 1996) was found to be not in accord with the combined s- and p-wave model. Possibly, the electron energy distribution in the temperature-variable FALP apparatus are not completely thermalized and short of low-energy electrons. Another possibility is a significant dependence of the negative ion yield on the rovibrational temperature of $\mathrm{C}_{60}$ (Fabrikant and Hotop, 2001). At higher temperatures many nontotally symmetric vibrations are excited, and s-wave attachment might play a more substantial role (Vasil'ev et al., 2001).

\section{B.5. Electron Scattering from $\mathrm{CO}_{2}, \mathrm{CS}_{2}$, and $\mathrm{N}_{2} \mathrm{O}$}

\section{5.a. Carbon Dioxide}

Carbon dioxide is linear in its electronic ground state. The lowest vibrationally excited states, labelled as $\left(v_{1} v_{2} v_{3}\right)$, include the bending mode (010) $(82.7 \mathrm{meV})$, the Fermi-coupled pair of the bending overtone and the symmetric stretch mode $(020) /(100)(159.4 / 172.1 \mathrm{meV}$, average energy $165.7 \mathrm{meV})$, the Fermi-coupled pair $(030) /(110)(239.6 / 257.5 \mathrm{meV}$; average energy $248.3 \mathrm{meV})$, the asymmetric stretch mode (001) $(291.3 \mathrm{meV})$, and the Fermi-coupled triplet $(040) /(120) /(200)(317 / 342 / 348 \mathrm{meV}$, average energy 
$336 \mathrm{meV}$ ) (Herzberg, 1945). $\mathrm{CO}_{2}^{-}$has long been known to have a bent equilibrium configuration, generally made responsible for the mass spectrometric observation of metastable $\mathrm{CO}_{2}^{-}$anions. In recent ab initio work, Gutsev et al. (1998) obtained a bond angle of $137.8^{\circ}$, an electric dipole moment of $-0.90 \mathrm{D}$ and a negative adiabatic electron affinity of $E A_{\text {ad }}\left(\mathrm{CO}_{2}\right)=-0.66 \mathrm{eV}$ for this state of the anion.

Vibrational excitation in $\mathrm{CO}_{2}$ has two distinct energy ranges. The first is around the ${ }^{2} \Pi_{\mathrm{u}}$ shape resonance at $3.6 \mathrm{eV}$, where boomerang structures have long been known (Boness and Schulz, 1974). The second is dominated by a virtual state below $2 \mathrm{eV}$ (Morrison, 1982; Herzenberg, 1984; Estrada and Domcke, 1985; Kochem et al., 1985; Morgan, 1998; Rescigno et al., 1999; Mazevet et al., 2001; Field et al., 2001b). The virtual state causes dramatic enhancement of the cross-section near threshold for the excitation of the symmetric stretch vibration (100), Fermi-coupled with two quanta of the bending vibration $\left(02^{0} 0\right)$ (Kochem et al., 1985; Field et al., 1991b; Allan, 2001a). The cross-sections for the excitation of the infrared active fundamental vibrations, (010) and (001), also exhibit threshold peaks, but these can be ascribed, at least to a large degree, to direct dipole scattering (Kochem et al., 1985). Pronounced selectivity was observed in the excitation of the Fermi-coupled vibrations $\left\{\left(10^{0} 0\right),\left(02^{0} 0\right)\right\}$ in the virtual state range (Antoni et al., 1986; Allan, 2001a). Selectivity in the excitation of the Fermi-coupled vibrations was also observed in the shape resonance region (Johnstone et al., 1995; Kitajima et al., 2000; Allan, 2001a), but this energy range does not fall into the scope of the present review.

In contrast to the halogen hydrides the elastic cross-section and the crosssections for the excitation of the fundamental vibrations do not exhibit structures of vibrational origin (Kochem et al., 1985; Field et al., 1991b). This corresponds to the expectation, reflecting the fact that the cross-section enhancement at threshold is due to a virtual state, which is not associated with a time delay (Herzenberg, 1984), and consequently cannot support vibrational structure. Tennysson and Morgan (1999) predicted, however, on theoretical grounds, that the virtual state becomes bound in the electronic sense upon sufficiently strong bending of the $\mathrm{CO}_{2}$.

This idea has received strong support from the experimental observation of vibrational Feshbach resonances and boomerang oscillations in the excitation of certain higher-lying Fermi-coupled vibrations involving symmetric stretch and bending by Allan (2002a), as shown in Fig. 34. Allan (2002a) discussed two possible explanations of these structures. The first, considered less probable, is that these structures are due to metastable vibrational levels around the minimum of the valence ground state of $\mathrm{CO}_{2}^{-}$ at a bend geometry, that is that they correspond to the outer well resonances discussed in this review already in connection with $\mathrm{HCl}$. The second 


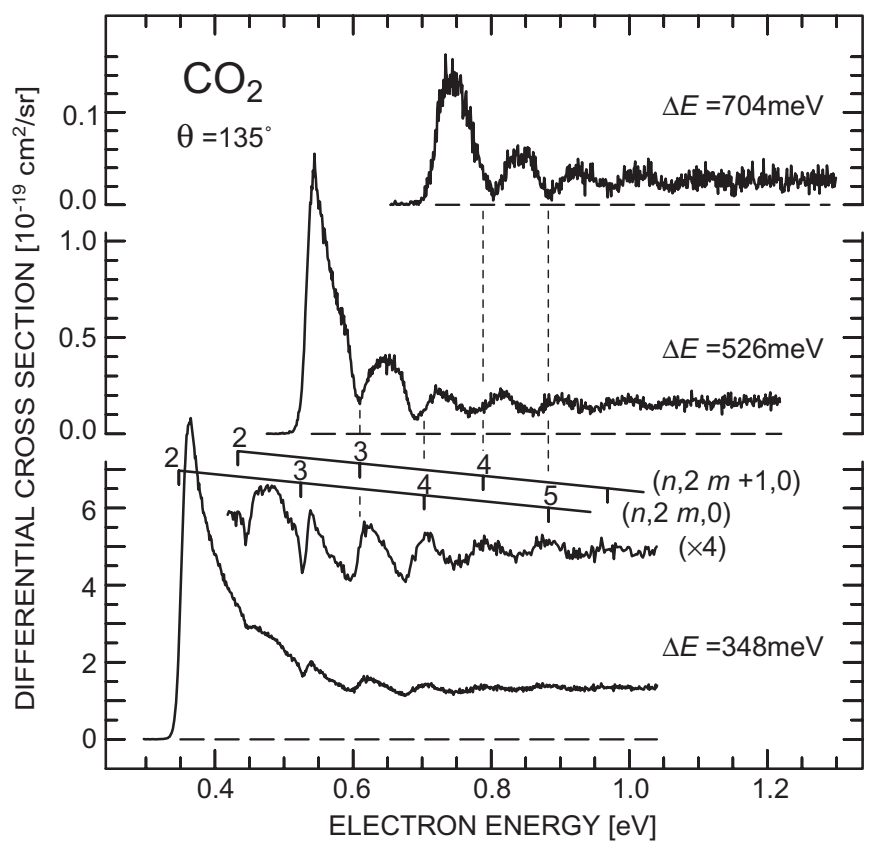

FIG. 34. Cross-sections for exciting vibrational overtones in $\mathrm{CO}_{2}$, specifically the highestenergy components of the Fermi polyads $\left\{\left(n, 2 m^{0}, 0\right)\right\}$ where $n$ and $m$ are zero or positive integers such that (from bottom to top) $n+m=2,3$, and 4 . (i.e., $\left\{\left(n, 2 m^{0}, 0\right)\right\}, n+m=3$, stands for four Fermi-coupled states resulting from Fermi-mixing of the first order states $\left(3,0^{0}, 0\right)$, $\left(2,2^{0}, 0\right),\left(1,4^{0}, 0\right),\left(0,6^{0}, 0\right)$.) The grids above the bottom spectrum show the thresholds for exciting the highest-energy components of the polayds $\left\{\left(n, 2 m^{0}, 0\right)\right\}$ and $\left\{\left(n, 2 m+1^{1}, 0\right)\right\}$, with the sums $n+$ m given (from Allan, 2002a).

explanation, considered more probable, is that the structures are due to 'inner well' vibrational Feshbach resonances and boomerang oscillations supported by a potential surface of the $\mathrm{CO}_{2}^{-}$where an electron is weakly electronically bound by dipole and polarization force in the bent and stretched geometry. The wave function of the electron is spatially very diffuse. This 'diffuse' state of $\mathrm{CO}_{2}^{-}$becomes the (unbound) virtual state at the linear geometry.

The latter explanation has received strong support from the $\mathrm{CO}_{2}^{-}$ potential surface calculated by Sommerfeld (2003). His elaborate calculation succeeded in reproducing both the valence and the diffuse states of $\mathrm{CO}_{2}^{-}$ within the same model. The cut through the adiabatic surface along the bending coordinate, shown in Fig. 35, shows that the valence ground state of $\mathrm{CO}_{2}^{-}$, which has a shallow minimum at bent geometries, rapidly acquires 


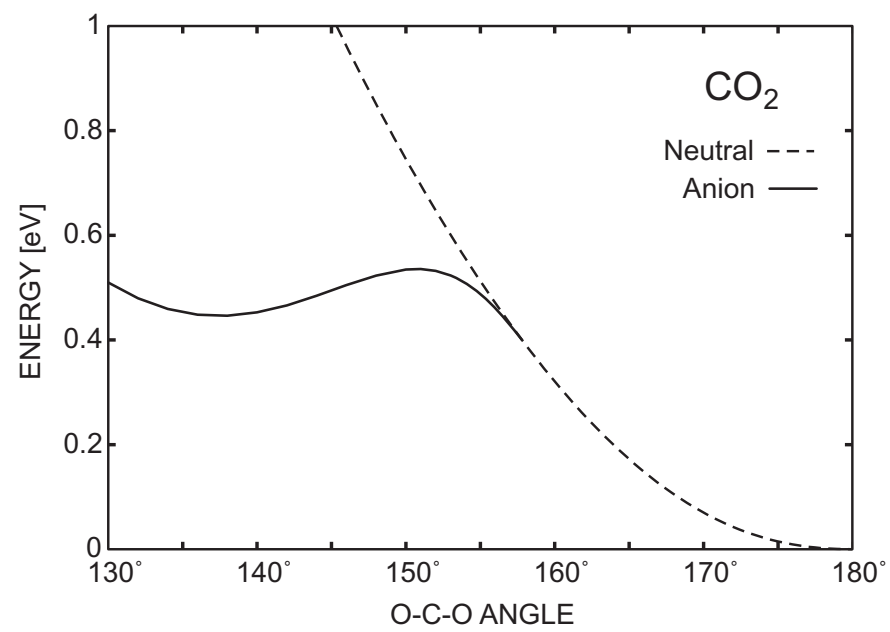

FIG. 35. Adiabatic potential curves for $\mathrm{CO}_{2}$ (dashed) and $\mathrm{CO}_{2}^{-}$(solid line). The electronic wave function of $\mathrm{CO}_{2}^{-}$is valence-like for $\mathrm{O}-\mathrm{C}-\mathrm{O}$ angles below about $150^{\circ}$, where it forms the outer well. It sharply bends down and becomes diffuse between about 150 and $160^{\circ}$. It disappears (becomes a resonance and finally a virtual state) at angles above about $160^{\circ}$ (Sommerfeld, 2003).

diffuse character and turns sharply down as the $\mathrm{CO}_{2}$ framework is straightened towards the linear geometry. Below a certain bending angle the anion and neutral surfaces cross and the anionic surface disappears in the adiabatic sense. The situation is closely related to that discussed in Section II in connection with Fig. 6. Qualitatively, the oscillations of the nuclear wave packet on the 'inner well' of the $\mathrm{CO}_{2}^{-}$potential surface can be pictured in the same way as already discussed for $\mathrm{HF}^{-}$. Around the linear geometry the $\mathrm{CO}_{2}^{-}$starts to loose the extra electron since it is no longer bound in the adiabatic sense. The electron departs only slowly, however, since we are near threshold. Part of the electron wave function is recaptured when the nuclei swing over to bent geometry again, giving rise to VFRs of varying width. The similarity between the $\mathrm{HF}$ and $\mathrm{CO}_{2}$ cases is born out by the striking phenomenological similarity of the $\mathrm{HF}$ and $\mathrm{CO}_{2}$ spectra (Figs. 18 and 34).

It is interesting to compare the present structures to those reported by Leber et al. (2000b) in dissociative electron attachment to $\mathrm{CO}_{2}$ clusters (see also Section IV.C). They observed structures below the (010) and the $\left\{\left(1,0^{0} 0\right),\left(02^{0} 0\right)\right\}$ thresholds, with the difference that a structure was observed for each member of the latter Fermi dyad in their spectra, whereas in the present study a structure is observed only for the topmost members of the Fermi polyads. 


\section{5.b. Carbon Disulfide}

$\mathrm{CS}_{2}$ is isovalent with $\mathrm{CO}_{2}$. Like $\mathrm{CO}_{2}$ it does not have a permanent dipole moment, and it possesses - like $\mathrm{CO}_{2}(Q=-3.1$ a.u. $)$ - a sizeable permanent quadrupole moment $\left(Q\left(\mathrm{CS}_{2}\right)=+3.3\right.$ a.u.) (Compton and Hammer, 2001). It differs from $\mathrm{CO}_{2}$ in several important aspects, however. It is much more polarizable than $\mathrm{CO}_{2}$ (average polarizability $59.4 \mathrm{a}_{0}{ }^{3}$ for $\mathrm{CS}_{2}$ and $19.4 \mathrm{a}_{0}{ }^{3}$ for $\mathrm{CO}_{2}$, Lide, 1995) and has a positive adiabatic electron affinity associated with the bent valence ground electronic state of $\mathrm{CS}_{2}^{-}$(Gutsev et al., 1998), see also Section IV.C.5.

Sohn et al. (1987) measured absolute differential cross-sections in $\mathrm{CS}_{2}$ at a number of discrete energies between 0.3 and $5 \mathrm{eV}$. Allan (2001c) reported deep narrow structures in the elastic and vibrationally inelastic crosssections in a crossed-beam study. Jones et al. (2002) found deep structures in their very low-energy and very high-resolution measurements of the total integral and backward cross-sections using a synchrotron radiation photoelectron source. Jones et al. interpreted them in terms of giant resonances and symmetry selection rules. The total and the differential cross-sections (the latter multiplied by $4 \pi$ to obtain an estimate of the integral cross-section) are compared in Fig. 36. There is a striking agreement of the shapes of the resonant structures. The $30 \%$ difference of the magnitudes could be in part of instrumental origin, in part due to an anisotropy of the differential cross-section. Figure 37 shows the crossed-beam

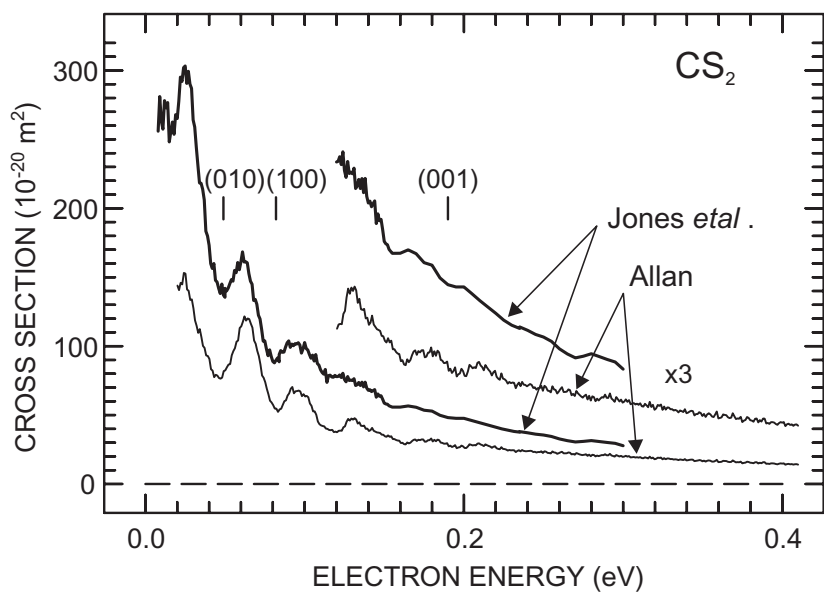

Fig. 36. Comparison of the total cross-section of Jones et al. (2002) with the $\theta=135^{\circ}$ differential elastic cross-section of Allan (2003). The latter was multiplied by $4 \pi$ to obtain an estimate of the integral cross-section. 


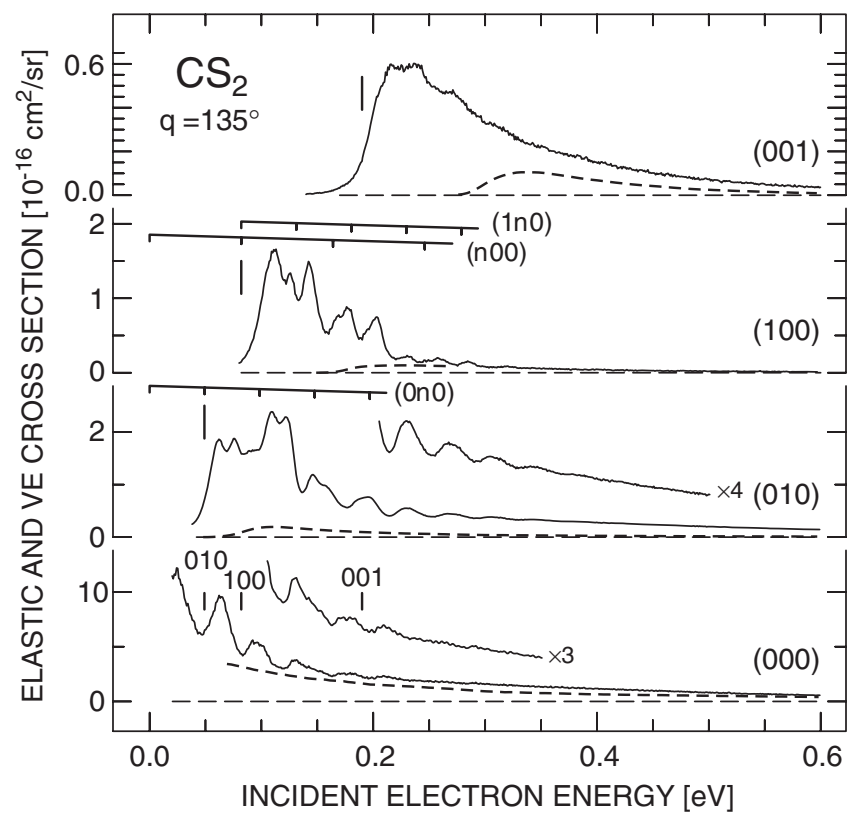

FIG. 37. Differential elastic and vibrationally inelastic cross-sections in $\mathrm{CS}_{2}$ (Allan, 2003). $\mathrm{CO}_{2}$ cross-sections (Allan, 2001a and 2002a,b) are shown for comparison as dashed curves. Vibrational thresholds are indicated by vertical bars above the elastic and to the left of the inelastic cross sections. Grids indicate selected progressions of vibrational energies of neutral $\mathrm{CS}_{2}$.

results of Allan (2001c, 2003) for the elastic cross-section and the excitation of the fundamental vibrations. It shows that near-threshold the elastic, and to an even more dramatic degree the inelastic cross-sections, are larger in $\mathrm{CS}_{2}$ than in $\mathrm{CO}_{2}$. In addition, the elastic and the (010) and (100) crosssections have deep narrow structures, also in contrast to $\mathrm{CO}_{2}$, where the threshold peaks in the cross-sections for the fundamental vibrations are structureless and only certain overtone cross-sections exhibit structure (Fig. 34). The structure in the (001) cross-section is much less pronounced like in $\mathrm{CO}_{2}$.

The structures in the cross-sections are undoubtedly caused by vibrational activity in the negative ion. In contrast to $\mathrm{CO}_{2}^{-}$, it is much harder to assign this activity to a certain electronic state of the anion. The vertical electronic affinity, corresponding to the lowest valence state ${ }^{2} \Pi_{u}$ of $\mathrm{CS}_{2}^{-}$in its linear geometry, is around $0 \mathrm{eV}$ (Gutsev et al., 1998). This state splits into the ${ }^{2} \mathrm{~A}_{1}$ and ${ }^{2} \mathrm{~B}_{2}$ branches upon bending. The minimum of the lower branch corresponds to the adiabatic electron affinity, the experimental and 
theoretical results for which range between about +0.3 and $+0.9 \mathrm{eV}$ (Gutsev et al., 1998). This means that the vibrational levels on both branches of the ${ }^{2} \Pi_{u}$ state of $\mathrm{CS}_{2}^{-}$lie in the same energy range as the structures in the cross-sections and could be responsible for them, apart from the 'diffuse' state which has been invoked to explain the structure in $\mathrm{CO}_{2}$.

The vibrational grids in Fig. 37 indicate that the structures do not, in contrast to $\mathrm{CO}_{2}$, correspond to vibrational thresholds in a consistent manner. It is also impossible to assign the observed structures to vibrational levels of the valence electronic states of $\mathrm{CS}_{2}^{-}$in a convincing manner. These vibrations have been calculated at a very high degree of sophistication by Rosmus and Hochlaf (2002). An assignment is impossible, however, because the calculated density of $\mathrm{CS}_{2}^{-}$vibrational states is very high in this energy range, two branches of the ${ }^{2} \Pi_{u}$ state are present, the vibrational origins of which are not exactly known, and because of further complications due to spin-orbit splitting and the pronounced anharmonicity of the potentials (Rosmus and Hochlaf, 2002).

In addition, $\mathrm{CS}_{2}$ is likely, with its larger polarizability, to exert a higher polarization attraction to an extra electron than $\mathrm{CO}_{2}$. A diffuse electronic state was postulated experimentally and theoretically already in the less polarizable $\mathrm{CO}_{2}$, and it is thus very likely that $\mathrm{CS}_{2}$ also supports a diffuse state, presumably over an even larger range of geometries of the nuclei than $\mathrm{CO}_{2}$. Avoided crossings, similar to those found in $\mathrm{CO}_{2}$ (Fig. 35), are likely to cause a complicated adiabatic potential surfaces of the ground and low-lying excited states of $\mathrm{CS}_{2}^{-}$. This is probably the reason why the structures in the $\mathrm{CS}_{2}$ cross-sections, unlike $\mathrm{CO}_{2}$ (Fig. 34), cannot be convincingly associated with vibrational thresholds. An exception is the total cross-section, very similar to the elastic cross-section, where the dips (not peaks) could be associated with the thresholds for the fundamental vibrations by Jones et al. (2002). The remaining sharp structures, at least in the vibrationally inelastic cross-sections, lack detailed explanation at present.

\section{5.c. Nitrous Oxide}

$\mathrm{N}_{2} \mathrm{O}$ is isoelectronic with $\mathrm{CO}_{2}$. Their (average) polarizabilities $\left(\alpha\left(\mathrm{N}_{2} \mathrm{O}\right)=\right.$ $20.4 \mathrm{a}_{0}{ }^{3}$, Lide, 1995) agree to within $5 \% . \mathrm{N}_{2} \mathrm{O}$ differs from $\mathrm{CO}_{2}$ in that it has a small permanent dipole moment (0.16 Debye, Lide, 1995) and, more importantly, in the much lower lying threshold for dissociative electron attachment. Cross sections above about $1 \mathrm{eV}$ have been studied both experimentally and theoretically (see for example Winstead and McKoy, 1998, and the experimental work cited therein). Allan and Skalický (2003) recently measured the cross-sections below $1 \mathrm{eV}$, in an energy range with which this review is concerned. 


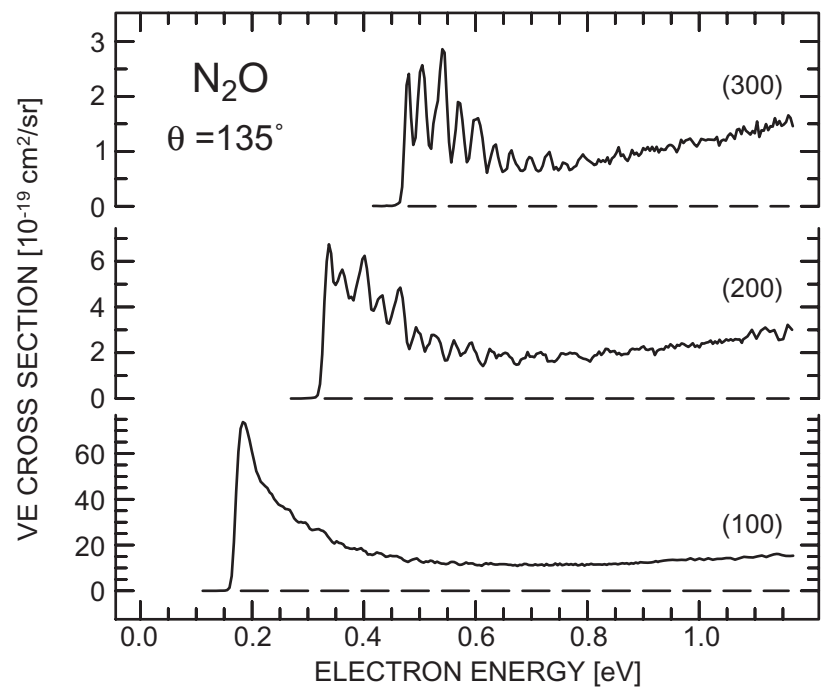

FIG. 38. Differential cross-sections for exciting the symmetric stretch vibration in $\mathrm{N}_{2} \mathrm{O}$ and its overtones (from Allan and Skalický, 2003).

The vibrationally inelastic cross-sections shown in Fig. 38 reveal a close phenomenological similarity with $\mathrm{CO}_{2}$. The cross-section for the excitation of the fundamental vibrations are structureless (or nearly so) in both $\mathrm{N}_{2} \mathrm{O}$ and $\mathrm{CO}_{2}$, but structures appear in the excitation of overtones. The structure in $\mathrm{N}_{2} \mathrm{O}$ is richer and deeper than in $\mathrm{CO}_{2}$. In analogy with $\mathrm{CO}_{2}$ we assign it to vibrational Feshbach resonances supported by an electronic state of $\mathrm{N}_{2} \mathrm{O}^{-}$with a spatially diffuse electron wave function.

The low threshold to dissociative electron attachment permits the study of the vibrational Feshbach resonances in the dissociative channel, as shown in Fig. 39. The spectra were recorded with a resolution of about $10 \mathrm{meV}$ in the incident beam, using an electron spectrometer with hemispherical analysers and a Wien filter to separate electrons and ions (Fig. 13). For energetic reasons, the ions can only be $\mathrm{O}^{-}$. The spectrum on the left resembles closely the spectra of Chantry (1969), Brüning et al. (1998) and Krishnakumar and Srivastava (1990). The detail of the spectrum on the right reveals that what initially appeared as a continuous band consists in reality of narrow peaks (vibrational Feshbach resonances VFR), whose spacings and positions are related to the bending vibration of $\mathrm{N}_{2} \mathrm{O}$. It thus appears that the mechanism of dissociative electron attachment in $\mathrm{N}_{2} \mathrm{O}$ is more complex than the simple picture presented in Fig. 2 in the introduction. The VFRs act as "doorway states" and then predissociate into 

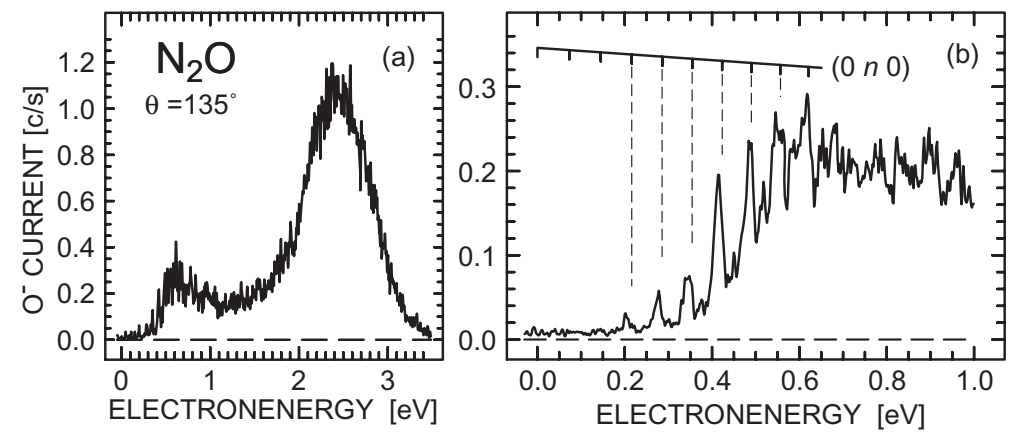

FIG. 39. Dissociative electron attachment spectrum in $\mathrm{N}_{2} \mathrm{O}$. A wider energy range is shown at left (a), detail at low energies at right (b) (from Allan and Skalický, 2003).

the dissociative continuum. This mechanism is analogous to that invoked theoretically for $\mathrm{CH}_{3} \mathrm{Cl}$ in connection with Fig. 20. Experimentally, it has been invoked previously in the strongly polar molecule ethylene carbonate (Stepanović et al., 1999), although the individual VFRs were not resolved there. Sommerfeld (2002) discussed the role of VFRs as doorway states from the theoretical point of view in nitromethane. $\mathrm{N}_{2} \mathrm{O}$ represents a particularly convincing case because the individual VFRs could be resolved.

These results shed new light on the astounding observation of extremely narrow peaks (with widths down to $2.3 \mathrm{meV}$ ) in the laser photoelectron attachment spectra of $\mathrm{N}_{2} \mathrm{O}$ clusters yielding both heterogeneous $\left(\mathrm{N}_{2} \mathrm{O}\right)_{q} \mathrm{O}^{-}$ and homogeneous $\left(\mathrm{N}_{2} \mathrm{O}\right)_{q}^{-}$cluster anions (Weber et al., 1999a; Leber et al., 2000c). These peaks were found to be located just below the onsets for vibrational modes of the free $\mathrm{N}_{2} \mathrm{O}$ molecule (redshifts in the meV range) and interpreted as VFRs. Similar VFRs with substantially larger redshifts were subsequently found in attachment spectra for $\mathrm{CO}_{2}$ cluster anions (Leber et al., 2000b; Barsotti et al., 2002a) as well as for OCS cluster anions. Evidence for VFRs in $\mathrm{O}_{2}$ cluster anion production has been obtained by Matejcik et al. (1996, 1999). See Section IV.C for further discussion.

\section{Electron Attachment to Molecular Clusters}

In this section we discuss resonance phenomena in low-energy electron attachment to molecular clusters, yielding stable or long-lived anions detected by mass spectrometers. The reaction scheme can be characterized 
as follows $(\{\alpha\}$ denotes a set of quantum numbers for the electronic and ro-vibrational states in question):

$$
\begin{aligned}
(X Y)_{N}(\alpha)+\mathrm{e}^{-}(E) \rightarrow(X Y)_{N}^{-*} \rightarrow & (X Y)_{N}\left\{\alpha^{\prime}\right\}+\mathrm{e}^{-}\left(E^{\prime}\right) \\
\rightarrow & (X Y)_{q-1} Y^{-}+X+(N-q) X Y \\
& (q \leq N) \\
\rightarrow & (X Y)_{q}^{-}+(N-q) X Y \quad(q \leq N 1) \\
\rightarrow & (X Y)_{N}^{-\dagger} \quad(\tau>100 \mu \mathrm{s})
\end{aligned}
$$

Here path (42a) describes elastic $\left(\alpha=\alpha^{\prime}\right)$ or inelastic scattering. Process (42b) represents dissociative electron attachment (DA) while in the reaction (42c) (only relevant for clusters, i.e. $N \geq 2$ ) anion formation proceeds through evaporation of $X Y$ constituents. Even if no other stabilizing process occurs, the temporary negative ion $(X Y)_{N}^{-*}(N \geq 1)$ can become metastable with respect to spontaneous re-emission (autodetachment) of the electron, if the electronic energy is rapidly redistributed into internal degrees of freedom (intra- and intermolecular vibration, rotation), thereby yielding long-lived negative ions $(X Y)_{N}{ }^{-*}$ in path (42d), e.g. $\mathrm{SF}_{6}^{\ddagger}$ from $\mathrm{SF}_{6}$ (see section IV.B) or small $\left(\mathrm{H}_{2} \mathrm{O}\right)_{N}^{-*}$ anions $(N=2,6,7)$ (Weber et al., 1999b).

Beams of molecular clusters are typically created in supersonic beams (often using molecules as a minority seed gas in a rare gas atom carrier gas). Thus a broad size distribution of molecular clusters is produced. The correlation of the observed anion cluster size $q$ with the size $N$ of the neutral cluster precursor relevant for the reactions (42) is thus not a priori known. Positive cluster ion mass spectra, induced by electron impact at typically $70-100 \mathrm{eV}$ electron energy, help to diagnose the presence of neutral clusters, but normally do not directly reflect the neutral cluster size distribution due to fragmentation effects which may be very strong for weakly bound molecular clusters (Buck, 1988). Comparisons of the mass spectra for positive and negative cluster ions due to electron impact ionization and electron attachment, respectively, are nevertheless useful in that peculiarities of anion formation become visible in a rather direct, albeit qualitative way. Schemes for size selection of neutral cluster beams, based on scattering from atomic beams, have been developed (Buck and Meyer, 1986), but they yield rather low size-selected target densities. To our knowledge, size-selected targets of molecular clusters have not been used in electron attachment work so far.

One of the interesting features of clusters is their role as nanoscale prototypes for studying the effects of solvation on the characteristics of both solvent and solvated particle, due to the interaction between a solvated molecule or ion and its surrounding solvent environment. Solvation effects 
also play a key role in the formation of negative ions by attachment of slow electrons to clusters. The investigation of cluster anion formation in collisions of energy-controlled electrons with molecular clusters was pioneered by the Oak Ridge group (Klots and Compton, 1977, 1978) and subsequently studied by groups in Konstanz (Knapp et al., 1985, 1986a, b, 1987), Innsbruck (Märk et al., 1985, 1986; Stamatovic et al., 1985a, b; Märk, 1991; Rauth et al., 1992; Matejcik et al., 1996, 1999), and Berlin (Hashemi et al., 1990, 1991, 1992; Illenberger, 1992, 2000; Jaffke et al., 1992; Ingolfsson et al., 1996); typical electron energy widths ranged from 0.1 to $1 \mathrm{eV}$. It was shown that the repulsive negative ion resonances which dominate anion formation for the monomer molecules via dissociative attachment at energies in the range $1-10 \mathrm{eV}$ are also important for cluster anion formation. In clusters, the resonances appear shifted towards lower energies due to the effects of solvation. For molecular clusters $(X Y)_{N}$ additional features may be observed which reflect the effects of the cluster environment on the resonance energy and symmetry (Compton, 1980; Märk, 1991; Ingolfsson et al., 1996). One aspect is the appearance of anion resonances in clusters whose formation is symmetry-forbidden for the isolated molecule (Märk, 1991; Illenberger, 1992). Another intriguing result in cluster anion formation is the observation of a prominent resonance at zero energy (indicative of an s-wave attachment process) in cases where such a feature is absent in the monomer (Märk et al., 1985; Stamatović, 1988; Märk, 1991). The first observations of these 'zero energy resonances' for clusters of oxygen (Märk et al., 1985), carbon dioxide (Stamatovic et al., 1985a, b; Knapp et al., 1985, 1986a) and water (Knapp et al., 1986b, 1987) were made by groups at Innsbruck and Konstanz at too broad energy widths $(0.5-1.0 \mathrm{eV})$, as to elucidate possible structure (e.g. due to vibrational effects) and to thoroughly understand the origin of these zero energy peaks.

Anion formation in collisions of molecular clusters with near-zero energy electrons can also be studied by Rydberg electron transfer (RET), as initiated by Kondow et al. (Kondow 1987), who used electron impact to produce $\mathrm{Kr}^{* *}(n)$ Rydberg atoms with a band of principal quantum numbers $n$ around 25. RET to molecular clusters with state-selected Rydberg atoms was pioneered by groups at Kaiserslautern (Kraft et al., 1989) and Villetaneuse (Desfrançois et al., 1989). Interesting structure was observed in the size dependence of several RET-induced anion cluster mass spectra such as those for $\left(\mathrm{CO}_{2}\right)_{q}^{-}$(Kondow, 1987; Kraft et al., 1989) and $\left(\mathrm{N}_{2} \mathrm{O}\right)_{q} \mathrm{O}^{-}$ formation (Kraft et al., 1990), but the origin remained unclear. Rather sharp peaks in the $n$-dependence of the RET yield for anion formation involving strongly dipolar molecules and clusters were observed by the Villetaneuse group (Desfrançois et al., 1994a, b,c) and interpreted as being due to a rather $n$-selective curve-crossing mechanism. In combination with studies of 
field detachment, valuable information on binding energies of electrons, weakly bound to molecules and clusters by long-range forces, was derived (Desfrançois et al., 1996, Compton and Hammer, 2001).

In the following subsections we shall discuss free electron attachment to homogeneous molecular clusters in the range of the 'zero energy resonance', carried out with sufficiently high resolution to resolve vibrational structure. To put the results for clusters into a proper perspective we include brief discussions of the characteristics for electron scattering from the respective monomer molecule. Although not covered in detail here, we mention that in mixed clusters (i.e. either molecule-atom or molecule-molecule clusters) interesting features in the energy dependence for anion formation have been observed which occur close to thresholds for sufficiently strong inelastic processes in one of the constituents (Illenberger, 1992; Rauth et al., 1992). The underlying process is addressed as 'autoscavenging' (Rauth et al., 1992): as a result of the inelastic event the scattered electron propagates as a near-zero energy electron which may be efficiently captured within the cluster if the other constituent has a prominent zero energy resonance. Autoscavenging can also occur in homogeneous clusters if the constituents exhibit both a prominent zero energy resonance and a strong inelastic channel at higher energies. It was discovered by Klots and Compton (1980) for the case of methyl iodide clusters and addressed as 'self-scavenging'. Both autoscavenging and self-scavenging should not be confused with true resonances (such as vibrational Feshbach resonances) of the electron-cluster system.

\section{C.1. Oxygen Clusters}

Electron scattering from oxygen molecules at energies below $1 \mathrm{eV}$ is strongly influenced by the well-known $\mathrm{O}_{2}^{-}\left({ }^{2} \Pi_{\mathrm{g}}\right)$ resonance (Schulz, 1973b; Allan, 1995). Addition of a $\pi_{\mathrm{g}}$ electron to the oxygen molecule yields a stable negative ion with an adiabatic binding energy of $0.451(7) \mathrm{eV}$ (Travers et al., 1989 ) and an equilibrium distance about $12 \%$ longer than that of neutral $\mathrm{O}_{2}$. The four lowest vibrational levels $v^{\prime}=0-3$ of the $\mathrm{O}_{2}^{-}\left({ }^{2} \Pi_{\mathrm{g}}, v^{\prime}\right)$ anion are truly bound with a vibrational quantum of $\Delta G_{01}=134.4(8) \mathrm{meV}$ (Bailey et al., 1996). The center-of-gravity of the fine-structure split $v^{\prime}=4$ anion state is located $0.09 \mathrm{eV}$ above the neutral ground state of $\mathrm{O}_{2}\left(\mathrm{X}, v^{\prime \prime}=0\right)$ (Land and Raith, 1974), and the states with $v^{\prime} \geq 4$ are thus subject to autodetachment with decay widths $\Gamma_{v^{\prime}}(E)$, rising strongly $\left(\propto E^{5 / 2}\right)$ with electron energy $E$, as characteristic for a $\mathrm{d} \pi_{\mathrm{g}}$-wave shape resonance for the electron- $\mathrm{O}_{2}$ system; for $v^{\prime}=6$, the width is about $1 \mathrm{meV}$ (Field et al., 1988; Allan, 1995; Higgins et al., 1995). Correspondingly, the formation of 
long-lived oxygen anions in electron collisions at sub-eV energies requires rapid collisional stabilization of the temporary $\mathrm{O}_{2}^{-}$anion, as possible in high- pressure media (Bloch and Bradbury, 1935; Christophorou, 1978; Hatano and Shimamori, 1981) or for oxygen bound in aggregates (homogeneous or heterogeneous clusters) (Märk, 1991; Illenberger, 1992; Hatano, 1997). The built-in stabilization capability of clusters was, e.g., demonstrated in a recent RET study involving oxygen monomers and dimers (Kreil et al., 1998): while $\mathrm{O}_{2}^{-}$formation from $\mathrm{O}_{2}$ molecules is very inefficient (and only possible through efficient postattachment interactions with the Rydberg ion core) (Walter et al., 1986; Harth et al., 1989) the rate coefficient for $\mathrm{O}_{2}^{-}$formation from oxygen dimers was found to exceed that involving monomers by four orders of magnitude (Kreil et al., 1998).

In free electron attachment to oxygen clusters at low energies, only homogeneous $\left(\mathrm{O}_{2}\right)_{q}^{-}$anions $(q \geq 1)$ can be formed. In the yield for anions with $q=1,2,10$, measured with about $0.5 \mathrm{eV}$ electron energy width, Märk et al. (1985) found a prominent peak near zero eV; for large $q$, the width of this peak was narrower than for $q=1$ and 2. Later, Illenberger and coworkers studied $\left(\mathrm{O}_{2}\right)_{q}^{-}(q=1-4)$ anion formation from oxygen clusters with an energy-selected electron beam of about $0.2 \mathrm{eV}$ width (Hashemi et al., 1991, 1992; Jaffke et al., 1992); they detected the maximum respective anion yield at energies clearly above zero energy. More recently, the Innsbruck group (Matejcik et al., 1996, 1999) had a closer look at anion formation from oxygen clusters, using an energy-selected, magnetically-collimated electron beam with a stated energy width around $0.03 \mathrm{eV}$. They reported the yield for small cluster anions $\left(\mathrm{O}_{2}\right)_{q}^{-}(q=1,2,3)$ over the energy range 0 $1 \mathrm{eV}$. Apart from a resolution-limited peak near-zero energy they detected peak structure starting at about $80 \mathrm{meV}$ with spacings around $110 \mathrm{meV}$ and superimposed on the general drop of the attachment yield towards higher energies. This structure was ascribed to vibrational levels of the oxygen anion solvated in oxygen molecules (Matejcik et al., 1999).

In contrast to the findings of the Innsbruck group for $\left(\mathrm{O}_{2}\right)_{q}^{-}$formation $(q=1-3)$, no clear peak structure is observed in the attachment spectra for $\left(\mathrm{O}_{2}\right)_{q}^{-}$formation with $q=5-14$ (Fig. 40), measured by the Kaiserslautern group with the LPA method at energy widths around $2 \mathrm{meV}$ (Barsotti et al., 2002a). In view of the much improved resolution of the LPA experiment, at least the peak structure, located near $80 \mathrm{meV}$ in the Innsbruck data, should be present in the LPA data. Reasons for the different observations may possibly be found in the scenario, by which $\left(\mathrm{O}_{2}\right)_{q}^{-}$anions $(q=1-3)$ are formed. According to Matejcik et al. (1999) the attachment process proceeds in such a way that the incoming electron is primarily trapped at one oxygen molecule in neutral clusters with sizes dominated by the range $N=10-20$. Subsequent substantial evaporation then yields the observed 


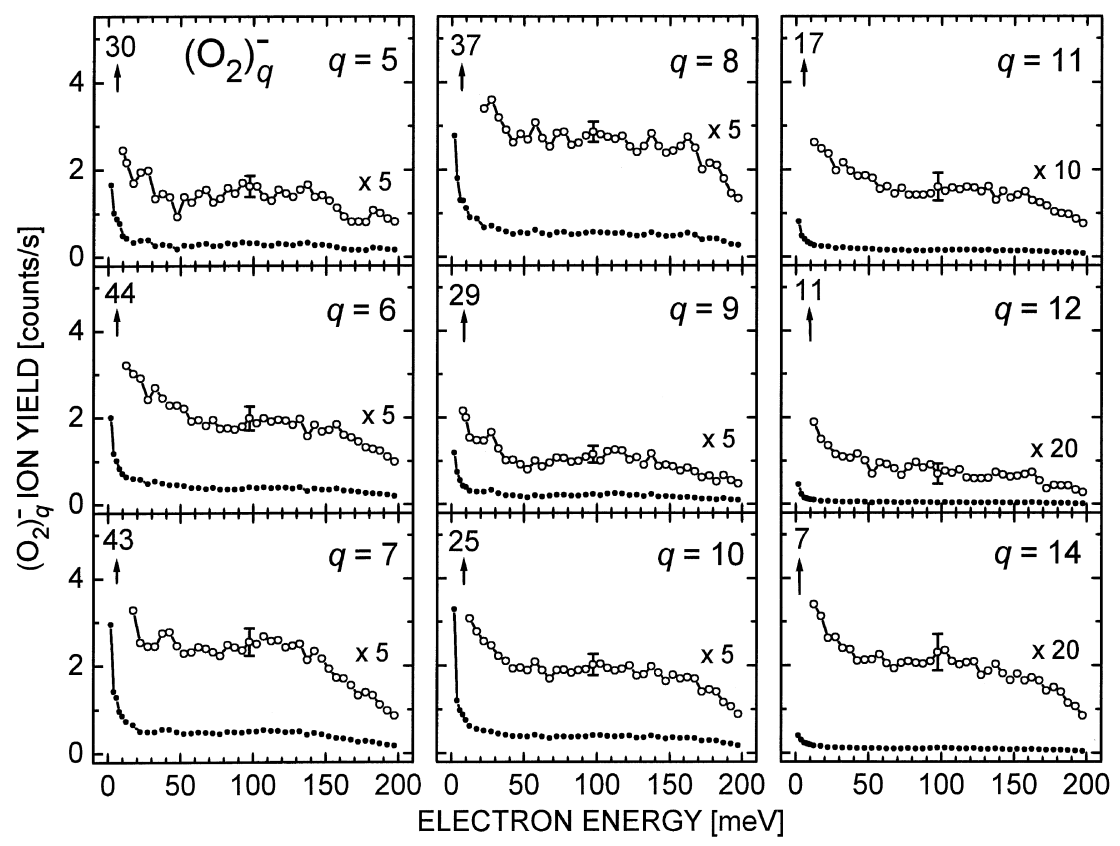

FIG. 40. Low-energy electron attachment spectra for formation of oxygen cluster anions $\left(\mathrm{O}_{2}\right)_{q}^{-}$, measured with about $2 \mathrm{meV}$ resolution. The numbers above the arrows near-zero energy give the cluster anion yields for RET at $n \approx 260$ (from Barsotti et al., 2002a).

anions according to the following reaction scheme:

$$
\mathrm{e}^{-}(E)+\left(\mathrm{O}_{2}\right)_{N} \rightarrow\left(\mathrm{O}_{2}\right)_{N}^{-*} \rightarrow\left(\mathrm{O}_{2}\right)_{q}^{-}+(N-q) \mathrm{O}_{2}
$$

For this reaction to be exothermic at zero electron energy, the total binding energy of the $\left(\mathrm{O}_{2}\right)_{q}^{-}$ion (composed of the adiabatic electron affinity of $\mathrm{O}_{2}$ plus the bond energies of the additional $(q-1) \mathrm{O}_{2}$ molecules) has to exceed the total binding energy $E_{N}$ of the neutral precursor cluster of size $N$. In particular, $\mathrm{O}_{2}^{-}$formation requires that $E_{N}$ be smaller than $E A_{\text {ad }}\left(\mathrm{O}_{2}\right)=$ 0.451 (7) eV. According to calculations by Matejcik et al. (1999), scaled to reproduce the cohesive energy of bulk oxygen, the total binding energy of neutral $\left(\mathrm{O}_{2}\right)_{N}$ clusters becomes larger than $E A_{\mathrm{ad}}\left(\mathrm{O}_{2}\right)$ for $N \geq 14$. Correspondingly, process (43) for $q=1$ is energetically not possible when zero energy electrons attach to very cold clusters with $N \geq 14$, but may proceed when the electrons possess a sufficient amount of kinetic energy $E$ (for $N=20$, the threshold energy for $\mathrm{O}_{2}^{-}$formation amounts to about 
$E=0.3 \mathrm{eV})$. In contrast, formation of $\left(\mathrm{O}_{2}\right)_{q}^{-}$anions with $q \geq 2$ is exothermic for all neutral sizes up to about $N=25$ even at zero electron energy. If a size range of neutral clusters is active as precursor in the cluster anion formation of a particular anion size $q$ according to (43), the observation of vibrational structure implies a sufficiently small inhomogeneous broadening of the vibrational resonance. Model calculations of the distribution function of the adiabatic electron affinity of clusters with sizes $N=10,15$ and 20 (Fig. 8 in Matejcik et al., 1999) for the various cluster structures and positions of the solvated $\mathrm{O}_{2}^{-}$in the cluster showed the following behavior at low cluster temperatures $T_{\mathrm{C}}$ : for sufficiently cold (i.e. solid) clusters $\left(T_{\mathrm{C}} \leq 20 \mathrm{~K}\right)$, the width (FWHM) of the distribution function for a particular $N$ amounted to about $0.07 \mathrm{eV}$. Moreover, the peak position of the distribution remained nearly the same (close to $0.8 \mathrm{eV}$ ) for $N=15$ and 20 while it shifted to $0.7 \mathrm{eV}$ for $N=10$. For (partially) molten clusters, as shown for $T_{\mathrm{C}}=40 \mathrm{~K}$ (Matejcik et al., 1999), the distribution was found to become wider by about a factor of 2 and thus too broad as to resolve vibrational structure even for a single precursor size. These calculations thus suggest that the absence of vibrational structure in our attachment spectra may be due to the fact that the oxygen clusters in our experiment were not sufficiently cold. From simulations of the peak structure, assuming solid oxygen clusters with a certain size range, Matejcik et al. concluded that neutral clusters around $N=15$ (ranging from 13 to 20) are responsible for their attachment spectra with $q=1,2,3$. This size selectivity was not explained. The authors also did not comment why the same range should be responsible for the formation of $\mathrm{O}_{2}^{-}, \mathrm{O}_{4}^{-}$, and $\mathrm{O}_{6}^{-}$ions (note that the peak structures for cluster anions with $q=1,2$, and 3 was found to be located within their uncertainties - at the same energies). For $\mathrm{O}_{2}^{-}$formation, one would actually expect (in view of the energetic restriction that $N$ may not be larger than 13) that the average size of the neutral clusters involved in anion formation is significantly smaller than for dimer and trimer anion formation with the result that the peaks in the $\mathrm{O}_{2}^{-}$attachment spectrum appear at higher energies than those in the dimer and trimer anion spectra.

In this connection it is appropriate to recall that the Berlin group found a clear difference in the peak location of the yield for $\left(\mathrm{O}_{2}\right)_{q}^{-}$formation between $q=1(0.7 \mathrm{eV})$ and $q \geq 2(0.4 \mathrm{eV})$ (Hashemi et al., 1992). This difference is, on the one hand, compatible with a scenario that, on average, $\mathrm{O}_{2}^{-}$formation involves neutral clusters of smaller size than those responsible for $\left(\mathrm{O}_{2}\right)_{q}^{-}(q \geq 2)$ formation. In the light of the Innsbruck observations (yielding the maximum yield of small oxygen cluster anions at $0 \mathrm{eV}$ ) the Berlin results may be possibly understood if - as proposed by Matejcik et al. (1999) - the neutral precursor clusters had a substantially smaller size in the 
Berlin experiment. It is desirable for a better understanding of these different observations to carry out additional high resolution experiments which allow to vary the neutral cluster size distribution and the cluster temperature.

\section{C.2. Nitrous Oxide Clusters}

Threshold electron attachment to $\mathrm{N}_{2} \mathrm{O}$ clusters via Rydberg electron transfer (RET) close to zero energy (Rydberg binding energy $0.2 \mathrm{meV}$ ) produces heterogeneous $\left(\mathrm{N}_{2} \mathrm{O}\right)_{q} \mathrm{O}^{-}$and homogeneous $\left(\mathrm{N}_{2} \mathrm{O}\right)_{p}^{-}$cluster anions in a highly size selective way (Kraft et al., 1990). The dominant anion species were observed to be $\left(\mathrm{N}_{2} \mathrm{O}\right)_{q} \mathrm{O}^{-}$with $q=5,6$ and $\left(\mathrm{N}_{2} \mathrm{O}\right)_{p}^{-}$with $p=7,8$, the latter being generally less intense than the former. These cluster anion intensities suggest resonant capture of zero energy electrons for neutral cluster size $N \approx 8$ with subsequent stabilization by intermolecular vibrational redistribution, by evaporation $\left(\mathrm{N}_{2} \mathrm{O}\right.$ emission) or by dissociation (release of $\mathrm{N}_{2}$ fragments).

In Fig. 41 we present the energy dependent yield for formation of heterogeneous $\left(\mathrm{N}_{2} \mathrm{O}\right)_{q} \mathrm{O}^{-}$ions $(q=4-9,11,13)$ and in Fig. 42 that of homogeneous $\left(\mathrm{N}_{2} \mathrm{O}\right)_{p}{ }^{-}$ions $(p=8,9,11,13)$, as observed over the energy range 1.5$178 \mathrm{meV}$ by Leber et al. (2000c). For all sizes $q$ and $p$ a sharp increase towards $0 \mathrm{eV}$ is observed below $E \approx 15 \mathrm{meV}$, indicative of an s-wave attachment process. In all these spectra, astoundingly narrow peaks are observed at energies $E_{\mathrm{R}}$ close to, but not identical with the excitation energies $E\left(v_{\mathrm{i}}\right)$ for the bending $\left(v_{2}=1,2\right)$ and the $\mathrm{N}-\mathrm{O}$ stretching $\left(v_{1}=1\right)$ vibrational mode of free $\mathrm{N}_{2} \mathrm{O}$ molecules. The widths (FWHM) of these peaks (around $2.5 \mathrm{meV}$ for the bending fundamental, around $4-5 \mathrm{meV}$ for the bending overtone and the $\mathrm{N}-\mathrm{O}$ stretching mode (i.e. substantially broader than the experimental resolution of $1.2 \mathrm{meV}$ ) are essentially independent of cluster ion size. They are the narrowest resonances detected so far in electron scattering for any cluster or polyatomic molecule. They have been interpreted as vibrational Feshbach resonances (VFRs) (Weber et al., 1999a, Leber et al., 2000c), i.e. temporary anion states of the type $\left[\left(\mathrm{N}_{2} \mathrm{O}\right)_{N-1}\left(\mathrm{~N}_{2} \mathrm{O}\left(v_{\mathrm{i}}>0\right)\right]^{-}\right.$. The energy location and the long lifetime of these resonances (on the order of $2 \times 10^{-13} \mathrm{~s}$ for the $v_{2}=1$ VFRs, if inhomogeneous broadening due to contributions from different neutral precursors is neglected) are compatible with the idea of a weakly bound diffuse excess electron attached to an essentially unperturbed neutral $\left(\mathrm{N}_{2} \mathrm{O}\right)_{N-1} \mathrm{~N}_{2} \mathrm{O}\left(v_{\mathrm{i}}>0\right)$ cluster by long range forces. The widths of the resonances increase with electron energy, probably due to rising phase space for the most probable decay mechanism of the resonances, namely autodetachment of the captured electron. The peak positions exhibit small 

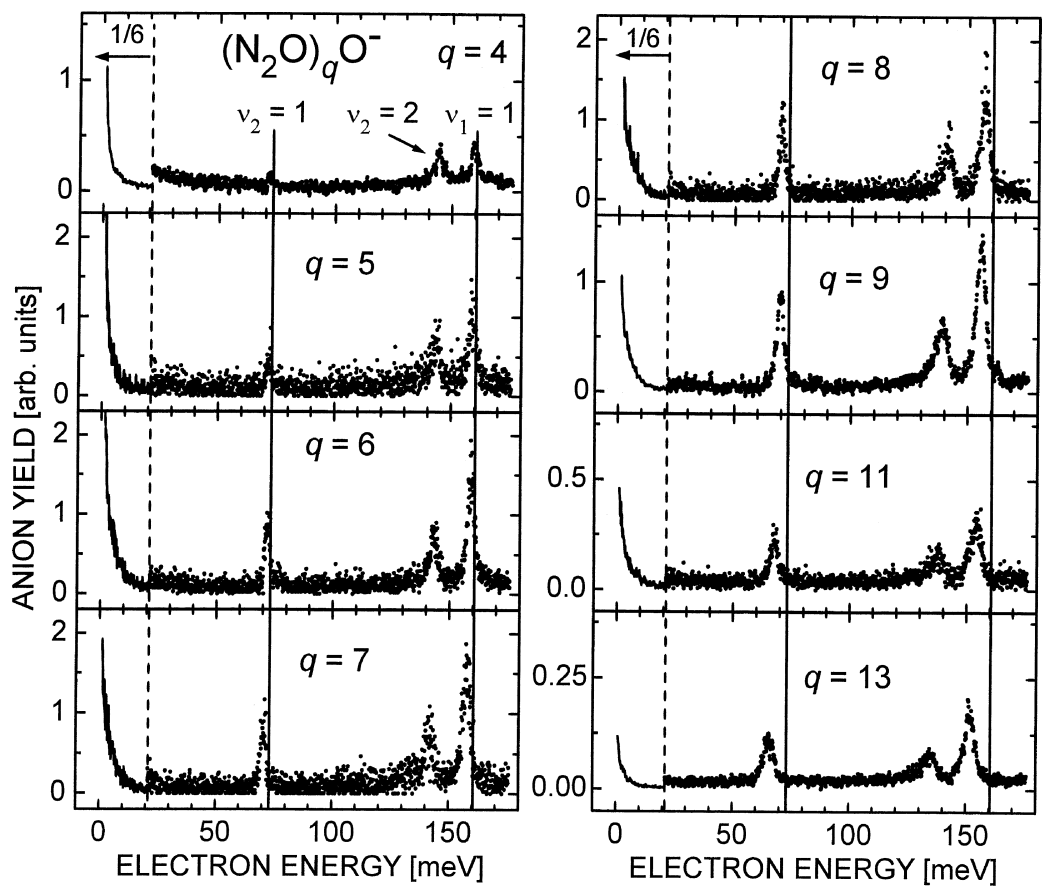

FIG. 41. Low-energy electron attachment spectra for formation of heterogeneous $\left(\mathrm{N}_{2} \mathrm{O}\right)_{\mathrm{q}} \mathrm{O}^{-}$ cluster anions from $\left(\mathrm{N}_{2} \mathrm{O}\right)_{N}$ clusters $(q<N)$. The full vertical lines denote the energy positions of the $v_{2}=1$ and the $v_{1}=1$ intramolecular vibrational excitation in $\mathrm{N}_{2} \mathrm{O}$ (from Leber et al., 2000c).

redshifts, defined as the quantity $\Delta \equiv E\left(v_{\mathrm{i}} ; N\right)_{\mathrm{C}}-E_{\mathrm{R}}$, which increase with cluster ion size; here $E\left(\nu_{i} ; N\right)_{C}$ denotes the intramolecular excitation energy of the $v_{\mathrm{i}}$ mode in the neutral cluster with size $N$ which acts as the precursor for the observed anion of size $q$ or $p$. It has to be emphasized that attachment processes involving slow secondary electrons from inelastic collisions with $\mathrm{N}_{2} \mathrm{O}$ monomers are ruled out for reasons of low target density and the inability to produce the observed cluster size dependent redshift.

The VFRs evolve to the observed homogeneous and heterogeneous cluster anions in different ways. Energy redistribution into soft intermolecular modes may lead to long-lived homogeneous ions with size $p=N$. Homogeneous cluster anions with $p<N$ are produced upon evaporation of at least one $\mathrm{N}_{2} \mathrm{O}$ molecule. Formation of heterogeneous cluster anions involves removal of one $\mathrm{N}_{2}$ fragment, possibly accompanied by release of 

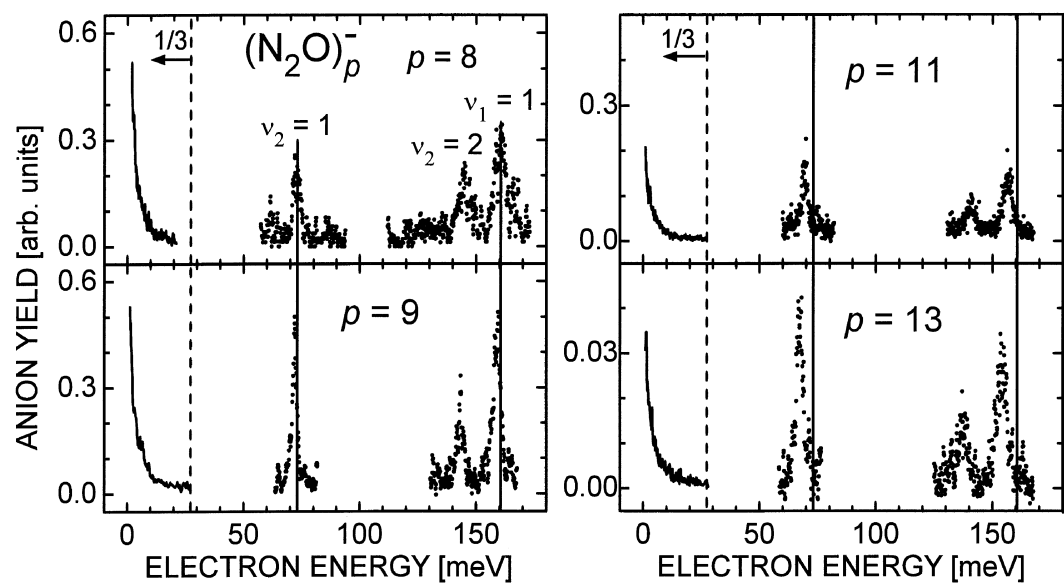

FIG. 42. Low-energy electron attachment spectra for formation of homogeneous $\left(\mathrm{N}_{2} \mathrm{O}\right)_{p}^{-}$ cluster anions from $\left(\mathrm{N}_{2} \mathrm{O}\right)_{N}$ clusters $(p \leq N)$. The full vertical lines denote the energy positions of the $v_{2}=1$ and the $v_{1}=1$ intramolecular vibrational excitation in $\mathrm{N}_{2} \mathrm{O}$ (from Leber et al., 2000c).

$\mathrm{N}_{2} \mathrm{O}$ units. The anion intensities in the threshold mass spectrum and correlations in the redshifts of the heterogeneous anions $\Delta_{\text {het }}(q)$ and of the homogeneous anions $\Delta_{\text {hom }}(p)$ suggest that neutral precursor clusters with size $N$ on average are correlated with homogeneous anions of size $p(\leq N)$ and heterogeneous cluster anions $\left(\mathrm{N}_{2} \mathrm{O}\right)_{q} \mathrm{O}^{-}$with size $q=p-(2$ or 3$)$ (Leber et al., 2000c).

In view of the existence of the vibrational Feshbach resonances $\left[\left(\mathrm{N}_{2} \mathrm{O}\right)_{\mathrm{N}-1}\left(\mathrm{~N}_{2} \mathrm{O}\left(v_{\mathrm{i}}>0\right)\right]^{-}\right.$, it is natural to infer the presence of truly bound, nonautodetaching anion states $\left[\left(\mathrm{N}_{2} \mathrm{O}\right)_{N-1}\left(\mathrm{~N}_{2} \mathrm{O}\left(v_{\mathrm{i}}=0\right)\right]^{-}\right.$without intramolecular vibrational excitation (see the model calculations below). Such bound states are not accessible in free electron collisions, but can be formed by Rydberg electron transfer (RET) as shown, e.g., by Desfrançois et al. (1996) for dipole bound anions. The strong rise in the free electron attachment yield towards zero energy is attributed to the influence of these weakly bound $\left(\mathrm{N}_{2} \mathrm{O}\right)_{N}^{-}$capture states without intramolecular excitation (but possibly with some intermolecular excitation). The rather sharp drop in the RET-induced anion intensities, as observed at threshold $(n \cong 250)$ for $q>6$ and $p>9$, appears to be mainly due to the increasing binding energies of the $\left[\left(\mathrm{N}_{2} \mathrm{O}\right)_{N-1}\left(\mathrm{~N}_{2} \mathrm{O}\left(v_{\mathrm{i}}=0\right)\right]^{-}\right.$states with rising $N$ which correlate with a shift of the maximum RET rate coefficient towards lower principal quantum numbers $n$ (Desfrançois et al., 1996), as in fact observed experimentally (Kraft et al., 1990). 
In the discussion above, it has been assumed for simplicity that a particular observed VFR is associated with a single size $N$ only. This assumption, however, is uncertain in view of the fact that the resonance widths, especially for the two higher lying VFRs, are larger than or at least similar to the redshift. Even if the observed VFRs correspond to a mixture of neighbouring sizes $N$ with a mean value $\langle N\rangle$ and if $N$ is not identical to $p$, it is still true, however, that the relation $q=p$-( 2 or 3 ) holds.

In order to provide a qualitative understanding of the small VFR redshifts, Leber et al. (2000c) performed simple model calculations for the binding energy $E_{\mathrm{B}}=-\Delta$ of the captured electron in the VFR state $\left[\left(\mathrm{N}_{2} \mathrm{O}\right)_{N-1} \mathrm{~N}_{2} \mathrm{O}\left(v_{\mathrm{i}}>0\right)\right]^{-}$relative to the energy $E_{N}$ of the neutral $\left[\left(\mathrm{N}_{2} \mathrm{O}\right)_{N-1} \mathrm{~N}_{2} \mathrm{O}\left(v_{\mathrm{i}}>0\right)\right]$ cluster which carries the same amount of intramolecular vibrational energy $\left(E_{\mathrm{R}}=E_{N}+E_{\mathrm{B}}\right)$. The authors ignored geometrical arrangement effects of the cluster constituents as well as the intramolecular vibration and assumed that the binding energy $E_{\mathrm{B}}$ is simply due to the combined effects of the long-range attraction between the electron and the cluster and the short range interaction $U_{0}$ at distances smaller than the cluster radius $R_{N}$. Leber et al. (2000c) took into account the polarization attraction $V_{\text {pol }}=-N \mathrm{e}^{2} \alpha\left(\mathrm{N}_{2} \mathrm{O}\right) /\left(2 \mathrm{r}^{4}\right)$ (neglecting, in the average over the cluster, the effects associated with the weak dipole moment and the quadrupole moment of the $\mathrm{N}_{2} \mathrm{O}$ molecules) and cut it off at the cluster radius; at electron-cluster distances smaller than $R_{N}=R_{0}(1.5 N)^{1 / 3}\left(R_{0}=\right.$ effective radius of a monomer) a constant potential energy $U_{0}$ was used and treated as a parameter. In Fig. 43 we sketch the potential model for $U_{0}=+0.2 \mathrm{eV}$ and $N=11$ and the probability density for the wave function of the corresponding weakly bound electron (Leber et al., 2000c). As expected in view of the weak binding energies, the radial extension of the electron in these resonance states is quite large as illustrated in Fig. 43 for the VFR state with size $N=11$.

Figure 44 shows the results of these calculations for the electron binding energies to clusters with $N=4-15$, using several constant, repulsive values for the short range potential $U_{0}$, the isotropic monomer polarizability $\alpha\left(\mathrm{N}_{2} \mathrm{O}\right)=20.4 \mathrm{a}_{0}^{3}[25]$ and $R_{0}=3 \mathrm{a}_{0}\left(\mathrm{a}_{0}=\right.$ Bohr radius $)$. For comparison with the calculated binding energies (curves), the experimentally derived binding energies $E_{\mathrm{B}, \text { het }}(N, q)=-\Delta_{\text {het }}(q+2)$ (full symbols) and $E_{\mathrm{B}, \mathrm{hom}}(N, p)=$ $-\Delta_{\text {hom }}(p)$ (open symbols) (i.e. we assume $N=p=q+2$ ) are included in Fig. 44. For the intramolecular excitation energies $E\left(v_{\mathrm{i}} ; N\right)_{\mathrm{C}}$ in $\mathrm{N}_{2} \mathrm{O}$ clusters the values, reported by Gauthier (1988) for $v_{2}=2$ and $v_{1}=1$, and for $v_{2}=1$ the monomer value were adopted. One observes reasonable agreement between the thus calculated and the observed resonance positions for $U_{0}=+0.2 \mathrm{eV}$. The use of more elaborate potentials $U_{0}$ (Stampfli, 1995) does not provide deeper insight at the present level of accuracy. 


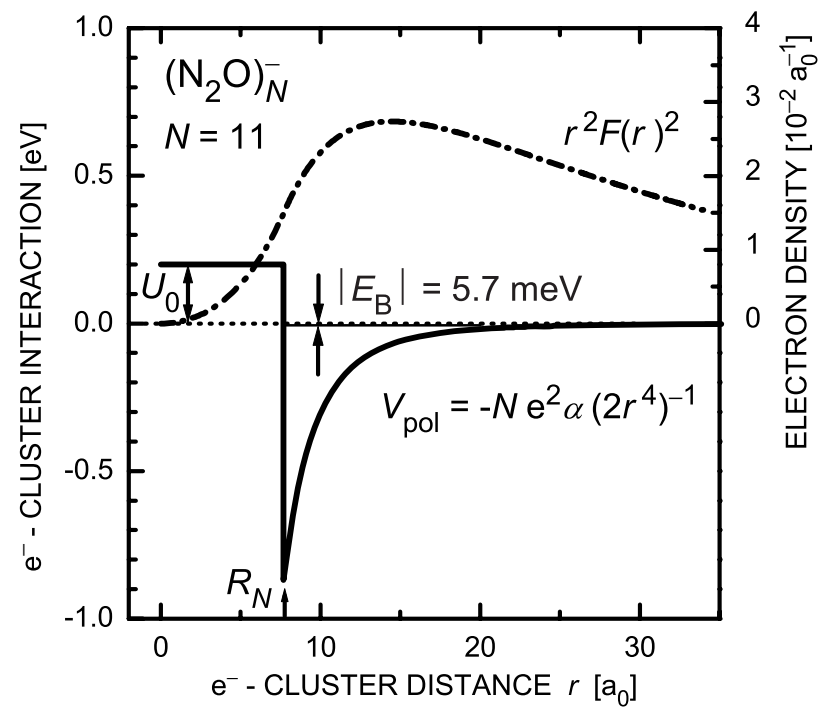

FIG. 43. Probability density (chain curve) for the radial wave function of the electron, weakly bound to the $\left(\mathrm{N}_{2} \mathrm{O}\right)_{11}$ cluster and the model potential (full curve) used to calculate this wave function (Leber et al., 2000c).

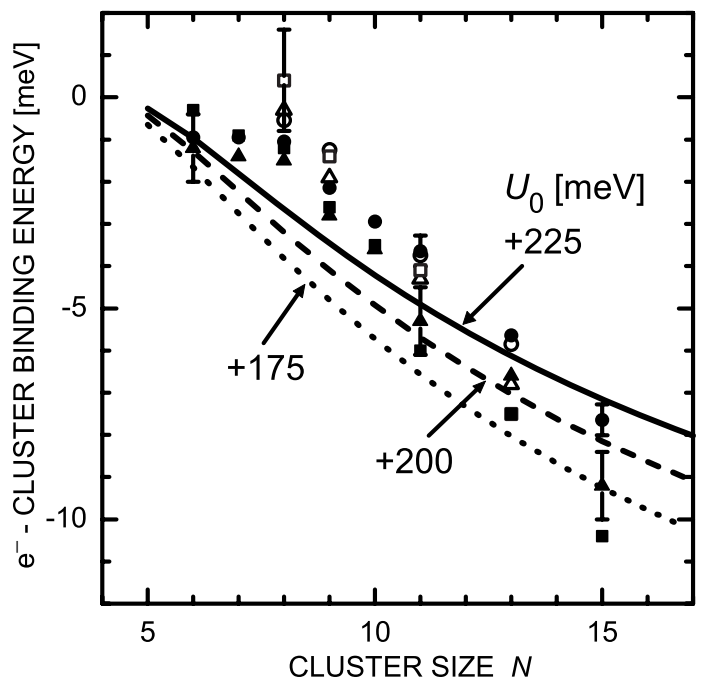

FIG. 44. Comparison of calculated and experimentally determined binding energies for weakly bound $\left(\mathrm{N}_{2} \mathrm{O}\right)_{N}^{-}$anions, for details see text (Leber et al., 2000c). 


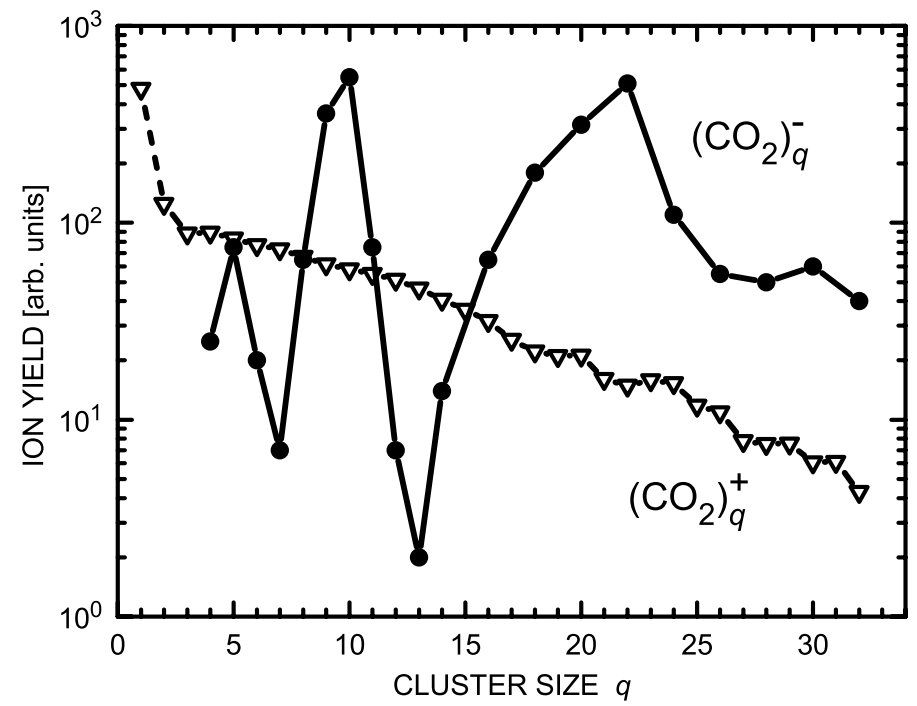

FIG. 45. Cluster ion mass spectra for $\mathrm{CO}_{2}$ clusters (after Barsotti et al., 2002a). Open triangles: mass spectrum of positive ions resulting from $85 \mathrm{eV}$ electron impact ionization; full circles: mass spectrum of anions due to threshold electron attachment involving $\mathrm{K}^{* * *}(n d$, $n \approx 260$ ) Rydberg atoms.

\section{C.3. Carbon Dioxide Clusters}

Some properties of the $\mathrm{CO}_{2}$ molecule have been discused in Section IV.B5. According to molecular dynamics simulations (Etters et al., 1981; Tsukada et al., 1987; Torchet et al., 1996; Maillet et al., 1998), neutral carbon dioxide clusters possess icosahedral structure (at least for sizes up to about $N=20$ ) with predicted binding energies around $0.14 \mathrm{eV}$ per molecule for $N=13$ (Maillet et al., 1998); at sizes above about $N=30$, a transition to bulk cubic structure has been predicted, and this bulk structure has been experimentally verified for $N$ above about 100 (Torchet et al., 1996). Some information on cluster anion structures is also available. In agreement with photoelectron spectroscopic observations (Tsukuda et al., 1997a), $a b$ initio calculations of the equilibrium structures and stabilities for different isomers of small $\left(\mathrm{CO}_{2}\right)_{q}^{-}$cluster anions $(q \leq 6)$ find (Saeki et al., 2001) that the dimer $\mathrm{C}_{2} \mathrm{O}_{4}^{-}$acts as the core in clusters with $q=2-5$. They predict evaporation energies for dissociation of one $\mathrm{CO}_{2}$ unit from various isomers of $\left(\mathrm{CO}_{2}\right)_{q}^{-}$anions in the range $0.1-0.4 \mathrm{eV}$, rising substantially (by about $0.15 \mathrm{eV}$ ) when going from $q=5$ to $q=4$. Tsukada et al. (1987) developed a theory for the attachment of slow electrons to van der Waals clusters and applied it to $\mathrm{CO}_{2}$ clusters. They predicted the existence of 
a threshold size for attachment and a sharp decrease of the attachment cross-section with rising electron energy.

Previous experimental work of cluster anion formation in energycontrolled electron collisions with carbon dioxide clusters includes low-resolution beam studies over extended energy ranges (Compton, 1980, Stamatovic et al., 1985a, b; Knapp et al., 1985, 1986a), investigations by Rydberg electron transfer (RET) (Kondow, 1987; Kraft et al., 1989, 1991; Misaizu et al., 1991) and the recent LPA studies (energy width about $1 \mathrm{meV}$ ) (Leber et al., 2000b; Barsotti et al., 2002a). Anion mass spectra obtained by RET revealed an intriguing anion cluster size dependence. In Fig. 45, the relative intensities for $\left(\mathrm{CO}_{2}\right)_{q}^{+}$cluster ions due to $85 \mathrm{eV}$ electron impact (open triangles) are compared with the relative intensities of the $\left(\mathrm{CO}_{2}\right)_{q}^{-}$ cluster anions (full circles), obtained in RET with a narrow $n$ band of $\mathrm{K}^{* * *}(n \mathrm{~d})$ Rydberg atoms, centered around $n=260$ (electron binding energy $0.2 \mathrm{meV}$ ). Note that, in contrast to the anion mass spectra shown in Fig. 2 of Barsotti et al. (2002a), the anion yield in Fig. 45 was obtained with the ion optics optimized for each cluster size. $\left(\mathrm{CO}_{2}\right)_{q}{ }^{+}$ions $(q \geq 1)$ dominate the positive ion mass spectrum and exhibit a nearly exponential intensity decrease with rising cluster size $q$. As expected on energetic grounds, only homogeneous ions $\left(\mathrm{CO}_{2}\right)_{q}^{-}(q>3)$ are observed in the anion mass spectra due to electron attachment to carbon dioxide clusters, both in Rydberg electron transfer (RET) involving $\mathrm{K}^{* *}(n d)$ atoms $(n=14-260)$ and in free electron attachment $(E=1-200 \mathrm{meV})$. Compared with the positive ion spectrum, the RET-induced anion cluster mass spectrum is completely different: the threshold anion spectrum exhibits local maxima at $q=5$ and 10 as well as a broad maximum around $q=22$, and deep, local minima at $q=7$ and 13. Anion cluster mass spectra at lower principal quantum numbers $(n \leq 30)$ (Kondow, 1987; Kraft et al., 1989; Leber, 2000b) differ significantly from the threshold spectrum in Fig. 45 and indicate a substantial dependence on electron energy over a narrow range, as will be understood through the observations in the free electron attachment spectra.

In Fig. 46 we present the energy dependent yield for $\left(\mathrm{CO}_{2}\right)_{q}^{-}$cluster anions for selected sizes $q$ from the covered range $q=4-32$ over the energy range from $1 \mathrm{meV}$ up to $200 \mathrm{meV}$ (Barsotti et al., 2000a). For small cluster sizes, two clearly separated series of resonances are observed which exhibit redshifts increasing by about $12 \mathrm{meV}$ per monomer unit. The key spectrum observed for $q=5$ (in which the peaks exhibit the narrowest widths) shows a distinct zero energy peak (indicative of s-wave attachment), a rather sharp resonance peaking at about $52 \mathrm{meV}$ and a double-peak structure with a center-of-gravity around $134 \mathrm{meV}$. These three peaks are attributed to vibrationally excited temporary cluster anion states (vibrational Feshbach 

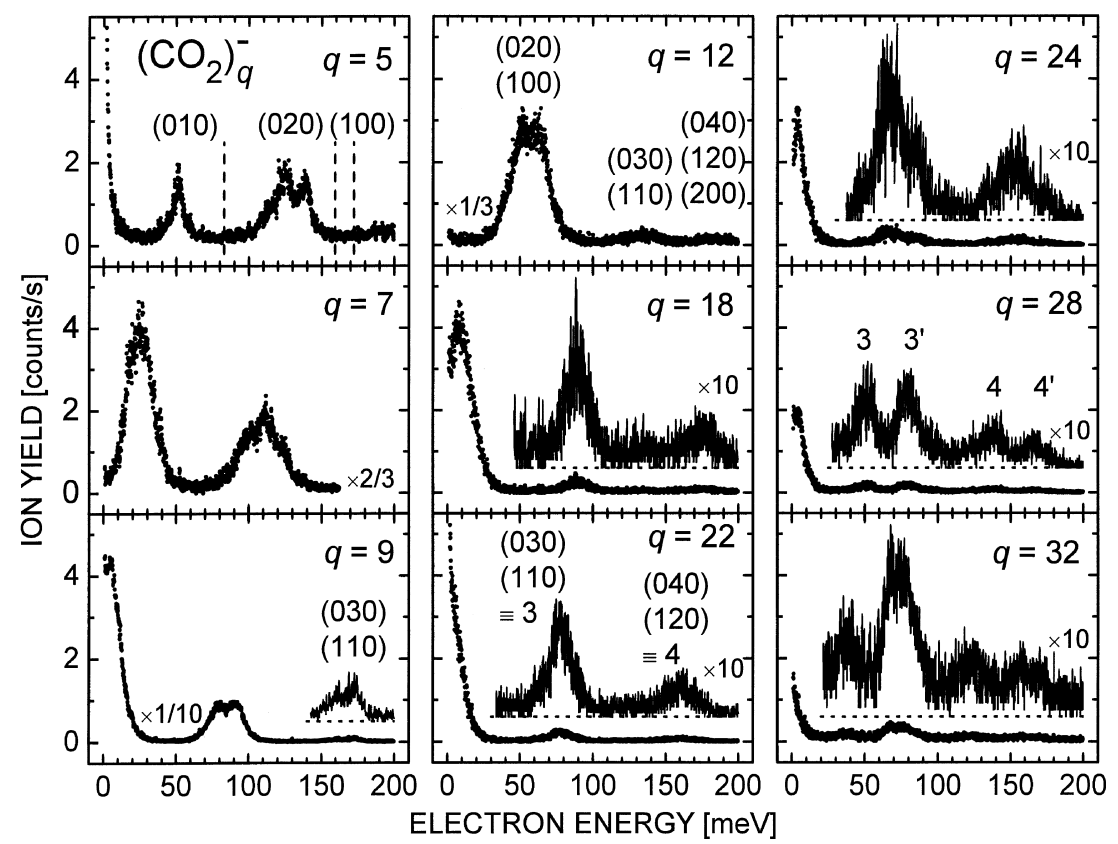

FIG. 46. Low-energy electron attachment spectra for formation of $\left(\mathrm{CO}_{2}\right)_{q}^{-}$cluster anions $(q=4-32)$ from $\left(\mathrm{CO}_{2}\right)_{N}$ clusters $(q \leq N)$. For $q=5$, the energy positions of the $\left(v_{1} v_{2} v_{3}\right)=(010)$ and the Fermi-coupled (020)/(100) vibrational modes of the $\mathrm{CO}_{2}$ monomer are indicated by vertical dashed lines. The incremental redshift of the vibrational Feshbach resonances amounts to about $12 \mathrm{meV}$ per monomer unit. For $q>7$, the $3 \equiv(030) /(110)$ and, for $q>10$, the $4 \equiv$ $(040) /(120) /(200)$ vibrational modes are shifted into the electron energy range covered by the LPA experiment. For $q>24$, a doubling of the Feshbach resonance peak structure is observed $\left(3 / 3^{\prime}\right.$ and $\left.4 / 4^{\prime}\right)$ (after Barsotti et al., 2002a).

resonances VFRs) of the type $\left[\left(\mathrm{CO}_{2}\right)_{N-1} \mathrm{CO}_{2}\left(v_{1} v_{2} v_{3}\right)\right]^{-}$with $\left(v_{1} v_{2} v_{3}\right)=(010)$ and $(020) /(100)$, respectively, which evolve into the observed long-lived $\left(\mathrm{CO}_{2}\right)_{q}^{-}=5$ anions either by redistribution of the vibrational energy among soft modes of the cluster with formation of a metastable cluster ion with $q=N$ or by evaporation of a small number of $\mathrm{CO}_{2}$ units (most likely $N-q=1$, see below). As expected for VFRs, their energies are redshifted from those of the neutral $\left[\left(\mathrm{CO}_{2}\right)_{N-1} \mathrm{CO}_{2}\left(v_{1} v_{2} v_{3}\right)\right]$ precursor. In contrast to the sharp VFRs observed for $\mathrm{N}_{2} \mathrm{O}$ clusters which exhibit small redshifts in the meV range (see above), the redshift is substantially larger for $\mathrm{CO}_{2}$ clusters, indicating a stronger interaction of the resonantly-captured electron with $\mathrm{CO}_{2}$ clusters than with $\mathrm{N}_{2} \mathrm{O}$ clusters. In line with this observation the resonance widths are larger for $\mathrm{CO}_{2}$ clusters than for $\mathrm{N}_{2} \mathrm{O}$ clusters. For any particular $\left(\mathrm{CO}_{2}\right)_{q}^{-}$cluster ion, the width of the observed resonances 


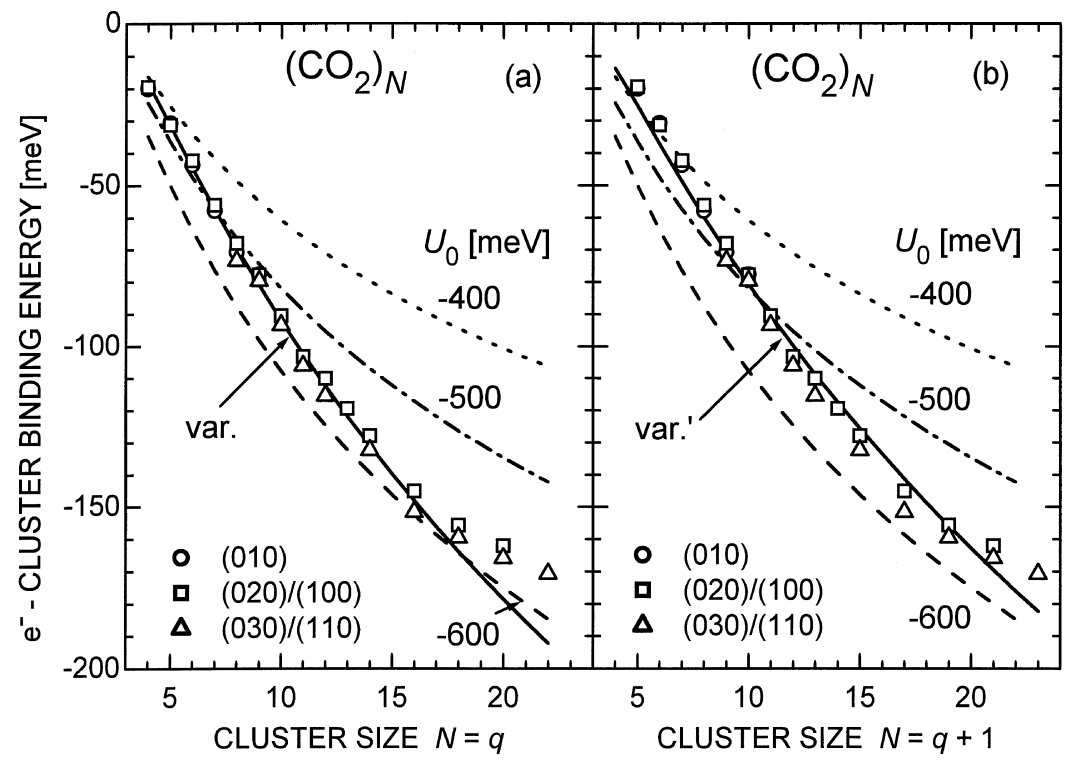

FIG. 47. Comparison of calculated and measured binding energies for VFRs in $\mathrm{CO}_{2}$ clusters, assuming $N=q$ (a) and $N=q+1$ (b), for details see text (after Barsotti et al., 2002a).

contains contributions due to the intrinsic resonance widths and resonance positions of all involved neutral precursors which participate in VFR formation, i.e. the observed resonances are in general inhomogeneously broadened. In view of the substantial redshift of about $12 \mathrm{meV}$ per added $\mathrm{CO}_{2}$ unit and the comparatively narrow intrinsic width of the VFRs, the width in conjunction with the redshift of the VFR allows rather direct conclusions to be drawn on the size range of the involved neutral precursors. For $q=5$ the resonances exhibit the smallest widths, and it is plausible that the attachment spectrum is predominantly associated with a single neutral precursor size (with the possibility of inhomogeneous broadening due to contributions from different conformations for that cluster size). At this narrow peak width, the Fermi-coupled pair (020)/(100) is quite well resolved. Interestingly, we find the two peaks at similar intensities in contrast to the situation for vibrationally inelastic scattering of low energy electrons from $\mathrm{CO}_{2}$ molecules where, close to the threshold for the pair $(020) /(100)$, the higher energy component is excited almost exclusively (Allan, 2001a). This finding has been associated with the influence of a virtual state which has been theoretically predicted for low-energy electron scattering from carbon dioxide (Morrison, 1982; 
Estrada and Domcke, 1985; Morgan, 1998) and recently also been confirmed by total scattering studies at very low energies (Field et al., 2001b).

For $q=4$ and $q \geq 6$, the (010) resonances appear to be significantly broader than for $q=5$; this may indicate contributions from two neighbouring neutral precursor sizes, but could also be caused (at least in part) by the influence of different conformations for a particular $N$. For larger sizes $(q>7)$, additional series of peaks, attributed to resonances associated with the Fermi-coupled pair $(030) /(110)$ and the triplet $(040) /(120) /(200)$, are successively shifted into the studied energy range. The evolution of these series is clearly seen in the attachment spectra for the clusters anions with sizes $q=14-32$. The trends are similar to those observed at smaller anion size, but the redshifts per added molecular unit decrease somewhat at higher $q$. For cluster sizes $q$ above 24 an interesting doubling of the VFR peak structure is observed in both the $(030) /(110) \equiv 3$ and $(040) /(120) /(200) \equiv 4$ series; the 3 and 4 series evolve 'normally' towards lower energies with intensities decreasing towards higher $q$ while the 'new' series $3^{\prime}$ ' and 4 ' are observed at $30-40 \mathrm{meV}$ higher energies relative to the 3 and 4 series, respectively, with intensities rising towards larger $N$. We tentatively attribute these two series to arise from the coexisting icosahedral (series 3,4) and bulk cubic (series $3^{\prime}, 4^{\prime}$ ) cluster structures, with the former losing and the latter gaining importance towards higher $N$. According to molecular dynamics simulations for carbon dioxide clusters, the icosahedral (low $N$ ) to bulk cubic (high $N$ ) transition is predicted to occur for sizes around $N=30$ (Torchet et al., 1996).

The attachment spectra shown in Fig. 46 offer the following explanation for the strongly $q$-dependent ion intensities in the mass spectrum resulting from threshold electron attachment (Fig. 45) with minima at $q=7$ and 13, a clear maximum at $q=10$ and another broad maximum for $q$ around 21 . Enhanced cluster ion intensities are found for $q$-values for which a substantial overlap of a VFR with zero electron energy exists. For $q=9$, 10 , the (010) resonance has moved close to zero energy, for $q=16-22$, the $(020) /(100)$ resonance pair has a more or less substantial overlap with zero energy. The intensity rise in the threshold attachment mass spectrum from $q=6$ to $q=5$ may be attributed to an influence of $\left(\mathrm{CO}_{2}\right)_{N}^{-}$capture states without intramolecular excitation (but possibly some intermolecular excitation). One would expect such an influence to be even stronger for $\left(\mathrm{CO}_{2}\right)_{4}^{-}$formation, but the autodetachment rates of the temporary $\left(\mathrm{CO}_{2}\right)_{N}^{*}$ negative ions are expected to rise towards low $N$ and thus their survival probability towards stabilization and detection as long-lived anions should decrease. For further discussions including variations of the cluster anion mass spectrum with increasing electron energy see Barsotti et al. (2002a). 
Application of the simple spherical model for the electron binding energies $E_{\mathrm{B}}$, sketched in Section C.2, reproduces the redshifts when - in contrast to the situation for $\mathrm{N}_{2} \mathrm{O}$ clusters - attractive short range potentials are used (Leber et al., 2000b; Barsotti et al., 2002a). Figure 47 shows calculated electron binding energies to clusters with $N=4-22$ (dashed curves), as obtained with realistic choices of the short range potential $U_{0}$ (for further results see Leber et al. (2000b)). For comparison, the experimentally derived binding energies $E_{\mathrm{B} \text {,exp }}$ for the (010), (020)/(100), (030)/(110) and $(040) /(120) /(200)$ resonance series, as calculated from the center-of-gravity resonance positions $E_{\mathrm{R}}$ through $E_{\mathrm{B}, \exp }=E_{\mathrm{R}}-E_{q}$, are included, using two different assumptions: (a) $N=q$ and (b) $N=q+1$. In view of the fact that the intramolecular excitation energies $E_{N}$ in $\mathrm{CO}_{2}$ clusters deviate from those in the $\mathrm{CO}_{2}$ monomer $E_{1}$ (see IV.B.5.a) by no more than about $\pm 1 \mathrm{meV}$ (see references in Leber et al. (2000b)) we approximate $E_{N}$ by $E_{1}$ for all sizes $N$ of interest.

Reasonable agreement between the calculated and the observed average resonance positions is observed for the choice $U_{0}=-0.5 \mathrm{eV}$ at low $N$ and $U_{0}=-0.6 \mathrm{eV}$ at higher $N$. With the weakly $N$-dependent choice (Stampfli, 1995)

$$
U_{0}(N)=a N^{-1 / 3}+b,
$$

using $a=+0.7(0.8) \mathrm{eV}$ for $N=q(N=q+1)$ and $b=-0.866 \mathrm{eV}$ in both cases, the full curves (labeled var. in (a) and (b)) are obtained, showing good agreement with the experimentally found binding energies over a broad range of $N$. The combined information contained in the $q$-dependent redshifts, in the respective resonance widths, in the calculated absolute values of the electron binding energies and in the slopes of the calculated $E_{\mathrm{B}}(N)$ and of the experimental $E_{\mathrm{R}}(q)$ curves allows the conclusion that the main contributions to cluster anion formation stem from neutral clusters with sizes $N=q$ and/or $N=q+1$. Note that in view of the rather narrow width of the observed VFRs a particular cluster anion size $q$ cannot be associated with a broad range of precursor sizes $N$ in view of the differential redshift of about $12 \mathrm{meV}$ per added $\mathrm{CO}_{2}$ unit.

The substantially larger binding energies observed for the VFRs in $\mathrm{CO}_{2}$ clusters as compared to those for the VFRs in $\mathrm{N}_{2} \mathrm{O}$ clusters (see Section C.2) are due to the fact that the short-range interaction $U_{0}$ for $\mathrm{CO}_{2}$ is attractive (note that the polarizabilities of $\mathrm{N}_{2} \mathrm{O}$ and $\mathrm{CO}_{2}$ agree to within $5 \%$ ). This conclusion is confirmed by analysis of experimental results and theoretical calculations of low-energy electron scattering by $\mathrm{CO}_{2}$ and $\mathrm{N}_{2} \mathrm{O}$ molecules. Cross-sections for low-energy electron scattering by $\mathrm{CO}_{2}$ are rapidly 
increasing towards lower energies (Morrison et al., 1982; Field et al., 2001b) approaching $200 \times 10^{-20} \mathrm{~m}^{2}$ which corresponds to a large negative scattering length whose value lies between $-7.2 \mathrm{a}_{0}$ and $-6.3 \mathrm{a}_{0}$ (Fabrikant, 1984; Mazevet et al., 2001; Field et al., 2001b). On the other hand, experimental (Szmytkowski et al., 1984) and theoretical (Sarpal et al., 1996; Winstead and McKoy, 1998) data on electron scattering by the $\mathrm{N}_{2} \mathrm{O}$ molecule show that the low-energy cross-section in this case is much smaller and does not exceed $8 \times 10^{-20} \mathrm{~m}^{2}$ below $E=1 \mathrm{eV}$. At ultralow energies, the cross-section should increase due to the (albeit very small, $\mu=0.16 \mathrm{D}$ ) dipole moment; but this effect should not be important for clusters. Note that the quadrupole moment of the molecule, although relevant, does not affect the energy dependence of the cross-section at low energies (Fabrikant, 1984; Leber et al., 2000b). For the clusters of interest here, the effects of the molecular dipole and quadrupole moments should be small as a result of cancellation due to mixed orientations. Therefore the observed redshifts of the VFRs can be reproduced by modelling the $\mathrm{e}^{-}-\mathrm{CO}_{2}$ and $\mathrm{e}^{-}-\mathrm{N}_{2} \mathrm{O}$ interaction potentials as a well or a repulsive barrier with a polarization tail. In the more general expression (44) for the short-range contribution to the electron interaction with clusters the parameter $b$ is the excess energy of the electron in the infinite medium and the factor $a$ is a constant which depends on the dielectric properties of the cluster. Our estimate of $b$ which employs the effective potentials of monomers and the concept of the Wigner-Seitz cell (Stampfli, 1995) shows that $b$ for bulk $\mathrm{N}_{2} \mathrm{O}$ exceeds the value of $b$ for bulk $\mathrm{CO}_{2}$ by $1 \mathrm{eV}$ which agrees well with our empirical values for $b$ of $-0.87 \mathrm{eV}$ for $\mathrm{CO}_{2}$ and about $+0.2 \mathrm{eV}$ for $\mathrm{N}_{2} \mathrm{O}$ (Leber et al., 2000c). The described model is of course very simple. For a detailed understanding of these fascinating vibrational resonances large-scale ab initio calculations are needed.

\section{C.4. Carbonyl Sulfide Clusters}

Carbonyl sulfide OCS in its ground electronic state is a linear molecule with an electric dipole moment of $\mu=0.715 \mathrm{D}$ (Gutsev et al., 1998). The lowest vibrationally excited states, labelled $\left(v_{1} v_{2} v_{3}\right)$, include the bending mode (010) $(64.5 \mathrm{meV})$, the asymmetric stretch mode $(001)(106.5 \mathrm{meV})$ and the bending overtone $(020)(129.0 \mathrm{meV})$. The ground state of the anion $\mathrm{OCS}^{-}$is bent with an angle of $136^{\circ}$. The adiabatic electron affinity $(A E A)$ of OCS is not yet well known. Gutsev et al. (1998) report a calculated value of $A E A(\mathrm{OCS})=-0.22 \mathrm{eV}(\mathrm{CCSD}(\mathrm{T})$ method), while Surber et al. (2002) conclude that $A E A(\mathrm{OCS})$ is close to zero. Values of this magnitude are supported by the fact that the properties of OCS are intermediate between those of $\mathrm{CO}_{2}$ and $\mathrm{CS}_{2}$ which have adiabatic electron affinities 
of $A E A\left(\mathrm{CO}_{2}\right) \approx-0.66 \mathrm{eV}$ (Gutsev et al., 1998) and $A E A\left(\mathrm{CS}_{2}\right)=0.9-1.0 \mathrm{eV}$ (Oakes and Ellison, 1986). The theoretical results for $A E A(\mathrm{OCS})$, however, differ from the experimental value of $A E A(\mathrm{OCS}) \approx 0.4 \mathrm{eV}$ (Compton et al., 1975; Chen and Wentworth, 1983).

The dissociation energy of OCS towards $\mathrm{S}+\mathrm{CO}$ amounts to $D_{0}(\mathrm{OC}-\mathrm{S})=$ $3.13(4) \mathrm{eV}$ (Ziesel et al., 1975b) and the electron affinity of $\mathrm{S}$ to $E A(\mathrm{~S})=$ $2.0772 \mathrm{eV}$ (Andersen et al., 1999). $\mathrm{S}^{-}$formation in dissociative attachment to OCS is thus accessible at energies above about $1.05 \mathrm{eV}$ in agreement with experimental findings (Ziesel et al., 1975b; Iga et al., 1996). which show a prominent peak for $\mathrm{S}^{-}$formation, centered at about $1.35 \mathrm{eV}$ (cross-section of about $2.7 \times 10^{-21} \mathrm{~m}^{2}$ ). This peak is connected with a resonance peak found at about $1.15 \mathrm{eV}$ in elastic scattering from OCS (Karwacz et al., 2001a).

Rather little is known up to now on the formation of carbonyl sulfide cluster anions and on their geometrical structure. Kondow and Mitsuke (1985) investigated formation of negative cluster ions of OCS produced by electron transfer from a range of Rydberg rare gas atoms around $n=25$. Their mass spectrum is dominated by (OCS $)_{q}^{-}$ions with a maximum at $q=10$. To our knowledge no free electron attachment spectra for OCS clusters have been reported prior to the LPA work discussed below. Regarding cluster anion spectroscopy and structure, Sanov et al. (1998) studied the photochemistry of (OCS) $)_{n}^{-}$cluster ions following excitation by $395 \mathrm{~nm}$ and $790 \mathrm{~nm}$ photons. They also presented possible equilibrium geometries of $(\mathrm{OCS})_{2}^{-}$and the relative potential energy curves for the neutral dimer $(\mathrm{OCS})_{2}$ and the anion $(\mathrm{OCS})_{2}^{-}$.

Using the laser photoelectron attachment method, Barsotti et al. (2003b) have recently studied the formation of cluster anions in RET and in lowenergy free electron attachment $(E=1-200 \mathrm{meV})$ to molecular clusters of carbonyl sulfide OCS at energy widths of 1-2 meV. In Fig. 48 the intensities, for positive $(\mathrm{OCS})_{q}^{+}$ions (due to $85 \mathrm{eV}$ electron impact) and normalized to the anion signal at $q=2$ and for negative (OCS) $q$ ions (resulting from RET at high $n \approx 260$ ) are compared. The anion mass spectrum shows a clear peak at the dimer, but little structure otherwise.

The energy dependences for $(\mathrm{OCS})_{q}^{-}$anion formation by free electron attachment, shown in Fig. 49 for $q=1-9$, are characterized by a strong rise towards zero energy (attributed to s-wave attachment) as well as by vibrational Feshbach resonances (VFR) whose importance decreases towards larger cluster sizes $q$. The electron attachment behavior of OCS clusters is thus intermediate between that of $\mathrm{CO}_{2}$ clusters (which is dominated by VFRs) and that of $\mathrm{CS}_{2}$ (which exhibits a strong zero-energy peak, but no VFRs, see Section C5). Formation of the (OCS) $)_{2}^{-}$dimer anion is especially enhanced; its attachment spectrum exhibits a sharp vibrational 


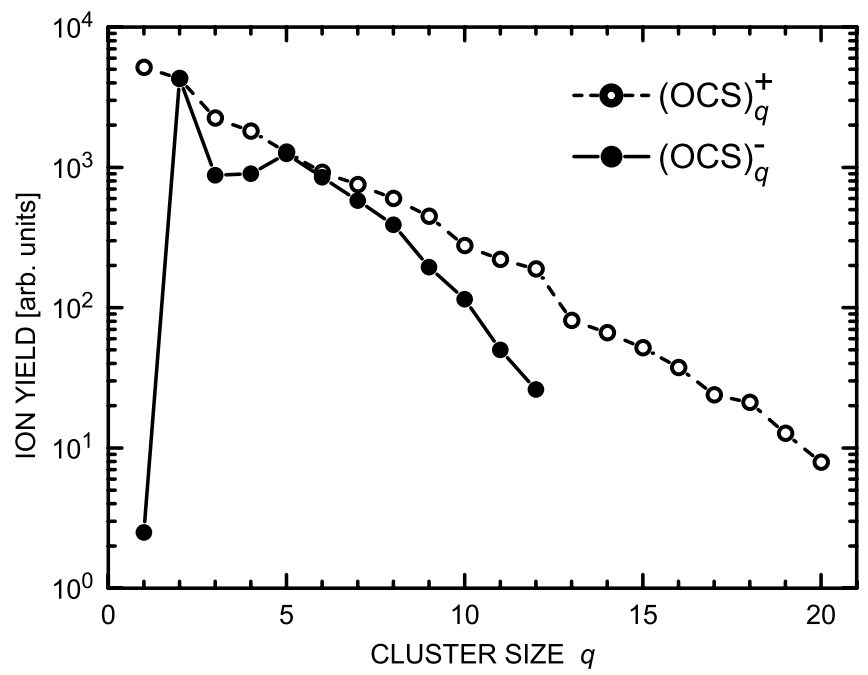

FIG. 48. Intensities of homogenous positive/negative ions (normalized at $q=2$ ) due to $85 \mathrm{eV}$ electron impact/threshold electron attachment involving OCS clusters (Barsotti et al., 2003b).

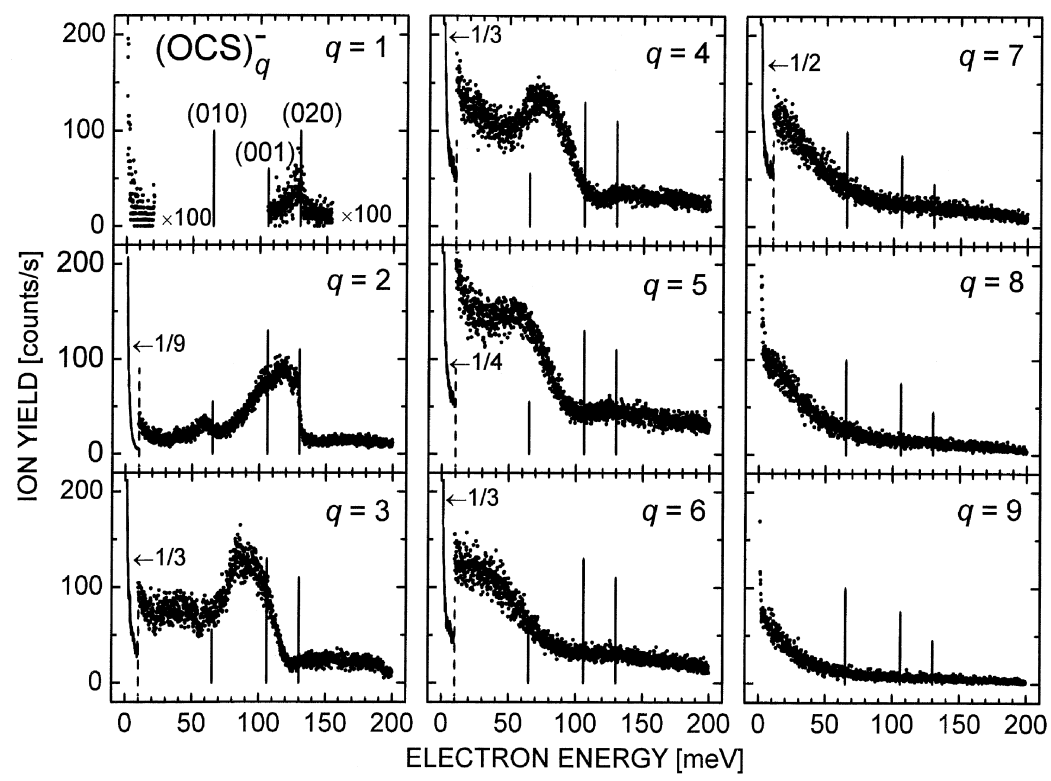

FIG. 49. Low-energy electron attachment spectra for formation of (OCS $)_{q}^{-}$anions from $(\mathrm{OCS})_{N}$ clusters $(q \leq N)$. The full vertical lines denote the energy positions of the listed vibrational modes in the OCS monomer (from Barsotti et al., 2003b). 
Feshbach resonance at $128 \mathrm{meV}$, just below the (020) onset for excitation of the $\left(v_{1} v_{2} v_{3}\right)$ normal modes in the OCS molecule, a broad structure centered at about $117 \mathrm{meV}$, a weak structure at about $103 \mathrm{meV}$ (just below the $v_{3}=1$ onset) and a peak at about $59 \mathrm{meV}$ below the $v_{2}=1$ onset. The attachment spectrum observed for $q=1$ shows a low counting rate; it also exhibits a rather sharp resonance peaking at about $128 \mathrm{meV}$. The low intensity may indicate that $\mathrm{OCS}^{-}$only exists in a few stable or even metastable rovibrational states, in line with the theoretical results of Surber et al. (2002). Since the spectra for $q=1$ and $q=2$ both exhibit the (020)-peak essentially at the same energy position, it is plausible that the yield for the monomer anion $\mathrm{OCS}^{-}$is due to formation of the dimer (OCS) $)_{2}^{-}$with subsequent evaporation of a single OCS unit (and not due to attachment of clusters with sizes $q \geq 3$; it is also not expected that (long-lived) $\mathrm{OCS}^{-}$anions are formed through free electron attachment to OCS monomers).

For $q=3$ the very sharp peak present in the spectrum at $q=2$ and assigned to the vibrational Feshbach resonance of the type $v_{2}=2$ is no longer observed, the broad structure appears red-shifted by about $20 \mathrm{meV}$, and a peak at lower energies is weakly observed, also red-shifted by about $20 \mathrm{meV}$. With the increase of the cluster size $(4 \leq q \leq 7)$ only a broad structure is left in the spectra, red-shifted by about $20 \mathrm{meV}$ per added OCS unit. For $q>8$ no clear structure is left in the spectra which are dominated by the rise towards zero energy. The missing of the sharp resonance for $q>2$ is similar to the findings for electron attachment to methyl iodide clusters (Weber et al., 2000, see also subsection C.6). The sharp vibrational Feshbach resonance, observed at about $61 \mathrm{meV}$ (just below the onset for the $\mathrm{C}-\mathrm{I}$ stretch vibration $v_{3}=1$ ) for dissociative attachment to $\mathrm{CH}_{3} \mathrm{I}$ monomers (yielding $\mathrm{I}^{-}$ions), is almost missing in attachment spectra for $\left(\mathrm{CH}_{3} \mathrm{I}\right) \mathrm{I}^{-}$and $\left(\mathrm{CH}_{3} \mathrm{I}\right)_{2} \mathrm{I}^{-}$, which weakly exhibit broad red-shifted structure.

\section{C.5. Carbon Disulfide Clusters}

Carbon disulfide $\mathrm{CS}_{2}$ is linear in its electronic ground state. According to ab initio calculations (Gutsev et al., 1998) the adiabatic electron affinity is positive with a value of $E A_{\text {ad }}=0.30 \mathrm{eV}$; the stable anion is bent with a bond angle of about $144.5^{\circ}$ and an electric dipole moment of $+0.46 \mathrm{D}$. The difference between the theoretical value for $E A_{\text {ad }}$ and the experimental result $(0.89 \mathrm{eV}$, Oakes and Ellison, 1986) might in part be due to very unfavourable Franck-Condon-factors for the transition connecting the respective vibrational ground levels from the anion to the neutral, precluding observation of this transition in the experiment (Gutsev et al., 1998). For optimized linear configurations of $\mathrm{CS}_{2}$ and $\mathrm{CS}_{2}^{-}$, their energies are almost identical with the possibility that the linear anion is stable against 


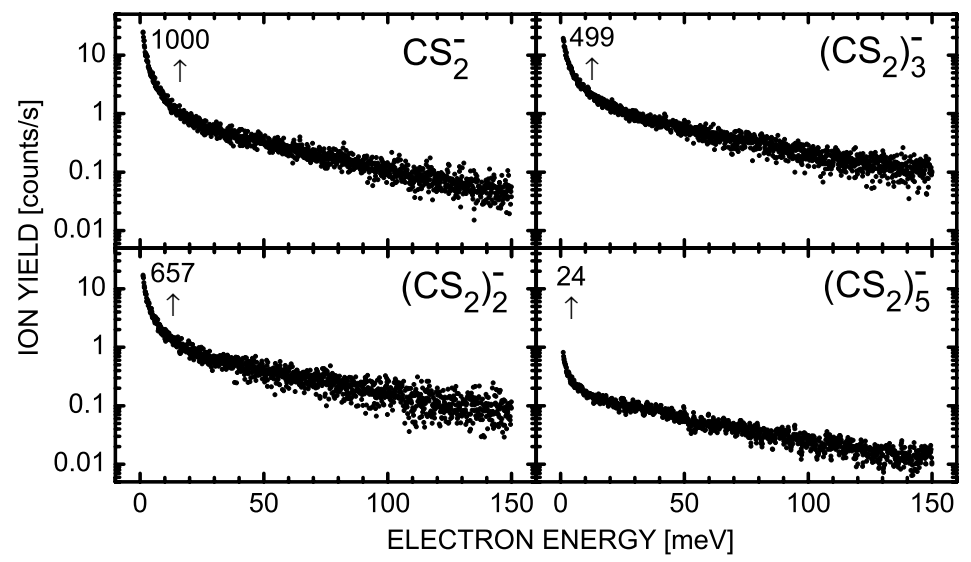

FIG. 50. Low-energy electron attachment spectra for formation of $\left(\mathrm{CS}_{2}\right)_{q}{ }^{-}$anions from $\left(\mathrm{CS}_{2}\right)_{N}$ clusters $(N \geq q=1,2,3,5)$. The numbers above the arrows near zero energy give the cluster anion yields for RET at $n \approx 260$ (from Barsotti et al., 2002a).

autodetachment by a few millielectron volts. This finding is compatible with substantial rate coefficients for the formation of $\mathrm{CS}_{2}^{-}$ions in RET experiments (Kalamarides et al., 1988; Harth et al., 1989; Carman et al., 1990) and can explain observations of long-lived weakly bound $\mathrm{CS}_{2}^{-}$ions which undergo electric-field-induced detachment when subjected to fields of only a few kilovolts per centimeter (Kalamarides et al., 1988). Anion formation involving carbon disulfide clusters has been studied by RET (Kondow, 1987; Desfrançois et al., 1993) and, more recently, in free electron attachment experiments with the LPA method (Barsotti et al., 2002a). Photodetachment and photodestruction studies of $\left(\mathrm{CS}_{2}\right)_{q}^{-}$cluster anions (e.g. Maeyama et al., 1998) in conjunction with ab initio calculations of the anion structures and binding energies (e.g. Sanov et al., 1998) indicate that a $\mathrm{C}_{2} \mathrm{~S}_{4}^{-}$core is involved in the $\left(\mathrm{CS}_{2}\right)_{q}^{-}$cluster anions with $q \geq 2$.

Figure 51 presents the attachment spectra for formation of small carbon disulfide cluster anions with sizes $q=1,2,3,5$, as measured with the LPA method over the energy range $1-150 \mathrm{meV}$ (Barsotti et al., 2002a); $\mathrm{CS}_{2}$ molecules $(0.34$ bar) were coexpanded with helium carrier gas at a total stagnation pressure of 4.5 bar). For all cluster anion sizes, the attachment yield shows a monotonous decline towards higher electron energies. Within the statistical uncertainties no structure is observed. The anion cluster size distribution in the free electron attachment spectra, viewed at any particular electron energy, was found to be close to that observed in the threshold attachment RET mass spectrum which decreased monotonically with 


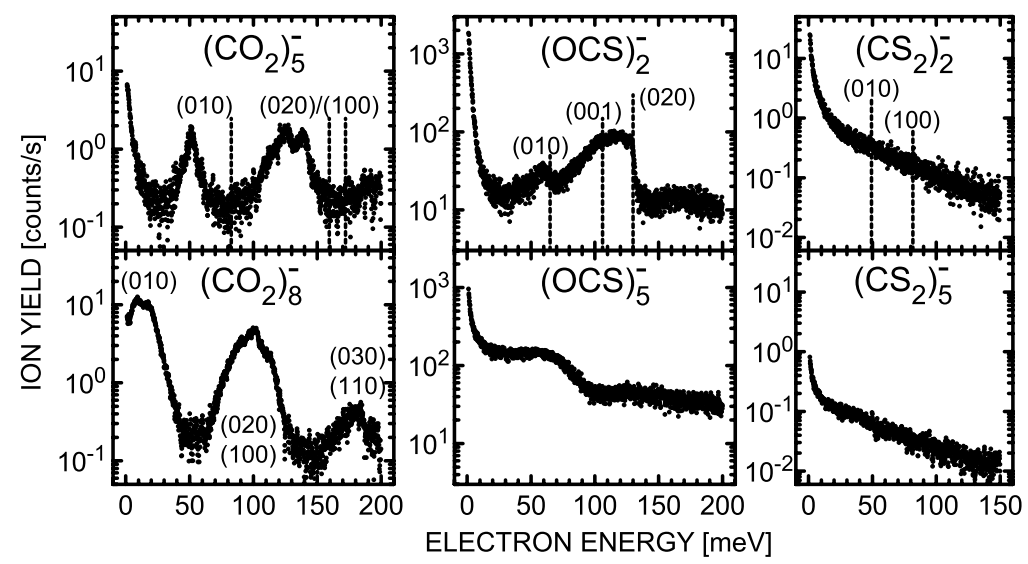

FIG. 51. Comparison of attachment spectra for formation of homogenous cluster anions $\left(\mathrm{CO}_{2}\right)_{q}(q=5,8),(\mathrm{OCS})_{q}(q=2,5)$, and $\left(\mathrm{CS}_{2}\right)_{q}(q=2,5)$. Vibrational Feshbach resonance dominate the spectra for $\left(\mathrm{CO}_{2}\right)_{q}$ formation and are still prominent (though broader) in the $(\mathrm{OCS})_{q}$ spectra (from Barsotti et al., 2002a, 2003b).

rising $q \geq 1$. Electron attachment at near-zero energies is efficient for any size (including the monomer); the formed temporary cluster anion may evaporate monomers at an energy expense of about $0.17 \mathrm{eV}$ per molecule (Desfrançois et al., 1993). In this way reaction (42c) can proceed with an evaporation of one or more monomers (depending on the values of the respective electron affinities and evaporation energies). In collisions with free electrons or Rydberg electrons with sufficiently high principal quantum number long-lived monomer $\mathrm{CS}_{2}^{-}$anions are not formed from the neutral monomer; in these cases, the yield for $\mathrm{CS}_{2}^{-}$formation originates from neutral clusters with sizes $N \geq 2$.

In comparison with the observations made for cluster anion formation from $\mathrm{CO}_{2}$ and OCS clusters, in particular with regard to the presence of vibrational resonances, (see Fig. 1) the question arises why no vibrational structure is observed at all for carbon disulfide clusters. Note that 'giant' scattering resonances have been observed in the energy dependence of the total cross-section (Jones et al., 2002) as well as in the angle differential elastic cross-section (Allan, 2001c) for electron scattering from $\mathrm{CS}_{2}$ molecules at very low energies, as discussed in Section B.6 of this chapter. One essential difference between $\mathrm{CO}_{2}, \mathrm{OCS}$ and $\mathrm{CS}_{2}$ is the substantial rise in the adiabatic (as well as in the vertical) electron affinity from $\mathrm{CO}_{2}$ to $\mathrm{CS}_{2}$. Carbon dioxide does not form a stable negative ion, OCS anions are just about stable while $\mathrm{CS}_{2}$ possesses states bound by several tenths of an $\mathrm{eV}$ 
(see above). For $\mathrm{CS}_{2}$ clusters the stronger valence-type electron binding and the expected stronger effects due to solvation in the cluster anions may thus preclude the formation of VFRs which reflect long-range electron binding.

\section{C.6. Methyl Iodide Clusters}

As described in Section IV.A, a prominent VFR has been observed (Schramm et al., 1999) in dissociative attachment to $\mathrm{CH}_{3} \mathrm{I}$ molecules (yielding $\mathrm{I}^{-}$ions) directly below the onset for the $v_{3}=1 \mathrm{C}-\mathrm{I}$ stretch vibration. It was shown that the VFR occurs due to the combined effects of the dipole and the polarization attraction, and it may thus be expected that this VFR will look quite different in clusters of methyl iodide, if present at all. We note that the $v_{3}=1 \mathrm{VFR}$ has been suggested to play a role in photofragmentation of the $\mathrm{I}^{-} \cdot \mathrm{CH}_{3} \mathrm{I}$ anion yielding $\mathrm{I}^{-}$ions (Dessent et al., 1996).

The cross-sections for $\left(\mathrm{CH}_{3} \mathrm{I}\right)_{q} \mathrm{I}^{-}(q=1,2)$ cluster anion formation reveal in fact a dramatic influence of the cluster environment on the VFR observed for methyl iodide monomers: for $q=1$ a weak, broad shoulder, shifted to lower energy, remains while for $q=2$, no significant structure is observed. This behavior was attributed to solvation effects which move the resonance position to lower energies and substantially increase the resonance width. In Fig. 52 we show calculated cross-sections for different solvation energies (but for a fixed polarizability) using a modified $R$-matrix model (Weber et al., 2000).

However, the cusp structure associated with the threshold for vibrational excitation of the symmetric C-I stretch at $E=66.1 \mathrm{meV}$ is still clearly seen in Fig. 52, whereas experimental data do not exhibit the cusp. This can be explained by interaction of the $\mathrm{C}-\mathrm{I}$ stretch mode with other modes in the complex. In particular, the vibrational dynamics of the dimer is influenced by interaction between the I atoms. To account for this effect, a model (Thoss and Domcke, 1998) describing interaction between a specific (system) vibrational mode with a background (bath) mode was adopted in Weber et al. (2000). The background mode was described by the displaced harmonic oscillator model (Domcke and Cederbaum, 1977). This approach allows to construct the Green's function describing both $\mathrm{C}-\mathrm{I}$ and soft-mode vibrations. After calculation of its matrix elements, the problem is reduced to solving a system of $N_{\mathrm{v}} \times N_{\mathrm{p}}$ linear algebraic equations for $N_{\mathrm{v}} \times N_{\mathrm{p}}$ attachment amplitudes, where $N_{\mathrm{v}}$ is the number of states describing C-I stretch vibrations, and $N_{\mathrm{p}}$ the number of states describing the soft-mode vibrations. The bath mode is characterized by the frequency parameter $\omega$ and the coupling parameter $\Theta=(1 / 2) \mu \omega R_{0}^{2}$ where $\mu$ is the reduced mass corresponding to soft-mode vibrations and $R_{0}$ is the distance between the 


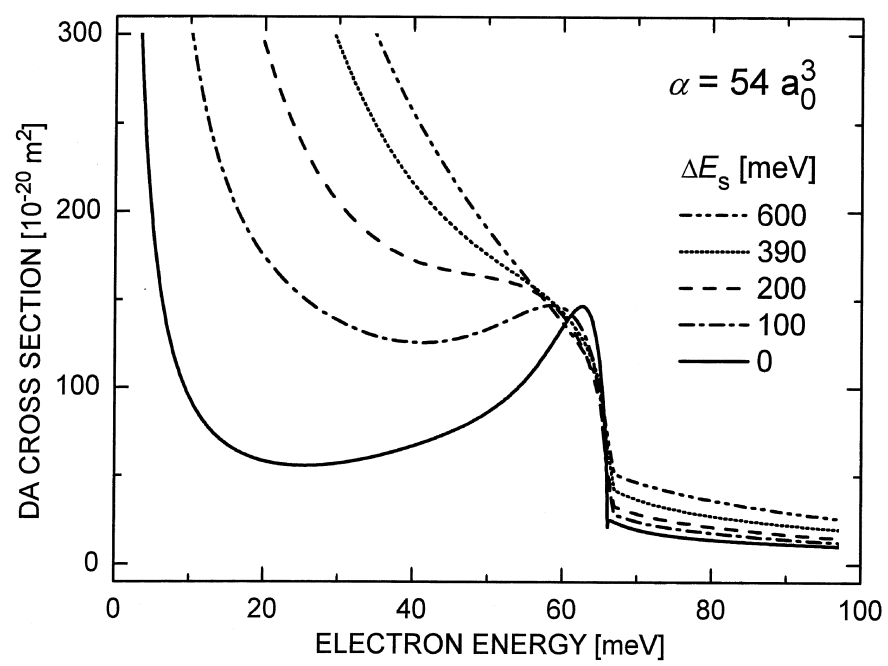

FIG. 52. Calculated cross-sections for dissociative attachment to $\mathrm{CH}_{3} \mathrm{I}$ monomers with potential curves, "solvation"-shifted by the indicated amounts (from Weber et al., 2000).

minima of the two porential curves describing the neutral molecule and the negative ion. Another effect which was included in the calculations is an additional polarization interaction for the incident electron due to the presence of the second molecule in the dimer. Some sample results for the DA rate coefficients and their comparison with experiment are presented in Fig. 53.

Agreement between theory and experiment should be considered more qualitative than quantitative at the present stage: the theory confirms that the solvation and polarization effects destroy the vibrational Feshbach resonance and lead to almost complete disappearance of the threshold cusp due to coupling with the bath mode. On the other hand, the theoretical rate coefficient drops with energy too fast as compared to the experimental observations. One of the possible reasons for this is an overestimation of the polarization energy in the theoretical model. Indeed, calculations with a polarization potential whose strength is reduced at shorter distances lead to a better agreement with experiment, as we can see from Fig. 53.

\section{Related Topic: Positron Annihilation}

Although this review is focused on electron-molecule collisions, a related topic of positron-molecule collisions is important and will be briefly 

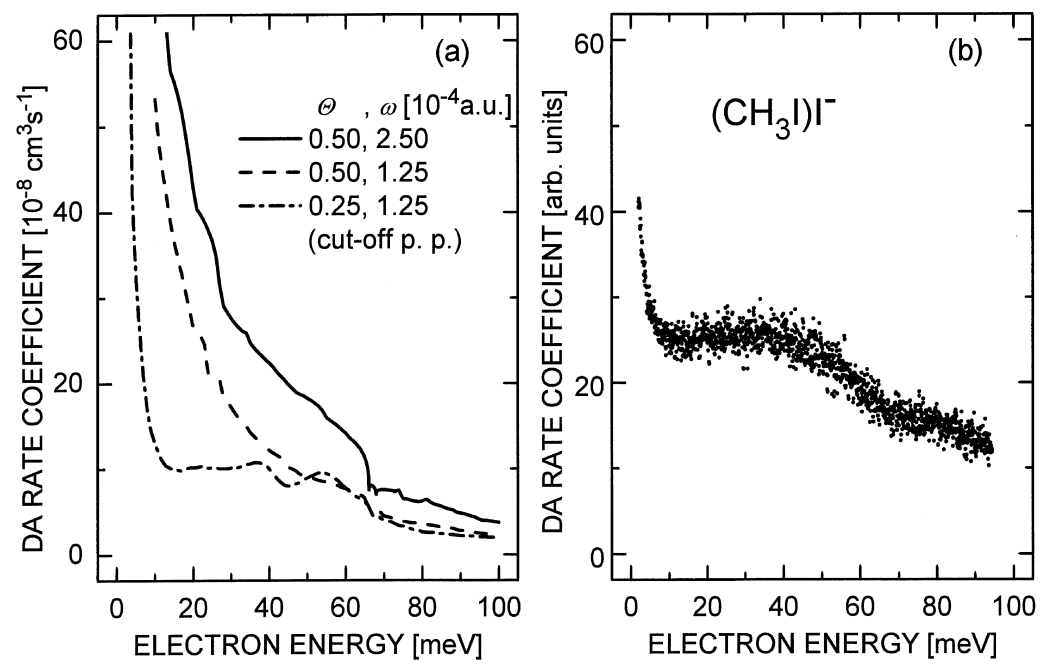

FIG. 53. Comparison between (a) calculated and (b) experimental rate coefficients for dissociative attachment to methyl iodide dimers yielding $\left(\mathrm{CH}_{3} \mathrm{I}\right) \mathrm{I}^{-}$ions. The theoretical curves correspond to different choices of the bath-mode parameters. The chain curve represents a model with a reduced polarization interaction (from Weber et al., 2000).

discussed here. Resonances in positron collisions with atomic and molecular systems were predicted in several theoretical works (for a more complete review of the subject the reader is referred to the paper of Kimura et al. (2000)), however until recently there was no direct experimental evidence for them. Indirect evidence for these resonances was obtained in 1963 when Paul and Saint-Pierre (1963) observed very high annihilation rates in thermal positron collisions with certain large molecules. Surko et al. (1988) extended these studies to larger organic molecules. More recently these findings were confirmed by a series of experiments performed at the University of San Diego (Murphy and Surko, 1991; Iwata et al., 1995; Iwata et al., 2000).

The annihilation rate is usually characterized by the parameter

$$
Z_{\text {eff }}=\lambda\left(\pi r_{0}^{2} c n\right)^{-1},
$$

where $\lambda$ is the observed annihilation rate, $r_{0}$ is the classical electron radius, $c$ is the speed of light, and $n$ is the molecule number density. Equation (45) reflects a naive view of the annihilation process according to which the annihilation rate can be obtained by multiplication of the Dirac annihilation 
rate of positronium (Ps) by an effective number $Z_{\text {eff }}$ of electrons in the target molecule. However measurements for many hydrocarbons and partially fluorinated hydrocarbons yield values of $Z_{\text {eff }}$ which exceed the actual number of electrons by several (sometimes 4 or even 5) orders of magnitude. This is particularly surprising because the measurements are pertinent to positron energies below the positronium formation threshold. However, Murphy and Surko (1991) found a simple (albeit pure empirical) scaling relation for all studied atoms and single-bonded nonpolar molecules.

$$
\ln \left(Z_{\text {eff }}\right)=A\left(E_{\mathrm{i}}-E_{\mathrm{Ps}}\right)^{-1},
$$

where $E_{\mathrm{i}}$ is the ionization energy of the target and $E_{\mathrm{Ps}}$ is the binding energy of positronium. Other interesting observations are: (i) the annihilation rates for the deuterated and protonated alkanes are very similar if not identical at room temperatures; (ii) singly fluorinated hydrocarbons have even higher annihilation rates whereas further fluorination leads to a decrease of annihilation rate with the perfluorinated molecule having the lowest rate; (iii) there is no strong correlation between $Z_{\text {eff }}$ and molecular dipole moment.

A theoretical model (Gribakin, 2000, 2001; Iwata et al., 2000) with two regimes was proposed to explain the observed rates. According to the first mechanism, annihilation enhancement can occur due to a weakly bound or a virtual state in the positron-molecule complex. This mechanism (direct annihilation) can explain a moderate $\left(Z_{\text {eff }}\right.$ below about 1000$)$ enhancement of the rate. For higher rates a resonant mechanism associated with a temporary capture of the positron into the field of vibrationally excited molecules (VFR) is introduced.

The influence of VFRs on the annihilation rates was recently confirmed by experiments (Gilbert et al., 2002; Barnes et al., 2003) with positron beams whereby Ps annihilation rates were investigated as a function of the positron energies from $50 \mathrm{meV}$ to several $\mathrm{eV}$ at an energy width of about $25 \mathrm{meV}$. Pronounced peaks below several vibrational excitation thresholds have been observed providing the first direct evidence for long-lived vibrational resonances of the positron-molecule complex. Two examples are shown in Fig. 54. The redshifts of the vibrational resonances (relative to the position of the $\mathrm{C}-\mathrm{H}$ stretch mode, dashed line) amount to $0.03 \mathrm{eV}$ in propane and $0.13 \mathrm{eV}$ in heptane; they are interpreted as a measure of the positronmolecule binding energy (see Barnes et al., 2003 for details).

The existence of VFRs in positron-molecule systems suggests that there should be bound states of positron-molecule systems. However, very little 

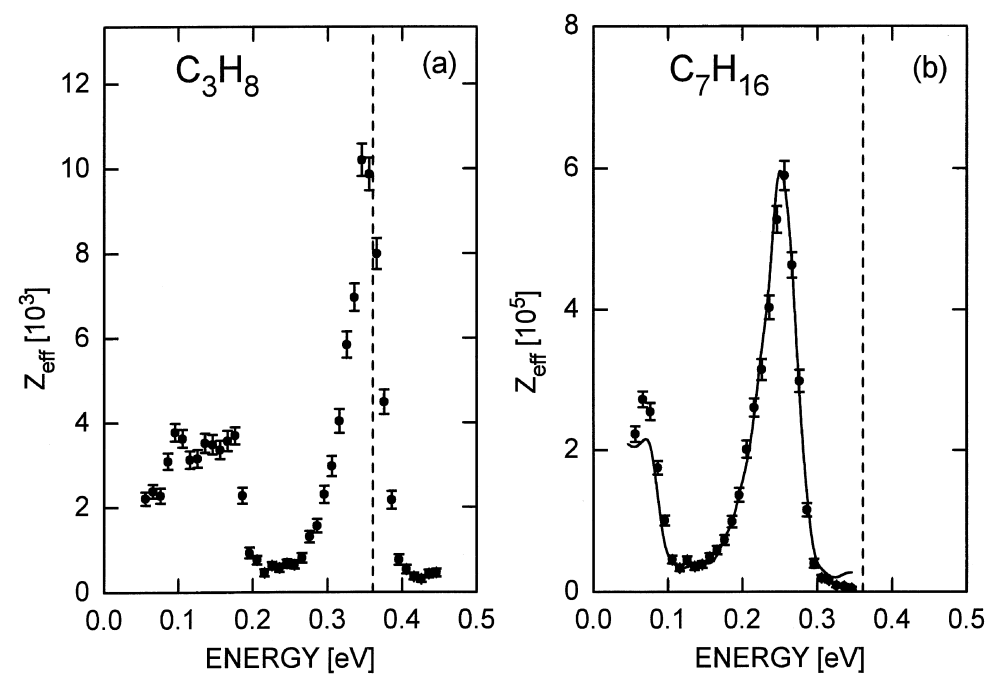

FIG. 54. Energy-resolved annihilation rates, $Z_{\text {eff }}$, for (a) propane and (b) heptane. For comparison, the solid line in (b) shows $Z_{\text {eff }}$ for propane, scaled by 60 and shifted downward in energy by $0.1 \mathrm{eV}$ (Barnes et al., 2003).

is known about such systems. Even for positron-atom bound states, calculations became possible only recently (Dzuba et al., 1995; Ryzhikh and Mitroy, 1997), and virtually nothing is known about molecules, although some results were obtained for the relation between positron affinities and molecular dipole moments (Tachikawa et al., 2001). However, experimental data do not indicate any significance of the dipole moment for enhanced annihilation. Theoretical calculations of positron affinities of molecules are particularly difficult because of the relative weakness of positron-molecule interactions as compared to the electron-molecule interaction. The reason is simple: the static potential for the positron-molecule interaction is repulsive and therefore it strongly reduces the attraction due to the polarization interaction (Kimura et al., 2000). This makes inclusion of positron-electron correlations especially important, therefore calculations of positron affinities are more difficult than those of electron affinities.

Another theoretical challenge has to do with the density of VFRs. According to recent work on positron annihilation rates (Gribakin, 2000, 2001), a high density of VFRs, growing exponentially with the number of atoms in the molecule, is required in order to explain the measured annihilation rates. This was confirmed by recent experiments of Barnes et al. (2003) which indicate a strong correlation between the spectrum of 
vibrational modes and the annihilation spectrum for propane and heptane (see Fig. 54). Although we can expect that VFRs are a quite common phenomenon in electron and positron scattering by polyatomic systems, there are no theoretical calculations to confirm this judgement. Finally, studies of VFRs in electron-molecule scattering show that VFRs are typically supported by the long-range field of the molecule, whereas annihilation data do not exhibit any correlation between the observed rates and molecular dipole moments. Certainly, more experimental and theoretical studies are required to explain this interesting phenomenon. The theory is still in a very preliminary stage at this point. No calculations of VFRs and corresponding annihilation rates are available even for simple diatomic molecules.

However, one aspect of the theory, namely the behavior of the annihilation cross-section near the threshold for positronium formation, has been studied in some detail. Equation (46) might suggest that the annihilation cross-section exhibits a singularity near the threshold. Indeed, according to the model of Laricchia and Wilkin (1997), $Z_{\text {eff }}$ grows as $\left|E_{\mathrm{i}}-E_{\mathrm{Ps}}\right|^{-1}$. Variational calculations (Van Reeth and Humberston, 1998) demonstrate a weaker divergence $\left|E_{\mathrm{i}}-E_{\mathrm{Ps}}\right|^{-1 / 2}$. However, any divergence would violate unitarity of the $S$ matrix. To resolve this controversy one has to introduce the coupling between the Ps formation channel and the annihilation channel (Gribakin and Ludlow 2002; Igarashi et al., 2002). Due to this coupling the Ps formation channel is blurred by the annihilation energy width, and the $\left|E_{\mathrm{i}}-E_{\mathrm{Ps}}\right|^{-1 / 2}$ singularity is removed. Recent calculations (Gribakin and Ludlow, 2002; Igarashi et al., 2002) for annihilation in $\mathrm{e}^{+}-\mathrm{H}$ collisions demonstrate that the cross-section behaves as a continuous function of positron energy near the Ps formation threshold. This result actually follows from a general theory of threshold behavior for creation of an unstable particle (Baz', 1961).

\section{Conclusions and Perspectives}

As shown in this review, significant progress has been made over the past decade in our understanding of resonance and threshold phenomena in low-energy electron interactions with molecules and clusters. At very low energies (below about $0.2 \mathrm{eV}$ ) novel photoelectron techniques have allowed to study attachment cross-sections for both molecules and molecular clusters at unprecedented (sub-) $\mathrm{meV}$ resolution and down to sub- $\mathrm{meV}$ energies. Sharp vibrational Feshbach resonances have been discovered in molecules as well as molecular clusters, and the fundamental threshold behaviour was convincingly demonstrated for several polar and nonpolar 
molecules. With free electron cross-sections measured to sub- meV energies, a realistic extrapolation to zero energy became possible, and accurate absolute attachment cross-sections were established with reference to reliable thermal rate coefficients due to electron swarm experiments. For the first time a critical comparison between free electron attachment and Rydberg electron transfer data became possible by calculating - within the quasi-free electron model for Rydberg electron collisions - the RET rate coefficient from measured free electron attachment cross-sections, and good agreement was observed for several molecules (Klar et al., 1994b, 2001a, b; Dunning, 1995).

Using synchrotron-based VUV photoelectron sources, measurements of total cross-sections have been extended down to about $10 \mathrm{meV}$. Strong resonances have been detected in $\mathrm{CS}_{2}$ which are similarly present in the differential elastic cross-section. It can be expected that these observations are just the beginning of an exciting future of 'cold electron collisions' (Field et al., 2001a) with many resonances yet to discover in electron scattering and attachment cross-sections. Intriguing examples of such features are the observation of a deep dip in the total scattering cross-section at $0.1 \mathrm{eV}$ for nitrobenzene (Lunt et al., 2001) and at $0.07 \mathrm{eV}$ for the $\mathrm{CF}_{3} \mathrm{Cl}$ molecule (Field et al., 2001c), tentatively ascribed to interference between a direct channel of rotational excitation and indirect excitation via a short-lived negative ion state. Their observation and explanation poses a challenge to theory.

Substantial progress has also been made in angle-differential electron scattering studies, using optimized and well-calibrated electrostatic spectrometers at energy widths below $10 \mathrm{meV}$. Theoretical ab initio DA and VE cross-sections for hydrogen halides, obtained with the nonlocal resonance model, agree very well with accurate measurements, and, what is even more important, many features observed there, like threshold peaks, boomerang oscillations and VFRs, are now well understood. However, for several other diatomic molecules the situation is not as clear. In important cases such as chlorine, no reliable experimental cross-sections for vibrational excitation are available, and $R$-matrix calculations of VE and DA cross-sections in part had to use semiempirical input. A fully ab initio calculation of VE and DA involving fluorine molecules has been carried out, but the controversy related to the low-energy behavior of DA (p-wave versus s-wave) is still unresolved. Certainly more experimental results are needed here.

The situation with polyatomic molecules is even more complicated. We have achieved a qualitative understanding of some important features. The known cases suggest that threshold peaks and possibly also sharp structures due to VFR may be found in many, presumably most polyatomic 
molecules. They are linked to anion states with an electron in a spatially diffuse wave function, bound by a combination of dipolar and polarization forces; the dipolar forces may be active even in molecules without a permanent dipole moment (such as $\mathrm{CO}_{2}$ ) when the parent vibrational state of the VFR is not totally symmetric and acquires a nonzero dipole moment upon variation of its normal coordinate. The VFRs may substantially affect the cross-sections by acting as doorways into valence, possibly dissociative states. The outer wells in anion potentials and the associated outer well resonances described here are likely to represent prototype cases for outer wells in many polyatomic molecules. Here the electron is in a valence orbital whose antibonding properties are responsible for the substantially different geometry. More studies of polyatomic molecules at energies below about $1 \mathrm{eV}$ will be needed to confirm these expectations.

Completely $a b$ initio calculations of near-threshold and resonance processes for polyatomic molecules are still very difficult. In this field model and semiempirical calculations continue to play an important role. Approaches such as the resonance $R$-matrix method allow us to produce cross-sections for many transitions using just a few adjustable parameters. This method was particularly successful for our understanding of VFRs and threshold features in electron collisions with methyl halides. The major challenge in this field is extension of the existing methods towards incorporation of several vibrational modes. For this purpose the wavepacket propagation method (McCurdy and Turner, 1983; Kazansky, 1995) seems to be very promising. Some results have been recently obtained for dissociative attachment (Kazansky, 1995) and vibrational excitation (Rescigno et al., 2002) of the $\mathrm{CO}_{2}$ molecule. However, the split time propagation method has been implemented so far only within the framework of the local resonance model which is not able to describe correctly threshold phenomena and VFRs. A relevant example is dissociative attachment to the $\mathrm{CH}_{2} \mathrm{Br}_{2}$ molecule with the formation of $\mathrm{Br}^{-}$. This process cannot be described in a one vibrational mode approximation. On the other hand, a multidimensional description of the vibrational dynamics should also include nonlocal effects in order to reproduce the VFR observed below the threshold for excitation of the symmetric $\mathrm{C}-\mathrm{Br}$ stretch. Another challenging example is a recent discovery of a zero energy peak in $\mathrm{O}^{-}$formation due to dissociative electron attachment to ozone (Senn et al., 1999), which may play an important role in the destruction of ozone in the earth's atmosphere; it is still waiting for an explanation, also in view of the fact that thermal rate coefficients for electron attachment to ozone are not compatible with a zero energy resonance (van Doren et al., 2003). 
With the increasing complexity of the target, the situation is becoming even more challenging. In particular, we do not have a general theory of electron attachment to clusters, although some observed features in electron-cluster interactions, like VFRs and their dependence on cluster environment, have received an explanation recently. The resonance $R$-matrix theory has been generalized to describe DA to methyl iodide clusters. However, almost no calculations, except some preliminary results for $\mathrm{CO}_{2}$ clusters (Tsukada et al., 1987), were done for nondissociative attachment. The major remaining task here is the correct account for the coupling between the phonon modes in the cluster and the vibrational modes of individual molecules leading to VFRs in nondissociative attachment. Several controversies in low-energy electron attachment to $\mathrm{C}_{60}$ remain unresolved and demand further work.

Although not dealt with in this review, we mention metal clusters as another object for future studies. One-electron shape resonances in elastic scattering (Bernath et al., 1995; Ipatov et al., 1998a) and collective plasmon resonances in elastic and inelastic electron collisions with metal clusters (Gerchikov et al., 1998; Ipatov et al., 1998b; Connerade et al., 2000) have been predicted in theoretical calculations and wait for experimental confirmation. Measurements of electron capture by sodium clusters (Kasperovich et al., 2000a, b) indicate the validity of the Langevin formula for capture, as modified by employing the full image-charge interaction potential which accounts for the finite size of the cluster (Kasperovich et al., 2000b). Evidence for resonance-enhanced electron capture by potassium clusters has been reported by Sentürk et al. (2000). Another class of objects, attracting strongly rising interest recently, are biomolecules. One of the driving motivations is the question how radiation damage is induced by the interaction of slow (secondary) electrons with the constituents of biological systems. Here we just mention pioneering work by Boudaiffa et al. (2000) who reported resonant formation of DNA strand breaks by low-energy electrons.

Studies of vibrational resonances in positron-molecule collisions are only at the beginning stage. Their important observation in recent experiments has received only qualitative explanation so far, and many unanswered questions exist in this field. In particular we do not know yet why the annihilation rates are high in the thermal-energy region, well below the threshold for vibrational excitation. No calculations of electron affinities of molecular targets, supporting the assumption about VFRs in positronmolecule scattering, exist. The observed widths of VFRs in these processes are also awaiting for theoretical explanations.

Progress in future work on high-resolution low-energy electron (as well as positron) collisions will inevitably be bound to improved experimental 
techniques. Higher electron currents at energy widths in the $\mathrm{meV}$ range are mandatory for cases in which the product of cross-section and target density is intrinsically low. While laser photoelectron sources involving gas phase atoms are increasingly affected by energy broadening due to photoion space charge at currents above about $100 \mathrm{pA}$, photoemission from suitably prepared solid state surfaces (e.g. doped GaAs) holds promise for major progress (e.g. Pastuszka et al., 2000) when much higher electron currents at energy widths around $10 \mathrm{meV}$ are needed. This technique is, however, technologically demanding in that ultrahigh vacuum requirements for the electron source have to be combined with a gaseous target of sufficient density.

Most of the work discussed in this review dealt with molecules and clusters in their electronic and vibrational ground state. Regarding electron collisions with electronically excited atoms and molecules, the status of the field has been recently surveyed by Christophorou and Olthoff (2001b). The obvious experimental challenge lies in the preparation of a sufficient density of selectively excited states to allow electron collision studies at a decent energy resolution. Advances in laser technology (i.e. high intensity laser diodes as pumps of solid state lasers in combination with efficient nonlinear frequency conversion techniques) hold hope for the availability of broadly tunable, narrowband lasers with high repetition rate and intensity. Such laser systems could access electronically excited molecules with intermediate lifetimes comparable to the molecular transit time through the collision region with the electron beam.

For molecules in the electronic ground state, it is known that electron collision processes can depend very strongly on the initial vibrational state, yet rather few studies went beyond the conventional approach of thermally heating the target molecules in investigating the effects of vibrational excitation. However, the hope for efficient preparation of selectively excited vibrational modes has recently substantially increased. Although the STIRAP method (Vitanov et al., 2001) involving cw lasers is suitable only in few cases (as discussed for $\mathrm{Na}_{2}$ in this review), it may be applied to a broader range of molecules when pulsed lasers with sufficient coherence, pulse energy and repetition rate become available. With the advent of efficient optical parametric oscillators (OPO) and generators (OPG) in the infrared region (so far up to about $4 \mu \mathrm{m}$ ), which involve quasi-phase matching in periodically poled solid state crystals such as $\mathrm{LiNbO}_{3}$ (see, e.g., Kovalchuk et al., 1997, for a cw single mode OPO and Bäder et al., 2003, for a transform-limited, seeded nanosecond OPG with $10 \mathrm{kHz}$ repetition rate), the future looks promising for efficient excitation of many dipole-allowed vibrational transitions, including the $\mathrm{C}-\mathrm{H}$ stretch mode. This should allow a broad range of high resolution 
studies of electron scattering and attachment involving vibrationally excited molecules.

\section{Acknowledgements}

The authors are grateful to D. Field, N. Jones, and J. P. Ziesel for communicating data of their total scattering work (Figs. 14, 32, 36), to C. M. Surko for providing data on positron annihilation (Fig. 54), to M. Ćížek and J. Horáček for theoretical VE spectra for HF (Fig. 18), to T. Sommerfeld for his work on $\mathrm{CO}_{2}$ (Fig. 35), and to O. Kaufmann, $\mathrm{K}$. Bergmann and W. Meyer for files of their work on DA to sodium dimers (Figs. 27, 28). Many colleagues have contributed to the work on which substantial fractions of this review are based; in particular, we gratefully acknowledge members of the Kaiserslautern group, especially D. Klar, A. Schramm, J. M. Weber, E. Leber, and S. Barsotti, for their experimental achievements in developing and applying the LPA method. We gratefully acknowledge several colleagues for fruitful discussions, in particular T. Sommerfeld, L. S. Cederbaum, A. K. Kazansky, P. D. Burrow, G. A. Gallup, W. Meyer, M. Ćižek, J. Horáček, W. Domcke, J. P. Gauyacq, R. N. Compton, Y. N. Vasil'ev, T. M. Miller, E. Illenberger, and F. Linder. We thank G. Koschmann for secretarial support.

HH and MWR gratefully acknowledge support of the LPA work by the Deutsche Forschungsgemeinschaft through Schwerpunktprogramm Molekulare Cluster and through Forschergruppe Niederenergetische Elektronenstoßprozesse. IIF thanks the members of the Forschergruppe for their hospitality during his several stays at Fachbereich Physik of the University of Kaiserslautern. IIF has been supported by the U.S. National Science Foundation through Grant No. PHY-0098459. MA thanks the Swiss National Science Foundation for support (project 2000-067877.02).

\section{References}

Abouaf, R., and Teillet-Billy, D. (1977). Fine structure in dissociative attachment cross sections for $\mathrm{HCl}$ and DCl. J. Phys. B 10, 2261-2268.

Abouaf, R., and Teillet-Billy, D. (1980). Fine structure in the dissociative attachment cross sections for $\mathrm{HBr}$ and HF. Chem. Phys. Lett. 73, 106-109.

Ajello, J. M., and Chutjian, A. (1979). Line shapes for attachment of threshold electrons to $\mathrm{SF}_{6}$ and $\mathrm{CFCl}_{3}$ : Threshold photoelectron (TPSA) studies of $\mathrm{Xe}, \mathrm{CO}$, and $\mathrm{C}_{2} \mathrm{H}_{2}$. J. Chem. Phys. 71, 1079-1087. 
Alajajian, S. H., Bernius, M. T., and Chutjian, A. (1988). Electron attachment lineshapes, cross sections and rate constants at ultra-low energies in several halomethyl and haloethyl molecules. J. Phys. B 21, 4021-4033.

Alge, E., Adams, N. G., and Smith, D. (1984). Rate coefficients for the attachment reactions of electrons with $\mathrm{c}-\mathrm{C}_{7} \mathrm{~F}_{14}, \mathrm{CH}_{3} \mathrm{Br}, \mathrm{CF}_{3} \mathrm{Br}, \mathrm{CH}_{2} \mathrm{Br}_{2}$ and $\mathrm{CH}_{3} \mathrm{I}$ determined between 200 and $600 \mathrm{~K}$ using the FALP technique. J. Phys. B. 17, 3827-3833.

Allan, M. (1989). Study of triplet states and short-lived negative ions by means of electron impact spectroscopy. J. Electron Spectrosc. 48, 219-351.

Allan, M. (1995). Measurement of absolute differential cross sections for vibrational excitation of $\mathrm{O}_{2}$ by electron impact. J. Phys. B 28, 5163-5175.

Allan, M. (2000). Excitation of the $2^{3} \mathrm{~S}$ state of helium by electron impact from threshold to $24 \mathrm{eV}$ : measurements with the 'magnetic angle changer'. J. Phys. B 33, L215-L220.

Allan, M. (2001a). Selectivity in the excitation of fermi-coupled vibrations in $\mathrm{CO}_{2}$ by impact of slow electrons. Phys. Rev. Lett. 87, 033201 (1-4).

Allan, M. (2001b). Threshold peaks in vibrational excitation by electron impact, In: I. I. Fabrikant, G. A. Gallup, and P. D. Burrow, Eds. "Int. Symp. on Electron-Molecule Collisions and Swarms," Abstracts. pp. 67-70, Lincoln, NE, USA.

Allan, M. (2001c). Threshold peaks in vibrational excitation of polyatomic molecules. In: S. Datz, M. E. Bannister, H. F. Krause, L. H. Saddiq, D. R. Schultz, And C. R. Vane, Eds. "Abstracts of contributed papers of the 22. Int. Conf. on Photonic Electronic and Atomic Collisions (XXII ICPEAC)," pp. 275, Santa Fe, N.M., USA.

Allan, M. (2002a). Vibrational structures in electron- $\mathrm{CO}_{2}$ scattering below the ${ }^{2} \Pi_{u}$ shape resonance. J. Phys. B 35, L387-L395.

Allan, M. (2002b). Selective excitation of fermi-coupled vibrations in $\mathrm{CO}_{2}$ by impact of slow electrons. In J. Burgdörfer, J. S. Cohen, S. Datz, C. R. Vane, Eds. "Photonic, Electronic, and Atomic Collisions," pp. 284-287, Rinton Press, Princeton, NY, USA.

Allan, M., and Fabrikant, I. I. (2002). Threshold peaks and structures in vibrational excitation of $\mathrm{CH}_{3} \mathrm{I}$ by electron impact. $J$. Phys. B 35, 1025-1034.

Allan, M., and Moreira, O. (2002). Excitation of the symmetric and antisymmetric stretch vibrations of $\mathrm{H}_{2} \mathrm{O}$ by electron impact. J. Phys. B. 35, L37-L42.

Allan, M., Čižek, M., Horáček, J., and Domcke, W. (2000). Electron scattering in cooled HCl: boomerang structures and outer-well resonances in elastic and vibrational excitation cross sections. J. Phys. B 33, L209-L213.

Allan, M., Craig, N. C., and McCarty, L. V. (2002). Vibrational excitation of cis- and trans-1,2difluoroethenes by electron impact: effect of dipole moment on the threshold peaks. J. Phys. B $35,523-532$.

Allan, M. (2003). Threshold peaks and structures in elastic and vibrationally inelastic electron impact cross sections for $\mathrm{CS}_{2}$. J. Phys. B 36, 2489-2500.

Allan, M. and Skalický, T. (2003). Structures in elastic vibrational, and dissociative electron attachment cross sections in $\mathrm{N}_{2} \mathrm{O}$ near threshold. J. Phys. B. 3397-3409.

Andersen, N., and Bartschat, K. (1996). Complete experiments in electron-atom collisions. $A d v$. At. Mol. Opt. Phys. 36, 1-87.

Andersen, T., Haugen, H. K., and Hotop, H. (1999). Binding energies in atomic negative ions: III. J. Phys. Chem. Ref. Data 28, 1511-1533.

Antoni, T., Jung, K., Ehrhardt, H., and Chang, E. S. (1986). Rotational branch analysis of the excitation of the fundamental vibrational modes of $\mathrm{CO}_{2}$ by slow electron collisions. J. Phys. $B$ 19, 1377-1396.

Azria, R., Abouaf, R., and Teillet-Billy, D. (1982). Symmetry of $\mathrm{Cl}_{2}^{-*}$ resonant states formed in dissociative electron attachment reaction on $\mathrm{Cl}_{2}$. J. Phys. B 15, L569-L574. 
Bäder, U., Mattern, T., Bauer, T., Bartschke, J., Rahm, M., Borsutzky, A., and Wallenstein, R. (2003). Pulsed nanosecond optical parametric generator based on periodically poled lithium niobate. Opt. Commun. 217, 375-380.

Bailey, C. G., Lavrich, D. J., Serxner, D., and Johnson, M. A. (1996). Autodetachment from vibrational levels of the $O_{2}^{-} A^{2} \Pi_{\mathrm{u}}$ resonance across its dissociation limit by photoexcitation from $\mathrm{O}_{2}^{-} \mathrm{X}^{2} \Pi_{\mathrm{g}}$. J. Chem. Phys. 105, 1807-1814.

Bardsley, J. N. (1968). Configuration interaction in the continuum states of molecules. J. Phys. B 1, 349-364.

Bardsley, J. N., and Mandl, F. (1968). Resonant scattering of electrons by molecules. Rep. Prog. Phys. 31, 471-511.

Bardsley, J. N., and Wadehra, J. M. (1983). Dissociative attachment in $\mathrm{HCl}, \mathrm{D}_{2}$, and $\mathrm{F}_{2}$. J. Chem. Phys. 78, 7227-7234.

Barnes, L. D., Gilbert, S. J., and Surko, C. M. (2003). Energy-resolved positron annihilation for molecules. Phys. Rev. A 67, 032706 (1-11).

Barsotti, S., Leber, E., Ruf, M.-W., and Hotop, H. (2002a). High resolution study of cluster anion formation in low-energy electron collisions with molecular clusters of $\mathrm{CO}_{2}, \mathrm{CS}_{2}$, and $\mathrm{O}_{2}$. Int. J. Mass Spectrom. 220, 313-330.

Barsotti, S., Ruf, M.-W., and Hotop, H. (2002b). Clear experimental evidence for p-wave attachment-threshold behavior in electron attachment to chlorine molecules. Phys. Rev. Lett. 89, $083201(1-4)$.

Barsotti, S., Ruf, M.-W., and Hotop, H. (2003a). Laser photoelectron attachment to $\mathrm{SF}_{6}$ molecules in a seeded supersonic beam at temperatures from $300 \mathrm{~K}$ to $600 \mathrm{~K}$. (in preparation).

Barsotti, S., Sommerfeld, T., Ruf, M.-W., and Hotop, H. (2003b). High resolution study of cluster anion formation in low-energy electron collisions with OCS clusters. Int. J. Mass. Spectrom. (submitted).

Baz', A. I. (1958). The energy dependence of a scattering cross section near the threshold of a reaction. Sov. Phys. JETP 6, 709-713.

Baz', A. I. (1961). Energy dependence of cross sections near the "threshold" for unstable particle production. Sov. Phys. JETP 13, 1058-1061.

Becker, K. H., McCurdy, C. W., Orlando, T. W., and Rescigno, T. M. (Eds.) (2000). "Current Status and Future Perspectives of Electron Interactions with Molecules, Clusters, Surfaces, and Interfaces," Stevens Inst. Technol., Hoboken, NJ, USA.

Bernath, M., Dragún, O., Spinella, M. R., Massmann, H., and Pacheco, J. M. (1995). Response of metal clusters to elastic electron impact. Phys. Rev. A 52, 2173-2178.

Bethe, H. A. (1935). Theory of disintegration of nuclei by neutrons. Phys. Rev. 47, 747-759.

Beyer, T., Nestmann, B. M., Peyerimhoff, S. D. (2001). Resonant features of inelastic electron scattering off $\mathrm{CF}_{3} \mathrm{Cl}$ in the low-energy region. J. Phys. B 34, 3703-3716.

Birtwistle, D. T., and Herzenberg, A. (1971). Vibrational excitation of $\mathrm{N}_{2}$ by resonance scattering of electrons. J. Phys. B 4, 53-70.

Blatt, J. M., and Weisskopf, V. F. (1952). "Theoretical Nuclear Physics," Wiley, NY, USA.

Bloch, F., and Bradbury, N. E. (1935). On the Mechanism of Unimolecular Electron Capture. Phys. Rev. 48, 689-695.

Bömmels, J., Leber, E., Gopalan, A., Weber, J. M., Barsotti, S., Ruf, M.-W., and Hotop, H. (2001). Energy broadening due to photoion space charge in a high resolution laser photoelectron source. Rev. Scient. Instrum. 72, 4098-4105.

Boness, M. J. W., and Schulz, G. J. (1974). Vibrational excitation in $\mathrm{CO}_{2}$ via the $3.8 \mathrm{eV}$ resonance. Phys. Rev. A 9, 1969-1979.

Bonin, K., and Kresin, V. (1997). "Electric-Dipole Polarizabilities of Atoms, Molecules, and Clusters," World Scientific, Singapore. 
Boudaïfa, B., Cloutier, P., Hunting, D., Huels, M. A., and Sanche, L. (2000). Resonant formation of DNA strand breaks by low-energy $(3-20 \mathrm{eV})$ electrons. Science 287, $1658-1660$.

Brems, V., Beyer, T., Nestmann, B. M., Meyer, H.-D., and Cederbaum, L. S. (2002). Ab initio study of the resonant electron attachment top the $\mathrm{F}_{2}$ molecule. J. Chem. Phys. 117, 10635-10647.

Brunger, M. J., and Buckman, S. J. (2002). Electron-molecule scattering cross-sections. I. Experimental techniques and data for diatomic molecules. Phys. Rep. 357, 215-458.

Brüning, F., Matejcik, S., Illenberger, E., Chu, Y., Senn, G., Muigg, D., Denifl, G., and Märk, T. D. (1998). Effects of temperature on the dissociative electron attachment to $\mathrm{N}_{2} \mathrm{O}$. Chem. Phys. Lett. 292, 177-182.

Buck, U., and Meyer, D. (1986). Electron bombardment fragmentation of Ar van der Waals clusters by scattering analysis. J. Chem. Phys. 84, 4854-4861.

Buck, U. (1988). Properties of neutral clusters from scattering experiments. J. Phys. Chem. 92, 1023-1031.

Buckman, S. J., and Clark, C. W. (1994). Atomic negative-ion resonances. Rev. Mod. Phys. 66 , 539-655.

Burke, P. G. (1979). Theory of low energy electron-molecule collisions. Adv. At. Mol. Phys. 15, 471-506.

Burrow, P. D. (1974). Threshold structure in the elastic cross section for scattering of slow electrons from $\mathrm{HCl}$ and $\mathrm{HBr}$. J. Phys. B 7, L385-L388.

Burrow, P. D., Modelli, A., Chiu, N. S., and Jordan, K. D. (1982). Temporary negative ions in the chloromethanes $\mathrm{CHCl}_{2} \mathrm{~F}$ and $\mathrm{CCl}_{2} \mathrm{~F}_{2}$ : Characterization of the $\sigma^{*}$ orbitals. J. Chem. Phys. 77, 2699-2701.

Burrow, P. D., Gallup, G. A., Fabrikant, I. I., and Jordan, K. D. (1997). Dissociative attachment studies of halogen-containing molecules: Problems, applications and challenges. Aust. J. Phys. 49, 403-423.

Carman, H. S. Jr., Klots, C. E., and Compton, R. N. (1990). Isotope-dependent rate constants for $\mathrm{CS}_{2}^{-}$formation in $\mathrm{Cs}(n s, n d)+\mathrm{CS}_{2}$ collisions. J. Chem. Phys. 92, 5751-5752.

Chantry, P. J. (1969). Temperature dependence of dissociative attachment in $\mathrm{N}_{2} \mathrm{O}$. J. Chem. Phys. 51, 3369-3379.

Chantry, P. J. (1982). Negative ion formation in gas lasers. In E. W. McDaniel and W. L. Nighan, Eds. "Gas Lasers. Applied Atomic Collision Physics 3," pp. 35-70, Academic Press, New York.

Chase, D. M. (1956). Adiabatic approximation for scattering processes. Phys. Rev. 104, 838-842.

Chen, C. L., and Chantry, P. J. (1979). Photon-enhanced dissociative electron attachment in $\mathrm{SF}_{6}$ and its isotopic selectivity. J. Chem. Phys. 71, 3897-3907.

Chen, E. C. M., and Wentworth, W. R. (1983). Determination of molecular electron affinities using the electron capture detector in the pulse sampling mode at steady state. J. Phys. Chem. 87, 45-49.

Christophorou, L. G. (1976). Electron attachment to molecules in dense gases ("quasi-liquids"). Chem. Rev. 76, 409-423.

Christophorou, L. G. (1978). The lifetimes of metastable negative ions. Adv. in Electronics and Electron Physics 46, 55-129.

Christophorou, L. G. (Ed.) (1984). "Electron-Molecule Interactions and their Applications," Vols. 1 and 2, Academic Press, New York.

Christophorou, L. G. (1987). Electron attachment and detachment processes in electronegative gases. Contrib. Plasma Phys. 27 (4), 237-281. 
Christophorou, L. G. (1996). The dependence of the thermal electron attachment rate constant in gases and liquids on the energy position of the electron attaching state. Z. Phys. Chem. 195, 195-215.

Christophorou, L. G., and Olthoff, J. K. (1999). Electron interactions with $\mathrm{Cl}_{2}$. J. Phys. Chem. Ref. Data 28, 131-169.

Christophorou, L. G., and Olthoff, J. K. (2000). Electron interactions with SF 6 . J. Phys. Chem. Ref. Data 29, 267-330.

Christophorou, L. G., and Olthoff, J. K. (2001a). Electron collision data for plasma-processing gases. Adv. At. Mol. Opt. Phys. 44, 59-98.

Christophorou, L. G., and Olthoff, J. K. (2001b). Electron Interactions with Excited Atoms and Molecules. Adv. At. Mol. Opt. Phys. 44, 155-293.

Chu, S. C., and Burrow, P. D. (1990). Dissociative attachment of electrons in the chloromethanes. Chem. Phys. Lett. 172, 17-22.

Chutjian, A., and Alajajian, S. H. (1985). $s$-wave threshold in electron attachment: Observations and cross sections in $\mathrm{CCl}_{4}$ and $\mathrm{SF}_{6}$ at ultralow electron energies. Phys. Rev. A 31, 2885-2892.

Chutjian, A., and Alajajian, S. H. (1987). Electron attachment in $\mathrm{F}_{2}$ : Conclusive demonstration of nonresonant, s-wave coupling in the limit of zero electron energy. Phys. Rev. A $\mathbf{3 5}$, 4512-4514.

Chutjian, A. (1992). Electron attachment to molecules at ultralow electron energies. In: W. R. MacGillivray, I. E. McCarthy, M. C. Standage, Eds. "Electronic and Atomic Collisions," pp. 127-138, Adam Hilger, Bristol.

Chutjian, A., Garscadden, A., and Wadehra, J. M. (1996). Electron attachment to molecules at low electron energies. Phys. Rep. 264, 393-470.

Č́žžek, M., Horáček, J., and Domcke, W. (1999). Associative detachment, dissociative attachment, and vibrational excitation of $\mathrm{HCl}$ by low-energy electrons. Phys. Rev. A 60 , 2873-2881.

Č́žzek, M., Horáček, J., Sergenton, A.-Ch., Popovic, D. B., Allan, M., Domcke, W., Leininger, T., and Gadea, F. X. (2001a). Inelastic low-energy electron collisions with the $\mathrm{HBr}$ and $\mathrm{DBr}$ molecules: Experiment and theory. Phys. Rev. A 63, 062710(1-14).

Č́žžek, M., Horáček, J., Thiel, F. A. U., and Hotop, H. (2001b). Associative detachment in low-energy collisions between hydrogen atoms and atomic halogen anions. J. Phys. B 34, 983-1004.

Č́žžek, M., Horáček, J., Allan, M., Fabrikant, I. I., Domeke, W. (2003). Vibrational excitation of hydrogen fluoride by low-energy electrons: theory and experiment. J. Phys. B 36, 2837-2849.

Clark, C. W. (1979). Electron scattering from diatomic polar molecules. II. Treatment by frame transformations. Phys. Rev. A 20, 1875-1889.

Compton, R. N. (1980). Electron attachment to molecules. In: N. Oda and, K. Takayanagi, Eds. "Electronic and Atomic Collisions," pp. 251-262, North-Holland, Amsterdam.

Compton, R. N., and Hammer, N. I. (2001). Multipole-bound molecular anions. Adv. in Gas-Phase Ion Chemistry, Vol. 4, 257-305. Elsevier Science B.V.

Compton, R. N., Reinhardt, P. W., and Cooper, C. D. (1975). Collisional ionisation of $\mathrm{Na}, \mathrm{K}$, and $\mathrm{Cs}$ by $\mathrm{CO}_{2}, \mathrm{COS}$, and $\mathrm{CS}_{2}$ : molecular electron affinities. J. Chem. Phys. 63, 3821-3827.

Compton, R. N., Carman, H. S. Jr., Desfrançois, C., Abdoul-Carmine, H., Schermann, J.-P., Hendricks, J. H., Lyapustina, S. A., and Bowen, K. H. (1996). On the binding of electrons to nitromethane: dipole and valence bound anions. J. Chem. Phys. 105, 3472-3478. 
Connerade, J.-P., Gerchikov, L. G., Ipatov, A. N., and Senturk, Ş. (2000). Resonance effects in inelastic scattering of low-energy electrons from metallic clusters. J. Phys. B 33, 5109-5120.

Crawford, O. H., and Garrett, W. R. (1977). Electron affinities of polar molecules. J. Chem. Phys. 66, 4968-4970.

Crawford, O. H., and Koch, B. J. D. (1974). Diabatic capture in dissociative attachment. J. Chem. Phys. 60, 4512-4519.

Crompton, R. W. (1994). Benchmark measurements of cross sections for electron collisions: Electron swarm methods. Adv. At. Mol. Opt. Phys. 33, 97-148.

Cubric, Mercer, D. J. L., Channing, J. M., King, G. C., and Read, F. H. (1999) A study of inelastic electron scattering in He covering the complete angular range from $0^{\circ}$ to $180^{\circ}$. J. Phys. B 32, L45-L50.

Cvejanovic, S. (1993). Threshold and resonant features in the vibrational excitation functions of hydrogen halides. In: T. Andersen, B. Fastrup, F. Folkmann, H. Knudsen, Eds. "The Physics of Electronic and Atomic Collisions," AIP Conf. Proc. Vol. 295, pp. 390-401, A.I.P., New York.

Damburg, R. J. (1968). Threshold law for model with long-range interaction. J. Phys. B 1, 1001-1003.

Datskos, P. G., Christophorou, L. G., and Carter, J. G. (1990). Temperature-enhanced electron attachment to $\mathrm{CH}_{3} \mathrm{Cl}$. Chem. Phys. Lett. 168, 324-329.

Datskos, P. G., Christophorou, L. G., and Carter, J. G. (1992). Effect of temperature on the attachment of slow $(\leq 1 \mathrm{eV})$ electrons to $\mathrm{CH}_{3} \mathrm{Br}$. J. Chem. Phys. 97, 9031-9035.

Desfrançois, C., Khelifa, N., Schermann, J. P. (1989). Very low energy electron attachment to $\mathrm{SF}_{6}$ clusters. J. Chem. Phys. 91, 5853-5854.

Desfrançois, C., Khelifa, N., Schermann, J.-P., Kraft, T., Ruf, M.-W., and Hotop, H. (1993). Energy exchanges following very low energy electron attachment to neat $\mathrm{CS}_{2}$ and $\mathrm{CS}_{2}$ containing clusters. Z. Phys. D 27, 365-369.

Desfrançois, C., Abdoul-Carime, H., Adjouri, C., Khelifa, N., Schermann, J. P. (1994a). Dipole binding to a strongly polar molecule and its homogeneous clusters: Magic distribution of Acetonitrile cluster anions. Europhys. Lett. 26, 25-30.

Desfrançois, C., Baillon, B., Schermann, J.-P., Arnold, S. T., Hendricks, J. H., and Bowen, K. H. (1994b). Prediction and observation of a new, ground state, dipole-bound dimer anion: the mixed water/ammonia system. Phys. Rev. Lett. 72, 48-51.

Desfrançois, C., Abdoul-Carime, H., Khelifa, N., and Schermann, J.-P. (1994c). From 1/r to $1 / r^{2}$ potentials: electron exchange between Rydberg atoms and polar molecules. Phys. Rev. Lett. 73, 2436-2439.

Desfrançois, C., Abdoul-Carime, H., and Schermann, J.-P. (1996). Ground-state dipole-bound anions. Int. J. Mod. Phys. B 10, 1339-1395.

Dessent, C. E. H., Bailey, C. G., and Johnson, M. A. (1996). J. Chem. Phys. 105, 10416-10423.

Domcke, W. (1991). Theory of resonance and threshold effects in electron-molecule collisions: the projection-operator approach. Phys. Rep. 208, 97-188.

Domcke, W., and Cederbaum, L. S. (1977). Theory of the vibrational structure of resonances in electron-molecule scattering. Phys. Rev. A 16, 1465-1482.

Domcke, W., and Cederbaum, L. S. (1981). On the interpretation of low-energy electron-HCl scattering phenomena. J. Phys. B 14, 149-173.

Domcke, W., and Mündel, C. (1985). Calculation of cross sections for vibrational excitation and dissociative attachment in $\mathrm{HCl}$ and $\mathrm{DCl}$ beyond the local-complex-potential approximation. J. Phys. B 18, 4491-4509.

Dubé, L., and Herzenberg, A. (1977). Vibrational excitation of polar molecules by slow electrons: HCl. Phys. Rev. Lett. 38, 820-823. 
Dubé, L., and Herzenberg, A. (1979). Absolute cross sections from the "boomerang model" for resonant electron-molecule scattering. Phys. Rev. A 20, 194-213.

Dunning, F. B. (1995). Electron-molecule collisions at very low electron energies. J. Phys. B 28, $1645-1672$.

Dzuba, V. A., Flambaum, V. V., Gribakin, G. F., and King, W. A. (1995). Bound states of positrons and neutral atoms. Phys. Rev. A 52, 4541-4546.

Elhamidi, O., Pommier, J., and Abouaf, R. (1997). Low-energy attachment to fullerenes $\mathrm{C}_{60}$ and $\mathrm{C}_{70}$ in the gas phase. J. Phys. B 30, 4633-4642.

Engelking, P. C. (1982). Strong electron-dipole coupling in photodetachment of molecular negative ions: Anomalous rotational thresholds. Phys. Rev. A 26, 740-745.

Engelking, P. C., and Herrick, D. R. (1984). Effects of rotational doubling on the anomalous photodetachment thresholds resulting from electron-dipole interaction. Phys. Rev. A 29, 2425-2428.

Estrada, H., and Domcke, W. (1985). On the virtual-state effect in low-energy electron- $\mathrm{CO}_{2}$ scattering. J. Phys. B 18, 4469-4479.

Etters, R. D., Flurchick, K., Pan, R. P., Chandrasekharan, V. (1981). Thermodynamic properties and phase transitions in $\mathrm{CO}_{2}$ molecular clusters. J. Chem. Phys. 75, 929-936.

Fabrikant, I. I. (1977). Threshold behavior of the cross sections for scattering of electrons by polar molecules. Sov. Phys. JETP 46, 693-697.

Fabrikant, I. I. (1978). The influence of long-range interaction on the vibrational excitation of polar molecules by electrons. J. Phys. B 11, 3621-3633.

Fabrikant, I. I. (1979). The influence of $\mathrm{a}^{-\mathrm{s}}$ potential on electron scattering by polar molecules. J. Phys. B 12, 3599-3610.

Fabrikant, I. I. (1983). Frame transformation effective-range theory: application to interaction of polar molecules with electrons. J. Phys. B 16, 1269-1282.

Fabrikant, I. I. (1984). Effective-range analysis of low-energy electron scattering by non-polar molecules. J. Phys. B 17, 4223-4233.

Fabrikant, I. I. (1985). $R$-matrix theory of vibrational excitation of the $\mathrm{HCl}$ molecule by slow electrons in the adiabatic approximation. J. Phys. B 18, 1873-1879.

Fabrikant, I. I. (1986). $R$-matrix theory of inelastic processes in low-energy electron collisions with $\mathrm{HCl}$ molecule. Z. Physik 3, 401-410.

Fabrikant, I. I. (1990). Resonance processes in e- $\mathrm{HCl}$ collisions: comparison of the $R$-Matrix and the nonlocal-complex-potential methods. Comments At. Mol. Phys. 24, 37-52.

Fabrikant, I. I. (1991a). Quasiclassical $R$-matrix theory of inelastic processes in collisions of electrons with $\mathrm{HCl}$ molecules. Phys. Rev. A 43, 3478-3486.

Fabrikant, I. I. (1991b). A model describing inelastic processes in low-energy electron collisions with methyl chloride. J. Phys. B 24, 2213-2225.

Fabrikant, I. I., Kalin, S. A., and Kazansky, A. K. (1991). Inelastic processes in e- $\mathrm{HCl}$ collisions in the energy range including the dissociation threshold. J. Chem. Phys. 95, 4966-4971.

Fabrikant, I. I., Kalin, S. A., and Kazansky, A. K. (1992). Resonant $R$-matrix theory of inelastic processes in collisions of electrons with HF molecules. J. Phys. B 25, 2885-2898.

Fabrikant, I. I. (1994). Semiempirical calculations of inelastic electron-methylchloride scattering. J. Phys. B 27, 4325-4336.

Fabrikant, I. I. (1996). Electron scattering by neutral targets at milli- and submilli-electron-volt energies. Comments At. Mol. Phys. 32, 267-279.

Fabrikant, I. I., and Hotop, H. (2001). Low-energy behavior of exothermic dissociative electron attachment. Phys. Rev. A 63, 022706(1-10).

Fabrikant, I. I., and Wilde, R. S. (1999). Evidence of virtual dipole-supported states in electron scattering by methyl chloride. J. Phys. B 32, 235-240. 
Fabrikant, I. I., Leininger, T., and Gadéa, F. X. (2000). Low-energy dissociative electron attachment to $\mathrm{Cl}_{2}$ molecules. J. Phys. B 33, 4575-4580.

Feketeova, L., Skalny, D. J., Hanel, G., Gstir, B., Francis, M., and Märk, T. D. (2003). Production of $\mathrm{Cl}^{-}$via dissociative electron attachment to $\mathrm{Cl}_{2}$. Int. J. Mass Spectrom. 223-224, 661-667.

Ferch, J., Raith, W., Schröder, K. (1982). Total cross section measurement for e- $\mathrm{SF}_{6}$ scattering down to $0.036 \mathrm{eV}$. J. Phys. B 15, L175-L178.

Fermi, E. (1934). Sopra lo spostamento per pressione delle righe elevate delle serie spettrali. Nuovo Cimento 11, 157-166.

Fermi, E., and Teller, E. (1947). The capture of negative mesotrons in matter. Phys. Rev. 72 , 399-408.

Field, D., Mrotzek, G., Knight, D. W., Lunt, S. L., and Ziesel, J.-P. (1988). High-resolution studies of electron scattering by molecular oxygen. J. Phys. B 21, 171-188.

Field, D., Knight, D. W., Mrotzek, G., Randell, J., Lunt, S. L., Ozenne, J. B., and Ziesel, J. P. (1991a). A high-resolution synchrotron photoionization spectrometer for the study of low-energy electron-molecule scattering. Meas. Sci. Technol. 2, 757-769.

Field, D., Lunt, S. L., Mrotzek, G., Randell, J., and Ziesel, J. P. (1991b). High resolution electron scattering from carbon dioxide. J. Phys. B 24, 3497-3506.

Field, D., Jones, N. C., Gingell, J. M., Mason, N. J., Lunt, S. L., and Ziesel, J.-P. (2000). Electron scattering in chlorine dioxide. J. Phys. B. 33, 1039-1046.

Field, D., Lunt, S. L., and Ziesel, J.-P. (2001a). The quantum world of cold electron collisions. Acc. Chem. Res. 34, 291-296.

Field, D., Jones, N. C., Lunt, S. L., and Ziesel, J.-P. (2001b). Experimental evidence for a virtual state in a cold collision: electrons and carbon dioxide. Phys. Rev. A 64, 022708 (1-6).

Field, D., Jones, N. C., Lunt, S. L., Ziesel, J.-L., and Gulley, R. G. (2001c). Low energy electron scattering in $\mathrm{CF}_{2} \mathrm{Cl}_{2}$ and $\mathrm{CF}_{3} \mathrm{Cl}$. J. Chem. Phys. 115, 3045-3052.

Filippelli, A. R., Lin, C. C., Anderson, L. W., and McConkey, J. W. (1994). Principles and methods for measurement of electron impact excitation cross sections for atoms and molecules by optical techniques. Adv. At. Mol. Opt. Phys. 33, 1-62.

Finch, C. D., Popple, R. A., Nordlander, P., and Dunning, F. B. (1995). Formation of longlived $C_{60}^{-}$ions in Rydberg atom- $\mathrm{C}_{60}$ collisions. Chem. Phys. Lett. 244, 345-349.

Fiquet-Fayard, F. (1974). Theoretical investigation of dissociative attachment in $\mathrm{HCl}$ and $\mathrm{DCl}$. J. Phys. B 7, 810-816.

Frey, M. T., Hill, S. B., Ling, X., Smith, K. A., Dunning, F. B., and Fabrikant, I. I. (1994). Inelastic electron-dipole-molecule scattering at sub-milli-electron-volt energies: Possible role of dipole-supported states. Phys. Rev. A 50, 3124-3128.

Frey, M. T., Hill, S. B., Smith, K. A., Dunning, F. B., and Fabrikant, I. I. (1995). Studies of electron-molecule scattering at microelectronvolt energies using very-high- $n$ Rydberg atoms. Phys. Rev. Lett. 75, 810-813.

Frey, M. T., Ling, X., Lindsay, B. G., Smith, K. A., and Dunning, F. B. (1993). Use of the Stark effect to minimize residual electric fields in an experimental volume. Rev. Sci. Instrum. 64 (12), 3649-3650.

Gailitis, M. (1970). Influence of close coupling on the threshold behavior of the cross sections for the excitation of atoms by electrons. Theor. Math. Phys. 3, 572-581.

Gailitis, M., and Damburg, R. (1963). The influence of close coupling on the threshold behaviour of cross sections of electron-hydrogen scattering. Proc. Phys. Soc. 82, 192-200.

Gallup, G. A., Xu, Y., and Fabrikant, I. I. (1998). Nonlocal theory of dissociative electron attachment to $\mathrm{H}_{2}$ and HF molecules. Phys. Rev. A 57, 2596-2607. 
Gallup, G. A., Aflatooni, K., and Burrow, P. D. (2003). Dissociative electron attachment near threshold, thermal attachment rates, and vertical attachment energies of chloroalkanes. J. Chem. Phys. 118, 2562-2574.

Gauthier, M. (1988). Spectra and structure of nitrous oxide dimers and clusters. J. Chem. Phys. 88, 5439-5449.

Gauyacq, J. P. (1982). Associative detachment in collisions between negative halogen ions and hydrogen atoms. J. Phys. B 15, 2721-2739.

Gauyacq, J. P. (1983). Associative detachment and vibrational excitation in the $\mathrm{e}^{-}$-HF system. J. Phys. B 16, 4049-4058.

Gauyacq, J.-P. (1987). "Dynamics of Negative Ions," World Scientific, Singapore.

Gauyacq, J. P., and Herzenberg, A. (1982). Nuclear-excited Feshbach resonances in e+HCl scattering. Phys. Rev. A 25, 2959-2967.

Gauyacq, J. P., and Herzenberg, A. (1984). The attachment of very slow electrons to polyatomic molecules. J. Phys. B 17, 1155-1171.

Gerchikov, L. G., Ipatov, A. N., and Solov'yov, A. V., and Greiner, W. (1998). Excitation of multipole plasmon resonances in clusters by electron impact. J. Phys. B 31, 3065-3078.

Gianturco, F. A., and Lucchese, R. R. (2001). Electron scattering from gaseous $\mathrm{SF}_{6}$ : comparing calculations with experiments. J. Chem. Phys. 114, 3429-3439.

Gianturco, F. A., Lucchese, R. R., and Sanna, N. (1999). Computed elastic cross sections and angular distributions of low-energy electron scattering from gas phase $\mathrm{C}_{60}$ fullerene. J. Phys. B 32, 2181-2193.

Gilbert, S. J., Barnes, L. D., Sullivan, J. P., and Surko, C. M. (2002). Vibrational-resonance enhancement of positron annihilation in molecules. Phys. Rev. Lett. 88, 043201(1-4).

Gopalan, A., Bömmels, J., Götte, S., Landwehr, A., Franz, K., Ruf, M.-W., Hotop, H., and Bartschat, K. (2003). A novel electron scattering apparatus combining a laser photoelectron source and a triply differentially pumped supersonic beam target: characterization and results for the $\mathrm{He}^{-}\left(1 \mathrm{~s} 2 \mathrm{~s}^{2}\right)$ resonance. Eur. Phys. J. D 22, 17-29.

Götte, S., Gopalan, A., Bömmels, J., Ruf, M.-W., and Hotop, H. (2000). A triply differentially pumped supersonic beam target for high-resolution collision studies. Rev. Scient. Instrum. 71, 4070-4077.

Gribakin, G. F. (2000). Mechanisms of positron annihilation on molecules. Phys. Rev. A 61, $022720(1-13)$.

Gribakin, G. F. (2001). Theory of positron annihilation on molecules. In: F. A. Gianturco and C. M. Surko, Eds. "New Directions in Antimatter Chemistry and Physics," pp. 413-435, Kluwer Academic.

Gribakin, G. F., and Ludlow, J. (2002). Enhancement of Positron-Atom Annihilation near the positronium formation threshold. Phys. Rev. Lett. 88, 163202(1-4).

Gulley, R. J., Lunt, S. L., Ziesel, J.-P., and Field D. (1998a). Very low energy electron scattering from benzene and deuterated benzenes. J. Phys. B. 31, 2735-2751.

Gulley, R. J., Field, T. A., Steer, W. A., Mason, N. J., Lunt, S. L., Ziesel, J.-P., and Field, D. (1998b). Very low energy electron collisions with molecular chlorine. J. Phys. B. 31, 2971-2980.

Gutsev, G. L., Bartlett, R. J., and Compton, R. N. (1998). Electron affinities of $\mathrm{CO}_{2}$, OCS, and $\mathrm{CS}_{2}$. J. Chem. Phys. 108, 6756-6762.

Hahndorf, I., and Illlenberger, E. (1997). Temperature dependence of electron attachment processes. Int. J. Mass Spectrom. Ion Proc. 167/168, 87-101.

Hahndorf, I., Illenberger, E., Lehr, L., and Manz, J. (1994). Temperature effects of dissociative electron attachment to $\mathrm{CF}_{3} \mathrm{Cl}$. Chem. Phys. Lett. 231, 460-466.

Harth, K., Ruf, M.-W., and Hotop, H. (1989). Electron transfer from laser excited Rydberg 
atoms to molecules. Absolute rate constants at low and intermediate principal quantum numbers. Z. Phys. D 14, 149-165.

Hashemi, R., and Illenberger, E. (1991). Violation of the $\sigma^{-}$selection rule in electron attachment to $\mathrm{O}_{2}$ clusters. Chem. Phys. Lett. 187, 623-627.

Hashemi, R., Kühn, A., and Illenberger, E. (1990). Electron capture induced processes in molecules and molecular aggregates. Int. J. Mass Spectrom. Ion Proc. 100, 753-784.

Hashemi, R., Jaffke, T., Christophorou, L. G., and Illenberger, E. (1992). Role of inelastic electron scattering by $\mathrm{N}_{2}$ in the formation of $\left(\mathrm{O}_{2}\right)_{\mathrm{n}}^{-}$anions in mixed $\mathrm{O}_{2} / \mathrm{N}_{2}$ clusters. J. Phys. Chem. 96, 10605-10607.

Hatano, Y. (1997). Electron attachment and recombination in dense molecular media. Aust. J. Phys. 50, 615-625.

Hatano, Y., and Shimamori, H. (1981). Electron attachment in dense gases. In: L. G. Christophorou, Ed. "Electron and Ion Swarms," p. 103-116, Pergamon, New York.

Hazi, A. U., Orel, A. E., and Rescigno, T. N. (1981). Ab initio study of dissociative attachment of low-energy electrons to $\mathrm{F}_{2}$. Phys. Rev. Lett. 46, 918-922.

Herrick, D. R. (1975). Resonance-channel quantum numbers in electron-hydrogen and protonhydrogen scattering from group theory of the long-range dipole interaction. Phys. Rev. A 12, 413-424.

Herrick, D. R., and Engelking, P. C. (1984). Dipole coupling channels for molecular anions. Phys. Rev. A 29, 2421-2424.

Herzberg, G. (1945). Infrared and Raman spectra of polyatomic molecules. Molecular Spectra and Molecular Structure II. Van Nostrand Reinhold, N. Y.

Herzenberg, A. (1968). Oscillatory energy dependence of resonant electron-molecule scattering. J. Phys. B 1, 548-558.

Herzenberg, A. (1984). Vibrational excitation of molecules by slow electrons. In: I. Shimamura and K. Takayanagi, Eds. "Electron-Molecule Collisions," pp. 191-274, Plenum Press, New York.

Higgins, K., Gillan, C. J., Burke, P. G., and Noble, C. J. (1995). Low-energy electron scattering by oxygen molecules: II. Vibrational excitation. J. Phys. B 28, 3391-3402.

Hill, S. B., Frey, M. T., Dunning, F. B., and Fabrikant, I. I. (1996). Electron-hydrogen fluoride scattering at ultralow electron energies: possible role of dipole-supported states. Phys. Rev. A 53, 3348-3357.

Hoffmann, S. V., Lunt, S. L., Jones, N. C., Field, D., and Ziesel, J.-P. (2002). An undulatorbased spherical grating monochromator beamline for low energy electron-molecule scattering experiments. Rev. Sci. Instrum. 73, 4157-4163.

Horáček, J., and Domcke, W. (1996). Calculation of cross sections for vibrational excitation and dissociative attachment in electron collisions with $\mathrm{HBr}$ and $\mathrm{DBr}$. Phys. Rev. A 53, 2262-2271.

Horáček, J., Domcke, W., and Nakamura, H. (1997). Electron attachment and vibrational excitation in hydrogen iodide: calculations based on the nonlocal resonance model. Z. Phys. D 42, 181-185.

Horáček, J., Čižek, M., and Domcke, W. (1998). Generalization of the nonlocal resonance model for low-energy electron collisions with hydrogen halides: the variable threshold exponent. Theor. Chem. Acc. 100, 31-35.

Hotop, H. (2001). Dynamics of low energy electron collisions with molecules and clusters. In: L. G. Christophorou and J. K. Olthoff, Eds. "Gaseous Dielectrics IX," pp. 3-14, Kluwer Academic/Plenum Publ., New York. 
Hotop, H., Klar, D., Kreil, J., Ruf, M.-W., Schramm, A., and Weber, J. M. (1995). Studies of low energy electron collisions at sub-meV resolution. In: L. J. Dube, J. B. A. Mitchell, J. W. McConkey, and C. E. Brion, Eds. "The Physics of Electronic and Atomic Collisions," AIP Conf. Proc. Vol. 360, pp. 267-278. AIP Press, Woodbury, New York.

Howe, P.-T., Kortyna, A., Darrach, M., and Chutjian, A. (2001). Low-energy electron attachment to $\mathrm{SF}_{6}$ at sub-meV resolution using a tunable laser photoelectron method. Phys. Rev. A 64, 042706 (1-8).

Huang, J., Carman, H. S. Jr., and Compton, R. N. (1995). Low-energy electron attachment to $\mathrm{C}_{60}$. J. Phys. Chem. 99, 1719-1726.

Huo, W. H., and Gianturco, F. A. (Eds.) (1995). "Computational Methods for ElectronMolecule Collisions," Plenum, New York.

Ibach, H. (1991). "Electron Energy Loss Spectrometers," Springer Ser. Opt. Sciences, Vol. 63, Springer, Berlin, Heidelberg.

Ibach, H. (1993). Electron energy loss spectroscopy with resolution below $1 \mathrm{meV}$. J. Electron Spectrosc. 64/65, 819-823.

Iga, I., Rao, M. V. V. S., Srivastava, S. K., and Nogueira, J. C. (1996). Cross sections for the formation of $\mathrm{S}^{-}$ions by electron impact on OCS. Int. J. Mass Spectrom. Ion Proc. 155, 99-105.

Igarashi, A., Kimura, M., and Shimamura, I. (2002). Unified treatment of positron annihilation and positronium formation. Phys. Rev. Lett. 89, 123201(1-4).

Illenberger, E. (1992). Electron-attachment reactions in molecular clusters. Chem. Rev. 92, $1589-1609$.

Illenberger, E. (2000). Electron capture processes by free and bound molecules. In: C.-Y. Ng (Ed.) "Photoionization and Photodetachment (Part II)," Adv. Series in Physical Chemistry, Vol. 10 B, pp. 1063-1160, World Scientific, Singapore.

Ingólfsson, O., Weik, F., Illenberger, E. (1996). The reactivity of slow electrons with molecules at different degrees of aggregation: gas phase, clusters and condensed phase. Int. J. Mass Spectrom. Ion Processes 155, 1-68.

Ipatov, A. N., Ivanov, V. K., Agap'ev, B. D., and Ekardt, W. (1998a). Exchange and polarization effects in elastic electron scattering by metallic clusters. J. Phys. B 31, 925-934.

Ipatov, A., Connerade, J.-P., Gerchikov, L. G., and Solov'yov, A. V. (1998b). Electron attachment to metallic clusters. J. Phys. B 31, L27-L34.

Iwata, K., Greaves, R. G., Murphy, T. J., Tinkle, M. D., and Surko, C. M. (1995). Measurements of positron-annihilation rates on molecules. Phys. Rev. A 51, 473-487.

Iwata, K., Gribakin, G. F., Greaves, R. G., Kurz, C., and Surko, C. M. (2000). Positron annihilation on large molecules. Phys. Rev. A 61, 022719(1-17).

Jaffke, T., Hashemi, R., Christophorou, L. G., Illenberger, E. (1992). Mechanisms of anion formation in $\mathrm{O}_{2}, \mathrm{O}_{2} / \mathrm{Ar}$ and $\mathrm{O}_{2} / \mathrm{Ne}$ clusters; the role of inelastic electron scattering. Z. Phys. D 25, 77-85.

Jaffke, T., Illenberger, E., Lezius, M., Matejcik, S., Smith, D., and Märk, T. D. (1994). Formation of $C_{60}^{-}$and $C_{70}^{-}$by free electron capture. Activation energy and effect of the internal energy on lifetime. Chem. Phys. Lett. 226, 213-218.

Johnstone, W. M., Akther, P., and Newell, W. R. (1995). Resonant vibrational excitation of carbon dioxide. J. Phys. B 28, 743-753.

Jones, N. C., Field, D., Ziesel, J.-P., and Field, T. A. (2002). Giant resonances in cold electron scattering by $\mathrm{CS}_{2}$. Phys. Rev. Lett. 89, 093201(1-4).

Jordan, K. D., and Burrow, P.D. (1978). Studies of temporary anion states of unsaturated hydrocarbons by electron transmission spectroscopy. Acc. Chem. Res. 11(9), $341-348$. 
Kalamarides, A., Walter, C. W., Smith, K. A., and Dunning, F. B. (1988). Negative ion formation in $\mathrm{K}(n d)-\mathrm{CS}_{2}$ collisions: detection of electric-field-induced detachment from $\mathrm{CS}_{2}^{-}$. J. Chem. Phys. 89, 7226-7228.

Kalin, S. A., and Kazansky, A. K. (1990). The semiclassical version of the non-local resonance theory of electron-molecule collisions. J. Phys. B 23, 4377-4400.

Karwasz, G. P., Brusa, R. S., and Zecca, A. (2001a). One century of experiments on electronatom and molecule scattering: a critical review of integral cross sections. II. Polyatomic molecules. Riv. Nuovo Cim. 24(1), 1-118.

Karwasz, G. P., Brusa, R. S., and Zecca, A. (2001b). One century of experiments on electronatom and molecule scattering: a critical review of integral cross-sections. III. Hydrocarbons and halides. Riv. Nuovo Cim. 24(4), 1-101.

Kasperovich, V., Tikhonov, G., Wong, K., and Kresin, V. V. (2000a). Negative-ion formation in collisions of low-energy electrons with neutral sodium clusters. Phys. Rev. A 62, 063201(1-6).

Kasperovich, V., Wong, K., Tikhonov, G., and Kresin, V. V. (2000b). Electron capture by the image charge of a metal nanoparticle. Phys. Rev. Lett. 85, 2729-2732.

Kasperovich, V., Tikhonov, G., and Kresin, V. V. (2001). Low-energy electron capture by free $\mathrm{C}_{60}$ and the importance of polarization interaction. Chem. Phys. Lett. 337, 55-60.

Kau, R., Petrov, I. D., Sukhorukov, V. L., and Hotop, H. (1998). Experimental and theoretical cross sections for photoionization of metastable Ar* and $\mathrm{Kr}^{*}$ atoms near threshold. J. Phys. B. 31, 1011-1027.

Kazansky, A. K. (1978). Model of the passing of the s term through the parabolic boundary of the continuum. Theor. Math. Phys. 36, 825-832.

Kazansky, A. K. (1982). Antibonding intermediate state in the theory of vibrational excitation of diatomic molecules by slow electrons. Sov. Phys. JETP 55, 824-827.

Kazansky, A. K. (1995). A model study of dissociative attachment of slow electrons to the carbon dioxide molecule. J. Phys. B 28, 3987-4004.

Kazansky, A. K., and Fabrikant, I. I. (1984). Scattering of slow electrons by molecules. Sov. Phys. Usp. 27, 607-630.

Keil, M., Kolling, T., Bergmann, K. and Meyer, W. (1999). Dissociative attachment of lowenergy electrons to vibrationally excited $\mathrm{Na}_{2}$ molecules using a photoelectron source. Eur. Phys. J. D 7, 55-64.

Kennerly, R. E., Van Brunt, R. J., and Gallagher, A. C. (1981). High-resolution measurement of the helium $1 \mathrm{~s} 2 \mathrm{~s}^{2}{ }^{2} \mathrm{~S}$ resonance profile. Phys. Rev. A 23, 2430-2442.

Kimura, M., Sueoka, O., Hamada, A., and Itikawa, Y. (2000). A comparative study of electronand positron-polyatomic molecule scattering. Adv. Chem. Phys. 111, 537-622.

Kitajima, M., Watanabe, S., Tanaka, H., Takekawa, M., Kimura, M., and Itikawa, Y. (2000). Strong mode dependence of the 3.8-eV resonance in $\mathrm{CO}_{2}$ vibrational excitation by electron impact. Phys. Rev. A 61, 060701 (R1-R4).

Klar, D., Ruf, M.-W., and Hotop, H. (1992a). Attachment of electrons to molecules at submillielectronvolt resolution. Chem. Phys. Lett. 189, 448-454.

Klar, D., Ruf, M.-W., and Hotop, H. (1992b). Attachment of electrons to molecules at meV resolution. Aust. J. Phys. 45, 263-291.

Klar, D., Ruf, M.-W., and Hotop, H. (1994a). A tunable laser-atomic beam photoelectron source with sub-milli-electron-volt resolution: design, operation and application to threshold electron attachment studies. Meas. Sci. Technol. 5, 1248-1261.

Klar, D., Mirbach, B., Korsch, H. J., Ruf, M.-W., and Hotop, H. (1994b). Comparison of rate coefficients for Rydberg electron and free electron attachment. Z. Phys. D 31, 235-244.

Klar, D., Ruf, M.-W., and Hotop, H. (2001a). Dissociative electron attachment to $\mathrm{CCl}_{4}$ molecules at low electron energies with meV resolution. Int. J. Mass Spectrom. 205, 93-110. 
Klar, D., Ruf, M.-W., Fabrikant, I. I., and Hotop, H. (2001b). Dissociative electron attachment to dipolar molecules at low energies with meV resolution: $\mathrm{CFCl}_{3}, 1,1,1-\mathrm{C}_{2} \mathrm{Cl}_{3} \mathrm{~F}_{3}$, and $\mathrm{HI}$. J. Phys. B 34, 3855-3878.

Klots, C. E. (1976). Rate constants for unimolecular decomposition at threshold. Chem. Phys. Lett. 38, 61-64.

Klots, C. E., and Compton, R. N. (1977). Electron attachment to carbon dioxide clusters in a supersonic beam. J. Chem. Phys. 67, 1779-1780.

Klots, C. E., and Compton, R. N. (1978). Electron attachment to van der Waals polymers of carbon dioxide and nitrous oxide. J. Chem. Phys. 69, 1636-1643.

Klots, C. E., and Compton, R. N. (1980). Self-scavenging of electrons in van der Waals molecules of methyl iodide. Chem. Phys. Lett. 73, 589-591.

Klots, C. E., and Compton, R. N. (1996). Evidence for thermionic emission from small aggregates. Surf. Rev. Lett. 3, 535-540.

Knapp, M., Kreisle, D., Echt, O., Sattler, K., and Recknagel, E. (1985). Size distributions of negatively and positively charged clusters: $\mathrm{CO}_{2}$ and $\mathrm{N}_{2} \mathrm{O}$. Surf. Science 156, 313-320.

Knapp, M., Echt, O., Kreisle, D., Märk, T. D., and Recknagel, E. (1986a). Formation of longlived $\mathrm{CO}_{2}^{-}, \mathrm{N}_{2} \mathrm{O}^{-}$, and their dimer anions, by electron attachment to van der Waals clusters. Chem. Phys. Lett. 126, 225- 231.

Knapp, M., Echt, O., Kreisle, D., and Recknagel, E. (1986b). Trapping of low energy electrons at preexisting, cold water clusters. J. Chem. Phys. 85, 636-637.

Knapp, M., Echt, O., Kreisle, D., and Recknagel, E. (1987). Electron attachment to water clusters under collision-free conditions. J. Phys. Chem. 91, 2601-2607.

Knoth, G., Gote, M., Rädle, M., Jung, K., and Ehrhardt, H. (1989a). Nuclear-excited feshbach resonances in the electron scattering from hydrogen halides. Phys. Rev. Lett. 62, 1735-1737.

Knoth, G., Rädle, M., Gote, M., Ehrhardt, H., and Jung, K. (1989b). Near-threshold electron impact rovibrational excitation of $\mathrm{HCl}$ and HF. J. Phys. B 22, 299-326.

Kochem, K. H., Sohn, W., Hebel, N., Jung, K., and Ehrhardt, H. (1985). Elastic electron scattering and vibrational excitation of $\mathrm{CO}_{2}$ in the threshold energy region. J. Phys. B $\mathbf{1 8}$, 4455-4467.

Kolorenc, P., Čizek, M., Horáček, J., Mil'nikov, G., and Nakamura, H. (2002). Study of dissociative electron attachment to HI molecule by using $R$-matrix representation for Green's function. Physica Scripta 65, 328-335.

Kondow, T., and Mitsuke, K. (1985). Formation of negative cluster ions of $\mathrm{CO}_{2}$, OCS, and $\mathrm{CS}_{2}$ produced by electron transfer from high-Rydberg rare gas atoms. J. Chem. Phys. 83, 212-213.

Kondow, T. (1987). Ionization of clusters in collision with high-Rydberg rare gas atoms. J. Phys. Chem. 91, 1307-1316.

Kovalchuk, E. V., Dekorsy, D., Lvovsky, A. I., Braxmaier, C., Mlynek, J., Peters, A., and Schiller, S. (2001). High-resolution Doppler-free molecular spectroscopy with a continuouswave optical parametric oscillator. Opt. Lett. 26, 1430-1432.

Kraft, T., Ruf, M.-W., and Hotop, H. (1989). Formation of negatively-charged cluster ions in thermal energy collisions with state-selected Rydberg atoms. Z. Phys. D 14, 179-185.

Kraft, T., Ruf, M.-W., and Hotop, H. (1990). Strong dependence of negative cluster ion spectra on principal quantum number $n$ in collisions of state-selected $\operatorname{Ar}^{* *}(n \mathrm{~d})$ Rydberg atoms with $\mathrm{N}_{2} \mathrm{O}$ clusters. Z. Phys. D 17, 37-434.

Kraft, T., Ruf, M.-W., and Hotop, H. (1991). Effective absolute rate constant for the formation of negatively-charged $\mathrm{CO}_{2}$ cluster ions by electron transfer from state-selected Rydberg atoms. Z. Phys. D 18, 403-407. 
Kreil, J., Ruf, M.-W., Hotop, H., Ettischer, I., and Buck, U. (1998). Threshold electron attachment and electron impact ionization involving oxygen dimers. Chem. Phys. 239, 459-473.

Krishnakumar, E., and Srivastava, S. K. (1990). Dissociative attachment of electrons to $\mathrm{N}_{2} \mathrm{O}$. Phys. Rev. A 41, 2445-2452.

Külz, M., Keil, M., Kortyna, M., Schellhaaß, B., Hauck, J., Bergmann, K., and Meyer, W. and Weyh, D. (1996). Dissociative attachment of low-energy electrons to state-selected diatomic molecules. Phys. Rev. A 53, 3324-3334.

Kurepa, M. V., and Belić, D. S. (1978). Electron-chlorine molecule total ionisation and electron attachment cross sections. J. Phys. B 11, 3719-3729.

Land, J. E., and Raith, W. (1974). High-resolution measurement of resonances in $e-\mathrm{O}_{2}$ scattering by electron time-of-flight spectroscopy. Phys. Rev. A 9, 1592-1602.

Landau, L. D., and Lifshitz, E. M. (1977). "Quantum Mechanics, Non-relativistic Theory," 3rd Edn., Pergamon, Oxford.

Lane, A. M., and Thomas, R. G. (1958). R-matrix theory of nuclear reactions. Rev. Mod. Phys. 30, 257-353.

Lane, N. F. (1980). The theory of electron-molecule collisions, Rev. Mod. Phys. 52, 29-119.

Langevin, P. (1905). Une formule fondamentale de théorie cinétique. Ann. chim. et phys. 5, 245-288.

Larrichia, G., and Wilkin, C. (1997). Semiempirical approach to positron annihilation in molecules. Phys. Rev. Lett. 79, 2241-2244.

Leber, E., Fabrikant, I. I., Weber, J. M., Ruf, M.-W., and Hotop, H. (2000a). Resonance and threshold phenomena in electron attachment to molecules and clusters. In: M. Larsson, J. B. A. Mitchell, and I. F. Schneider, Eds. "Dissociative Recombination: Theory, experiment and applications IV," pp. 69-76, World Scientific, Singapore.

Leber, E., Barsotti, S., Fabrikant, I. I., Weber, J. M., Ruf, M.-W., and Hotop, H. (2000b). Vibrational feshbach resonances in electron attachment to carbon dioxide clusters. Eur. Phys. J. D 12, 125-131.

Leber, E., Barsotti, S., Bömmels, J., Weber, J. M., Fabrikant, I. I., Ruf, M.-W., and Hotop, H. (2000c). Vibrational Feshbach resonances in electron attachment to nitrous oxide clusters: decay into heterogeneous and homogeneous cluster anions. Chem. Phys. Lett. 325, 345-353.

LeGarrec, J.-L., Steinhurst, D. A., and Smith, M. A. (2001). Measurement of the autodetachment lifetime of $\mathrm{SF}_{6}^{-}$as a function of electron energy in a free jet expansion. J. Chem. Phys. 114, 8831-8835.

Lehr, L., and Miller, W. H. (1996). A classical approach to dissociative electron attachment DA: application to temperature effects in the DA cross section of $\mathrm{CF}_{3} \mathrm{Cl}$. Chem. Phys. Lett. 250, 515-522.

Lehr, L., Manz, J., and Miller, W. H. (1997). A classical approach to resonant low-energy electron scattering of molecules: application to the $\mathrm{a}_{1}$-shape resonance of $\mathrm{CF}_{3} \mathrm{Cl}$. Chem. Phys. 214, 301-312.

Leininger, T., and Gadea, F. X. (2000). Ab initio calculations for electron attachment to $\mathrm{Cl}_{2}$. J. Phys. B 33, 735-744.

Lezius, M., Scheier, P., and Märk, T. D. (1993). Free electron attachment to $\mathrm{C}_{60}$ and $\mathrm{C}_{70}$. Chem. Phys. Lett. 203, 232-236.

Lide, D. R. (Ed.) (1995). "CRC Handbook of Chemistry and Physics," 76th Edn. Boca Raton, FL: Chemical Rubber Company.

Ling, X., Lindsay, B. G., Smith, K. A., and Dunning, F. B. (1992). Rydberg-atom collisions with $\mathrm{SF}_{6}$ and $\mathrm{CCl}_{4}$ at very high $n$. Phys. Rev. A 45, 242-246.

Lucchese, R. R., Gianturco, F. A., and Sanna, N. (1999). Low-energy electron scattering from $\mathrm{C}_{60}$ molecules. Chem. Phys. Lett. 305, 413-418. 
Lunt, S. L., Field, D., Ziesel, J.-P., Jones, N. C., and Gulley, R. J. (2001). Very low energy electron scattering in nitromethane, nitroethane, and nitrobenzene. Int. J. Mass Spectrom. 205, 197-208.

Maeyama, T., Oikawa, T., Tsumura, T., and Mikami, N. (1998). Photodestruction spectroscopy of carbon disulfide cluster anions $\left(\mathrm{CS}_{2}\right)_{n}^{-}, n=1-4$ : evidence for the dimer core structure and competitive reactions of the dimer anion. J. Chem. Phys. 108, 1368-1376.

Maillet, J.-B., Boutin, A., Buttefey, S., Calvo, F., Fuchs, A. H. (1998). From molecular clusters to bulk matter. I. Structure and thermodynamics of small $\mathrm{CO}_{2}, \mathrm{~N}_{2}$, and $\mathrm{SF}_{6}$ clusters. J. Chem. Phys. 109, 329-337.

Märk, T. D. (1991). Free electron attachment to van der Waals clusters. Int. J. Mass Spectrom. Ion Processes 107, 143-163.

Märk, T. D., Leiter, K., Ritter, W., and Stamatovic, A. (1985). Low-energy-electron attachment to oxygen clusters produced by nozzle expansion. Phys. Rev. Lett. 55, 2559-2562.

Märk, T. D., Leiter, K., Ritter, and W., Stamatovic, A. (1986). Electron attachment to oxygen clusters at low electron energies. Formation and stability of $\left(\mathrm{O}_{2}\right)_{\mathrm{n}}^{-}$and $\left(\mathrm{O}_{2}\right)_{\mathrm{n}} \mathrm{O}^{-}$. Int. J. Mass Spectrom. Ion Proc. 74, 265-279.

Massey, H. S. W. (1976). "Negative Ions," 3rd Edn. Cambridge Univ. Press, Cambridge.

Matejčik, Š., Märk, T. D., Španel, P., Smith, D., Jaffke, T., and Illenberger, E. (1995). Formation and decay of $\mathrm{C}_{60}^{-}$following free electron capture by $\mathrm{C}_{60} . J$. Chem. Phys. 102, 2516-2521.

Matejcik, S., Kiendler, A., Stampfli, P., Stamatovic, A., and Märk, T. D. (1996). Vibrationally resolved electron attachment to oxygen clusters. Phys. Rev. Lett. 77, 3771-3774.

Matejcik, S., Stampfli, P., Stamatovic, A., Scheier, P., and Märk, T. D. (1999). Electron attachment to oxygen clusters studied with high energy resolution. J. Chem. Phys. 111, 3548-3558.

Matsuzawa, M. (1972). Reactions of highly excited atoms with molecules $\mathrm{A}^{* *}+\mathrm{BC} \rightarrow$ $\mathrm{A}^{+}+\mathrm{B}+\mathrm{C}^{-}$. J. Phys. Soc. Japan 32, 1108-1119.

Mazevet, S., Morrison, M. A., Morgan, L. A., and Nesbet, R. K. (2001). Virtual-state effects on elastic scattering and vibrational excitation of $\mathrm{CO}_{2}$ by electron impact. Phys. Rev. A 64, 040701(1-4).

McCorkle, D. L., Christodoulides, A. A., and Christophorou, L. G. (1984). Electron attachment to $\mathrm{Cl}_{2}$. Chem. Phys. Lett. 109, 276-279.

McCurdy, C. W., and Turner, J. L. (1983). Wave packet formulation of the boomerang model for resonant electron-molecule scattering. J. Chem. Phys. 78, 6773-6779.

Meyer, H.-D., Horáček, J., and Cederbaum, L. S. (1991). Schwinger and anomaly-free Kohn variational principles and a generalized Lanczos algorithm for nonsymmetric operators. Phys. Rev. A 43, 3587-3596.

Miller, D. R. (1988). Free jet sources. In: G. Scoles, Ed. "Atomic and molecular beam methods," Vol. 1, pp. 14-53, Oxford Univ. Press, New York.

Misaizu, F., Mitsuke, K., Kondow, T., and Kuchitsu, K. (1991). Formation of negative cluster ions from $\left(\mathrm{CO}_{2}\right)_{m}$ in collision with high-Rydberg atoms. J. Chem. Phys. 94, 243-249.

Mitchell, J. B. A. (1990). The dissociative recombination of molecular ions. Phys. Rep. 186, 215-248.

Mittleman, M. H., and von Holdt, R. E. (1965). Theory of low-energy-electron scattering by polar molecules. Phys. Rev. 140, A726-A729.

Modelli, A., Scagnolari, F., Distefano, G., Jones D., and Guerra, M. (1992). Electron attachment to the fluoro-, bromo-, and iodomethanes studied by means of electron transmission spectroscopy and $\mathrm{X} \alpha$ calculations. J. Chem. Phys. 96, 2061-2070.

Morgan, L. A. (1998). Virtual states and resonances in electron scattering by $\mathrm{CO}_{2}$. Phys. Rev. Lett. 80, 1873-1875. 
Morrison, M. A. (1982). Interpretation of the near-threshold behavior of cross sections for $e-\mathrm{CO}_{2}$ scattering. Phys. Rev. A 25, 1445-1449.

Morrison, M. A. (1988). Near-threshold electron-molecule scattering. Adv. At. Mol. Phys. 24, 51-156.

Murphy, T. J., and Surko, C. M. (1991). Annihilation of positrons on organic molecules. Phys. Rev. Lett. 67, 2954-2957.

Oakes, J. M., and Ellison, G. B. (1986). Photoelectron spectroscopy of radical anions. Tetrahedron 42, 6263-6267.

O'Malley, T. F. (1965). Effect of long-range final-state forces on the negative-ion photodetachment cross section near threshold. Phys. Rev. 137, A1668-A1672.

O’Malley, T. F. (1966). Theory of dissociative attachment. Phys. Rev. 150, 14-29.

O'Malley, T. F. (1967). Calculation of dissociative attachment in hot $\mathrm{O}_{2}$. Phys. Rev. 155, 59-63.

O'Malley, T. F. (1981). Rydberg levels and structure in dissociative recombination cross sections. J. Phys. B 14, 1229-1238.

O'Malley, T. F., Rosenberg, L., and Spruch, L. (1962). Low-energy scattering of a charged particle by a neutral polarizable system. Phys. Rev. 125, 1300-1310.

Orient, O. J., Chutjian, A., Crompton, R. W., and Cheung, B. (1989). Comparison of experimental and calculated attachment rate constants for $\mathrm{CFCl}_{3}$ and $\mathrm{CCl}_{4}$ in the temperature range 294-500 K. Phys. Rev. A 39, 4494-4501.

Osterwalder, A., and Merkt, F. (1999). Using high rydberg states as electric field sensors. Phys. Rev. Lett. 82, 1831-1834.

Pastuszka, S., Hoppe, M., Kratzmann, D., Schwalm, D., Wolf, A., Jaroshevich, A. S., Kosolobov, S. N., Orlov, D. A., and Terekhov, A. S. (2000). Preparation and performance of transmission-mode GaAs photocathodes as sources for cold dc electron beams. J. Appl. Phys. 88, 6788-6800.

Paul, D. A. L., and Saint-Pierre, L. (1963). Rapid annihilations of positrons in polyatomic gases. Phys. Rev. Lett. 11, 493-496.

Pearl, D. M., and Burrow, P. D. (1993). Thermal decomposition and the apparent dissociative attachment cross section of heated methyl-, ethyl- and $t$-butyl-chloride. Chem. Phys. Lett. 206, 483-487.

Pearl, D. M., Burrow, P. D., Fabrikant, I. I., and Gallup, G. A. (1995). Dissociative attachment in hot $\mathrm{CH}_{3} \mathrm{Cl}$ : experiment and theory. J. Chem. Phys. 102, 2737-2743.

Petrov, I. D., Sukhorukov, V. L., and Hotop, H. (1999). The influence of core polarization on photoionization of alkali and metastable rare gas atoms near threshold. J. Phys. B 32, 973-986.

Petrov, I. D., Sukhorukov, V. L., Leber, E., and Hotop, H. (2000). Near threshold photoionization of excited alkali atoms $\mathrm{Ak}(\mathrm{np})(\mathrm{Ak}=\mathrm{Na}, \mathrm{K}, \mathrm{Rb}, \mathrm{Cs} ; \mathrm{n}=3-6)$. Eur. Phys. J. D 10,53-65.

Petrović, Z. Lj., and Crompton, R. W. (1985). Thermal-electron attachment to $\mathrm{SF}_{6}$ at room temperature and 500 K. J. Phys. B 18, 2777-2791.

Petrović, Z. Lj., and Crompton, R. W. (1987). Attachment and diffusion of thermal electrons in methyl bromide. J. Phys. B 20, 5557-5565.

Petrović, Z. Lj., Wang, W. C., and Lee, L. C. (1989). Dissociative electron attachment to some chlorine-containing molecules. J. Chem. Phys. 90, 3145-3152.

Popple, R. A., Finch, C. D., Smith, K. A., and Dunning, F. B. (1996). Dissociative electron attachment to $\mathrm{CCl}_{4}$ : lifetime of the $\mathrm{CCl}_{4}{ }^{-*}$ intermediate. J. Chem. Phys. 104, 8485-8489.

Rädle, M., Knoth, G., Jung, K., and Ehrhardt, H. (1989). Rotational and rovibrational excitation of $\mathrm{HCl}$ and $\mathrm{HF}$ by low-energy electron impact. J. Phys. B 22, 1455-1476.

Randell, J., Field, D., Lunt, S. L., Mrotzek, G., and Ziesel, J. P. (1992). Low-energy electron scattering by $\mathrm{SF}_{6}$. J. Phys. B 25, 2899-2909. 
Rauth, T., Foltin, M., and Märk, T. D. (1992). Autoscavenging of electrons in mixed van der Waals clusters: a new approach to the spectroscopy of cluster anions. J. Phys. Chem. 96, $1528-1530$.

Read, F. H., and Channing, J. M. (1996). Production and optical properties of an unscreened but localized magnetic field. Rev. Sci. Instrum. 67, 2372-2377.

Rescigno, T. N., Byrum, D. A., Isaacs, W. A., and McCurdy, C. W. (1999). Theoretical studies of low-energy electron- $\mathrm{CO}_{2}$ scattering: total, elastic, and differential cross sections. Phys. Rev. A 60, 2186-2193.

Rescigno, T. N., Isaacs, W. A., Orel, A. E., Meyer, H.-D., and McCurdy, C. W. (2002). Theoretical study of resonant vibrational excitation of $\mathrm{CO}_{2}$ by electron impact. Phys. Rev. A 65, $032716(1-13)$.

Rohr, K. (1977). Threshold resonances in vibrational excitation of $\mathrm{HBr}$ molecules by electron impact. J. Phys. B 10, L399-L402.

Rohr, K. (1978). Interaction mechanismus and cross sections for the scattering of low-energy electrons from HBr. J. Phys. B 11, 1849-1860.

Rohr, K., and Linder, F. (1975). Vibrational excitation in e-HCl collisions at low energies. J. Phys. B 8, L200-L204.

Rohr, K., and Linder, F. (1976). Vibrational excitation of polar molecules by electron impact. I. Threshold resonance in HF and HCl. J. Phys. B 9, 2521-2537.

Rosmus, P., and Hochlaf, M. (2002). Private communication.

Ross, M. H., and Shaw, G. L. (1961). Multichannel effective range theory. Ann. Phys. 13, $147-186$

Ruf, M.-W., Barsotti, S., Braun, M., Hotop, H., and Fabrikant, I. I. (2003). Attachment and vibrational excitation in low-energy electron collisions with chlorine molecules. $J$. Phys. B (submitted).

Ryzhikh, G. G., and Mitroy, J. (1997). Positronic Lithium, an electronically stable Li- $e^{+}$ground state. Phys. Rev. Lett. 79, 4124-4126.

Sadeghpour, H. R., Bohn, J. L., Cavagnero, M. J., Esry, B. D., Fabrikant, I. I., Macek, J. H., and Rau, A. R. P. (2000). Collisions near threshold in atomic and molecules physics. J. Phys. $B$ 33, R93-R140.

Sandner, W., Gallagher, T. F., Safinya, K. A., and Gounand, F. (1981). Photoionization of potassium in the vicinity of the minimum in the cross section. Phys. Rev. A 23, 2732-2735.

Saeki, M., Tsukuda, T., and Nagata, T. (2001). Ab initio study of $\left(\mathrm{CO}_{2}\right)_{\mathrm{n}}^{-}$: Structures and stabilities of isomers. Chem. Phys. Lett. 340, 376-384.

Sanov, A., Lineberger, W. C., and Jordan, K. D. (1998). Electronic structure of $\left(\mathrm{CS}_{2}\right)_{2}^{-}$. J. Phys. Chem. A 102, 2509-2511.

Sarpal, B. K., Pfingst, K., Nestmann, B. M., and Peyerimhoff, S. D. (1996). Study of electron scattering by $\mathrm{N}_{2} \mathrm{O}$ using the polyatomic $R$-matrix method. J. Phys. B 29, 857-873; J. Phys. Chem. A 102, 1877(E).

Schafer, O., and Allan, M. (1991). Measurement of near-threshold vibrational excitation of $\mathrm{HCl}$ by electron impact. J. Phys. B 24, 3069-3076.

Schneider, B. I., LeDourneuf, M., and Burke, P. G. (1979). Theory of vibrational excitation and dissociative attachment: an $R$-matrix approach. J. Phys. B 12, L365-L369.

Schohl, S., Klar, D., Cherepkov, N. A., Petrov, I. D., Ueda, K., Baier, S., Kau, R., and Hotop, H. (1997). Photoionization of polarized $\mathrm{Ar}^{*}(4 \mathrm{p}, J=3)$ atoms near threshold. J. Phys. B $\mathbf{3 0}$, 609-631.

Schramm, A., Fabrikant, I. I., Weber, J. M., Leber, E., Ruf, M.-W., and Hotop, H. (1999). Vibrational resonance and threshold effects in inelastic electron collisions with methyl iodide molecules. J. Phys. B 32, 2153-2171. 
Schramm, A., Ruf, M.-W., Stano, M., Matejcik, S., Fabrikant, I. I., and Hotop, H. (2002). High-resolution study of dissociative electron attachment to dipolar molecules at low energies: $\mathrm{CH}_{2} \mathrm{Br}_{2}$ and $\mathrm{CCl}_{3} \mathrm{Br}$. J. Phys. B 35, 4179-4202.

Schramm, A., Weber, J. M., Kreil, J., Klar, D., Ruf, M.-W., and Hotop, H. (1998). Laser photoelectron attachment to molecules in a skimmed supersonic beam: diagnostics of weak electric fields and attachment cross sections down to $20 \mu \mathrm{eV}$. Phys. Rev. Lett. 81, 778-781.

Schulz, G. J. (1973a). Resonances in electron impact on atoms. Rev. Mod. Phys. 45, 378-422.

Schulz, G. J. (1973b). Resonances in electron impact on diatomic molecules. Rev. Mod. Phys. 45, 423-486.

Senn, G., Skalny, J. D., Stamatovic, A., Mason, N. J., Scheier, P., and Märk, T. D. (1999). Low energy dissociative electron attachment to ozone. Phys. Rev. Lett. 82, 5028-5031.

Sentürk, Ş., Connerade, J. P., Burgess, D. D., and Mason, N. J. (2000). Enhanced electron capture by metallic clusters. J. Phys. B 33, 2763-2774.

Sergenton, A.-Ch., and Allan, M. (2000). Excitation of vibrational levels of HI up to v $=8$ by electron impact. Chem. Phys. Lett. 319, 179-183.

Sergenton, A.-Ch., Jungo, L., and Allan, M. (2000). Excitation of vibrational levels of HF up to $\mathrm{v}=4$ by electron impact. Phys. Rev. A 61,062702(1-6).

Shi, X., Chan, V. K., Gallup, G. A., and Burrow, P. D. (1996). Low energy electron scattering from $\mathrm{CH}_{3} \mathrm{Cl}$. J. Chem. Phys. 104, 1855-1863.

Shi, X. (1992). Ph.D. Thesis, Univ. of Nebraska, Lincoln, NE, USA.

Shimamori, H., and Nakatani, Y. (1988). Rate constants for thermal electron attachment to $\mathrm{CF}_{3} \mathrm{I}, \mathrm{CH}_{3} \mathrm{I}, \mathrm{C}_{2} \mathrm{H}_{5} \mathrm{I}, 1-\mathrm{C}_{3} \mathrm{H}_{7} \mathrm{I}$ and $2-\mathrm{C}_{3} \mathrm{H}_{7} \mathrm{I}$ determined between 250 and $350 \mathrm{~K}$. Chem. Phys. Lett. 150, 109-112.

Shimamori, H., Tatsumi, Y., Ogawa, Y., and Sunagawa, T. (1992a). Electron-energy dependence of electron attachment to $\mathrm{c}-\mathrm{C}_{7} \mathrm{~F}_{14}, \mathrm{CH}_{3} \mathrm{I}$ and $\mathrm{CH}_{2} \mathrm{Br}_{2}$ studied by the pulseradiolysis microwave-cavity technique combined with microwave heating. Chem. Phys. Lett. 194, 223-229.

Shimamori, H., Tatsumi, Y., Ogawa, Y., and Sunagawa, T. (1992b). Low-energy electron attachment to molecules studied by pulse-radiolysis microwave-cavity technique combined with microwave heating. J. Chem. Phys. 97, 6335-6347.

Shimamura, I., and Takayanagi, K. (Eds.) (1984). "Electron-Molecule Collisions," Plenum Press, New York.

Skalický, T., Chollet, C., Pasquier, N., and Allan, M. (2002). Properties of the $\pi^{*}$ and $\sigma^{*}$ states of chlorobenzene anion determined by electron impact spectroscopy. Phys. Chem. Chem. Phys. 4, 3583-3590.

Smith, D., and Španel, P. (1994). Studies of electron attachment at thermal energies using the flowing Afterglow-Langmuir probe technique. Adv. At. Mol. Opt. Phys. 32, 307-343.

Smith, D., and Spanel, P. (1996). Electron attachment to $\mathrm{C}_{60}$ and $\mathrm{C}_{70}$ in the gas phase. J. Phys. $B$ 29, 5199-5212.

Smith, D., Španel, P., and Märk, T. D. (1993). Electron attachment to $C_{60}$ at low energies. Chem. Phys. Lett. 213, 202-206.

Smith, J. R., Kim, J. B., and Lineberger, W. C. (1997). High-resolution threshold photodetachment spectroscopy of $\mathrm{OH}^{-}$. Phys. Rev. A 55, 2036-2043.

Sohn, W., Kochem, K.-H., Scheuerlein, K. M., Jung, K., and Ehrhardt, H. (1987). Low-energy electron impact spectroscopy of OCS and $\mathrm{CS}_{2}$. J. Phys. B 20, 3217-3236.

Sommerfeld, T. (2002). Coupling between dipole-bound and valence states: the nitromethane anion. Phys. Chem. Chem. Phys. 4, 2511-2516.

Sommerfeld, T. (2003). A fresh look at the ${ }^{2} \mathrm{~A}_{1} \mathrm{CO}_{2}^{-}$potential energy surface. J. Phys. B 36, L127-L133. 
Španel, P., Matejcik, S., and Smith, D. (1995). The varying influences of gas and electron temperatures on the rates of electron attachment to some selected molecules. J. Phys. B $\mathbf{2 8}$, 2941-2957.

Speck, T., Mostefaoui, T., Rebrion-Rowe, C., Mitchell, J. B. A., and Rowe, B. R. (2000). Low temperature electron attachment to $\mathrm{CH}_{3}$ I. J. Phys. B 33, 3575-3582.

Spence, D., and Schulz, G. J. (1973). Temperature dependence of electron attachment at low energies for polyatomic molecules. J. Chem. Phys. 58, 1800-1803.

Stamatovic, A. (1988). Electron attachment to van der Waals clusters: the zero energy resonance. In: H. B. Gilbody, W. R. Newell, F. H. Read, A. C. H. Smith, Eds. "Electronic and Atomic Collisions," pp. 729-735, North Holland, Amsterdam.

Stamatovic, A., Stephan, K., and Märk, T. D. (1985a). Electron attachment and electron ionisation of van der Waals clusters of carbon dioxide. Int. J. Mass Spectrom. Ion Proc. 63, 37-47.

Stamatovic, A., Leiter, K., Ritter, W., Stephan, K., and Märk, T. D. (1985b). Electron attachment to carbon dioxide clusters at very low electron energies. J. Chem. Phys. 83, 2942-2946.

Stampfli, P. (1995). Theory for the electron affinity of clusters of rare gas atoms and polar molecules. Phys. Rep. 255, 1-77.

Stebbings, R. F., and Dunning, F. B. (Eds.) (1983). "Rydberg States of Atoms and Molecules," Cambridge Univ. Press, Cambridge.

Stepanović, M., Pariat, Y., and Allan, M. (1999). Dissociative electron attachment in cyclopentanone, $\gamma$-butyrolactone, ethylene carbonate, and ethylene carbonate- $\mathrm{d}_{4}$ : role of dipole-bound resonances. J. Chem. Phys. 110, 11376-11382.

Suess, L., Parthasarathy, R., and Dunning, F. B. (2002). Nondissociative low-energy electron attachment to $\mathrm{SF}_{6}, \mathrm{C}_{6} \mathrm{~F}_{6}, \mathrm{C}_{10} \mathrm{~F}_{8}$, and $\mathrm{c}_{-} \mathrm{C}_{7} \mathrm{~F}_{14}$ : negative ion lifetimes. J. Chem. Phys. 117, $11222-11227$.

Surber, E., Ananthavel, S. P., and Sanov, A. (2002). Nonexistent electron affinity of OCS and the stabilization of carbonyl sulfide anions by gas phase hydration. J. Chem. Phys. 116, $1920-1929$.

Surko, C. M., Passner, A., Leventhal, M., and Wysocki, F. J. (1988). Bound states of positrons and large molecules. Phys. Rev. Lett. 61, 1831-1834.

Szmytkowski, C., Karwacz, G., and Maciag, K. (1984). Absolute total electron-scattering cross sections of $\mathrm{N}_{2} \mathrm{O}$ and OCS in the low-energy region. Chem. Phys. Lett. 107, 481-484.

Tachikawa, M., Shimamura, I., Buenker, R. J., and Kimura, M. (2001). In: F. Gianturco and C. Surko, Eds. "New Directions in Antimatter Chemistry and Physics," p. 437, Kluwer Academic.

Tam, W.-C., and Wong, S. F. (1978). Dissociative attachment of halogen molecules by $0-8 \mathrm{eV}$ electrons. J. Chem. Phys. 68, 5626-5630.

Tanaka, H., and Sueoka, O. (2001). Mechanisms of electron transport in electrical discharges and electron collision cross sections. Adv. At. Mol. Opt. Phys. 44, 1-32.

Teillet-Billy, D., and Gauyacq, J. P. (1984). Dissociative attachment in $\mathrm{e}^{-}-\mathrm{HCl}, \mathrm{DCl}$ collisions. J. Phys. B 17, 4041-4058.

Tennysson, J., and Morgan, L. (1999). Electron collisions with polyatomic molecules using the $R$-matrix method. Phil. Trans. R. Soc. Lond. A 357, 1161-1173.

Thoss, M., and Domcke, W. (1998). Theory of vibrational relaxation processes in resonant collisions of low-energy electrons with large molecules. J. Chem. Phys. 109, 6577-6595.

Thümmel, H. T., Nesbet, R. K., and Peyerimhoff, S. D. (1992). Near-threshold rotational excitation in electron-polar-molecule scattering. J. Phys. B 25, 4553-4579.

Thümmel, H. T., Nesbet, R. K., and Peyerimhoff, S. D. (1993). Near-threshold rovibrational excitation of HF by electron impact. J. Phys. B 26, 1233-1251. 
Torchet, G., Feraudy, M.-F., Boutin, A., and Fuchs, A. H. (1996). Structural transformation in $\left(\mathrm{CO}_{2}\right)_{N}$ clusters, $N<100$. J. Chem. Phys. 105, 3671-3678.

Tosatti, E., and Manini, N. (1994). Anomalous attachment of low-energy electrons to $\mathrm{C}_{60}$. Chem. Phys Lett. 223, 61-64.

Trajmar, S., and McConkey, J. W. (1994). Benchmark measurements of cross sections for electron collisions: analysis of scattered electrons. Adv. At. Mol. Opt. Phys. 33, 63-96.

Trajmar, S., Register, D. F., and Chutjian, A. (1983). Electron scattering by molecules. II. Experimental methods and data. Phys. Rep. 97, 219-356.

Travers, M. J., Cowles, D. C., and Ellison, G. B. (1989). Reinvestigation of the electron affinities of $\mathrm{O}_{2}$ and NO. Chem. Phys. Lett. 164, 449-455.

Tsukada, M., Shima, N., Tsuneyuki, S., Kageshima, H., and Kondow, T. (1987). Mechanism of electron attachment to van der Waals clusters: application to carbon dioxide clusters. J. Chem. Phys. 87, 3927-3933.

Tsukuda, T., Johnson, M. A., and Nagata, T. (1997a). Photoelectron spectroscopy of $\left(\mathrm{CO}_{2}\right)_{\mathrm{n}}^{-}$ revisited: core switching in the $2 \leq n \leq 16$ range. Chem. Phys. Lett. 268, 429-433.

Tsukuda, T., Hirose, T., and Nagata, T. (1997b). Negative-ion photoelectron spectroscopy of $\left(\mathrm{CS}_{2}\right)_{\mathrm{n}}^{-}$: coexistence of electronic isomers. Chem. Phys. Lett. 279, 179-184.

Turner, J. E. (1977). Minimum dipole moment required to bind an electron - molecular theorists rediscover phenomenon mentioned in Fermi-Teller paper 20 years earlier. Am. J. Phys. 45, 758-766.

Van Brunt, R. J., and Gallagher, A. (1978). Electron scattering with $1 \mathrm{meV}$ resolution. In: G. Watel (Ed.) "Electronic and Atomic Collisions," Proc. X. ICPEAC, Paris, 1977, pp. 129-142, North Holland Publ., Amsterdam.

Van Doren, J. M., Miller, T. M., Williams, S., and Viggiano, A. A. (2003). Thermal electron attachment to ozone, 296-550 K. Phys. Rev. Lett (submitted).

Van Reeth, P., and Humberston, J. W. (1998). The energy dependence of the annihilation rate in positron-atom scattering. J. Phys. B 31, L231-L238.

Vasil'ev, Y. V., Tuktarov, R. F., and Mazunov, V. A. (1997). Resonant electron capture mass spectra of fullerenes $\mathrm{C}_{60}$ and $\mathrm{C}_{70}$. Rapid Comm. Mass Spectrom. 11, 757-761.

Vasil'ev, Y. V., Abzalimov, R. R., Nasibullaev, S. K., Tuktarov, R. F., Pogulay, A. V., and Drewello, T. (2001). Low energy electron-molecule interactions of fullerene $\mathrm{C}_{60}$ and its hydro- and fluoro-derivatives. In: I. I. Fabrikant, G. A. Gallup, and P. D. Burrow, Eds. "Int. Symp. On Electron-Molecule Collisions and Swarms," Abstracts, pp. 57-58, Lincoln, NE, USA.

Vitanov, N. V., Fleischhauer, M., Shore, B. W., and Bergmann, K. (2001). Coherent manipulation of atoms and molecules by sequential laser pulses. Adv. At. Mol. Opt. Phys. 46, 55-190.

Vogt, E., and Wannier, G. H. (1954). Scattering of ions by polarization forces. Phys. Rev. 95, 1190-1198.

Walter, C. W., Zollars, B. G., Johnson, C. B., Smith, K. A., and Dunning, F. B. (1986). Formation of $\mathrm{O}_{2}^{-}$ions in $\mathrm{K}(n d)-\mathrm{O}_{2}$ collisions at intermediate $n$. Phys. Rev. A 34, 4431-4432.

Weber, J. M., Fabrikant, I. I., Leber, E., Ruf, M.-W., and Hotop, H. (2000). Effects of solvation on dissociative electron attachment to methyl iodide clusters. Eur. Phys. J. D 11, 247-256.

Weber, J. M., Leber, E., Ruf, M.-W., and Hotop, H. (1999a). Nuclear-excited Feshbach resonances in electron attachment to molecular clusters. Phys. Rev. Lett. 82, 516-519.

Weber, J. M., Leber, E., Ruf, M.-W., and Hotop, H. (1999b). Formation of small water cluster anions by attachment of very slow electrons at high resolution. Eur. Phys. J. D 7, 587-594.

Weber, J. M., Ruf, M.-W., and Hotop, H. (1996). Rydberg electron transfer to $\mathrm{C}_{60}$ and $\mathrm{C}_{70}$. Z. Phys. D 37, 351-357. 
Wigner, E. P. (1948). On the behavior of cross sections near thresholds. Phys. Rev. 73, 1002-1009.

Wigner, E. P., and Eisenbud, L. (1947). Higher angular momenta and long range interaction in resonance reactions. Phys. Rev. 72, 29-41.

Wilde, R. S., Gallup, G. A., and Fabrikant, I. I. (1999). Semiempirical $R$-matrix theory of low energy electron- $\mathrm{CF}_{3} \mathrm{Cl}$ inelastic scattering. J. Phys. B 32, 663-673.

Wilde, R. S., Gallup, G. A., and Fabrikant, I. I. (2000). Comparative studies of dissociative electron attachment to methyl halides. J. Phys. B 33, 5479-5492.

Winstead, C., and McKoy, V. (1998). Electron collisions with nitrous oxide. Phys. Rev. A 57, 3589-3597.

Winstead, C., and McKoy, V. (2000). Electron-molecule collisions in low-temperature plasmas. Adv. At. Mol. Opt. Phys. 43, 111-145.

Wong, C. F., and Light, J. C. (1984). Application of $R$-matrix theory to resonant reactive electron-molecule scattering: vibrational excitation and dissociative attachment of $\mathrm{N}_{2}$ and $\mathrm{F}_{2}$. Phys. Rev. A 30, 2264-2273.

Wong, C. F., and Light, J. C. (1986). Vibrational excitation and dissociative attachment of a triatomic molecule: $\mathrm{CO}_{2}$ in the collinear approximation. Phys. Rev. A 33, 954-967.

Xu, Y., Gallup, G. A., and Fabrikant, I. I. (2000). Dissociative electron attachment to vibrationally and rotationally excited $\mathrm{H}_{2}$ and HF molecules. Phys. Rev. A 61, 052705(1-7).

Zecca, A., Karwasz, G. P., and Brusa, R. S. (1996). One century of experiments on electronatom and molecule scattering: a critical review of integral cross-section. I. Atoms and diatomic molecules. Riv. Nuovo Cim. 19(3), 1-146.

Ziesel, J. P., Nenner, I., and Schulz, G. J. (1975a). Negative ion formation, vibrational excitation, and transmission spectroscopy in hydrogen halides. J. Chem. Phys. 63, 1943-1949.

Ziesel, J. P., Schulz, G. J., and Milhaud, J. (1975b). S $^{-}$formation by dissociative attachment in OCS and $\mathrm{CS}_{2}$. J. Chem. Phys. 62, 1936-1940.

Ziesel, J. P., Randell, J., Field, D., Lunt, S. L., Mrotzek, G. and Martin, P. (1993). Very low energy electron scattering by $\mathrm{O}_{2}$. J. Phys. B 26, 527-533.

Ziesel, J. P., Jones, N. C., Field, D., and Madsen, L. B. (2003). Reaction and scattering in cold electron collisions. Phys. Rev. Lett. 90, 083201 (1-4).

Zubek, M., Gulley, N., King, G. C., and Read, F. H. (1996). Measurements of elastic electron scattering in the backward hemisphere. J. Phys. B 29, L239-L244.

Zubek, M., Mielewska, B., Channing, J. M., King, G. C., and Read, F. H. (1999). A study of resonance structures in elastic electron scattering from helium, neon, argon, krypton, and xenon over the angular range from 100 to $180^{\circ}$. J. Phys. B 32, 1351-1363.

Zubek, M., Mielewska, B., Channing, J., King, G. C., and Read, F. H. (2000). Absolute differential cross sections for electron elastic scattering and vibrational excitation in nitrogen in the angular range from 120 to $180^{\circ}$. J. Phys. B 33, L527-L532. 